HELMUT MEDER

DIE INTERTEMPORALE

ALLOKATION

ERSCHÖPFBARER

NATURRESSOURCEN

BEI FEHLENDEN

ZUKUNFTSMÄRKTEN UND

INSTITUTIONALISIERTEN

MARKTSUBSTITUTEN 


\section{DIE INTERTEMPORALE ALLOKATION ERSCHÖPFBARER NATURRESSOURCEN BEI FEHLENDEN ZUKUNFTSMÄRKTEN UND INSTITUTIONALISIERTEN MARKTSUBSTITUTEN}

In der ökonomischen Theorie natürlicher Ressourcen erfolgt die Analyse der intertemporalen Allokation hauptsächlich unter vollkommener Information. Gibt man die Annahme eines vollständigen Marktsystems auf, Zukunfts- und Risikomärkte seien nicht vorhanden, dann fallen bestimmte Allokationsmechanismen aus und rufen bei den Wirtschaftssubjekten Reaktionen hervor, die für sie entstandenen Informationsmängel und Risiken zu reduzieren oder gar zu beseitigen. Dies kann geschehen, indem die Wirtschaftssubjekte die fehlenden Märkte durch geeignete Institutionen ersetzen. In dieser Arbeit erfolgt die Betrachtung solcher Institutionen. Es werden die intertemporalen Allokationswirkungen von langfristigen Lieferverträgen, von Terminkontrakten, von Optionskontrakten, von Konzessionen, von Verträgen zur Abbaumengenteilung und von Dienstleistungsverträgen analysiert.

Helmut Meder wurde 1950 in Landsberg am Lech geboren. Von 1974 bis 1979 Studium der Volkswirtschaftslehre, der Statistik und der Ökonometrie an der Universität Mannheim; von 1979 bis 1984 wissenschaftlicher Assistent am Lehrstuhl für Volkswirtschaftslehre und Außenwirtschaft an der Universität Mannheim. 
Die intertemporale Allokation erschöpfbarer Naturressourcen bei fehlenden Zukunftsmärkten und institutionalisierten Marktsubstituten 


\section{STAATLICHE ALLOKATIONSPOLITIK IM MARKTWIRTSCHAFTLICHEN SYSTEM}

Herausgegeben von

Klaus Conrad, Heinz König, Hans-Heinrich Nachtkamp, Rüdiger Pethig, Ulrich Schlieper, Horst Siebert, Eberhard Wille

\section{Band 11}

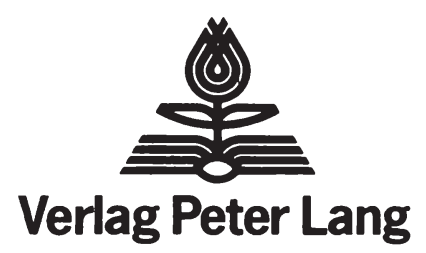

Frankfurt am Main - Bern - New York · Nancy 


\section{DIE INTERTEMPORALE ALLOKATION ERSCHOPFBARER NATURRESSOURCEN BEI FEHLENDEN ZUKUNFTSMÄRKTEN UND INSTITUTIONALISIERTEN MARKTSUBSTITUTEN}

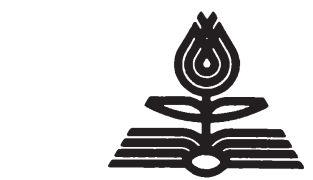

Verlag Peter Lang

Frankfurt am Main · Bern · New York · Nancy 
CIP-Kurztitelaufnahme der Deutschen Bibliothek

\section{Meder, Helmut:}

Die intertemporale Allokation erschöpfbarer

Naturressourcen bei fehlenden Zukunftsmärkten und

institutionalisierten Marktsubstituten / Helmut

Meder. - Frankfurt am Main ; Bern ; New York :

Lang, 1984.

(Staatliche Allokationspolitik im

marktwirtschaftlichen System ; Bd. 11)

ISBN 3-8204-8256-3

NE: GT

Open Access: The online version of this publication is published on www.peterlang.com and www.econstor.eu under the international Creative Commons License CC-BY 4.0. Learn more on how you can use and share this work: http://creativecommons.org/licenses/by/4.0.

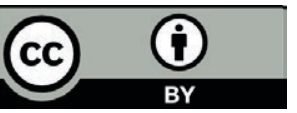

This book is available Open Access thanks to the kind support of ZBW - Leibniz-Informationszentrum Wirtschaft.

ISSN 0721-2860

ISBN 3-8204-8256-3

ISBN 978-3-631-75564-8 (eBook)

(C) Verlag Peter Lang $\mathrm{GmbH}$, Frankfurt am Main 1984

Alle Rechte vorbehalten.

Nachdruck oder Vervielfältigung, auch auszugsweise, in allen Formen wie Mikrofilm, Xerographie, Mikrofiche, Mikrocard, Otfset verboten.

Druck und Bindung: Weihert-Druck GmbH, Darmstadt 


\section{Vorwort}

Die Fakultät für Volkswirtschaftslehre und Statistik der Universität Mannheim hat diese Arbeit im Juni 1984 als Dissertation angenommen. Die Dissertationsgutachter waren Herr Prof. Dr. H. Siebert und Herr Prof. Dr. M. E. Streit. Beiden gilt mein Dank. Herrn Prof. Dr. H. Siebert habe ich nicht nur als Gutachter zu danken. In seiner Rolle als mein akademischer Lehrer habe ich ihm für zahlreiche Hilfestellungen und Ratschläge aufrichtig zu danken. Seine Anregungen waren mir willkommene Hilfen in den verschiedenen Phasen der Dissertation.

Wertvolle Hinweise erhielt ich ferner von meinen Kolleginnen Helga Gebauer, Anke Meyer, Sabine Toussaint und meinen Kollegen Dr. Ferdinand Dudenhöffer und Joachim Keck. Ihnen danke ich für die Anregungen, die sie mir in Seminaren und Einzeldiskussionen am Mannheimer Sonderforschungsbereich 5 "Staatliche Allokationspolitik im marktwirtschaftlichen System" gegeben haben.

Recht herzlich danken möchte ich auch Frau Anne-Lore Huber und meiner Frau Eva. Sie haben es sich Freizeit kosten lassen, um die Schreib- und Kommafehler auszumerzen. 
Helmut Meder - 978-3-631-75564-8

Downloaded from PubFactory at 01/11/2019 03:30:58AM

via free access 


\section{Einführung}

1.1. Einordnung und Problemstellung der Arbeit

1.2. Definition und Klassifikation des Allokationsobjektes Naturressource

2. Die intertemporale Allokation bei vollständigen Zukunftsmärkten

2.1. Das Polypol

2.1.1. Der gewinnorientierte Ansatz: Gewinnmaximierung der Eigentümer

2.1.2. Der umsatzorientierte Ansatz: Umsatzmaximierung durch Manager

2.1.3. Der nutzenorientierte Ansatz: Nutzenmaximierung durch Manager

2.2. Das Monopol

2.2.1. Ressourcenabbau bei antizipiertem Nachfrageverhalten

2.2.2. Ressourcenabbau und Preisdifferenzierung bei räumlich getrennten Märkten

2.3. Das Oligopol: Mengenkonkurrenz im homogenen Duopol

2.4. Ein zweistufiges Konzept zur theoretischen Interpretation der Funktionsweise von Rohstoff- und Reservenmarkt 
3. Die intertemporale Allokation bei fehlenden Zukunftsmärkten

3.1. Das Polypol 64

3.1.1. Der gewinnorientierte Ansatz 67

3.1.2. Der umsatzorientierte Ansatz 79

3.2. Das Monopol 83

3.2.1. Ressourcenabbau bei Antizipation eines risikobehafteten Nachfrageverlaufs

3.2.2. Ressourcenabbau und Preisdifferenzierung bei räumlich getrennten Märkten mit unterschiedlichem Risiko

3.3. Das Oligopol: Mengenkonkurrenz im homogenen Duopol

4. Institutionalisierte Substitute für fehlende Zukunftsmärkte

4.1. Substitutive Allokationsmechanismen für Rohstoffe

4.1.1. Kassamarkt, Terminmarkt, Terminkontraktmarkt, Optionsmarkt, langfristige Lieferverträge

4.1.2. Langfristige Lieferverträge auf dem Rohstoffmarkt

4.2. Substitutive Allokationsmechanismen für Reserven

4.2.1. Auktıon, diskretionäre Vergabe, Verhandlung 
4.2.2. Vertragsvereinbarungen auf dem Reservenmarkt

5. Die Allokationswirkungen institutionalisierter Marktsubstitute in Zweiperiodenmodellen des Ressourcenabbaus

5.1. Ressourcenabbau ohne Zukunftsmärkte

5.2. Ressourcenabbau mit "lang fristigen" Lieferverträgen

5.3. Ressourcenabbau mit Terminkontrakten

5.4. Ressourcenabbau mit Optionskontrakten

6. Die Allokationswirkungen von Vertragsvereinbarungen in Mehrperiodenmodellen des Ressourcenabbaus

6.1. Vertragsvereinbarungen auf dem Rohstoffmarkt

6.1.1. Bestimmungsfaktoren für den Abschluß von langfristigen Lieferverträgen unter Berücksichtigung des Nachfrageverhaltens eines rohstoffverarbeitenden Unternehmens

6.1.2. Das Abbauprofil bei konkurrenzmäßig organisiertem Kassa- und Vertragshandel

6.1.3. Das Abbauprofil bei monopolisiertem Vertragshandel

6.1.3.1. Polypolistischer Kassamarkt 
0.1.3.3. Vertráge mit unterschiedlicher Laufzeit

6.1.3.4. Verträge mit variierenden Liefermengen und Lieferpreisen

6.2. Vertragsvereinbarungen auf dem Reservenmarkt

6.2.1. Konzessionsmodelle

6.2.1.1. Deterministischer Reservenpreis bzw. mengenbezogene Forderabgabe

6.2.1.2. Indexierter Reservenpreis bzw. mengen- und wertbezogene Förderabgabe

6.2.2. Modelle zur Abbaumengenteilung

6.2.2.1. Polypol auf Rohstoff- und Reservenmarkt

6.2.2.2. Duopol auf dem Rohstoffmarkt 


\section{Einführung}

\subsection{Einordnung und Problemstellung der Arbeit}

Die Betrachtung der Allokation von Naturressourcen gehörte nicht in dem Ausmaß wie die Betrachtung der Allokation und die Entlohnung der Arbeit von Anbeginn der Volkswirtschaftslehre zu ihrem Forschungsgegenstand. Zwar gab es bei den Klassikern der Nationalökonomie in abgewandelter Form eine "These der Knappheit natürlicher Ressourcen", deren Implikationen der Nationalökonomie der damaligen Zeit den Ruf einer "dismal science" einbrachte1), jedoch befaßten sie sich nicht mit dem Allokationsproblem der optimalen intertemporalen Nutzung der Naturressourcen.

Erst $H$. Hotelling führte für einen gegebenen, nichterneuerbaren Ressourcenbestand eine formale Analyse zur intertemporalen Allokation durch. Mit Hotelling's mittlerweile schon klassisch zu nennendem Aufsatz "The Economics of Exhaustible Resources" kann der Beginn der "neueren" Ressourcentheorie angesetzt werden. Hotelling schreibt:

"Contemplation of the world's disappearing supplies of minerals, forests, and other exhaustible assets has let to demands for regulation of their exploitation. The feeling that these products are now too cheap for the good of future generations, that they are being selfishly exploited at too rapid a rate, and that in consequence of their excessive cheapness they are being produced and consumed wastefully has given rise to the conservation movement." 2)

1) Zu denken ist hier an die Veröffentlichungen von Th. Malthus (1798),

2) Hotelling (1931), S. 137. 
Benutzte L. Gray (1914) vor Hotellıng noch die verbale Analyse und eıne Vielzahl numerischer Beispiele, so konnten sich R. Gordon (1967) und R. G. Cummings (1969) der von Hotelling bereitgestellten Analyseinstrumente bedienen, um dessen Ansatz zu erweitern.

Im Anschluß an die Arbeiten von Gordon und Cummıngs begann dann ab 1970 die Anzahl der Beiträge zur Ressourcenokonomie schnell anzuwachsen. Gegen Ende der siebziger Jahre verstärkte sich das Wachstum der Literaturbeiträge noch einmal.

Gegenstand der okonomischen Theorie der erschöpfbaren Naturressourcen ist es, mittels eines Preissystems die intertemporal optımale Nutzung eines Ressourcenbestandes zu beschreiben. Alle Literaturbeiträge zur Ressourcentheorie nehmen Bezug auf Hotellings Artikel "The Economics of Exhaustible Resources" und gelangen durch Variation und/oder Erweiterung der Modellannahmen zu allgemeineren bzw. neuen Aussagen über die intertemporale Allokation natürlicher Ressourcen.1)

Der Großteil der Literaturbeiträge analysiert Bestimmungsfaktoren der intertemporalen Ressourcenallokation unter Sicherheit. Seltener sind die Arbeiten zu dem Bereich der intertemporalen Ressourcenallokation unter Risiko.

Eine übersichtsartige Darstellung aller bisher in der Literatur diskutierten Bestımmungsfaktoren führt zu der folgenden Systematik ausgewählter Literaturbeıträge:

1) Zu einer detaillıerten Übersicht siehe Peterson/Fisher (1977), Devarajan/Fisher (1981), Siebert (1981a). 
(1) Abbaukosten, Lagergrößen und Diskontraten bei vollkommener Konkurrenz (Schmalensee (1976), Levhari/Liviatan (1977), Hartwick (1978), Kemp/Long (1980b), Stollery (1981a), Siebert (1982a,b));

(2) Wiederverwendungstechnologien (Weinstein/Zeckhauser(1974), Smith, V.L. (1977), Jaeger (1980));

(3) Kuppelproduktionstechnologien (Pindyck (1981b));

(4) technischer Fortschritt (Kamien/Schwartz (1977b, 1978), Davison (1978), Pethig (1980));

(5) Bevölkerungswachstum (Ingham/Simmons (1975), Cigno (1979), Dasgupta /Mitra (1980));

(6) Forschung und Entwicklung (Dasgupta/Gilbert/Stiglitz (1980));

(7) Ressourcensubstitute (Smith, V.L. (1974), Hanson (1977b, 1978), Sauter-Servaes (1980));

(8) Staatliche Eingriffe über Steuern (Lusky (1975), Burness (1976), Anderson (1977), Sinn (1980a)); staatliche Eingriffe über Preiskontrollen (Lee (1979), Tietenberg (1980), Birchenhall/Grout (1980), Stollery (1981b), Kalt (1981), Kalt/Otten (1981));

(9) internationaler Handel (Vousden (1974), Dasgupta/Eastwood/Heal (1978), Kemp/Okoguchi (1979), Suzuki/Ogawa (1979), Kemp/Long (1980a), Siebert (1984));

(10) Marktformen

- vollkommene Konkurrenz (Weinstein/Zeckhauser (1975), Hoel (1978b), Dasgupta/Stiglitz (1980b), Kemp/Long (1982));

- monopolistische Konkurrenz (Sweeney (1977), Dasgupta/Stiglitz (1980a)); 
- Monopol (Stıglitz (1976), Gilbert/Goldman (1978), Dasgupta/Stıglitz(1980b), Dasgupta/Gilbert/Stıglitz (1980));

- Duopol (Hoel (1978b), Dasgupta/Stıglitz (1980b));

- Oligopol (Lewis/Schmalensee (1980a, b), Eswaran/Lewis (1980), Gottwald/Güth (1980), Gottwald (1981));

(11) langfristige Verträge (Roberts (1980));

(12) fehlende bzw. nicht exklusive Eigentumsrechte (Smith, V.L. (1968), Cummıngs (1969), Khalatbarı (1977), Kemp/Long (1980C));

(13) Art der Vergabe von Abbaurechten (Sutinen (1980));

(14) Rısiko über1)

- Eigenschaften des Ressourcenlagers wie Bestand und Qualität (Kemp/Long (1976), Gilbert (1977, 1979a, b), Loury (1978), Heal (1979), Robson (1979), Pindyck (1980), Deshmukh/Pliska (1980));

- Abbaukosten oder die Kosten eines Ressourcensubstitutes (Hanson (1977a), Hoel (1978a, 1980));

- Eigentumsrechte eines Ressourcenlagers (Long (1975));

- Zeitpunkte, zu denen Ressourcensubstitute bzw. backstop-Technologien zur Verfügung stehen (Dasgupta/Heal (1974), Kamien/ Schwartz (1978), Hoel (1980), Dasgupta/Stiglitz (1980b, 1981));

- Nachfragen zukünftiger Perioden (Weınstein/Zeckhauser (1975), Lewis (1977), Dasgupta/Eastwood/Heal (1978), Pindyck (1980, 1981a, b), Hillman/Long (1982)).

1) Zu einer Übersicht über die Betrachtung des Risikos in der Ressourcentheorie siehe Long (1983). 
Die vorliegende Arbeit beschrankt sich auf die mikrookonomischen Aspekte der Ressourcentheorie. Ausgehend vom optımalen Angebotsverhalten einer Abbauunternehmung wird in partiellen Gleichgewichtsmodellen die intertemporale Allokation be verschiedenen institutionellen Gegebenheiten untersucht. Die Problemkreise der erschopfbaren Ressourcen in makroökonomıschen Modellen sind nıcht Gegenstand dieser Arbeit.1)

In der bisherigen Diskussion zur Ressourcentheorie stand die Analyse von Marktallokationen unter vollkommener Information im Vordergrund. Ein vollkommenes und vollständiges System von Märkten generiert das erforderliche Preıssystem. D.h., für das betrachtete Gut Rohstoff existieren neben Gegenwarts- und Zukunftsmärkten auch Risikomärkte.2) Gibt man die Annahme eines vollständigen Marktsystems auf, Zukunfts- und Risikomärkte seien nicht vorhanden, dann fallen Allokationsmechanismen aus und rufen bei den Wirtschaftssubjekten bestimmte Reaktionen hervor. Kaum diskutiert wurden in der Ressourcentheorie bisher die Allokationswirkungen von Institutionen, die sich als Substitute fehlender Zukunftsmärkte (Marktsubstitute) herausgebildet haben. Insbesondere sind hier die Vertragsvereinbarungen in den verschiedenen Ausprägungen zunennen.

Die Nutzung der erschöpfbaren Naturressourcen läßt sich mit folgendem Prozeß grob beschreiben: 3 )

1) Zu einem Überblick dieser Fragestellungen siehe Stiglitz (1979). Dasgupta/Heal (1974), Mitra (1978), Heal (1980), Siebert (1983) seien hier beispielhaft für viele Beiträge genannt.

2) Der Terminus "vollkommen" bezieht sich dabei auf die effiziente Funktionsweise eines Marktes und der Terminus "vollständig" meint die Existenz von Märkten für jeden Zeitpunkt ( Gegenwarts-und Zukunftsmärkte ) bzw. jeden Zustand der Welt (Risikomärkte). In diesem Sinne werden die Begriffe vollkommen und vollständig bspw. auch von Leland (1978), S.414: "...perfect and complete contingency claim markets..." und Long (1983), S.71 : "...complete and perfect set of futures markets..." benutzt.

3) Vgl. hierzu Brown/Field (1979), S.224. 
Schaubild 1.1: Die Nutzung erschopfbarer Ressourcen

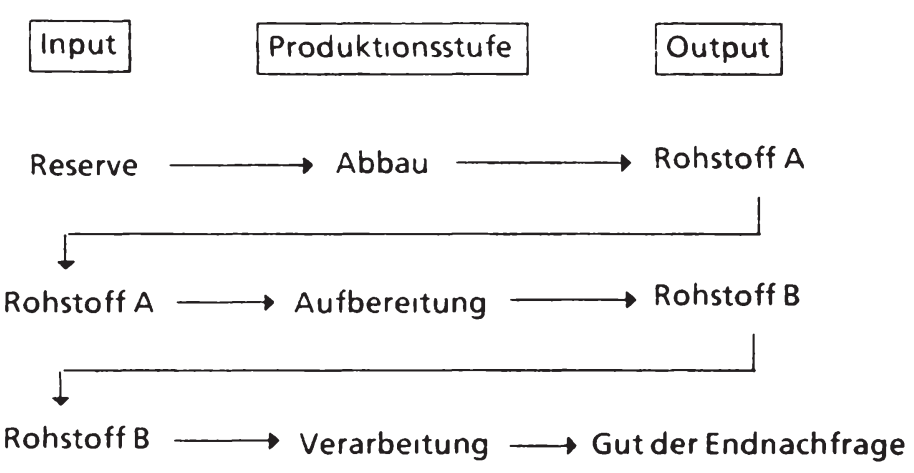

Zwischen den einzelnen Produktionsstufen Abbau, Aufbereitung und Verarbeitung findet im allgemeınen der Übergang von einem zu einem anderen Wirtschaftssubjekt statt. Faßt man die Produktionsstufen der Aufbereitung und Verarbeitung zusammen, so kann man für den Ressourcenhandel dreı Märkte unterscheiden:

Den Reservenmarkt, den Rohstoffmarkt und den Rohstoffproduktmarkt (Schaubild 1.2). 
Schaubild 1.2: Ressourcenmärkte

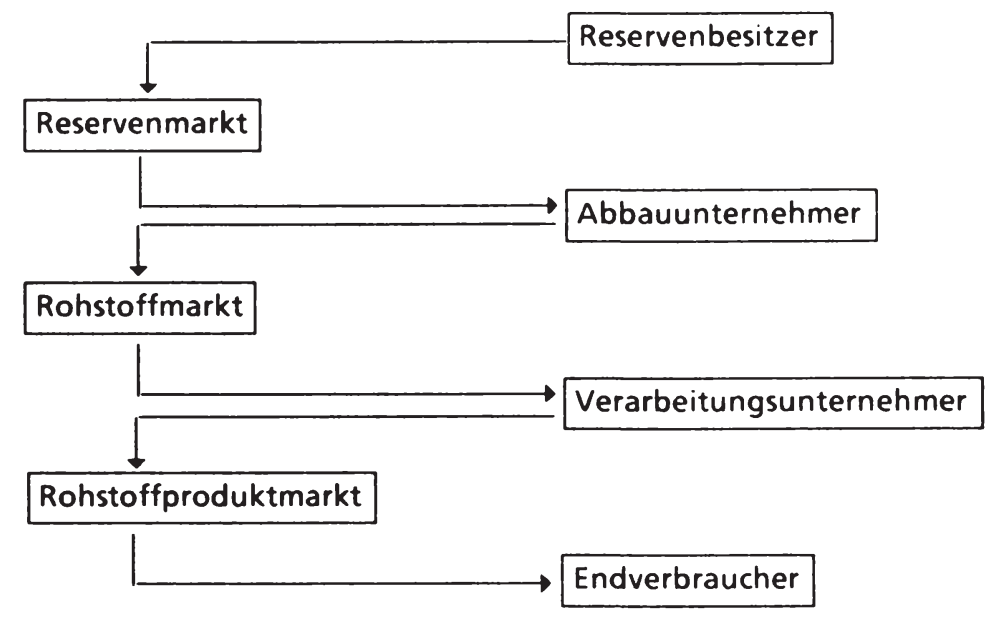

Auf dem Reservenmarkt bieten die Eigentümer der Reservenläger ihre Bestände zur Nutzung an und die Abbauunternehmen fragen diese Bestände zur Förderung nach. Der Rohstoffmarkt dient zum Handel der noch nicht aufbereiteten und verarbeiteten Rohstoffe zwischen dem Abbau- und dem Verarbeitungsunternehmen. Schließlich geht die Ressource als Produkt an den Endverbraucher über.

Üblicherweise wird in der Ressourcentheorie unterstellt, daß das Abbauunternehmen im Besitz des Reservenlagers ist. Ein dem Rohstoffmarkt vorgelagerter Markt ist unter diesen Voraussetzungen nicht zu betrachten. Für die Analyse von Vertragsvereinbarungen zwischen den Abbauunternehmen und den Reservenbesitzern ist es jedoch erforderlich, einen solchen Markt zu unterstellen. Die Betrachtung der Allokationswirkungen von 
langfristıgen Lieferverträgen zwischen Abbau- und Verarbeitungsunternehmen kann wieder vom Reservenmarkt abstrahieren. In dieser Arbeit wird sowohl der Reservenmarkt als auch der Rohstoffmarkt in die Betrachtung mit einbezogen, da Antworten auf die folgenden Fragen gegeben werden sollen:

(1) Wie andert sich das Abbauverhalten in der Zeit, wenn Risiko über die Nachfrage zukünftiger Perioden berücksichtıgt wird?

(2) Hat Risiko unter verschiedenen Marktformen unterschiedliche Allokationseffekte?

(3) Welche Effekte bewirken langfristige Lieferverträge in Ressourcenmodellen?

(4) Welche Auswirkungen haben die unterschiedlichen Ausgestaltungen von lang fristigen Lieferverträgen?

(5) Welche Effekte haben Terminkontrakte und Optionskontrakte in Ressourcenmodellen?

(6) Haben unterschiedliche Vertragsvereinbarungen zwischen Ressourcenbesitzern und Abbauunternehmern auch unterschiedliche Allokationseffekte (Konzession, Abbaumengenteilung, Dienstleistungsvertrag)?

Anhand dieser Fragen ist zu erkennen, daß es das Ziel dieser Arbeit ist, einen Beitrag zur Analyse der Funktionsweise von Märkten und Marktsubstituten bei der intertemporalen Allokation von nichterneuerbaren Naturressourcen zu liefern. Dazu wird in systematischer Weise wie folgt vorgegangen werden:

Nach einer Klassifikation des Ressourcen begriffes anhand unterschiedlicher in der Literatur vorzufindender Definitionen (Abschnitt 1.2.) gibt Kapitel 2 eınen Uberblick über dıe intertemporale Allokation erschöpfbarer Ressourcen im vollständigen Marktsystem (d.h. unter Sicherheit) und erweitert die 
Diskussion um alternative Zielformulierungen (Umsatzmaxımıerung, Nutzenmaximierung, Pressdifferenzierung).

Laßt man die Abwesenheit von Zukunftsmärkten zu (Risıko uber zukunftıge Nachfragen ), so hat dies je nach Risikoverhalten Auswirkungen auf die intertemporale Ressourcenallokation. Kapitel 3 führt diese Betrachtungsweise durch. Diskutiert werden die Allokationswirkungen unterschiedlichen Rısikoverhaltens bei verschiedenen Marktformen (Polypol, Monopol, Oligopol).

Auf fehlende Zukunftsmärkte werden die Wirtschaftssubjekte mit dem Ziel reagieren, die für sie dadurch entstehenden Informationsmängel und Risiken zu reduzieren oder gar zu beseitigen. In Kapitel 4 wird aufgezeigt, mittels welcher Institutionen die Wirtschaftssubjekte auf fehlende Zukunftsmärkte reagieren.

Die ressourcentheoretische Analyse der in Kapitel 4 diskutierten Institutionen nimmt Kapitel 5 in Zweiperıodenmodellen vor. Es werden die Allokationswirkungen von "langfristigen" Lieferverträgen, von Terminkontrakten und von Optionskontrakten ermittelt.

Kapitel 6 spezialisiert sich dann auf die Analyse der Allokationswirkungen von Vertragsvereinbarungen in Mehrperiodenmodellen. Zwischen Kapitel 5 und 6 besteht der Unterschied nicht nur in der Anzahl der Perioden bzw. in der Unterscheidung zwischen diskreter und stetiger Analyse, sondern es besteht auch ein qualitativer Unterschied hinsichtlich der Betrachtung des Risikos. Im Zweiperiodenmodell existiert für den Abbauunternehmer ein Risiko nur über die Nachfrage der zweiten Periode. Dagegen ist im Mehrperiodenmodell auch die Nachfrage in der ersten Periode unsicher. Kapitel 5 zeigt die Allokationswirkungen auf, wie man sie für eine closed-loop Lösung in Mehrperiodenmodellen erhalten würde, ohne auf die stochastische dynamische Optimierung zurückzugreifen. Kapitel 6 dagegen gıbt die Allokationswirkungen als open-loop Lösung an.

Eine zusammenfassende Darstellung der Ergebnısse liefert Kapıtel 7. 


\subsection{Definition und Klassifikation des Allokationsobjektes Naturressource}

Ressourcen können im weitesten Sinne definiert als Guter definiert werden.1) Spezieller definiert Lecomber2) : "..., resources being a general term used to cover all inputs to production, not only land, minerals and fuels but also labour and capital and even foreign exchange."

Innerhalb dieser Definitionen sind dann Naturresourcen zu verstehen als direkt aus der Umwelt ( Natur) gewinnbare, menschliche Bedürfnisse direkt oder indirekt befriedigende Mittel.

Lecomber meint dazu3): "The definitive feature of natural resources is, that they are provided by nature, not by man."

Ebenso definieren Smith und Krutilla4) naturliche Ressourcen als "... all the original elements that comprise the earth's natural endowements."

Nicht so breit definiert Howe5): "Natural resources could refer to all the living and nonliving endowment of the earth, but traditional usage confines the term to naturally ocurring resources and systems that are useful to humans or could be under plausible technological, economic, and social circumstances."

Den Naturressourcen stehen begrifflich gegenüber die nicht von der Natur bereitgestellten, nicht aus der Natur entnommenen Ressourcen ( NN -Ressourcen ). Hierunter einzuordnen wären die von Lecomber genannten Ressourcen Arbeit und Kapital.

1) Vgl. Yuchtman/Seashore (1967), S.900.

2) Lecomber (1979), S.1.

3) a.a.O.

4) Smith/Krutilla (1979), S.5.

5) Howe (1979), S.1. 
Rohstoffe, definiert als Mittel mit stofflichen Eigenschaften (fest, flüssig, gasförmig), bılden zusammen mit Boden, Wasser 1) und Energie die Menge der Naturressourcen. Energie ist zu verstehen als nichtstoffliche Naturressource, die fähig ist, Arbert zu leısten.

Energiearten in diesem Sinne existieren in der Natur als solare und geothermische Energie. Wasserkraft, Gezeiten-oder Windenergie sınd eınzuordnen als indirekte solare Energien2).

Fallen unter den Begriff der Energien nur nichtstoffliche erneuerbare $\mathrm{Na}$ turressourcen, so hat man bei den Rohstoffen zu unterscheiden zwischen erneuerbaren und nichterneuerbaren Rohstoffen.

Ein Rohstoff heißt erneuerbar (nicht erneuerbar), wenn für ihn eine (keine) Rohstoffeinheit mit positiver Regenerationsrate existiert.3) Die Regenerationsrate des Gesamtbestandes kann positiv, null oder negativ sein. Positive Regenerationsraten von nahezu null (wie bspw. bel den fossilen Rohstoffen über lange Zeiträume) sind für die Zeiträume des Menschen bedeutungslos; die zugehörıgen Rohstoffe zählen als nichterneuerbar.

Mit diesen Begriffen sind all jene Eigenschaften einer Ressource angesprochen, die sie selbst hervorbringt (natürlich, stofflich, erneuerbar). Nicht eindeutig den inhärenten Eigenschaften einer Ressource zuzuordnen ist die Wiederverwertbarkeit. Denn die Wiederverwertbarkeit ist nicht nur abhängig von der Art der Ressource, sondern auch von der Art ihrer Verwendung und dem Stand der Technologien zur Wiederverwertung.

Schaubild 1.3 zeigt eine Gegenúberstellung der vorherigen Erörterungen.

1) Boden und Wasser hier nicht verstanden als Rohstoff, sondern als Lieferant von Ressourcendiensten.

2) Hierzu bspw. Grathwohl (1978), S.51.

3) Bspw. existiert für den Rohstoff Holz als regenerative Einheit der Baum. 
Schaubild 1.3: Klassıfikation des Ressourcenbegriffes

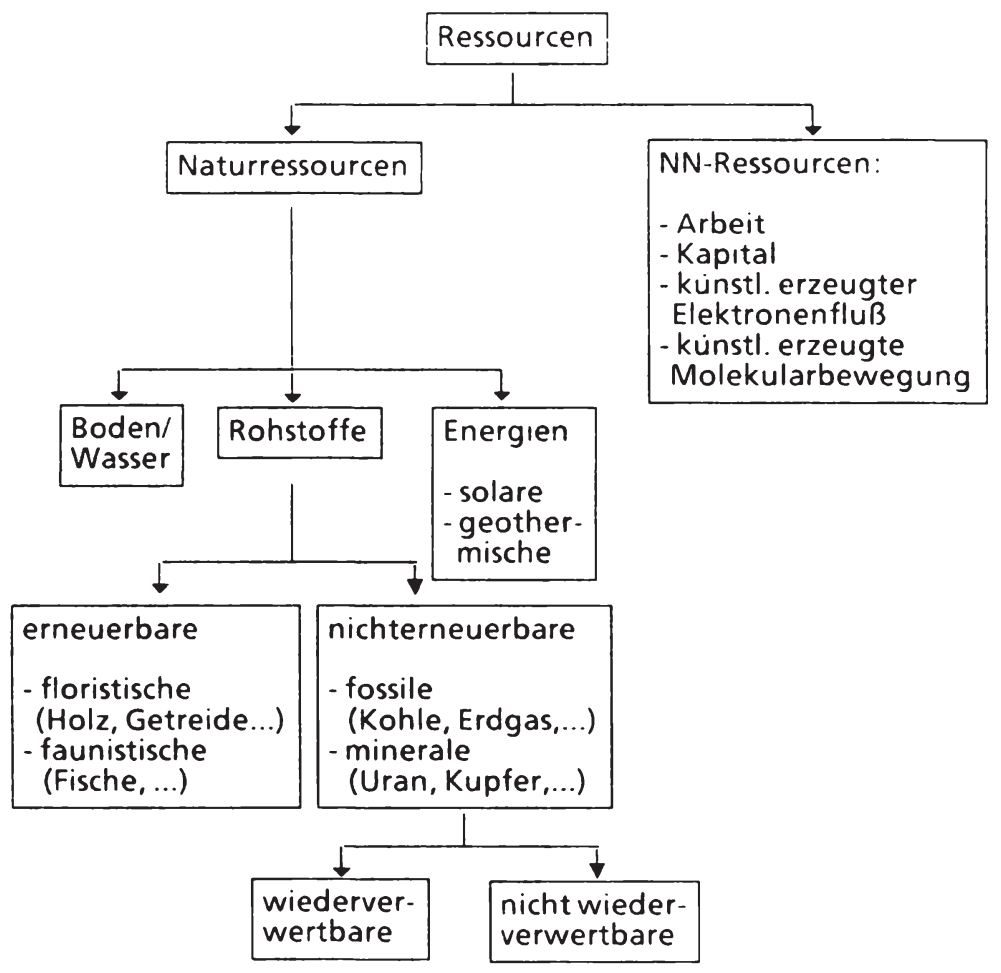


Bei Howe i) findet man eıne Aufzahlung von "Hauptklassen" der naturlichen Ressourcen, mit der er seine Definıtion für den heutigen Sprachgebrauch auf umweltliche und okologische Systeme erweitert :

Ackerland; Wälder mit ihren Produkten und Diensten; Land fur àsthetische, regenerative und wissenschaftliche Zwecke; Mineralien (fossile und nichtfossile); erneuerbare nichtmineralische Energiequellen, Wasser und Assımilationskapazitäten.

Howe unterscheidet bei seıner Aufzählung nicht zwischen der Ressource und den von ihr durch Nutzung erhaltbaren Diensten. Eine solche Unterscheidung sollte aber gemacht werden, da sich dann eine Klassifikation des Ressourcenbegriffes nicht mit der Aufzählung einer Vielzahl von Nutzungsmöglichkeiten zu befassen braucht.

Bei Common und Pearce findet sich eine Klassifikation zum Begriff der Ressource.2) Sie klassifizieren die Ressourcen unter dem Aspekt der Konsequenzen einer heutigen Ressourcennutzung für zukünftige Generationen und kommen zu den Unterscheidungsmerkmalen Energien und Materialien einerseits und Bestände und Ströme andererseits:

(1) Energieressourcenbestände (SER)

haben einen festen Bestand über ihren gesamten Abbauzeitraum. Bei positiver Abbaurate sind ihre Läger innerhalb eines bestimmten Zeit raumes erschöpft. Ein Recycling ist hier nicht möglich. Über die Abbaurate wird die intertemporale Allokation bestımmt.

1) Howe (1979), S.1f.

2) Common/Pearce (1973), S.296. 
Schaubild 1.4: Ressourcenklassıfikation nach Common/Pearce

\begin{tabular}{|c|c|c|}
\hline & Energien & Materıalien \\
\hline Bestande & $\begin{array}{l}\text { (1) } S_{\text {tock }} E_{\text {nergy }} \text { Resources } \\
\text { (Kohle, Erdgas, Erdöl,...) }\end{array}$ & $\begin{array}{l}\text { (2) Stock Material Resources } \\
\text { (Blei, Kupfer,...) } \\
\text { (4) Stock Renewable Resources } \\
\text { (Meeresressourcen, } \\
\text { Arbeit,...) }\end{array}$ \\
\hline Ströme & $\begin{array}{l}\text { (3) Flow Energy Resources } \\
\text { (Sonnenenergie, } \\
\text { Wasserkraft,...) }\end{array}$ & \\
\hline
\end{tabular}

(2) Materialressourcenbestände (SMR)

besitzen ebenfalls einen festen Gesamtbestand, unterscheiden sich jedoch von den SER aufgrund ihrer anderen Entstehungsart und Nutzung. Ein Recycling ist bei diesen Ressourcen möglich. Neben der Abbaurate hat hier die Wiederverwendungstechnologie Einfluß auf die intertemporale Allokation.

(3) Energieressourcenströme (FER)

sind gekennzeichnet durch einen festen Zustrom pro Periode. Da sie in einem nennenswerten Ausmaß nicht lagerungsfähig sind bzw. Lagerungsmöglichkeiten nıcht existieren, sind die FER für eine intertemporale Allokation nicht relevant. 
(4) Erneuerbare Ressourcenbestände (SRR)

haben pro Zeitpunkt eınen festen Bestand, der mit eıner variablen Rate erneuerungsfähig ist. Die natürliche ( bei Meeresressourcen ) bzw. gelenkte (bei Fischfarmen) Regenerationsrate bestımmt zusammen mit der Nutzungsrate die Verfügbarkeit der SRR in der Zeit.

Die Common/Pearceschen Ressourcenklassen sind in das Schaubild 1.3 wie folgt einzuordnen:

SER $\rightarrow$ nichterneuerbare fossile Rohstoffe

SMR $\rightarrow$ nichterneuerbare minerale Rohstoffe

FER $\rightarrow$ Energien 1)

SRR $\rightarrow$ erneuerbare Rohstoffe.

Von den der Ressource innewohnenden Eigenschaften zu unterscheiden sind die der Ressource zugewiesenen Eigenschaften. Zugewiesen werden der Ressource diese Eigenschaften von dem die Ressource nutzenden ökonomischen System. Es sind die Eigenschaften,

- privatisierbar oder öffentlich

- bei der Produktion notwendig oder nicht notwendig

- erschöpfbar oder unerschöpfbar

zu sein.

Nichtausschließbarkeit von der Nutzung und Nichtrivalität bei der Nutzung einer Ressource begründen ihre Eigenschaft, eine öffentliche Ressource zu sein.

Geht eine Ressource in einen Produktionsprozeß ein und wäre die Ausbringung dieses Prozesses null, so wird die Ressource als notwendig für diesen Prozeß bezeichnet.

1) Verstanden als nichtstoffliche Naturressource. SER und FER werden in der energietheoretischen Literatur auch bezeichnet als nichterneuerbare bzw. erneuerbare Primärenergieträger. 
Cummings definiert "an exhaustible resource as a natural resource the supply of which is not naturally replenished." ") Er benutzt als Merkmal der Erschopfbarkeit eine Regenerationsrate von null. Eine Regenerationsrate $\left(r_{r}=0\right)$ von null ist weder hınreıchend noch notwendig für den vollstandigen Abbau eınes Rohstofflagers. Erst eine positive Abbaurate $\left.\left(r_{a}>0\right) 2\right)$ ergibt zusammen mit $r_{r}=0$ eine notwendige Bedingung für die Erschöpfung des Lagers eıner nichterneuerbaren Ressource.3) Denn mit der Änderung von Präferenz- und/oder technologischen Strukturen andert sich auch die Struktur der genutzten Rohstoffe, so daß bisher abgebaute Rohstoffarten nicht mehr weiter genutzt werden und mit dem verbleibenden Lagerbestand weiter verfügbar sind.

Leiter scheint den Begriff der Erschöpfbarkeit auf d eselbe Art zu interpretieren, wenn er schreibt: "As human wants and social objectives change, the meaning of 'resources' is modified. "4) Rohstoffe, die trotz positiven Lagerbestands nicht mehr genutzt werden, fallen aus seiner Ressourcendefinition5) heraus und sind damit nicht mehr Gegenstand der Diskussion um Erschöpfbarkeiten.

Für die Analyse dieser Arbeit wird stets von einer privatisierbaren, produktionsnotwendigen, erschöpfbaren Naturressource ausgegangen.

1) Cummings (1969), S.201.

2) Für erneuerbare Ressourcen ist zu fordern $r_{r}<r_{a}$.

3) Dies ist auch hinreichend. Sie wäre nicht hinreichend, falls $r_{a}=0$ bevor das Lager abgebaut ist.

4) Leiter (1976), S.9f.

5) "Resources consists of those elements in the environment which make it possible to satisfy individual and group objectives." Leiter (1976), S.9. 


\section{Die intertemporale Allokation bei vollständigen Zukunfts- märkten}

Dieser Abschnitt stellt die Allokationsergebnisse der verschiedenen Marktformen unter vollkommener Information der handelnden Wirtschaftssubjekte gegenuber. Ausgegangen wird von der Marktform des Polypols, dem die des Angebotsmonopols und die einer oligopolistischen Angebotsstruktur bei polypolistischer Nachfrageseite folgen. Unter der Marktform des Polypols werden neben der Gewinnmaximierung alternative Zielsetzungen der Abbauunternehmungen in ihren Allokationswirkungen diskutiert. Die Zielsetzung der Nutzenmaximierung einer Unternehmensführung, die nicht Eigentümer der Firma ist, wird in Abschnitt 6.2.3. bei der Modellierung einer Vertragsvereinbarung wieder aufgegriffen.

Für das Angebotsmonopol wird der Fall der Preisdifferenzierung diskutiert. Zwischen dem Allokationsmuster der Preisdifferenzierung bei vollständigen Zukunftsmärkten und dem Allokationsmuster der Preisdifferenzierung bei fehlenden Zukunftsmärkten ist das Allokationsmuster eines Angebotsmonopols bei fehlenden Zukunftsmärkten mit langfristigen Lieferverträgen einzuordnen (Abschnitt 6.1.3.4.).

Das optimale langfristıge Verhalten der Abbauunternehmer wird im konventionellen Sinn als einstufiger Entscheidungsprozeß betrachtet. D.h. sämtliche Marktteilnehmer stellen zu Beginn des Planungs- (Abbau-) zeitraumes einen verbindlichen Entscheidungsplan auf. In Termini der dynamischen Optimierung gesprochen, werden open-loop Lösungen betrachtet. Gegenüber den Marktformen des Polypols und des Monopols, sind für Oligopole Lösungskonzepte der dynamischen Spieltheorie anzuwenden. Gewählt wird das des open-loop-Nash-Gleichgewichtes in einem nichtkooperativen Nichtnullsummen-Differentialspiel.1)

1) Gottwald (1981) bzw. Guth/Gottwald (1980) betrachteten bisher als einzige closed-loop Lösungen für Oligopolsituationen in der Ressourcentheorie. 
Abschließend wird ein zweistufiges Marktkonzept vorgestellt, wie es in einem spateren Abschnitt (6.2.) be fehlenden Zukunftsmarkten wieder zugrundegelegt wird.

\subsection{Das Polypol}

Am weitaus haufigsten diskutiert wird in der ressourcentheoretischen Literatur die Marktform der vollstandıgen Konkurrenz. Bereits Hotelling (1931) machte Aussagen über die zeitliche Entwicklung der gleıchgewichtigen Mengen und Preise. 1) Das Angebotsverhalten resultiert aus der Zielsetzung der Gewinnmaximierung. Weinstein/Zeckhauser (1975) zeigen, daß die vollständige Konkurrenzlösung auch die sozial optimale Losung ist. Cropper/ Weinstein/Zeckhauser (1978) machen dazu eine differenzierte Aussage: Die vollstandige Konkurrenzlösung ist optimal, wenn die Abbautechnologien für alle Firmen identisch sind, bzw. wenn bei unterschiedlichen Abbautechnologien die Abbaurechte oder die Technologien gehandelt werden können.

Den weiteren Betrachtungen dieses Abschnittes wird die Marktform der vollständigen Konkurrenz mit einer gewınnmaximierenden Angebotsseite als Referenzmodell vorangestellt.

Für die Analyse der intertemporalen Allokation unter vollständıger Konkurrenz wird im allgemeınen von den folgenden Annahmen ausgegangen:2)

Es gibt eine Vielzahl Ressourcenläger, von denen jedes im Besitz eines Abbauunternehmens ist.

1) Bei Gordon (1967), S.277; Nordhaus (1973), S.531; Weinsteın/Zeckhauser (1975), S.373 findet man diese Aussagen bestätigt.

2) Von Autor zu Autor ändern sich diese Annahmen bzgl. der Kostenfunktion, der Nachfragefunktion, der Existenz eines Ressourcensubstitutes, etc. 
Alle Unternehmen bauen die Ressource mit identischen Kosten $C(q(t)) a b$. Für die Kostenfunktion gilt: $d C / d q=C^{\prime}(q(t))>0$ und $d^{2} C / d q^{2}=C^{\prime \prime}(q(t))>0$.

Die Marktpreise der Rohstoffe $p(t)$ sind für die Abbauunternehmer vorgegebene Daten ohne Risiko.

Auf einem perfekten Kapitalmarkt können die Abbauunternehmer ihre Erträge zu eınem Zinssatz $\delta$ anlegen. Dieser Zinssatz ist im Zeitablauf konstant und wird von den Unternehmern als Diskontrate benutzt.

Jedes Unternehmen wählt den Abbaupfad $\{q(t)\}$, der die Zielfunktion maxımiert unter der Nebenbedingung des gegebenen, bekannten Ressourcenbestandes.

Hinsichtlich der Zielfunktion 1) soll unterschieden werden, ob der Eigentümer selbst die Abbauentscheidung trifft (gewinnorientierter Ansatz), oder ob der Eigentümer Manager beschäftigt, die für ihn die Abbauentscheidungen treffen (umsatz- und nutzenorientierter Ansatz).

Ein Preıspfad für die Entwicklung der Gleichgewichtspreıse ist zu definieren als jener Preisvektor, bei dem zu jedem Zeitpunkt im Abbauzeitraum die Nachfrage nach Rohstoffen gleich dem Angebot an Rohstoffen ist.

Für die Nachfrage nach Rohstoffen soll gelten $p(t)=N(q(t))$ mit $d N / d q=N^{\prime}(q(t))<0$.

Um in den verschiedenen Modellvarianten endliche Abbauzeiträume zu erhalten, werden für den Vergleich der Allokationsergebnisse durchgàngig lineare Nachfragefunktionen unterstellt, also $d^{2} \mathrm{~N} / \mathrm{dq}^{2}=\mathrm{N}^{\prime \prime}(q(t))=0$.

1) Vgl. hierzu Blattner (1976), S.242. Dort werden die verschiedenen Firmentypen anhand ihrer Zielfunktionen gekennzeichnet als eigentums-, umsatz-oder nutzenorientierte Firma. 


\subsubsection{Der gewinnorientierte Ansatz: Gewinnmaximierung der} Eigentümer

Unter den gemachten Annahmen lautet für den gewinnmaximierenden Abbauunternehmer das Optimierungsproblem:

(2.1)

$$
\begin{aligned}
& \max _{(q(t), T)}{ }_{0} S^{\top}[p(t) q(t)-C(q(t))] e^{-\delta t d t} \\
& \text { u.d.B. } \quad R_{0} \geq{ }_{0} S^{\top} q(t) d t, R_{0} \text { gegeben, } q(t) \geq 0 .
\end{aligned}
$$

Dies führt über dıe notwendigen Bedingungen

(2.2)(i) $\quad\left[p(t)-C^{\prime}(q(t))\right] e^{-\delta t}-\lambda$

(2.2)(ii) $\quad R_{0}-{ }_{0} \int^{\top} q(t) d t$

$$
\begin{aligned}
& \begin{cases}=0 & q(t)>0 \\
& \text { für } \\
\leq 0 & q(t)=0\end{cases} \\
& \left\{\begin{array}{lll}
=0 & \lambda>0 \\
& \text { fur } & \\
\geq 0 & \lambda=0
\end{array}\right.
\end{aligned}
$$

(2.2)(iii) $\quad[p(T) q(T)-C(q(T))] e^{-} \delta T-\lambda q(T)=0$

zu den Eigenschaften der Angebotsseite des Rohstoffmarktes. 1)

1) Es wird hier nicht explızit unterschieden zwischen den bei dieser Marktform infinitesimal kleınen Großen für den einzelnen Anbieter und den aggregierten Großen. 
Plant der Unternehmer die Rohstoffmenge $\mathrm{q}(\mathrm{t})$ abzubauen und anzubieten, dann wird der Marktpreis die dabeı anfallenden "Grenzkosten des Abbaus" decken. Ist diese Gleichheit nicht gegeben, dann ist es optımal, nichts abzubauen, $q(t)=0$. Die "Grenzkosten des Abbaus" beschrànken sich be der langfristigen Gewinnmaximierung jedoch nicht auf die marginalen $A b$ baukosten $\mathrm{dC} / \mathrm{dq}$, sondern berücksichtigen marginale Kosten für die durch den Abbau entgangenen alternativen intertemporalen Verwendungsmöglichkeiten.

$\lambda$ gibt den Gegenwartswert der Opportunitätskosten der Nutzung der Ressource an. Dieser ist konstant und im Optımum gleich dem Gegenwartswert des Grenzgewinnes aus der Abbautätigkeit:

$$
p(t)-C^{\prime}(q(t))=\lambda e^{\delta t}
$$

Das Abbauprogramm ist optimal, wenn der Ressourcenbestand mit einer Rate abgebaut wird, bei der die daraus erzielbaren Grenzgewinne im gleichen Ausmaß wachsen wie Ertráge am Kapitalmarkt ( Hotelling-Regel):

$$
\left[d p / d t-C^{\prime \prime}(q(t))(d q / d t)\right]\left[p(t)-C^{\prime}(q(t))\right]-1=\delta
$$

Der Abbauunternehmer ist dann indifferent zwischen dem Abbau und der Konservierung der Ressource.

Zur Ermittlung der Preis- und Mengenpfade ist es erforderlich, die Nachfrage explizit einzubeziehen:

$$
p(t)=N(q(t)) \text { mit } N^{\prime}(q(t))<0 \text { und } N^{\prime \prime}(q(t))=0 .
$$

Für die Marktgleichgewichte im Abbauzeitraum $[0, T]$ ergeben sich die Zeitpfade

$$
\begin{aligned}
& d q / d t=\delta\left[p(t)-C^{\prime}(q(t))\right]\left[N^{\prime}\left(q(t)-C^{\prime \prime}(q(t))\right]-1<0\right. \text { und } \\
& d p / d t=N^{\prime}(q(t)(d q / d t)>0 .
\end{aligned}
$$


Die Fordermengen nehmen dem Betrage nach mit zunehmenden Raten ab. Der Marktpreıs der Ressource wächst mit einer Rate kleiner als $\delta .{ }^{1)}$

Beı dem unterstellten Nachfrageverlauf gibt es eınen "choke-off" -Preis2) $p(T)$, fur den gilt $p(T)=(0)$.

Da auch in T Marktgleichgewicht herrscht, muß beim Preis $p(T)$ das Angebot ebenfalls null seın. Der Abbau wird eingestellt. Aus 2.2(ii) folgt für $\lambda>0$ die Erschopfung des Ressourcenbestandes im Zeitraum [0,T].

Im optimalen Erschöpfungszeitpunkt T muß auch die Transversalitätsbedingung 2.2 (iii) gelten : $p(T) q(T)-C(q(T))=\lambda e^{\delta T} q(T)$.

Hieraus folgt $q(T)=0$ oder $q(T)>0$. Falls $q(T)>0$ ist, folgt weiter mit 2.2 (i) die Gleichheit von Durchschnitts- und Grenzkosten des Abbaus im Endzeitpunkt der Abbautätigkeit. Fúr $q(T)=0$ liefert diese Transversalitätsbedingung keine zusätzliche Information.

Eine Darstellung der Mengen- und Preispfade erfolgt im Vergleich mit den Ergebnissen erlösmaximierender Abbauunternehmen am Ende des nächsten Abschnittes (Schaubild 2.1).

1) Dies folgt aus 2.6 wegen $C^{\prime}(q(t)) / p(t)>0>C^{\prime \prime}(q(t)) / N^{\prime}(q(t))$, denn $(\mathrm{dp} / \mathrm{dt}) / \mathrm{p}=\delta\left[1-\mathrm{C}^{\prime}(\mathrm{q}(\mathrm{t})) / \mathrm{p}(\mathrm{t})\right]\left[1-\mathrm{C}^{\prime \prime}(\mathrm{q}(\mathrm{t})) / \mathrm{N}^{\prime}(\mathrm{q}(\mathrm{t}))\right]$. Vgl. zu diesen $\mathrm{Er}$ gebnissen bspw. Hotelling (1931), S.141, Gordon (1967), S.277, Weinstein/Zeckhauser (1975), S.379, Siebert (1983), S.78f.

2) In einigen Modellen wird die Annahme eines "choke"-Preises explizit gemacht. Diesen Preis kann man sich als bestimmt denken durch die Kosten eines Ressourcensubstitutes oder einer backstop-Technologie. Vgl. bspw. Lewis/Schmalensee (1980), S.477, die für die Existenz von Gleıchgewichten in Oligopolsituationen diese Annahme treffen. 


\subsubsection{Der umsatzorientierte Ansatz: Umsatzmaximierung durch} Manager

Von Baumol'1) wurde das Konzept der umsatzorientierten Firma in die wettbewerbstheoretische Diskussion eingebracht, um der Tatsache Rechnung zu tragen, daß größere Unternehmen unter der Leitung eines Managements Umsatzmaximierung betreiben. Dies wird unter der Nebenbedingung eines befriedigenden Gewinnes getan. Von einem direkten Einfluß auf die Unternehmensentscheidungen sind die Eigentümer ausgeschlossen. Die Eigentümer lassen das Management solange gewähren, wie die Renditeerwartungen durch eine entsprechende Gewinnerzielung befriedigt werden

Der Umsatz eines Abbauunternehmens im Abbauzeitraum $[0, T]$ ermittelt sich zu

$$
W={ }_{0} \int^{\top} p(t) q(t) e^{-\delta t} d t .
$$

Diesen gilt es zu maximieren unter den Nebenbedingungen des gegebenen Ressourcenbestandes $R_{0}$ und des von den Eigentümern geforderten Mindestgewinnes $G_{0}$, der zur Ausschüttung kommt.

$$
\begin{aligned}
& \max _{(q(t), T)} W \\
& \text { u.d.B. } \quad R_{0} \geq{ }_{0} \int^{\top} q(t) d t, R_{0} \text { gegeben, } q(t) \geq 0 \\
& \quad G_{0} \leq{ }_{0} \int^{\top}[p(t) q(t)-C(q(t))] e-\delta t d t, G_{0} \text { gegeben }
\end{aligned}
$$

Aus dem Optimierungsansatz folgen uber die Lagrangefunktion

1) Baumol (1959), S.45-53. 


$$
L=W+\lambda\left[R_{0}-{ }_{0} S^{\top} q(t) d t\right]+\mu\left\{{ }_{0} S^{\top}[p(t) q(t)-C(q(t))] e^{-\delta t d t}-G_{0}\right\}
$$

die notwendigen Bedingungen

(2.9)(i) $p(t) e^{-\delta t-\lambda+\left[p(t)-C^{\prime}(q(t))\right] \mu e^{-} \delta t} \begin{cases}=0 & q(t)>0 \\ & \text { für } \\ \leq 0 & q(t)=0\end{cases}$

(2.9)(ii) $\quad R_{0}{ }_{-0} S^{\top} q(t) d t$

$$
\left\{\begin{array}{lll}
=0 & & \lambda>0 \\
& \text { für } & \\
\geq 0 & \lambda=0
\end{array}\right.
$$

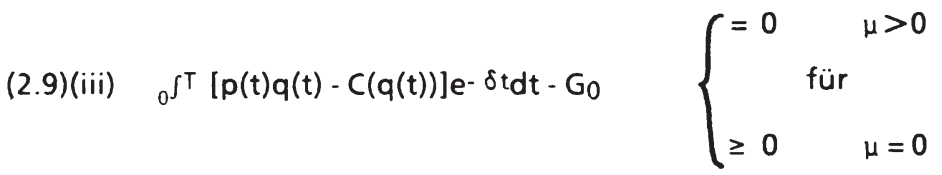
(2.9)(iv) $p(T) q(T) e^{-\delta T}-\lambda q(T)+\mu[p(T) q(T)-C(q(T))] e^{-} \delta T=0$.

Würden die Eigentümer keinen Mindestgewinn fordern bzw. diesen so klein wählen, daß die Nebenbedingung nicht bindet $(\mu=0)$, dann hätte man ein Abbauverhalten wie in einem Modell ohne Abbaukosten.

Die Manager setzen dann den Gegenwartswert des Marktpreises gleich dem Schattenpreis der Ressource:

(2.10) $\quad p(t) e^{-\delta t}=\lambda$.

Der Marktpreis wächst mit der Diskontrate. In den Marktgleichgewichten des Abbauzeitraumes gilt mit der Nachfragefunktion 2.4

(2.11) $d p / d t=\delta p(t)>0$ und

(2.12) $\quad \mathrm{dq} / \mathrm{dt}=\delta p(\mathrm{t}) \mathrm{N}^{\prime}(\mathrm{q}(\mathrm{t}))^{-1}<0$. 
Anders dagegen wählen die Manager das Abbauprogramm, wenn die Gewinnrestriktion bindet. Für $\mu>0$ folgt

$$
p(t)=(1+\mu)^{-1} \lambda e^{\delta t}+\mu(1+\mu)^{-1} C^{\prime}(q(t)) .
$$

Der Marktpreis der Ressource bildet sich aus Opportunitätskosten der Ressourcennutzung und Grenzkosten des Abbaus, jedoch nun korrigiert mit $\mu$, den Opportunitätskosten der Gewinnausschüttung. Eine marginale Erhohung der Gewinnausschüttung mindert den Barwert des maxımalen Umsatzes um $\mu$.

Die Marktgleichgewichte liefern für die Entwicklung der Rohstoffpreise und Abbaumengen

$$
\begin{aligned}
d q / d t= & \delta\left[p(t)-\mu(1+\mu)^{-1} C^{\prime}(q(t))\right] . \\
& {\left[N^{\prime}(q(t))-\mu(1+\mu)^{-1} C^{\prime \prime}(q(t))\right]^{-1}<0 . }
\end{aligned}
$$

$$
d p / d t=N^{\prime}(q(t))(d q / d t)>0 .
$$

Ein Vergleich der Wachstumsraten der gleichgewichtigen Marktpreise aus 2.6 und 2.15 zeigt, daß die Marktpreise unter der Annahme gewinnmaximierender Abbauunternehmen mit einer geringeren Rate wachsen als die Marktpreise unter der Annahme umsatzmaximierender Manager, die einer Gewinnrestriktion unterliegen.1) Entfällt die Gewinnrestriktion, dann wächst der Marktpreis mit der größeren Rate des Zinssatzes $\delta$.

Für die Abbauprofile bedeutet dies, daß eine gewinnmaximierende Angebotsseite einen gegebenen Ressourcenbestand langsamer erschöpft als ei-

1) Dies folgt aus $0<\mu(1+\mu)^{-1}<1$ und

$$
\begin{aligned}
& {\left[1-\mu(1+\mu)^{-1} C^{\prime}(q(t)) p(t)-1\right]\left[1-\mu(1+\mu)^{-1} C^{\prime \prime}(q(t)) N^{\prime}(q(t))^{-1}\right]^{-1}>} \\
& \left.\left[1-C^{\prime}(q(t)) p(t)\right)^{-1}\right]\left[1-C^{\prime \prime}(q(t)) N^{\prime}(q(t))^{-1}\right]^{-1} .
\end{aligned}
$$


ne umsatzmaximierende Angebotsseite, die Gewinnrestriktionen zu beachten hat und diese wiederum langsamer abbaut als ohne Gewinnrestriktıonen.

Worin liegt die Ursache für dieses unterschiedliche Abbauverhalten? Warum baut ein umsatzmaximierendes Unternehmen einen gegebenen Ressourcenbestand schneller ab als ein gewinnmaximierendes Unternehmen?

Ein gewinnorientiertes Abbauunternehmen steuert seine Abbaumengen so, daß bei gegebener Nachfrage sein Grenzgewinn in Gegenwartswerten zu jeder Perıode gleich ist. Dies erreicht es bei steigenden Grenzkosten mit sinkenden Abbaumengen.

Das ohne Gewinnrestriktion umsatzmaximierende Abbauunternehmen hingegen bezieht die Abbaukosten nicht in die Zielfunktion mit ein und realisiert dadurch eine größere Steigerung der Marktpreise als das gewinnmaximierende Unternehmen. Die konservierende Funktion von Abbaukosten tritt hier nicht ein. Die stärker steigenden Marktpreise führen zu einer früheren Erschöpfung der Ressource. Unterliegt die Unternehmensführung einer Gewinnrestriktion, dann erfüllen die Opportunitätskosten der Gewinnausschüttung (teilweise) die Funktion der in der Zielfunktion nicht berücksichtigten Abbaukosten; die Gewinnrestriktion wirkt konservierend auf das Abbauprogramm. Je höher der geforderte Mindestgewinn, desto größer ist der Schattenpreis der Gewinnausschüttung, und umso mehr nähert sich das Abbauprogramm dem eines gewinnorientierten Unternehmens.

Die Interpretation der Transversalitätsbedingung 2.6 (iv) ist gleich der von 2.2 (iii).

In Schaubild 2.1 kennzeichnen die Indices G, WG bzw. W die verschiedenen Pfade aufgrund der Annahmen Gewinnmaximierung, Umsatzmaximierung mit bzw. ohne Gewinnrestriktion. 
Schaubild 2.1: Die intertemporale Ressourcenallokation bei verschiedenen Zielsetzungen der Abbauunternehmen
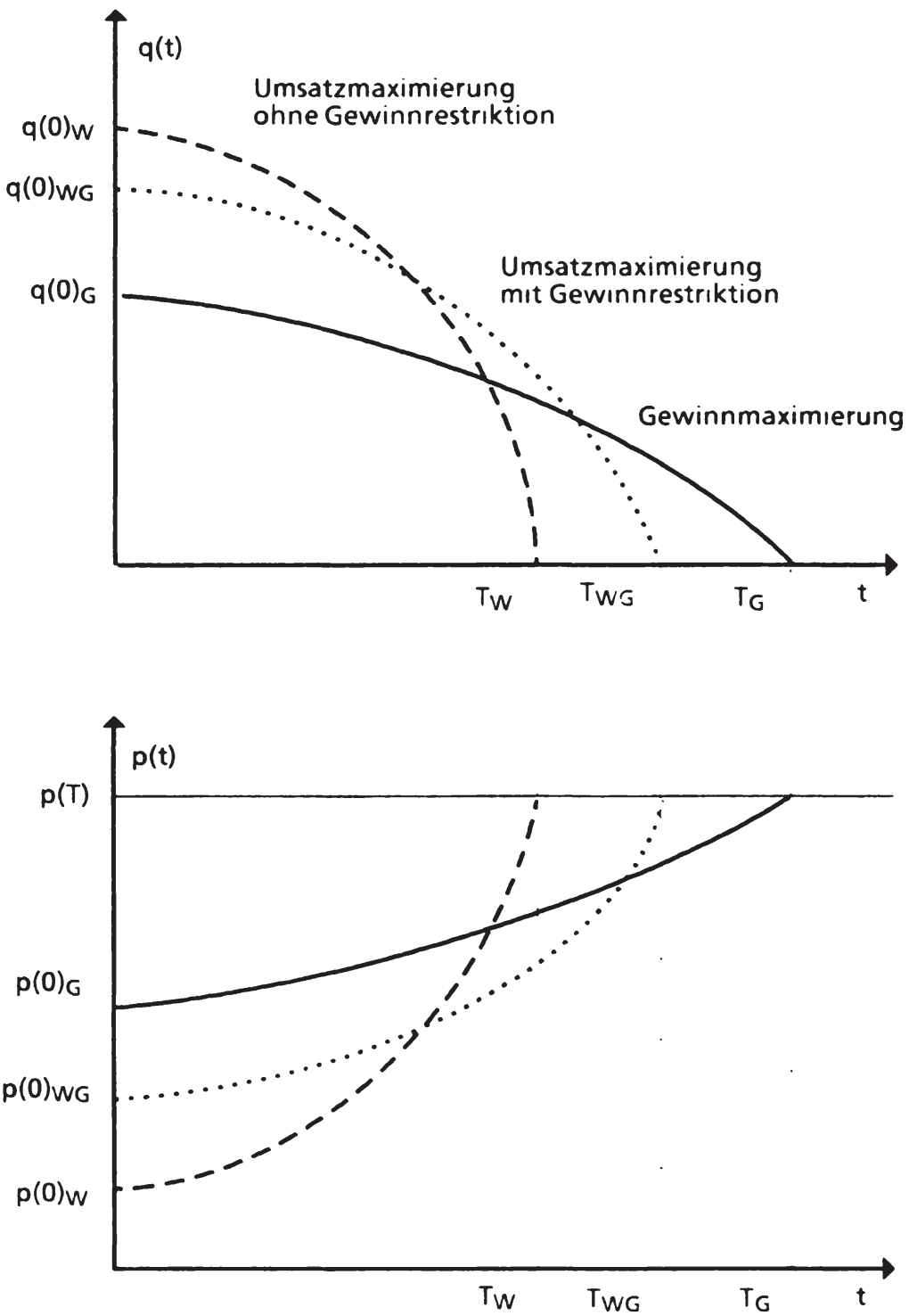


\subsubsection{Der nutzenorientierte Ansatz: Nutzenmaximierung der} Manager

Den nutzenorientierten Ansatz versteht Williamson 1) als eine Verallgemeinerung des Baumolschen Ansatzes der Umsatzmaximierung. Anstelle des Eıgentumers der Unternehmung handelt auch hier ein angestelltes Management. Als Zielfunktion maximiert die Unternehmensfuhrung (der Manager) jedoch nicht den Gewinn oder den Umsatz, sondern den Nutzen V aus der erhaltenen Entlohnung $A(t)$ für die Dienstleistung der Tätigkeit Unternehmensführung:

$$
V=V[A(t)] \text {, mit dV/dA }=V_{A^{\prime}}>0 \text { und } d^{2} V / d A^{2}=V_{A}{ }^{\prime \prime}<0 .
$$

Eine Erhöhung der Entlohnung steigert den Nutzen des Managers mit abnehmendem Grenznutzen.

Der oder die Eigentümer des Abbauunternehmens fordern von dem Manager einen Mindestgewinn $G_{0}$ über den Zeitraum der Abbautätigkeit. In jeder Periode resultiert aus der Abbautätigkeit ein Gewinn von

$$
G(t)=p(t) q(t)-C(q(t))-A(t)
$$

der an die Eigentümer ausgeschüttet wird.

Die Entlohnungen $A(t)$ des Managers sind in dieser Gewinndefinition für die Eıgentumer als periodenfixe, von der Ausbringungsmenge unabhängige Kosten aufzufassen.

Mit $\mathrm{G}_{0}$ soll die Forderung der Eigentümer nach eınem Mindestgewinn geschrieben werden als

1) Williamson (1971), S. 379f. Zum statıschen Ansatz vgl. Williamson (1972), wo ein ahnlicher Ansatz als "staff model" bezeichnet wird (S. 69). 


$$
G_{0} \leq{ }_{0} \int^{T} G(t) e^{-i t} t d t .
$$

Unter der Nebenbedingung der Bestandsrestriktıon für die abzubauende Ressource und der Gewinnrestriktion maxımiert der Manager nun in Gegenwartswerten den gesamten Nutzen aus der Entlohnung für die Unternehmensführung wàhrend der Abbautätigkeit.

$$
\begin{aligned}
& \max _{(q(t), G(t), J)}{ }_{0} \int^{\top} V[A(t)] e^{-}-v t d t . \\
& \text { u.d.B. } \quad R_{0} \geq{ }_{0}^{T} q(t) d t, R_{0} \text { gegeben, } q(t) \geq 0, G(t) \geq 0 \\
& \qquad G_{0} \leq{ }_{0} \int^{\top} G(t) e-s t d t, G_{0} \text { gegeben. }
\end{aligned}
$$

Über die Lagrangefunktion

$$
\left.L={ }_{0} S^{\top} V[A(t)] e^{-\cdots t d t}+\lambda\left[R_{0}-{ }_{0} \int^{\top} q(t) d t\right]+\mu\left[{ }_{0}{ }^{\top} G(t) e^{-s t d t}-G_{0}\right] 1\right)
$$

folgt

$$
V_{A^{\prime}}\left[p(t)-C^{\prime}(q(t))\right] e^{-s t}-\lambda \quad \begin{cases}=0 & q(t)>0 \\ & \text { für } \\ \leq 0 & q(t)=0\end{cases}
$$

(2.19)(ii) $\quad-V_{A^{\prime}} e^{-i t}+\mu$

(2.19)(iii) $R_{0}-{ }_{0} \int^{\top} q(t) d t$

$$
\begin{cases}=0 & G(t)>0 \\ \text { für } & \\ \leq 0 & G(t)=0\end{cases}
$$$$
\begin{cases}=0 & \lambda>0 \\ \text { für } & \\ \geq 0 & \lambda=0\end{cases}
$$

1) Die Ermittlung der notwendigen Bedingungen erfolgt unter Beachtung von $A(t)=p(t) q(t)-C(q(t))-G(t)$. 
(2.19)(iv) ${ }_{0} \int^{\top} \mathrm{G}(\mathrm{t}) \mathrm{e}^{-1, t} \mathrm{dt}-\mathrm{G}_{0}$

$$
\begin{cases}=0 & \mu>0 \\ \text { für } & \\ \geq 0 & \mu=0\end{cases}
$$

$(2.19)(v) \quad V[A(T)] e^{-o ̈ T}-\lambda q(T)+\mu G(T) e-\delta T=0$

$\mu$ ist wieder zu interpretieren als Schattenpreis einer ausgeschütteten Gewinneınheit. Dieser Schattenpreis gıbt die Minderung des maximalen Gegenwartswertes der gesamten Periodennutzen der Unternehmensführung an, wenn die Eigentümer eine Mehrausschüttung der Gewinne um eine marginale Einheit fordern. $\mu$ ist im Optimum gleich dem Grenznutzen des Managers aus seiner Entlohnung und in Gegenwartswerten konstant.

(2.20) $\quad V_{A^{\prime}}=\mu$

Aus 2.19 (i) wird für den in Gegenwartswerten ebenfalls konstanten Schattenpreis der Ressourcennutzung erhalten

$$
V_{A}^{\prime}\left[p(t)-C^{\prime}(q(t))\right] e^{-\delta t}=\lambda
$$

Der Schattenpreis für die Ressourcennutzung ist gleich dem Gegenwartswert des in Nutzeneinheiten bewerteten Grenzgewinnes einer Ressourceneinheit.

Zur Ermittlung des markträumenden Presspfades für die Rohstoffe und des zugehörigen Abbauprofiles wird wieder die Nachfragefunktion 2.4 unterstellt.

Die Differentiation von 2.20 und 2.21 nach der Zeit liefert zwei Bestimmungsgleichungen für dq/dt und dG/dt: 1)

1) Aus 2.20 folgt $V_{A}$ " $(d A / d t)=0$, und weiter $d A / d t=0$ wegen $V_{A}{ }^{\prime \prime}<0$; es ist $d A / d t=\left[N^{\prime}(q(t)) q(t)+p(t)-C^{\prime}(q(t))\right](d q / d t)-d G / d t$, so daß gilt $d G / d t=\left[N^{\prime}(q(t)) q(t)+p(t)-C^{\prime}(q(t))\right](d q / d t)$. 


$$
\begin{aligned}
& d G / d t=d q / d t\left[N^{\prime}(q(t)) q(t)+p(t)-C^{\prime}(q(t))\right] \\
& d q / d t=\delta\left[p(t)-C^{\prime}(q(t))\right]\left[N^{\prime}(q(t))-C^{\prime \prime}(q(t))\right]-1<0 .
\end{aligned}
$$

Ferner gilt

$$
d p / d t=N^{\prime}(q(t))(d q / d t)>0 .
$$

Ein Vergleich von 2.23 mit 2.5 führt zu der Aussage, daß unter Sicherheit ein dienstleistendes Management den Ressourcenbestand mit demselben Abbauprofil erschöpft wie ein gewinnorientierter Abbauunternehmer dies in eigener Regie tun würde.

Aus 2.22 ist die zeitliche Entwicklung der Gewinnausschüttung abzuleiten.

$$
d G / d t><0 \Leftrightarrow-n(t)><p(t)\left[p(t)-C^{\prime}(q(t))\right]^{-1}, d a d q / d t<0 .
$$

Tendenziell ist die Aussage möglich, daß das Management die Gewinnausschüttungen steigen läßt, solange die Rohstoffe im unelastischen Bereich der Nachfrage abgesetzt werden. Die Gewinnausschüttungen sinken, wenn die Rohstoffe im elastischen Bereich der Nachfrage vermarktet werden.

Beginnt die Abbautätigkeit und damit die Vermarktung im unelastischen Nachfragebereich, so werden die Gewinnausschüttungen zuerst zunehmen, um dann gegen Ende der Abbautätigkeit abzunehmen.

Zusätzliche Informationen über die Eigenschaften des optimalen Zeitpunktes zur Einstellung der Abbautätigkeit erhält man aus 2.19 (v) für $q(T)>0$. Es folgt mit 2.19(i), (ii) für $G(T), \lambda>0$ die Gleichheit

$$
\begin{aligned}
& V[A(T)]+V_{A^{\prime}} G(T)=V_{A^{\prime}}\left[p(T)-C^{\prime}(q(T))\right] q(T) \Leftrightarrow \\
& V[A(T)] q(T)^{-1}+V_{A^{\prime}} G(T) q(T)^{-1}=V_{A^{\prime}}\left[p(T)-C^{\prime}(q(T))\right] .
\end{aligned}
$$

Der Durchschnittsnutzen des Managements plus dem in Nutzeneinheiten bewerteten, ausgeschütteten Durchschnittsgewinn , ist gleich dem in Nutzeneinheiten bewerteten Grenzgewinn der zuletzt abgebauten Ressourceneinheit. 


\subsection{Das Monopol}

Nach dem Polypol ist das Monopol die Marktform, welche ebenfalls häufig in der ressourcentheoretischen Literatur diskutiert wird. Auch hier ist Hotelling (1931) als erster zu nennen, der Preis- und Mengenpfade des Abbaus beschrieben hat.

Neben den weiteren theoretischen Beiträgen von bspw. Stiglitz (1976), Kamien/ Schwartz (1977) und Sweeney (1977) existieren auch eine Vielzahl empirischer Arbeiten zu dieser Marktform. Denn im Vergleich zu der Marktform des Polypols findet man die des Angebotsmonopols schon eher in der Wirklichkeit. Bobrov/ Kudrle (1976) analysieren Ressourcenkartelle der OPEC. Modelle des Weltölmarktes betrachten bspw. CremerMeitzman (1976), Hnyilicza/Pindyck (1976), Gately/Kyle/Fischer (1977) und Pindyck (1978).

Eine Darstellung und Würdigung der empirischen Arbeiten soll hier nicht erfolgen. Die Betrachtung bleibt auf die theoretische Diskussion beschränkt.

\subsubsection{Ressourcenabbau bei antizipiertem Nachfrageverhalten}

Der Monopolist baue mit derselben Kostenfunktion wie die Polypolisten einen Ressourcenbestand ab, der dasselbe Ausmaß wie die Summe der Ressourcenbestände der polypolistischen Abbauunternehmer haben soll. Weiter soll für Vergleichszwecke unterstellt werden, daß der Monopolist mit derselben Diskontrate wie die Polypolisten kalkuliert.1)

1) Hier könnte auch die Annahme gemacht werden, daß der Monopolist mit einer geringeren Diskontrate kalkuliert, da er einen besseren $\mathrm{Zu}$ gang zu den Kapitalmärkten hat als der Polypolist; vgl. Stiglitz (1976), S.659. 
Der vom Monopolısten in seınem Kalkül berucksichtigte Nachfrageverlauf seı reprasentıert durch die Nachfragefunktion 2.4 .

Das Optımıerungsproblem hat damıt das folgende Aussehen

(2.27) $\quad \max { }_{0}^{\int}[N(q(t)) q(t)-C(q(t))] e^{-\delta} t d t$ $(q(t), T)$

u.d.B. $R_{0} \geq{ }_{0} \int^{\top} q(t) d t, R_{0}$ gegeben, $q(t) \geq 0$.

An notwendigen Bedingungen werden aus der Lagrangefunktion

$L={ }_{0} S^{\top}[N(q(t)) q(t)-C(q(t))] e^{-\delta t d t}+\lambda\left[R_{0}-{ }_{0} S^{\top} q(t) d t\right]$

erhalten:

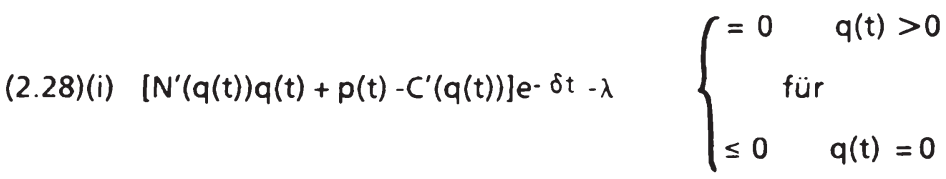

(2.28)(ii) $R_{0}-{ }_{0} \int^{\top} q(t) d t$

$$
\left\{\begin{array}{lll}
=0 & \lambda>0 \\
\text { für } & \\
\geq 0 & \lambda=0
\end{array}\right.
$$

(2.28)(iii) $[p(T) q(T)-C(q(T))] e^{-} \delta T-\lambda q(T)=0$

Für den Monopolisten ist jenes Abbauprofil optimal, bei dem der Gegenwartswert seiner Grenzgewinne konstant ist:

(2.29) $\quad p(t)+N^{\prime}(q(t)) q(t)-C^{\prime}(q(t))=\lambda e^{\delta t}$. 
Da der Grenzerlös des Monopolisten von dem des Polypolisten verschieden ist (Marktpreıs), wird der Monopolıst ım allgemeinen ein anderes Abbauprofil realisieren.

Mit der Preiselastizitat der Nachfrage $n(t)=[N(q(t)) / q(t)] / N^{\prime}(q(t))$ folgt aus 2.29

$$
p(t)[1+n(t)-1]-C^{\prime}(q(t))=\lambda e^{i t}
$$

Verglichen mit der vollständigen Konkurrenz ist der Marktpreis und dessen zeitliche Veränderung nun auch von der Elastızität der Nachfrage bzw. der Form der Nachfragefunktıon abhängig. 1)

Aus 2.30 folgt $p(t)[1+\eta(t)-1]>0$, d.h. $n(t)<-1$ für $p(t)>0$. Solange der Monopolist am Rohstoffmarkt anbietet, sinkt die Preiselastizitat der Nachfrage nicht auf den Betrag eins. Der Monopolist bietet nur im elastischen Bereich der Nachfrage an.

Die Differentiation von 2.29 nach der Zeit führt zu

$$
\begin{aligned}
d q / d t= & \delta\left\{p(t)\left[1+n(t)^{-1}\right]-C^{\prime}(q(t))\right\} \\
& \left.\left\{N^{\prime}(q(t))[1+n(t))^{-1}-(d n / d t)(q(t) / n(t))-C^{\prime \prime}(q(t)) / N^{\prime}(q(t))\right]\right\}-1 \\
d q / d t< & <0
\end{aligned}
$$

und zu

$$
\begin{aligned}
d p / d t= & \delta\left\{p(t)\left[1+\eta(t)^{-1}\right]-C^{\prime}(q(t))\right\} \\
& {\left[1+\eta(t)-1-(d \eta / d t)(q(t) / n(t))-C^{\prime \prime}(q(t)) / N^{\prime}(q(t))\right]-1 }
\end{aligned}
$$

1) Vgl. hierzu bspw. Weinstein/Zeckhauser (1975), Stiglitz (1976), Sweeney (1977), Gottwald (1981), S.101 oder Siebert (1983), S.245f; aber auch schon Hotelling (1931), S.147. 
War in vollständiger Konkurrenz die Wachstumsrate des. Preises kleiner als die Diskontrate, so kann der Marktpreis des Rohstoffes nun auch mit einer großeren Rate wachsen:

$$
\begin{aligned}
&(d p / d t) p(t)^{-1}= \delta\left[1+n(t)^{-1}-C^{\prime}(q(t)) p(t)-1\right] \\
& {\left.\left[1+n(t)^{-1}-(d n / d t)(q(t) / n(t))-C^{\prime \prime}(q(t)) N^{\prime}(q(t))\right)^{-1}\right]^{-1} } \\
&(d p / d t) p(t)-1>\left.\left.<\delta \Leftrightarrow 0><C^{\prime}(q(t)) p(t)\right)^{-1}-C^{\prime \prime}(q(t)) N^{\prime}(q(t))\right)^{-1}- \\
&(d n / d t)(q(t) / n(t))
\end{aligned}
$$

Im Falle konkaver Nach fragefunktionen $\left(N^{\prime}(q(t))<0\right.$ und $\left.N^{\prime \prime}(q(t)) \leq 0\right)$ ist $\left.d \eta / d q=-N^{\prime \prime}(q(t)) N(q(t))\left[N^{\prime}(q(t))\right)^{2} q(t)\right]^{-1}+\left[N^{\prime}(q(t))-N(q(t))\right]\left[N^{\prime}(q(t)) q(t) 2\right]^{-1}>0$.

Für isoelastische Nachfragefunktionen ist $d \eta / d q=0.1$ ) In beiden Fällen werden Steigerungsraten der Marktpreise realisiert, die geringer sind als die Diskontrate.

Wann wird der Monopolist eine Preıssteigerungsrate von mehr als $\delta$ erzielen? Für zeitlich invariante Nachfrageverläufe2) folgt aus der Bedingung

$$
\begin{aligned}
(d p / d t) p(t)^{-1}>\delta \Leftrightarrow(d \eta / d t)(q(t) / n(t)) & >C^{\prime}(q(t)) p(t)^{-1}- \\
C^{\prime \prime}(q(t)) N^{\prime}(q(t))^{-1} & >0
\end{aligned}
$$

Schließt man Abbaukosten aus der Betrachtung aus, dann ist

$\left(2.33^{\prime \prime}\right) \quad(d p / d t) p(t)-1>\delta \Leftrightarrow d \eta / d t<0$

und weiter $d \eta / d t<03) \Leftrightarrow-N^{\prime \prime}(q(t)) q(t) N^{\prime}(q(t))^{-1}>1-n(t)^{-1}>0$.

1) Kamien/Schwartz (1977) leiten eine solche Nachfrage aus einer linear homogenen Cobb-Douglas Produktionsfunktion ab.

2) Um ein $\mathrm{dp} / \mathrm{dt}>\delta$ zu erhalten, diskutiert Gottwald (1981), S.103 den Fall einer zeitlich varianten linearen Nachfragefunktion $p(t)=a(t)$ $b q(t)$ mit $b>0$ und da/dt $>\delta a(t)$.

3) $d \eta / d q<0$ aber $n(t)<-1$ im Abbauzeitraum. 
Die strenge Konvexıtat der Nachfragefunktion $\left(N^{\prime \prime}(q(t))>0\right)$ ist nicht hinreichend für eıne Preıssteıgerung $d p / d t>\delta$. Zusätzlıch muß die Elastizität der ersten Ableitung der Nachfrage $\left[-d N^{\prime} / d q\right]\left[q(t) / N^{\prime}(q(t))\right]$ den um eins vermehrten Wert des Kehrwertes der Preıselastızität der Nachfrage übersteigen.1)

Für die weitere Betrachtung wird wieder ein linearer Nachfrageverlauf zugrundegelegt. Die Linearität der Nachfragefunktion ist eine hinreichende Bedingung für $(d p / d t) / p(t)<\delta$.

Es stellt sich die Frage, ob der Monopolist nun schneller oder langsamer abbaut als der Polypolist.

Abweichend von der in der Literatur üblichen Darstellungsweise erhält man aus 2.28 (i) ohne die Benutzung der Preiselastizität der Nachfrage :

$$
d q / d t=\delta\left[N^{\prime}(q(t)) q(t)+p(t)-C^{\prime}(q(t))\right]\left[2 N^{\prime}(q(t))-C^{\prime \prime}(q(t))\right]^{-1}<0
$$

bzw.

$\left(2.32^{\prime}\right) \quad \mathrm{dp} / \mathrm{dt}=\mathrm{N}^{\prime}(\mathrm{q}(\mathrm{t}))(\mathrm{dq} / \mathrm{dt})>0$.

Der Vergleich mit 2.5 und 2.6 zeigt, daß unter der Marktform des Polypols ein gegebener Ressourcenbestand schneller erschöpft wird als im Monopol.

Einen weiteren Unterschied zum Polypol kann man bei der Betrachtung der Transversalitätsbedingung feststellen, die für den Fall $q(T)>0$ nun ebenfalls von der Nachfrageelastizität beeinflußt wird.

Gleichung 2.28 (iii) ist erfüllt für $q(T)=0$ oder $q(T)>0$.

1) Gottwald bezeichnet den beschleunigten Abbau der Ressource durch den Monopolisten aufgrund $\mathrm{dp} / \mathrm{dt}>\delta$ als einen "pathologischen Fall"'. Vgl. Stiglitz (1976), S.659 oder Sweeney (1977), S.134f, wo hinreichende Bedingungen für konservierendes Abbauverhalten angegeben werden. 
Der Polypolist hat den Abbau eingestellt, wenn sein Bestand erschöpft war oder wenn die Gleichheit zwischen Durchschnitts- und Grenzkosten erreicht war.

Der Monopolist dagegen stellt den Abbau des Ressourcenbestandes nicht bei der Gleichheit von Durchschnitts- und Grenzkosten ein, sondern er baut weiter ab, bis die Grenzkosten die Durchschnittskosten um einen bestimmten Wert unterschreiten. 1) Aus 2.28 (iii) folgt für $q(T)>0$ mit 2.30

$$
-p(T) / n(t)=C(q(t)) / q(T)-C^{\prime}(q(T)) .
$$

\subsubsection{Ressourcenabbau und Preisdifferenzierung bei räumlich getrennten Märkten}

Der Monopolist des vorangegangenen Abschnittes sei nun in der Lage, den Markt für den Absatz seiner Rohstoffe in zwei Teilmärkte zu spalten. Auf den beiden Teilmärkten sollen sich für den Monopolisten die konjekturalen Nachfragen in der folgenden Form ergeben

(2.35)(i) $p_{1}(t)=N_{1}\left(q_{1}(t)\right)$ mit $N_{1}{ }^{\prime}\left(q_{1}(t)\right)<0, N_{1}{ }^{\prime \prime}\left(q_{1}(t)\right)=0$,

(2.35)(ii) $\mathrm{p}_{2}(\mathrm{t})=\mathrm{N}_{2}\left(\mathrm{q}_{2}(\mathrm{t})\right)$ mit $\mathrm{N}_{2}{ }^{\prime}\left(\mathrm{q}_{2}(\mathrm{t})\right)<0, \mathrm{~N}_{2}{ }^{\prime \prime}\left(\mathrm{q}_{2}(\mathrm{t})\right)=0$,

wobei gelten soll $N_{1}{ }^{\prime}\left(q_{1}(t)\right)<N_{2}{ }^{\prime}\left(q_{2}(t)\right)$ und $N_{1}(0)>N_{2}(0)$.

Wie zuvor maximiert der Monopolist den gesamten Gewinn in Gegenwartswerten aus dem Abbau und dem Absatz der Ressource $\left.q(t)=q_{1}(t)+q_{2}(t)\right)$.

1) Auch hier gilt die Gleichheit von Durchschnitts- und Grenzgewinn. 
(2.36)

$$
\begin{aligned}
& \max \\
& \left.\left(q_{1}(t), q_{2}(t), T\right)\right) \\
& \text { u.d.B. } \quad R_{0} \int^{\top}\left[p_{1}(t) q_{1}(t)+p_{0}(t) q_{2}(t)-C(q(t))\right] e^{-} \delta t d t \\
&
\end{aligned}
$$

Über die notwendigen Bedıngungen, $j=1,2$

$(2.37)(i) \quad\left\{p_{j}(t)\left[1+n_{j}(t)^{-1}\right]-C^{\prime}(q(t))\right\} e^{-\delta t-\lambda}\left\{\begin{array}{cc}=0 & q_{j}(t)>0 \\ \text { furr } & \\ \leq 0 & q_{j}(t)=0\end{array}\right.$

(2.37)(ii) $\quad R_{0}-{ }_{0} \int^{\top} q(t) d t$

$$
\left\{\begin{array}{lll}
=0 & \lambda>0 \\
& \text { für } & \\
\geq 0 & \lambda=0
\end{array}\right.
$$

(2.37)(iii) $\left[p_{1}(T) q_{1}(T)+p_{2}(T) q_{2}(T)-C(q(T))\right] e^{-\delta T}-\lambda q(T)=0$

ermıttelt man für die gleichgewichtigen Marktpreise

$$
p_{j}(t)\left[1+n_{j}(t)^{-1}\right]-C^{\prime}(q(t))=\lambda e^{\delta t}, \text { für } j=1,2
$$

Solange auf beıden Märkten gleichzeitig angeboten wird, wächst der Grenzgewinn mit der Diskontrate bzw. sind die laufenden Werte der Grenzerlöse gleich. Für unterschiedliche Preiselastizitäten der Nachfragen auf den Märkten werden jedoch unterschiedliche Preise und unterschiedliche Wachstumsraten realisiert. Aus

$$
p_{1}(t)\left[1+n_{1}(t)^{-1}\right]=p_{2}(t)\left[1+n_{2}(t)^{-1}\right]
$$

folgt $p_{1}(t)><p_{2}(t) \Leftrightarrow n_{1}(t)>_{<} n_{2}(t)$, für $n_{1}(t), n_{2}(t)<-1$. 
Der Monopolist wird zu einem Zeitpunkt, in dem Teilmarkt 1 eine geringere Preiselastizität der Nachfrage aufweist, einen hoheren Rohstoffpreis fordern.

Für die weitere Betrachtung dieses Abschnittes wird auf die Benutzung der Preiselastizität der Nachfrage verzichtet, da sich dadurch die Darstellung vereinfacht.

Zunächst wird die Entwicklung der Preise und Mengen auf beiden Teilmärkten abgeleitet. Anschließend wird der Vergleich zu dem Fall ohne Preisdifferenzierung gezogen.

Die notwendigen Bedingungen 2.37 (i) für $j=1,2$ machen eine Aussage über das Angebotsverhalten des Monopolisten auf den Teilmärkten.

Differenziert man nach der Zeit, so folgt

$(2.40)(i) \quad\left[2 N_{1}{ }^{\prime}\left(q_{1}(t)\right)-C^{\prime \prime}(q(t))\right]\left(d q_{1} / d t\right)-C^{\prime \prime}(q(t))\left(d q_{2} / d t\right)=\delta \lambda e^{\delta t}$

(2.40)(ii) $\left[2 \mathrm{~N}_{2}{ }^{\prime}\left(\mathrm{q}_{2}(\mathrm{t})\right)-\mathrm{C}^{\prime \prime}(\mathrm{q}(\mathrm{t}))\right]\left(d q_{2} / d t\right)-C^{\prime \prime}(\mathrm{q}(\mathrm{t}))\left(d q_{1} / d t\right)=\delta \lambda \mathrm{e}^{\delta \mathrm{t}}$.

Nach 2.39 muß auch gelten 1$)$

$$
N_{1}{ }^{\prime}\left(q_{1}(t)\right)\left(d q_{1} / d t\right)=N_{2}{ }^{\prime}\left(q_{2}(t)\right)\left(d q_{2} / d t\right),
$$

d.h. die Preispfade für die gleichgewichtigen Marktpreise verlaufen mit gleicher Steigung.

2.41 mit 2.40 (i) bzw. (ii) liefert für die Handelsmengen der beiden Märkte:

(2.42)(i) $\quad \mathrm{dq}_{1} / \mathrm{dt}=\delta \lambda \mathrm{e}^{\delta \mathrm{t}} \mathrm{N}_{2}{ }^{\prime}\left(\mathrm{q}_{2}(\mathrm{t})\right) \Omega^{-1}(\mathrm{t})<0$

(2.42)(ii) $\quad \mathrm{dq}_{2} / \mathrm{dt}=\delta \lambda \mathrm{e}^{\delta \mathrm{t}} \mathrm{N}_{1}{ }^{\prime}\left(\mathrm{q}_{1}(\mathrm{t})\right) \Omega^{-1}(\mathrm{t})<0$,

1) 2.39 ist äquivalent der Schreibweise:

$p_{1}(t)+q_{1}(t) N_{1}^{\prime}\left(q_{1}(t)\right)=p_{2}(t)+q_{2}(t) N_{2}{ }^{\prime}\left(q_{2}(t)\right)$; unter Beachtung der Annahme $N_{1}^{\prime \prime}\left(q_{1}(t)\right), N_{2}^{\prime \prime}\left(q_{2}(t)\right)=0$ folgt 2.41 . 
mit $\Omega=2 N_{1}{ }^{\prime}\left(q_{1}(t)\right) N_{2}{ }^{\prime}\left(q_{2}(t)\right)-C^{\prime \prime}(q(t))\left[N_{1}{ }^{\prime}\left(q_{1}(t)\right)+N_{2}{ }^{\prime}\left(q_{2}(t)\right)\right]>0$.

Da für Markt 1 eın unelastischerer Nachfrageverlauf als für Markt 2 unterstellt wurde, zeıgt die Entwicklung der gleichgewichtigen Handelsmengen des Marktes 1 einen flacheren Verlauf als die des Marktes 2:

$$
\left.N_{1}{ }^{\prime}\left(q_{1} / t\right)\right)<N_{2}{ }^{\prime}\left(q_{2}(t)\right)<0 \Rightarrow d q_{2} / d t<d q_{1} / d t<0 .
$$

Die Entwicklung der gesamten Abbaumenge folgt aus $d q / d t=d q_{1} / d t+d q_{2} / d t$ mit 2.42:

$$
d q / d t=\delta \lambda e^{\delta t}\left[N_{1}{ }^{\prime}\left(q_{1}(t)\right)+N_{2}{ }^{\prime}\left(q_{2}(t)\right)\right] \Omega^{-1}(t)<0 .
$$

Im Zeitraum [0, $T_{1}$ ] bietet der Monopolist auf beiden Teilmärkten seine Rohstoffe an. Da $N_{2}(0)<N_{1}(0)$ unterstellt wurde, wird für $d p_{1} / d t=d p_{2} / d t d e r$ "choke-off" Preis des zweiten Teilmarktes zuerst erreicht sein. Dies geschieht in $T_{1}$. Bis zur Erschöpfung des Ressourcenbestandes oder der Gleichheit von Durchschnitts- und Grenzgewinn aus der Abbautätigkeit beliefert der Monopolist dann nur noch den ersten Teilmarkt (Schaubild:2.2)

$$
q(t)=\left\{\begin{array}{lll}
q_{1}(t)+q_{2}(t) & t \in\left[0, T_{1}\right] \\
q_{1}(t) & \text { für } & t \in\left(T_{1}, T\right]
\end{array}\right.
$$

Da $d p_{1} / d t=d p_{2} / d t$, ermittelt man für die Relation der Rohstoffpreise zu Beginn der Abbautätigkeit

$$
p_{1}(0)><p_{2}(0) \Leftrightarrow p_{1}\left(T_{1}\right)><N_{2}(0) .
$$

Das Abbauprofil nach 2.43 wird auch realisiert, wenn der Monopolist eine Preisdifferenzierung nicht vornimmt. Denn für die Gesamtnachfrage $N^{-1}(p(t))$ würde gelten

$$
\begin{aligned}
& N^{-1}(p(t))=N_{1}{ }^{-1}(p(t))+N_{2}{ }^{-1}(p(t)) \text { mit } \\
& N^{\prime}(q(t))=N_{1}{ }^{\prime}\left(q_{1}(t)\right) N_{2}{ }^{\prime}\left(q_{2}(t)\right)\left[N_{1}{ }^{\prime}\left(q_{1}(t)\right)+N_{2}{ }^{\prime}\left(q_{2}(t)\right)\right]^{-1}
\end{aligned}
$$


Schaubild 2.2: Die intertemporalen Handelsprofile bei Preisdifferenzierung unter Sicherheit
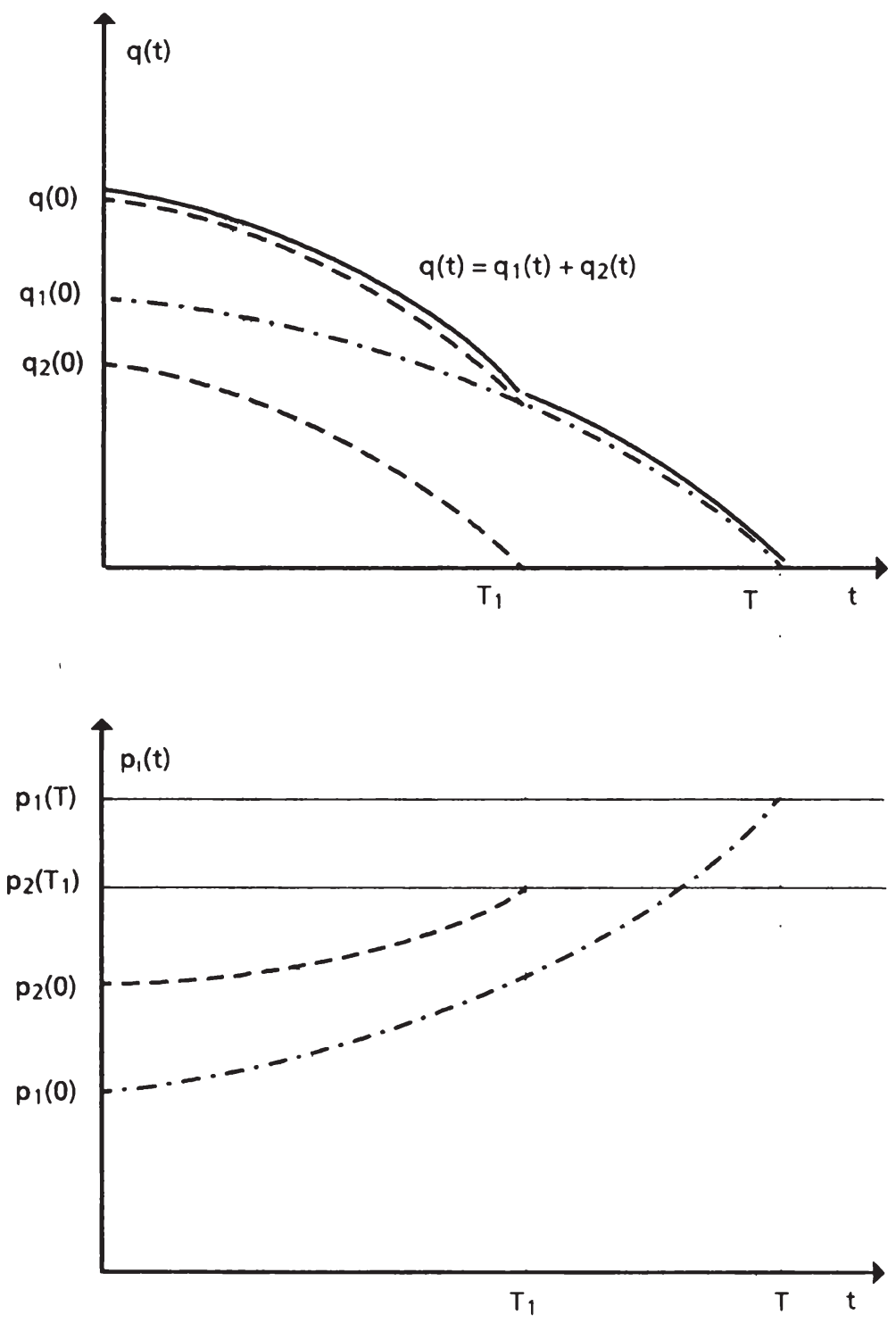
und die notwendige Bedingung für gewinnmaximales Angebot würde liefern

$$
d q / d t=\delta \lambda e^{\delta t}\left[2 N^{\prime}(q(t))-C^{\prime \prime}(q(t))\right]-1<0 .
$$

Die Abbauprogramme des Monopolisten sind mit und ohne Preisdifferenzierung identisch. 


\subsection{Das Oligopol: Mengenkonkurrenz im homogenen Duopol}

Charakteristisch für die Marktform des Oligopols, speziell des Duopols, wie es später betrachtet werden soll, ist die Tatsache, daß jeder Anbieter den Marktpreis beeinflussen, aber nicht vollständig kontrollieren kann. Der Gewinn des eınzelnen Abbauunternehmers ist abhängig von seinen Konkurrenten. Sein Angebotsverhalten wird somit beeinflußt von den Erwartungen, die er über das Verhalten seiner Konkurrenten hat.

Als Lösungskonzepte bietet die Theorie der Differentialspiele open-loop oder closed-loop Nash-Gleichgewichte an. Die Nash-Lösung eines Duopols fordert, daß jeder Anbieter das Abbauprogramm wählt, bei dem er bei gegebenem Abbauprogramm des Konkurrenten seinen Gewinn maximiert. Bei einer open-loop-Lösung sind die Abbauentscheidungen des Unternehmers zu einem bestimmten Zeitpunkt unabhängig von vorangegangenen Entscheidungen. Der Abbauunternehmer plant zu Beginn der Abbautatigkeit sein gesamtes Abbauprogramm. Die closed-loop-Lösung dagegen unterstellt einen mehrstufigen Entscheidungsprozeß. Der Abbauunternehmer plant zu jedem Zeitpunkt seiner Abbautätigkeit neu. Er maximiert zu jedem Zeitpunkt neu den gesamten Barwert der Periodengewinne des verbleibenden Abbauzeitraumes.

Die einzigen Autoren, die bisher closed-loop-Lösungen für eine symmetrische oligopolistische Angebotsstruktur in der Ressourcentheorie vorgelegt haben, sind Gottwald und Güth. 1)

Ebenfalls eine symmetrische Angebotsstruktur (Cournot-Lösung) wird in den meisten Beiträgen zu open-loop-Lösungen betrachtet: Salant (1976. 1982), Lewis/Schmalensee (1978a), Hoel (1978), Loury (1980) und Ulph/Folie (1980) sind hier zu nennen.

1) Gottwald/Guth (1980) und Gottwald (1981). 
Eine asymmetrische Angebotsstruktur bel open-loop Losungen (Stackelberg-Lösung) wurde von Cremer,Weitzman (1976), Gilbert (1978), Hoel (1978), Salant (1979) und Lewis/Schmalensee (1980b) diskutiert. Hier gibt das domınante Förderunternehmen seıne Mengen- und Preısplanungen unter Berúcksichtıgung der Reaktıonen der abhängigen Konkurrenten bekannt. Die Förderunternehmen in der Abhängıgkeitspositıon passen sich mit ihren Abbauprogrammen an die Entscheidungen des Unternehmens in der Unabhängıgkeıtsposition an.

Beide Gleıchgewichtskonzepte, die der Cournot- und die der StackelbergLösung, können zu demselben gleichgewichtigen Preispfad führen. 1) Der Stackelberg-Ansatz zeigt jedoch im Vergleich mit dem Cournot-Ansatz die nicht wünschenswerte Eigenschaft der dynamischen Inkonsistenz. 2) Im folgenden wird deshalb die Symmetrieannahme Cournots gemacht.

Die einfachste Form des Oligopols ist das Duopol. "Alle wesentlichen Merkmale der oligopolistischen Marktform sind bereits im Duopol angelegt und dort, ..., am leichtesten abzuleiten. "3) Auf die Betrachtung oligopolistischer Angebotsstrukturen mit mehr als zwei Anbietern soll darum in dieser Arbeit nicht eingegangen werden.

Weiter kann für die Analyse unterstellt werden, daß die Rohstoffe homogen in den Augen der nachfragenden Verarbeitungsunternehmen oder der Konsumenten sind.

Von den beiden Möglichkeiten, die homogene Duopolsituation unter Mengen-oder Preiskonkurrenz zu betrachten, soll die des mengenstrategischen Verhaltens gewählt werden.4) Dieser Duopoltyp ist nicht nur leichter zu

\footnotetext{
1) Dies hat Gilbert (1978) gezeigt.

2) Hierzu siehe Kydland/Prescott (1977), Ulph (1980). Dynamısch ınkonsistent meint, daß der Preispfad abhangıg ist vom Anfangszeitpunkt der Planung.

3) Krelle (1976), S.131.

4) Zu eınem preisstrategischen Verhalten in einer homogenen Duopolsituation siehe Hoel (1978), S.39-54.
} 
analysieren, sondern er erlaubt auch den direkten Vergleich mit den vorangehenden Modellergebnissen.

Für die Duopolisten sollen die folgenden Annahmen gelten:

Jede Unternehmung baut einen bekannten Ressourcenbestand $R_{01}$ mit konstanten Durchschnittskosten $c_{i}>0$ ab. 1)

$q(t)=q_{1}(t)+q_{2}(t)$ ist die gesamte Abbaumenge in der Periode $t$ und stellt das Angebot am Rohstoffmarkt dar. Beide Duopolisten kalkulieren mit der gleichen Diskontrate. Die polypolistisch strukturierte Nachfrageseite sei wieder repräsentiert durch die Funktion

$$
p(t)=N(q(t)), \text { mit } N^{\prime}(q(t))<0, N^{\prime \prime}(q(t))=0 .
$$

Bei beiden Duopolisten wird autonomes Verhalten unterstellt, d.h. jeder Duopolist vermutet vom anderen, daß dieser an seiner bisherigen Angebotsmenge festhält, unabhängig davon, was er selbst tut. Jeder Duopolist wählt das Abbauprogramm, bei dem er für ein gegebenes Abbauprogramm des Konkurrenten den Gegenwartswert seiner gesamten Gewinne unter Beachtung der Ressourcenrestriktion maximiert. Kein Duopolist vermutet, daß er seinen optimalen Gewinn durch ein anderes als das geplante Verhalten verbessern kann (Nash-Gleichgewicht).

Die Duopolisten kooperieren nicht. Eine gemeinsame Gewinnmaximierung würde zu dem Allokationsergebnis eines Monopols führen.

Beiden Duopolisten stellt sich dann das Optimierungsproblem, $i=1,2$ :

$$
\begin{aligned}
& \max _{\left(q_{1}(t), T_{i}\right)}{ }_{0} T^{T_{1}}\left[p(t) q_{1}(t)-c_{1} q_{1}(t)\right] e^{-\delta t d t} \\
& \text { u.d.B. } \quad R_{0_{1}} \geq{ }_{0} \int^{T_{1}} q_{1}(t) d t, R_{0 i} \text { gegeben, } q_{1}(t) \geq 0, p(t)=N(q(t)) .
\end{aligned}
$$

1) Die Annahme konstanter Durchschnittskosten erleichtert hier die Analyse. 
An notwendigen Bedingungen erhalt man für den i-ten Anbieter 1):

$(2.45)(1) \quad\left[p(t)+N^{\prime}\left(q_{1}(t)\right) q_{1}(t)-c_{1}\right] e^{-\delta t}-\lambda_{i}$

$$
\begin{aligned}
& \begin{cases}=0 & q_{1}(t)>0 \\
\text { furr } & \\
\leq 0, & q_{1}(t)=0\end{cases} \\
& \left\{\begin{array}{lll}
=0 & & \lambda_{1}>0 \\
& \text { für } & \\
\geq 0 & & \lambda_{1}=0
\end{array}\right.
\end{aligned}
$$

(2.45)(ii) $\quad R_{01}-{ }_{0} \int^{T_{1}} q_{1}(t) d t$

$(2.45)$ (iii) $\left[p\left(T_{1}\right)-c_{1}\right] q_{1}\left(T_{1}\right) e^{-\delta T_{1}-\lambda_{i} q_{i}\left(T_{1}\right)=0}$

Sei $n(t)$ die Preiselastizität der Nachfrage und $\alpha_{1}(t)=q_{1}(t) / q(t)$ der Marktanteıl des i-ten Duopolisten, so folgt in der äquıvalenten Schreibweise zu 2.30 für positive Angebotsmengen $q_{1}(t), q_{2}(t)>0$

$$
p(t)\left[1+\alpha_{1}(t) / n(t)\right]-c_{i}=\lambda_{1} e^{\delta t}, i=1,2
$$

Die Duopolisten bauen wie die Anbieter in anderen Marktformen mit den Mengen ab, die ihnen in Gegenwartswerten einen für alle Perioden konstanten Grenzgewinn liefern. Die Gegenwartswerte der Grenzgewinne beider Duopolisten sind im allgemeinen verschieden, $\lambda_{1}><\lambda_{2}$.

Für die Abbauprofile läßt sich aus 2.45(i) ermitteln

$(2.47)(1) \quad d q_{1} / d t=\delta e^{j t}\left[2 \lambda_{1}-\lambda_{2}\right]\left[3 N^{\prime}(q(t))\right]^{-1}$

$(2.47)$ (ii) $\quad d q_{2} / d t=i e^{o ̈ t}\left[2 \lambda_{2} \cdot \lambda_{1}\right]\left[3 N^{\prime}(q(t))\right]^{-1}$.

so daß für die zeıtliche Entwicklung der Handelsmengen des Marktes und damit fur die Nutzung des gesamten Ressourcenbestandes $R_{0}=R_{01}+R_{02}$

1) Zu den Ergebnissen in (i), (ii) vgl. die äquivalenten Ergebnisse bei Dasgupta/ Heal (1979), S.338 oder Lewis/Schmalensee (1980), S.478 
folgt

(2.48) $\quad d q / d t=\delta e^{o t}\left[\lambda_{1}+\lambda_{2}\right]\left[3 N^{\prime}(q(t))\right]^{-1}<0$.

Der gleichgewichtige Preispfad folgt der Entwicklung

$$
d p / d t=(1 / 3) \delta e^{\delta t}\left[\lambda_{1}+\lambda_{2}\right]>0 .
$$

Für den Vergleich der Duopolsituation mit den Marktformen des Monopols und des Polypols und zur Ermittlung weiterer Aussagen 1) seien für die Duopolisten gleiche Durchschnittskosten des Abbaus unterstellt, $c_{1}=c_{2}=c$.

Aus den Gleichungen 2.5, 2.6 für das Polypol und 2.31, 2.32 für das Monopol werden unter der Annahme konstanter durchschnittlıcher Abbaukosten die folgenden Mengen- und Preispfade erhalten. P, D, M kennzeichnen als Indices die Entwicklungspfade unter den Markformen des Polypols, des Duopols und des Monopols:

(2.50)(i) $(d q / d t)_{p}=\delta[p(t)-c] N^{\prime}(q(t))-1<0$

(2.50)(ii) $\quad(d p / d t) p=\delta[p(t)-c]>0$

(2.51)(i) $(d q / d t)_{D}=(2 / 3) \delta\left[p(t)-c+N^{\prime}(q(t)) q(t) / 2\right] N^{\prime}(q(t))-1<0$

(2.51)(ii) $(d p / d t)_{D}=(2 / 3) \delta\left[p(t)-c+N^{\prime}(q(t)) q(t) / 2\right]>0$

(2.52)(i) $(d q / d t)_{M}=(1 / 2) \delta\left[p(t)-c+N^{\prime}(q(t)) q(t)\right] N^{\prime}(q(t))-1<0$

(2.52)(ii) $\quad(d p / d t) M=(1 / 2) \delta\left[p(t)-c+N^{\prime}(q(t)) q(t)\right]>0$.

Hieraus folgt $\quad(d q / d t)_{p}<(d q / d t)_{D}<(d q / d t)_{M}<0$ und

$$
(d p / d t)_{P}>(d p / d t)_{D}>(d p / d t)_{M}>0 \text {. }
$$

1) Läßt man ungleiche Abbaukosten zu, dann sind schon in diesem Fall eindeutige Aussagen nicht mehr möglich. Siehe hierzu Lewis/Schmalensee (1980), S.485f. 
Ein bestimmter, gegebener Ressourcenbestand wird unter den getroffenen Annahmen von einem Monopolisten langsamer abgebaut als von Duopolisten, und diese bauen ihn wiederum langsamer ab als Polypolisten. Die konservierende Wirkung des Duopols ist geringer als die des Monopols.

Aus den notwendigen Bedingungen 2.45 (i) folgt weiter für $c_{1}=c_{2}=c$ die Aussage, daß ein höherer Schattenpreis (Gegenwartswert des Grenzgewinnes) geringere Abbaumengen zu jedem Zeitpunkt induziert, was zu elner fruheren Erschöpfung des Ressourcenbestandes führt und einen kleineren Ressourcenbestand folgern läßt. Aus

$$
N^{\prime}(q(t))\left[q_{1}(t)-q_{2}(t)\right]=e^{o i t}\left[\lambda_{1}-\lambda_{2}\right]
$$

folgt wegen $N^{\prime}(q(t))<0$

$$
\left.\lambda_{1}><\lambda_{2} \Leftrightarrow q_{1}(t)><q_{2}(t) \Leftrightarrow T_{1}><T_{2} \Leftrightarrow R_{01}><R_{02} .1\right)
$$

Dıes ist konsistent mit der Transversalitätsbedingung 2.45 (ii), die für den Zeitpunkt $T_{\text {, liefert }} \mathrm{q}_{i}\left(T_{1}\right)=0$.

Sei $R_{01}<R_{02}$ gegeben, dann folgt $\lambda_{1}>\lambda_{2}$ mit $q_{1}(0)<q_{2}(0)$, d.h. der Grenzerlös des Duopolisten 1 mit dem geringeren Reservenbestand wächst auf einem höheren Niveau. 2.47 liefert mit 2.45 (i) die Ungleichung (dq/dt) $1<$ $(\mathrm{dq} / \mathrm{dt})_{2}<0$.2) Duopolist 2 baut mit weniger stark sinkenden Fördermengen ab als Duopolist 1. In $T_{1}$ ist der Bestand des Duopolisten 1 erschöpft. Duopolist 2 bietet allein auf dem Markt an bis in $T_{2}$ auch sein Reservenbestand erschöpft ist.

1) Dieses Ergebnis leiten auch Lewis/Schmalensee (1980), S.481 ab.

2) Es gilt: $\mathrm{dq}_{1} / \mathrm{dt}<\mathrm{dq}_{2} / \mathrm{dt}<0 \Leftrightarrow \lambda_{1}>\lambda_{2}$. 
Schaubild 2.3: Die intertemporale Ressourcenallokation bei verschiedenen Marktformen unter Sicherheit
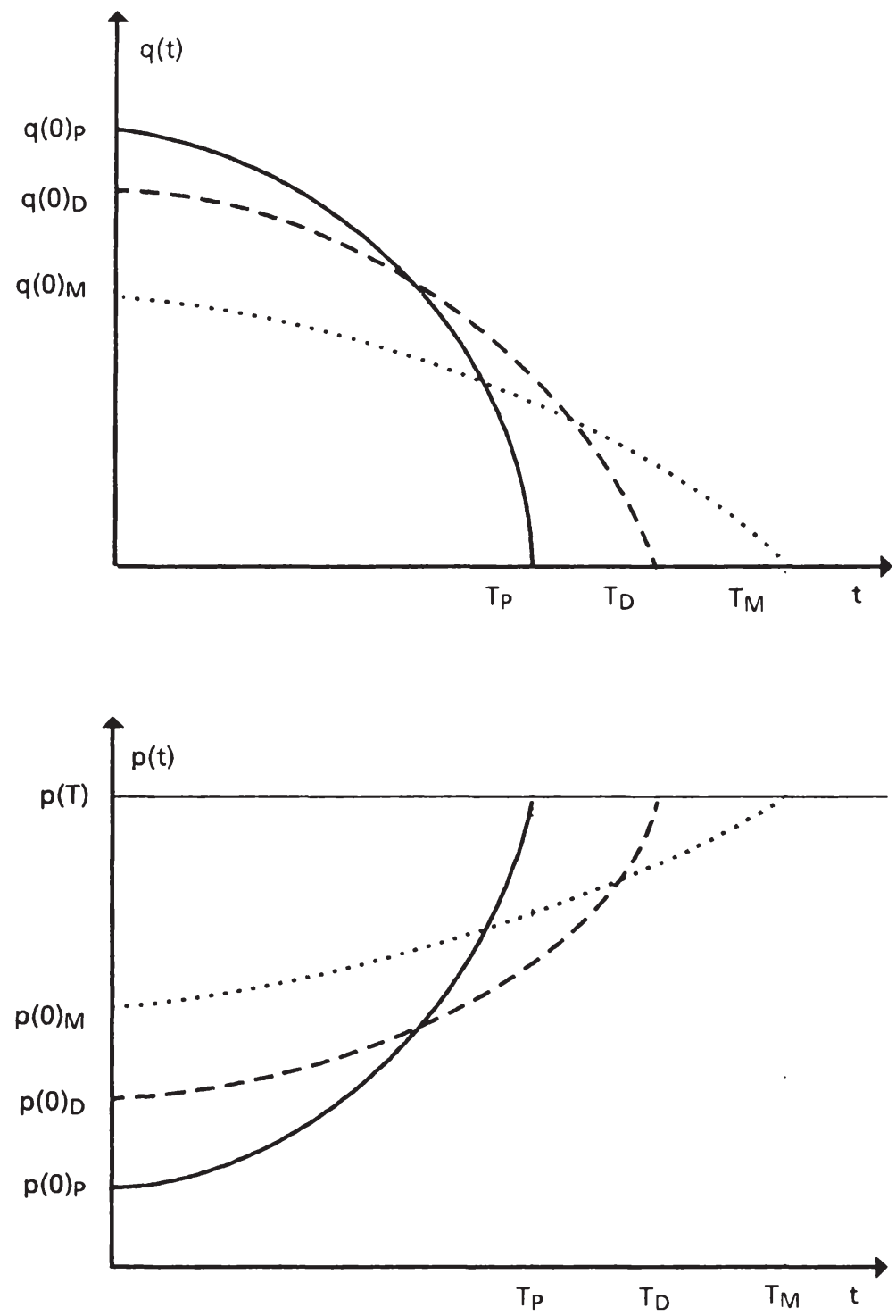


\subsection{Ein zweistufiges Marktkonzept zur theoretischen Interpretation der Funktionsweise von Rohstoff-und Reservenmarkt}

Dieser Abschnitt dient zur Vorbereitung der Darstellung !n Abschnitt 6.2., wo unter Risıko auf dem Rohstoffmarkt Vertragsverembarungen zwischen Abbauunternehmern und Reservenbesitzern diskutıert werden.

Betrachtet werden dreı Arten von Wirtschaftssubjekten: Reservenbesitzer, Abbauunternehmer und Verarbeitungsunternehmer. Zwischen diesen Wirtschaftssubjekten existieren für den Ressourcenhandel zwei Märkte: ein Reservenmarkt und eın Rohstoffmarkt. 1) Beıde Markte seien vollkommene Konkurrenzmärkte. Sowohl der Rohstoff-als auch der Reservenmarkt bestehen jeweils aus einem Kassamarkt und T Termınmärkten, wenn die Abbautatigkeit $T+1$ Perioden andauert.

Auf dem Rohstoffmarkt seien die Verarbeitungsunternehmen repräsentiert durch die lineare, zeıtlich invarıante Nachfragefunktion 2.4.

Die Förderunternehmen bauen mit konstanten Durchschnittskosten c den Rohstoff aus den Lagerstatten ab. Zu diesem Zweck fragen sie Reserven in Form von Abbaurechten bei den Reservenbesitzern nach. Für gegebene Folgen von Preisen für Abbaurechte pro Mengeneinheit der Reserve $\{r(t)\}$ und Preisen fur Rohstoffe $\{p(t)\}$ wahlen die Abbauunternehmer ihre Nachfrage nach Abbaurechten und ihr Angebot an Rohstoffen so, daß sie die Gesamtheit der diskontierten Periodengewinne maximieren:

1) Zum Konzept eines Reservenmarktes siehe Howe (1979), S.98-101. 
(2.54) $\quad \max { }_{0} \int^{T}[p(t)-c-r(t)] q(t) e^{-\delta} t d t$

$(q(t))$

u.d.B. $q(t) \geq 0$.

Alle Reservenbesitzer kennen ihre Lagerberstände $R_{0}$. Sie bieten ihre Reserven nur in Form von Abbaurechten an und bauen selbst keıne Rohstoffe ab. Als Zielfunktion maxımieren sie ebenfalls die Gesamtheit aller diskontierten Perrodengewinne:

(2.55) $\quad \max { }_{0} \int^{T} r(t) q(t) e-\delta t d t$ $(q(t))$

u.d.B. $R_{0} \geq{ }_{0} f^{\top} q(t) d t, R_{0}$ gegeben, $q(t) \geq 0$.

Neben dem Reserven- und Rohstoffmarkt existiere ein vollkommener Kapitalmarkt, auf dem die Gewinne mit der risikolosen Zinsrate $\delta$ angelegt werdenkönnen.

Aus den Optimierungskalkülen 2.54 und 2.55 können dann die Nachfrage und das Angebot an Abbaurechten abgeleitet werden.

$$
r(t)=p(t)-c \text {, für } q(t)>0 \text { aus } 2.54 \text { und }
$$

$$
r(t)=\lambda e^{\delta t}, \quad \text { für } q(t)>0 \text { aus } 2.55
$$

Im Gleichgewicht auf Rohstoff- und Reservenmarkt gilt dann die Bezıehung

$$
N(q(t))=r_{0} e^{\delta t}+c .
$$

Für die zeitliche Entwicklung der Abbaumengen und der Preise gilt

$$
\mathrm{dq} / \mathrm{dt}=\delta r_{0} e^{\delta t} \mathrm{~N}^{\prime}(\mathrm{q}(\mathrm{t}))^{-1}<0 \text { und }
$$




$$
d p / d t=d r / d t=\delta r(t)>0 .
$$

Dies sind die bekannten Ergebnisse aus den Grundmodellen der Ressourcentheorie. Der Unterschied besteht nun in der Interpretation des Zustandekommens der gleichgewichtıgen Preis- und Mengenpfade für die intertemporale Ressourcenallokation.

Bevor die Rohstoffanbieter mit dem Abbau beginnen, treten sie auf Reserven- und Rohstoffmarkt den Reservenanbietern und Rohstoffnachfragern gegenüber. Sie erhalten für jede Periode Zusagen über die Nutzung bestımmter Reservenmengen und machen Zusagen über die Lieferung von Rohstoffen für jede Periode unter dem Vorbehalt der Rücknahme der Zusage für den Fall, daß Nutzungsrechte (Abbaurechte) für Reserven zu einem geringeren Preis erhalten, bzw. Rohstoffe zu einem höheren Preis verkauft werden können. Dieselbe Vorbehaltszusage steht auch dem jeweiligen Handelspartner der Marktgegenseite zu.

Kommt in einem ersten Abschluß ein Rohstoffpreis $p_{1}(t)$ (Reservenpreis $\left.r_{1}(t)\right)$ zustande, dann werden alle folgenden Abschlüsse zu demselben Preis getätigt. Erhalten nicht alle potentiellen Rohstoffnachfrager (Reservenanbieter) Zusagen über Rohstofflieferungen (Reservenabnahmen) und übersteigt (unterschreitet) deren Zahlungsbereitschaft (Preisforderung) den bisherigen Preis $p_{1}(t)\left(r_{1}(t)\right)$, dann werden diese versuchen, zu einem Preis $p_{2}(t)>p_{1}(t)\left(r_{2}(t)<r_{1}(t)\right)$, Abschlüsse zu erhalten. Bei diesen Preisen werden die bisherigen Abschlüsse von den Abbauunternehmern zurückgenommen, um nun zu dem höheren Preis $p_{2}(t)$ (niederen Preis $r_{2}(t)$ ) mit anderen Rohstoffnachfragern (Reservenanbietern) abschließen zu können. Einige Nachfrager (Anbieter) werden keine neuen Abschlüsse bzw. Abschlüsse mit geringeren Mengenzusagen tätigen. Schließlich wird ein Preis $p(t)_{g}\left(r(t)_{g}\right)$ erreicht werden, bei dem sich nachgefragte und angebotene Rohstoffmenge (angebotene und nachgefragte Reservenmenge) dieses Zeitpunktes entsprechen.

Solch ein Prozeß des "verbalen Recontracting" läuft nun nicht getrennt für jeden Zeitpunkt ab - also getrennt für jeden Zukunftsmarkt -, sondern es 
finden auch Zurücknahmen von Zusagen statt aufgrund von Differenzen zwischen den diskontierten Preisen zweier Perıoden

$$
d p / d t><\delta r(t) \quad(b z w . d r / d t><\delta r(t)) .
$$

Für ein Preisangebot $d p / d t>\delta r(t)(d r / d t>\delta r(t))$ werden die Abbauunternehmer (Reservenbesitzer) Lieferzusagen (Nutzungsrechte) für den Zeitpunkt $t$ zurückziehen und für einen späteren Zeitpunkt tätigen. Erst wenn für die Gleichgewichtspreise der verschiedenen Perioden

$$
d p_{g} / d t=\delta r_{g}(t) \quad\left(b z w . d r_{g} / d t=\delta r_{g}(t)\right) \text { gilt, }
$$

haben weder Rohstoffnach frager noch Abbaunternehmer noch Reservenbesitzer einen Anreiz, nach neuen Abschlüssen zu suchen.

Zwischen den temporären Gleichgewichtswerten der beiden Märkte gilt dann die Beziehung

$$
p_{g}(t)=r_{g}(t)+c
$$

Denn bei der Ungleichung $p(t)>r(t)+c$ würden die Abbauunternehmer weiter um Abbaurechte und im Angebot weiter mit Rohstoffen konkurrieren, bis der durch Nachfragekonkurrenz steigende Preis für die Abbaurechte und der durch Angebotskonkurrenz fallende Preis für Rohstoffe die Gleichheit hergestellt hat.

Eine graphische Betrachtungsweise des beschriebenen zweistufigen Marktsystems ist in einem Vierquadrantenschema möglich. 1)

Schaubild 2.4 stellt in Quadrant I den Rohstoffmarkt dar, in Quadrant II den Reservenmarkt, in Quadrant III die Bestandsrestriktion der Reserven und in Quadrant IV die Transformation der Rohstoff-in Reserveneinheiten.

1) Vgl. hierzu Howe (1979), S.99, in anderer Darstellungsweise. 
Schaubild 2.4. Reserven- und Rohstoffmarkt für zwel aufeinanderfolgende Zeitpunkte $t$ und $t+1$

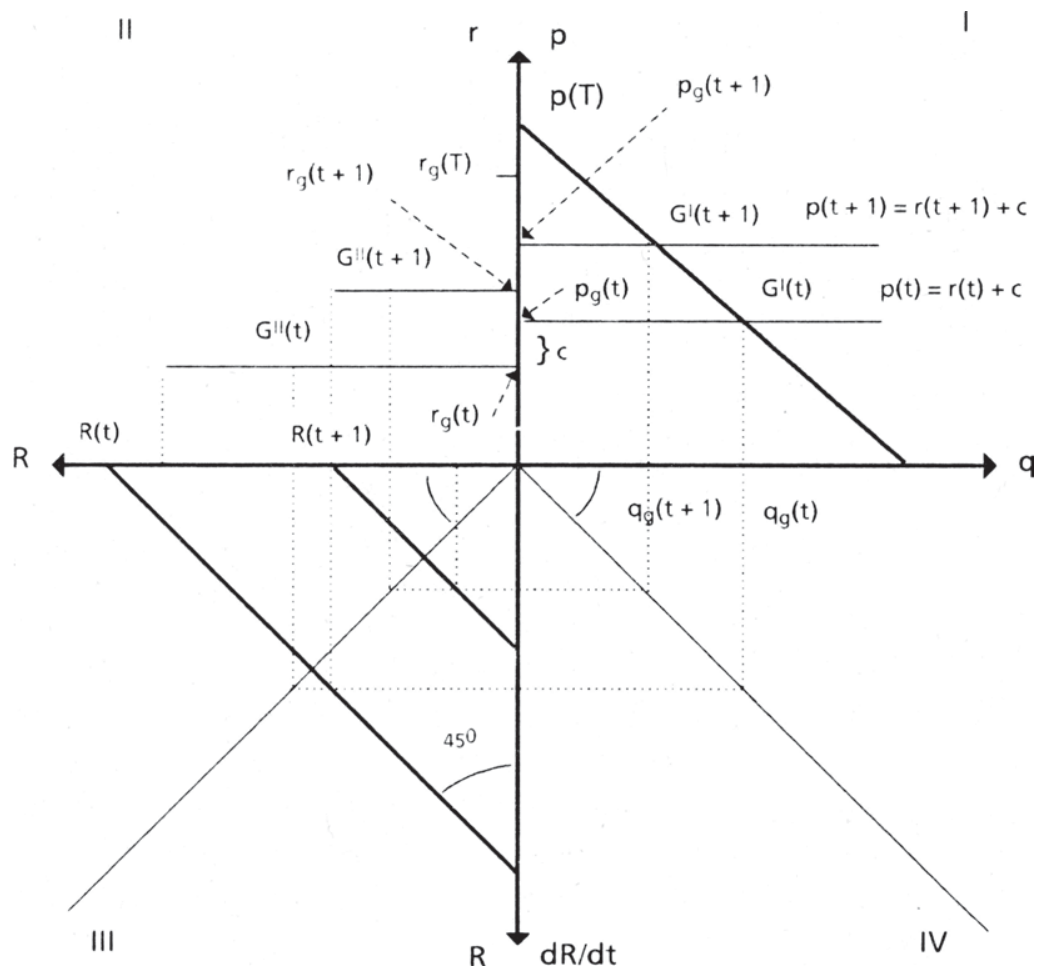

Das Schaubild stellt fur die Zeitpunkte $t$ und $t+1$ die Abfolge der temporären Gleichgewichte auf dem Reservenmarkt (GII(t), GII $(t+1)$ ) und dem Rohstoffmarkt $\left(G^{\prime}(t), G^{\prime}(t+1)\right)$ dar:

Wächst der Reservenpreis (der Preis für Abbaurechte) mit der Rate $\delta$, dann sind die Reservenbesitzer zu jedem Zeitpunkt indifferent zwischen dem Verkauf und dem Nichtverkauf ihrer Reservenbestände. Das Angebot an Abbaurechten ist zum Preis $r_{g}(t)$ unendlich elastisch im Intervall $[0, R(t)]$. 
Welche Reservenmenge über Abbaurechte in der Periode $t$ zum Abbau genutzt wird , bestımmt die aus der Rohstoffnachfrage der Verarbeitungsunternehmen abgeleitete Reservennach frage der Abbauunternehmen. Diese ist unter den gemachten Annahmen ebenfalls unendlich elastisch in $r_{g}(t)$.

Hat sich auf dem Rohstoffmarkt die Situation $G^{\prime}(t)$ eingestellt, dann bestimmt die Rohstoffmenge $q_{g}(t)$ in Quadrant IV die zum Abbau erforderliche Reservenmenge. 1)

In Quadrant III überträgt die Winkelhalbierende die Reservenmenge $\mathrm{dR} / \mathrm{dt}$ von IV nach II und bestimmt in Quadrant II das Gleichgewicht GII $(t)$ auf dem Reservenmarkt. Die Gerade von $R(t)$ nach $R(t)$ im III. Quadranten steht für die Ressourcenrestriktion. Überträgt man mit ihr die Reservennachfrage der Periode $t$ von IV nach II, so erhält man im II. Quadranten den Reservenbestand der Periode $t+1$, also das Angebot an Abbaurechten für die Folgeperiode.

Zeigte Schaubild 2.4 die Abfolge zweier aufeinanderfolgender Gleichgewichte der Perioden $t$ und $t+1$, so wird in Schaubild 2.5 die Anfangssituation (GI(0), GII(0)) der Endsituation (GI(T), GII(T)) gegenübergestellt. Ausgehend von einem Reservenbestand $R_{0}$ und der $z u t=0$ gehörenden Gleichgewichtssituation (GI(0), GII(0)) liefert der Reservenmarkt für die intertemporale Ressourcenallokation eine Preisentwicklung $r_{g}(t)=r_{0} e^{\delta t}$. Die Rate $\delta$ bestimmt über die Angebots- und Nachfragefunktion des Rohstoffmarktes auch die Entwicklung der Rohstoffpreise und Rohstoffmengen.

Unterstellt man zur Ermittlung der Preis- und Mengenpfade wie in den Schaubildern eine Rohstoffnachfrage der $\operatorname{Art} p(t)=p(T)-b q(t)$, so erhält man:

1) Für den Abbau einer Rohstoffeınheit sei genau eine Reserveneınheit erforderlich. 
Schaubild 2.5: Reserven- und Rohstoffmarkt zu Beginn und am Ende der Abbautätıgkeit

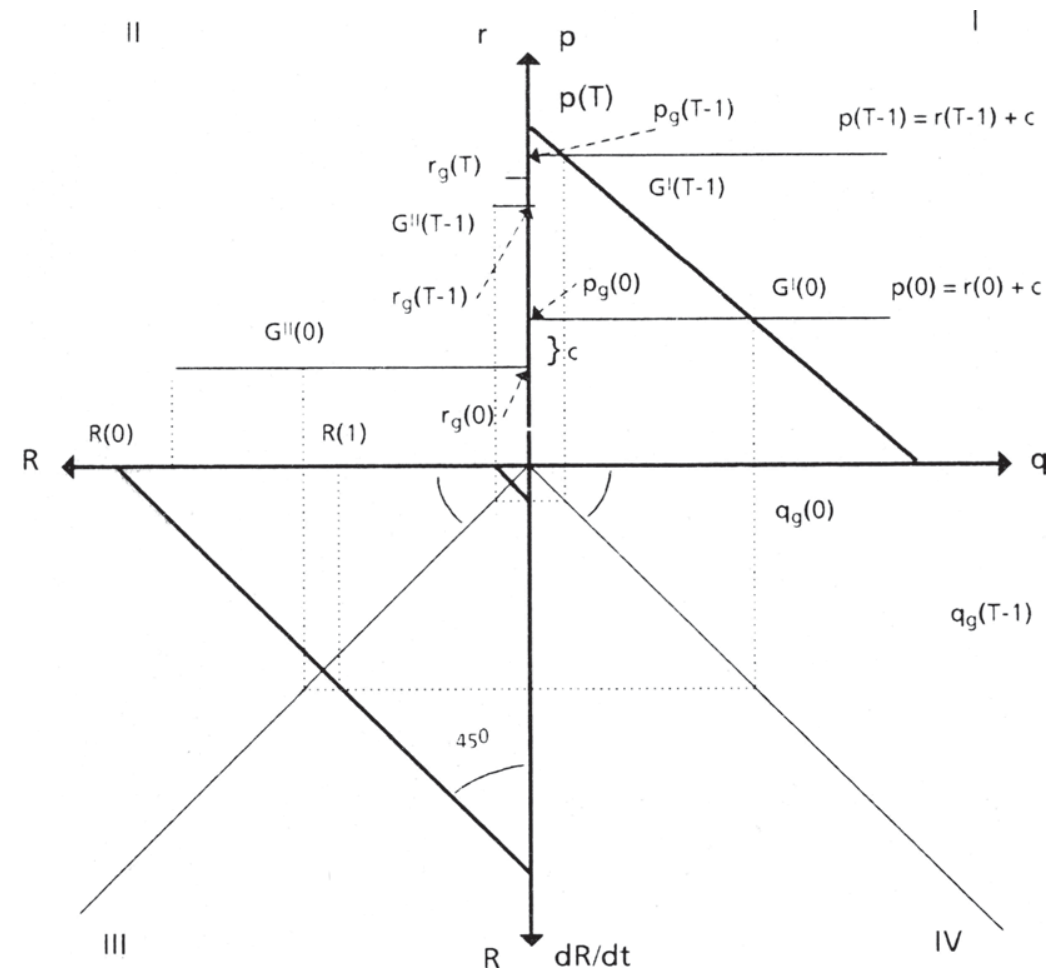

(2.61) $\quad p_{g}(t)=r_{0} e^{\delta t}+c$

(2.62) $\quad q_{g}(t)=\left[p(T)-r_{0} e^{\delta t}-c\right] b^{-1}$,

mit $r_{0}=\left[p(T)-c-b R_{0} T-1\right] \delta T\left(e^{\delta T}-1\right)^{-1}$.

$r_{0}$ bestimmt sich dabei so, daß im Zeitpunkt T zum Preıs $p(T)$ mit der Entnahme der Menge $q(T-1)$ der Reservenbestand $R(T)$ Null wird. In $T+1$ sind dann sowohl die Rohstoffnachfrage als auch das Rohstoffangebot und damit auch die Reservennachfrage auf Null gesunken. 
Zusammen mit den temporären Stromgleichgewichten jeder Periode besteht auch ein ıntertemporales Bestandsgleichgewicht über alle Perioden.

Auf eine exogene Variation der Diskontrate $(d \delta)$, des Ressourcenbestandes $\left(d R_{0}\right)$, der Abbaukosten ( $\left.d c\right)$ und der Nachfrage $(d p(T), d b)$ paßt sich das System über neue Werte für den Abbauzeitraum und den Reservenpreis ro wie folgt an:1)

Tabelle 2.1: Ergebnisse einer komparativen Analyse

\begin{tabular}{l|ccccc} 
& $\delta$ & $R_{0}$ & $c$ & $p(T)$ & $b$ \\
\hline$T$ & - & + & $+2)$ & $-2)$ & + \\
$r_{0}$ & - & - & - & + & -
\end{tabular}

Mit abnehmender Diskontrate, zunehmendem Ressourenbestand und zunehmenden Durchschnittskosten des Abbaus wird der Abbauzeitraum größer. Der Reservenpreis sinkt, wenn die Diskontrate, der Ressourcenbestand und die durchschnittlichen Abbaukosten zunehmen.

1) Siehe Anhang I. Die Elemente der Tabelle geben das Vorzeichen des Differentialquotıenten aus endogener und exogener Variablen an, bspw. dT/d $\delta<0$. Zu einer ahnlichen komparatıven Analyse siehe Hartwick (1978). Siebert (1983), S.71-75 diskutiert graphisch-verbal einıge Parameteränderungen.

2) Für $\delta T>1$. 
Steigt die Diskontrate im System, dann führt dies dazu, daß zukünftige Erträge gegenüber heutıgen Erträgen stärker diskrıminiert werden. Gegenwartsnähere Erträge werden gegenwartsferneren Erträgen vorgezogen. Das Angebot an Reserven und der Abbau der Lagerstätten wird in die Gegenwart verlagert. Bei unveränderter Nachfrage werden die Gleichgewichtspreise der Rohstoffe und wegen konstanter durchschnittlicher Abbaukosten auch die der Reserven sinken. 1) Der Reserven preis wächst mit der größeren Diskontrate. Die Fördermengen nehmen mit größeren Veränderungen $a b$. Bei gegebenem Reservenbestand sind die Läger früher erschöpft.

Eine Vergrößerung des Reservenbestandes führt aufgrund der unverảnderten Nachfragesituation des Rohstoffmarktes ebenfalls zu sinkenden Rohstoff- und Reservenpreisen. Da nun jedoch der Diskontsatz und damit die zeitliche Entwicklung der Abbaumengen unverändert geblieben ist, erhöhen die Abbauunternehmer den Abbauzeitraum, indem sie mit dem Abbau beı einem kleineren Marktpreis für Rohstoffe beginnen.

Eine exogene Steigerung der durchschnittlichen Abbaukosten verringert das Angebot der Abbauunternehmer auf dem Rohstoffmarkt und damit auch deren Nachfrage auf dem Reservenmarkt. Die Rohstoffpreise werden steigen, der Gegenwartswert des Reservenpreises wird sinken. Da die Rohstoffpreise nun mit geringeren Veränderungen steigen, dehnen die Abbauunternehmer den Zeitraum ihrer Fördertätigkeit aus.

Eine Änderung der Nachfrage hat unterschiedliche Auswirkungen auf die Ressourcenallokation. Erhöht sich der choke-off Preis der Nachfrage, so

1) Wenn hier von sınkenden/steıgenden Preısen die Rede ıst, so bezıeht sich dies auf den Verlauf des Preispfades und meint, daß der Preispfad auf eınem niedrigeren/hoheren Niveau beginnt. 
führt dies bezüglich des Abbauzeitraumes und des Reservenpresses zu Resultaten, die gegensatzlich sınd zu denen, die für eıne Vergroßerung des Steigungsmaßes erhalten werden. Eıne Vergroßerung der Nachfrage (fur $\mathrm{dn}(\mathrm{t})=0$ ) bewirkt über steigende Rohstoffpreıse auch eine Steıgerung des Gegenwartswertes des Reservenpreises. Bei konstanter Diskontrate wachsen die Rohstoffpreise nun schneller, die Fördermengen nehmen schneller $a b$, der Erschöpfungszeitpunkt ist schneller erreicht. Erhöht sich dagegen das Steıgungsmaß1) der Nachfrage bei konstantem choke-off Preis, d.h. der Nachfrageverlauf wird unelastischer, so steigt der Abbauzeitraum, bzw. sinkt der Reservenpreis nicht nur aufgrund des Effektes einer geringeren Nachfrage (ähnlich $d p(T)<0$ ), sondern auch aufgrund der geringeren Elastizität, die zu geringeren Veränderungen der Fördermengen führt.

Schaubild 2.6 stellt die Auswirkungen der parametrischen Änderungen von $\delta, R_{0}$ und c gegenüber.

Die Allokationsprozesse unterscheiden sich in diesem Modell mit Reservenund Rohstoffmarkt nicht von den Ergebnissen der Modelle, die einen Reservenmarkt nicht betrachten.2) Bedeutung kommt diesem zweistufigen Marktkonzept dann zu, wenn vertragliche Vereinbarungen zwischen Reservenbesitzern und Abbauunternehmern modelliert werden sollen, bspw. eine Abhängigkeit der Preise für Abbaurechte von den Rohstoffpreisen vorliegt.

1) $d b>0$ für die Nachfrage $p(t)=p(T)-b q(t)$.

2) Wahlt man in diesem Konzept für Rohstoff- und Reservenmarkt verschiedene Marktformen, so wird das Allokatıonsergebnis bestimmt von dem Verhalten der Marktseite mit der größeren "Marktmacht". So führen bspw. die Mischformen monopsonistischer Reservenmarkt monopolistischer Rohstoffmarkt, monopolistischer Reservenmarkt polypolistischer Rohstoffmarkt und bilaterales Monopol zwischen Reservenbesitzer und Abbauunternehmer zu einem Allokationsergebnis wie es für einen monopolistischen Abbauunternehmer im einstufigen Konzept erhalten werden wurde:

$d q / d t=\delta r(t)\left[2 N^{\prime}(q(t))\right]^{-1}<0, d p / d t=\delta r(t) / 2$. 
Schaubild 2.6: Die intertemporale Ressourcenallokation beiverschiedenen Werten exogener Großen
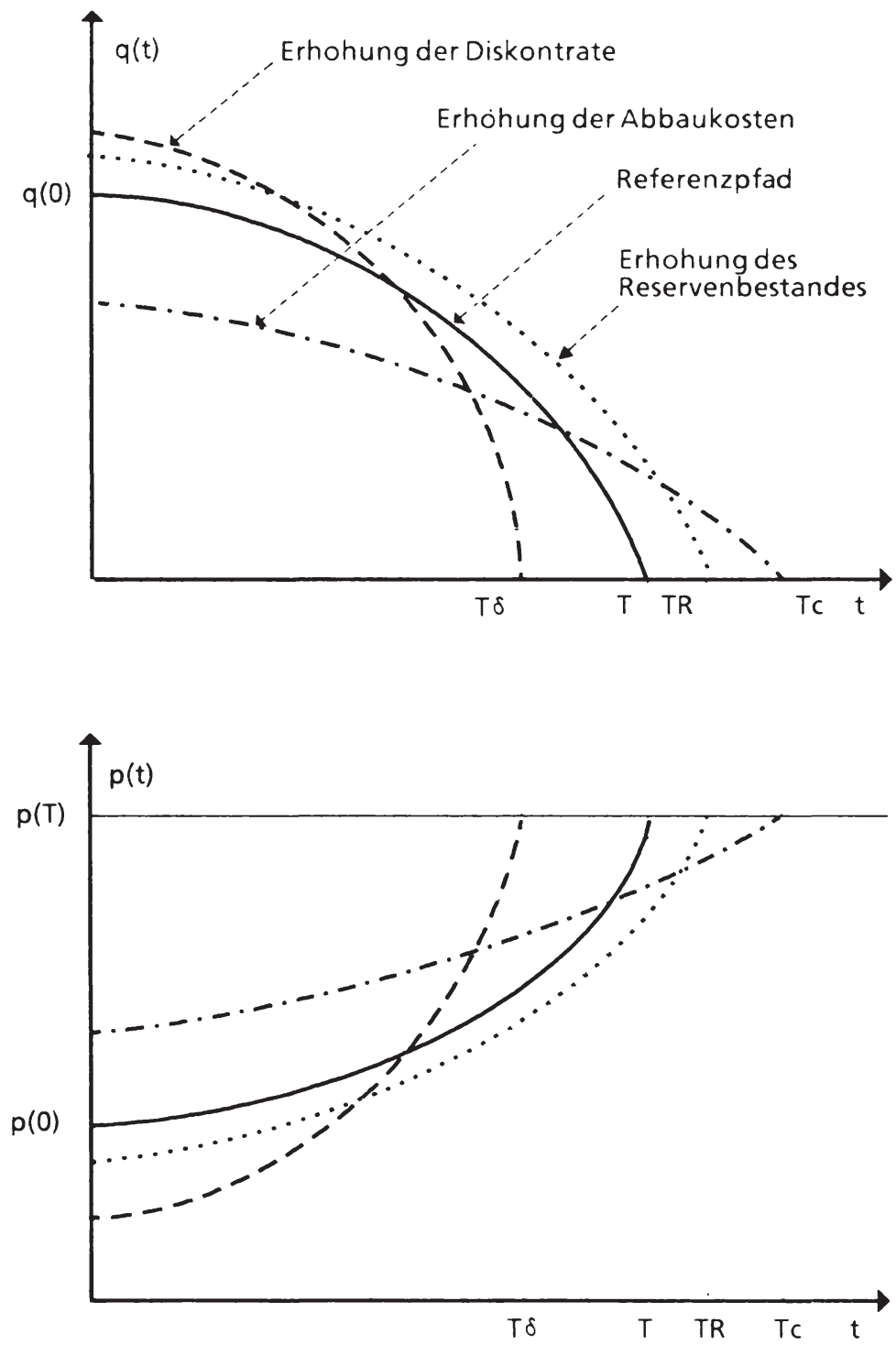
Führt man eine solche Analyse in deterministischen Ressourcenmodellen durch, so kommt man zu dem Resultat, daß die intertemporale Ressourcenallokation von der Gestaltung der Vereinbarungen zwischen Abbauunternehmern und Reservenbesitzern unabhängıg ist. 1) Dıeses Ergebnıs war zu vermuten, wenn man bedenkt, aus welchen Grunden vertragliche Vereinbarungen geschlossen werden. In einem Marktmodell unter Sicherheit bestehen weder unterschiedliche Erwartungen, die ausgenutzt werden könnten, noch existiert eın Rısiko, das es zu begrenzen oder auszuschließen gelte. Führt man Risiko über den Rohstoffmarkt in dieses Marktkonzept ein, so erhält man je nach Gestaltung der Vereinbarungen unterschiedliche intertemporale Allokationsergebnisse. 2)

1) Vgl. Anhang II, wo der Preis für Reserven als vom Rohstoffpreis abhängıge Variable unterstellt wird.

2) Vgl. Abschnitt 6.2. 


\section{Die intertemporale Allokation bei fehlenden Zukunfts- märkten}

Das Fehlen von Zukunftsmärkten führt dazu, daß der Ertrag des Haltens eines Ressourcenbestandes mit Risiko behaftet ist.1) Die Planung des Abbauprogrammes erfolgt aufgrund von Erwartungen über die Preise auf den zukünftigen Kassamärkten. Jeder Abbauunternehmer zeigt unter Risiko ein bestimmtes Risikoverhalten und berücksichtigt dies in seinem Kalkül.

Wie Long 2) ausführt, existiert eıne "verwirrende Vielzahl" von Ansätzen in der Modellbildung zum Verhalten von Wirtschaftssubjekten bei fehlenden Zukunftsmärkten. In dieser Arbeit wird durchgängig der Erwartungsnutzenansatz den Optimierungskalkülen der Wirtschaftssubjekte zugrundegelegt. Lediglich zur graphischen Darstellung wird bei den Zweiperiodenmodellen des fünften Kapitels auf den Mittelwert-Varianz-Ansatz Bezug genommen.

Da nun die Kassamärkte auf der Grundlage von Erwartungen geräumt werden, haben Erwartungsbildungsprozesse einen Einfluß auf die Allokation. Die Diskussion der Preisentwicklungen unter expliziter Betrachtung der Erwartungsbıldung führt zu Stabilitäts- und Ungleıchgewichtsproblemen, wie sie von Heal $(1975,1981)$ und als Kritik auf Heal (1975) von Pflug/Winckler $(1980,1982)$ diskutiert wurden.

Eıne Stabilitätsbetrachtung soll in dieser Arbeit nicht erfolgen. Vielmehr wird den Fragen nachgegangen: Wie reagieren Ressourcenbesitzer und/

\footnotetext{
1) Unsıcherheit wird hier in kalkulierbarer Form als Rısıko modelliert. In Risıkosituatıonen hat man Vorstellungen úber die Häufigkeit des Eintretens moglicher Ereignisse. In Unsicherheitssituationen fehlen diese Vorstellungen.

2) Long(1983), S.71f.
} 
oder Abbauunternehmer auf Risiko? Welche intertemporale Allokation stellt sich aufgrund eines bestimmten Risikoverhaltens ein? Welche Institutionen wirken korrigierend auf einen durch Risiko verursachten Allokationsbias? 1) Zu diesem Zweck werden den Ressourcenbesitzern und/oder Abbauunternehmern rationale Erwartungen über die Preıse der zukünftigen Kassamärkte unterstellt.2) Die subjektiven Verteilungsfunktionen der Abbauunternehmer über die zukünftıgen Preise stimmen im Falle ratıonaler Erwartungen überein mit der objektiven Verteilungsfunktion, d.h. der Erwartungswert des Gleichgewichtspreises für einen bestimmten Zeitpunkt ist für alle Abbaunternehmer gleich. Der Kassamarkt eines Zeitpunktes $t$ ist dann geräumt, wenn das gesamte für diesen Zeitpunkt $t$ aufgrund der Erwartungen geplante Angebot an Rohstoffen gleich ist der vom erwarteten Preis induzierten Nachfrage.3)

Der Aufbau dieses Kapitels folgt dem des vorangegangenen. Ausgelassen wird hier die Betrachtung des nutzenorientierten Ansatzes, da dieser in Abschnitt 6.2.3. eine spezielle Interpretation erfährt und dort diskutiert wird.

1) Korrigierend bezüglich der Referenzallokation einer risikoneutralen Angebotsseite.

2) Rationale Erwartungen können als Grenzfall der adaptiven Erwartungen für eine unendliche Anpassungsgeschwindigkeit angesehen werden. So argumentieren Dasgupta/Heal (1979), S.247.

3) Rationale Erwartungen in dieser Form als ein Gleichgewichtskonzept unter Risiko führen zu einer einfacheren Ermittlung und Darstellung der intertemporalen Allokation als es bei anderen Annahmen über Erwartungsbildungsprozesse der Fall wäre. Grossman (1975), S.255: "...the rational expectations equilibrium is a natural extension of the usual notion of a competitive equilibrium." Siehe hierzu auch Grossman/Stiglitz (1976). 


\subsection{Das Polypol}

Weinsteın/Zeckhauser (1975) analysierten erstmals in eınem Zweiperiodenmodell die Auswirkungen eines fehlenden Zukunftsmarktes auf das Angebotsverhalten eines Ressourcenbesitzers 1 ) und die daraus resultierende Ressourcenallokation.

Dasgupta/Heal (1974) betrachteten Nachfrageunsicherheit derart, daß sie eine Änderung der Rohstoffnachfrage aufgrund des Aufkommens eines Ressourcensubstitutes zu einem unbestimmten Zeitpunkt2) modellierten.

Die Analyse Weinstein/Zeckhausers erweiterte Lewis (1977) auf ein Mehrperiodenmodell und Pindyck (1980, 1981a, 1981b) führte in die Analyse stochastische Prozesse zur Modellierung einer risikobehafteten Nachfrage ein. Der qualitative Unterschied zwischen Lewis und Pindyck besteht in der Betrachtung des Planungskalküls des abbauenden Unternehmers als einstufigen Entscheidungsprozeß bei Lewis bzw. als mehrstufigen Entscheidungsprozeß bei Pindyck. Sowohl Lewis als auch Pindyck verwenden zur Modellierung von Risikoverhalten eine Periodennutzenfunktion. Periodennutzenfunktionen sind jedoch - wie Sinn (1980b) ausführt - zur Modellierung eines risıkoaversen Verhaltens nicht geeignet. Denn aus der Konkavität einer Periodennutzenfunktion für den Fall unter Sicherheit resultiert die Risikoaversion für die Betrachtung unter Risiko als "Nebenprodukt". Zeitpräferenz und Risikopräferenz können nicht voneinander getrennt werden. Als Lösung empfiehlt Sinn3), dem Zielfunktional eine "spezifische Risikopräferenzfunktion" voranzustellen. Über diese kann dann die Risikopräferenz eines Abbauunternehmens variiert werden, ohne daß sich damit auch die Zeitpräferenz ändert.

1) Siehe Abschnitt 5.1. zu einem solchen Ansatz.

2) Diese Art der Unsicherheit über die zukünftige Nach frage wird auch in dem Beitrag Dasgupta/Eastwood/Heal (1978) für die Diskussion der Fördertätigkeit eines ressourcenexportierenden Landes zugrundegelegt.

3) Vgl. $\operatorname{Sinn}(1980 \mathrm{~b}), \mathrm{S} .244$ und $246 \mathrm{f}$. 
Unter der Annahme eines "gewınnmaximierenden" Verhaltens führt das Argument von Sinn dazu, nicht die gesamten diskontierten Werte der Erwartungsnutzen uber den Periodengewinnen als Zielfunktional zu benutzen, sondern den Erwartungsnutzen über den gesamten diskontıerten Periodengewinnen. Alle Modellansatze dieser Arbeit benutzen unter Rısıko elne solche Art der Modellierung von Risikoverhalten.

Was schon in Abschnitt 2 über die Ressourcenallokation der Marktform des Polypols als Referenzallokation ausgesagt wurde, gilt auch unter Risiko. Weinstein/Zeckhauser (1975) und Sweeney (1977) zeigen, daß unter vollständiger Konkurrenz eine risikoneutrale Angebotsseite zu einer sozial optimalen Ressourcenallokation in Termini der erwarteten Preise der zukünftigen Kassamärkte führt. Gegenüber dieser Referenzallokation führt ein risikoaverses Verhalten der Förderunternehmen, welches durch fehlende Zukunftsmärkte induziert wird, zu einem intertemporalen Allokationsbias. Brown/Field und Dasgupta/Heal folgern aus der Abwesenheit von Zukunftsmärkten einen Allokationsbias zugunsten der Zukunft. 1) Zu diesem Ergebnis gelangt auch Lewis (1977), und dieses Ergebnis wird auch in den folgenden Abschnitten bestatigt.

Die Aussage über einen Allokationsbias zugunsten der Zukunft ist abhängig von der Art des zugrundeliegenden Entscheidungsprozesses, der einoder mehrstufig sein kann. Im Falle eines mehrstufigen Entscheidungsprozesses ist die Aussage weiter abhängig davon, ob der unterstellte stochastısche Prozeß linear oder nichtlinear ist. Pindyck (1981c) erhält für einen linearen stochastischen Prozeß bei Risikoaversion einen Allokationsbias zugunsten der Gegenwart.

Der Ansatz Pindycks bestätigt damit die Aussagen des Zweiperiodenmodells von Weinstein/Zeckhauser (1975). Qualitativ gleich sind Pindycks und Weinstein/Zeckhausers Ansätze insofern, als bei beiden im Entscheıdungs-

1) Brown/Field (1979), S.237 und Dasgupta/Heal (1979), S.163. 
zeitpunkt ein Risiko nur über die zukünftıge Nachfrage besteht. Qualitativ unterschiedlıch sınd beide Ansatze hinsıchtlich der Modellierung von Risikoaversıon. Pındyck verwendet eıne Periodennutzenfunktıon, 1) Weinstein/Zeckhauser verwenden eıne Nutzenfunktion wie sie auch in den Ansatzen dieser Arbeit zugrunde liegt.

Es scheınt nicht angebracht zu seın, in Mehrperiodenmodellen unter Risıko die Annahme eines einstufigen Entscheidungsprozesses zu treffen. Eine stochastische Dynamik verlangt closed-loop Strategien als angemessene Problemlösung. Der Unterschied wurde schon kurz angesprochen und besteht darin, 2) daß der Abbauunternehmer mittels der closed-loop Strategie eventuell zusazliche Informatıonen "on-line" verarbeitet und im Falle der open-loop Strategie aufgrund seiner Erwartungen vor Beginn seıner Abbautätigkeit sein Förderprofil festlegt.

Hauptgegenstand der Analyse sind die Allokationswirkungen von Vertragsvereinbarungen über Rohstoffe und über Reserven. Mit Verträgen will man Risiko mindern, will man durch eine Selbstbeschränkung mögliche Verluste vermeiden. Da die modellierten Vertragsvereinbarungen in ihrem Gültigkeitszeitraum als nicht veranderbar unterstellt werden und zu Beginn der Abbautätigkeit abgeschlossen werden, haben spätere Informationen keine Effekte auf die Allokationswirkungen von Verträgen. Als Umsetzung der Gleichgewichtshypothese von Nash kann hier die Hypothese gesetzt werden, daß jedes Wirtschaftssubjekt annimmt, eine Änderung selner Informationsmenge habe keinen Einfluß auf die Preise. 3) Auf das aufwendigere Lösungskonzept der closed-loop Strategie kann deshalb zugunsten der open-loop Strategie verzichtet werden.

1) Die Annahme einer Periodennutzenfunktion liefert eine separable Zielfunktion, die Voraussetzung für die stochastische dynamische Programmierung ist. Da in den Ansätzen dieser Arbeıt die Separabılität der Nutzenfunktion in der Zeit nicht unterstellt ist, kann auch aus formalen Grunden eine closed-loop Losung mittels der stochastischen dynamischen Programmierung nicht ermittelt werden.

2) In Zweiperiodenmodellen sind open- und closed-loop Lösungen identisch.

3) Vgl. Grossman/Stiglitz (1976), S.247. 


\subsubsection{Der gewinnorientierte Ansatz}

Es gelten die Annahmen, die teilweise aus Abschnitt 2 bekannt sind, aber noch einmal genannt werden sollen.

Ein Abbauunternehmer besitze den bekannten Ressourcenbestand $R_{0}$. Diesen baue er mit den Kosten $C(q(t))\left[C^{\prime}(q(t)), C^{\prime \prime}(q(t))>0, C^{\prime \prime \prime}(q(t))=0\right]$ ab. $E_{1}-$ ne Lagerhaltung der abgeba: Iten Ressourcenmengen $q(t)$ finde nicht statt.

Die Abbaumengen der Periode $t$ werden auf dem Kassamarkt in $t$ unter vollkommener Konkurrenz zum Preis $p(t)$ verkauft. 1) Da Zukunftsmärkte nicht existieren, plant der Abbauunternehmer seine Abbauentscheidungen aufgrund von Erwartungen uber die Preise auf den zukünftigen Kassamärkten. $p(t)$ ist im Kalkül des Abbauunternehmers eine Zufallsvariable.

Der Abbauunternehmer habe eine Nutzenfunktion U (Risikopräferenzfunktion), die über seinem Gewinn $G$ zweimal stetig differenzierbar sei mit $\mathrm{dU} / \mathrm{dG}=\mathrm{U}^{\prime}>0$ und $\mathrm{d}^{2} \mathrm{U} / \mathrm{dG}^{2}=\mathrm{U}^{\prime \prime}<0$ bei Risikoaversion, $U^{\prime \prime}=0$ bei Risikoneutralität, $U^{\prime \prime}>0$ bei Risikofreude.2) $G$ ist die Summe aller Gegenwartswerte der Periodengewinne aus den Ressourcenverkäufen während des Abbauzeitraumes.

Die Diskontrate $\delta$ sei der Zinssatz eines vollkommenen Kapitalmarktes, auf dem die Erträge aus der Abbautätigkeit sicher angelegt werden können.

Als Zielfunktion wird der Erwartungsnutzen des Gewinnes verwendet.

Das Optimierungsproblem des Abbauunternehmers lautet dann mit

$$
G={ }_{0} \int^{T}[p(t) q(t)-C(q(t))] e^{-\delta t d t}
$$

1) Fett gedruckte Variable stehen für Zufallsvarıable.

2) Für die Darstellung mit der Prattschen Rısikoprämie wird U'" $=0$ unterstellt. 


$$
\begin{aligned}
& \max _{(q(t), T)} E[U(G)] \\
& \text { u.d.B. } \quad R_{0} \geq{ }_{0} \int^{\top} q(t) d t, R_{0} \text { gegeben, } q(t) \geq 0 .
\end{aligned}
$$

Aus der Lagrangefunktion

$$
L=E[U(G)]+\lambda\left[R_{0}-{ }_{0} \int^{\top} q(t) d t\right]
$$

ergeben sich die folgenden notwendigen Bedingungen

$$
\begin{aligned}
& E\left\{U^{\prime}\left[p(t)-C^{\prime}(q(t))\right]\right\} e^{-\delta t-\lambda} \quad\left\{\begin{array}{cc}
=0 & q(t)>0 \\
\text { für } & \\
\leq 0 & q(t)=0
\end{array}\right. \\
& \left\{\begin{array}{lll}
=0 & \lambda>0 \\
& \text { für } & \\
\geq 0 & \lambda=0
\end{array}\right.
\end{aligned}
$$

(3.3)(iii) $\quad E\left\{U^{\prime}[p(T) q(T)-C(q(T))]\right\} e^{-\delta T}-\lambda q(T)=0$.

Für positive Abbaumengen folgt aus 3.3 (i)

$$
E\left\{U^{\prime}\left[p(t)-C^{\prime}(q(t))\right]\right\}=\lambda e^{\delta t}
$$

Der Erwartungsnutzen des Perıodengrenzgewinnes wächst mit der Diskontrate $\delta$. Dies ist die Hotelling-Regel bei Risiko. ${ }^{1)}$

Die Transversalitätsbedingung 3.3 (iii) liefert mit 3.4 für $q(T)>0$

1) Zu diesem Resultat siehe auch Lewis (19;7), S.114. 


$$
E\left\{U^{\prime}\left[p(T)-C(q(T)) q(T)^{-1}\right]\right\}=E\left\{U^{\prime}\left[p(T)-C^{\prime}(q(T))\right]\right\} .
$$

Der Ressourcenabbau wird in T eingestellt, wenn der Erwartungsnutzen des Durchschnittsgewinnes gleich dem Erwartungsnutzen des Grenzgewınnes in T ist. Das ist der Fall, wenn die Durchschnitts- gleich den Grenzkosten des Abbaus sind.1)

Die Ressource wird in T erschöpft, wenn nach 3.3 (ii) $\lambda>0$ gilt.

Zur Betrachtung unterschiedlichen Risikoverhaltens wird Gleichung 3.4 mit der Beziehung

$$
\operatorname{cov}\left[U^{\prime}, p(t)\right]=E\left[U^{\prime} p(t)\right]-E\left(U^{\prime}\right) E[p(t)]
$$

umgeschrieben zu

$$
E[p(t)]=\lambda e^{\delta t E\left(U^{\prime}\right)-1}+C^{\prime}(q(t))-\operatorname{cov}\left[U^{\prime}, p(t)\right] E\left(U^{\prime}\right)^{-1} .
$$

Hier hat man das stochastische Analogon zum deterministischen Fall, wo der Marktpreis der Ressource gleich der Summe aus Opportunitäts- und Grenzabbaukosten ist.

Risikoaverse und risikofreudige Abbauunternehmer berücksichtigen bei ihrer Preisbildung neben den Opportunitäts- und Grenzabbaukosten auch "Kosten" des Risikoverhaltens.

1) Gleichung 3.5 ist äquivalent zu

$$
\begin{gathered}
E\left(U^{\prime}\right)\left\{E[p(T)]+\operatorname{cov}\left(U^{\prime}, p(T)\right) E\left(U^{\prime}\right)-1-C(q(T)) q(T)^{-1}\right\} \\
=E\left(U^{\prime}\right)\left\{E[p(T)]+\operatorname{cov}\left(U^{\prime}, p(T)\right) E\left(U^{\prime}\right)^{-1}-C^{\prime}(q(T))\right\}
\end{gathered}
$$

und damit $C(q(T)) q(T)-1=C^{\prime}(q(T))$. 
Fur unterschiedliches Risikoverhalten gilt hinsichtlich der Kovarianz 1)

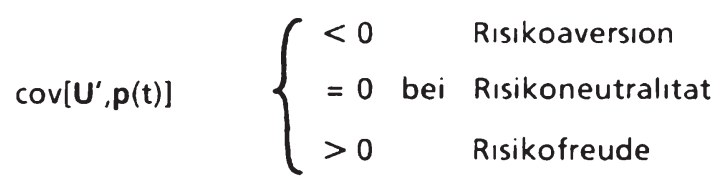

Der risikoaverse (risikofreudige) Abbauunternehmer wird nach 3.6 zu einem Angebot auf dem zukunftigen Kassamarkt in t bereit sein, wenn er einen Preis erwarten darf, der seine Opportunitätskosten um den Betrag $\operatorname{cov}\left[U^{\prime}, p(t)\right] E\left(U^{\prime}\right)-1$ übersteigt (unterschreitet).2)

Ein risikoaverser Abbauunternehmer wird eine kleinere Abbaumenge wählen als jene, bei der der erwartete Preis gleich den Opportunitäts- plus Grenzabbaukosten ist. Der risikoaverse Unternehmer "schützt" sich damit vor einer mit Risiko behafteten "Überproduktion" .3)

Für einen risikofreudigen Abbauunternehmer gilt das Gegenteil. Er wählt eine großere Abbaumenge als jene, bei der die Gleichheit zwischen erwartetem Preis und Opportunitäts- plus Grenzabbaukosten gilt.

Greift man auf die von Pratt 4 ) eingeführte Definiton einer Risikoprämie zurück, dann ist der Kovarianzterm einer weiteren Interpretation zugänglich.

Die Risikopramie $Z$ ist in diesem Modellrahmen so zu bestimmen, daß gilt

1) Um dies abzuleiten, ist der Effekt einer Änderung von $p(t)$ auf $U^{\prime} z U$ betrachten:

$d U^{\prime}(G) / d p=U^{\prime \prime}(d G / d p)$ mit $d G / d p>0$ und $U^{\prime \prime}<0$ bei Risikoaversion, $U^{\prime \prime}=0$ bei Risikoneutralitat, $U^{\prime \prime}>0$ bei Rısıkofreude.

2) $\operatorname{cov}\left(U^{\prime}, p(T)\right) E\left(U^{\prime}\right)-1$ wird in Abschnitt 6.1.1. als "subjektiver Risıkopreis" interpretiert.

3) Zum statischen Fall vgl. bspw. Sandmo (1971).

4) Pratt (1964), S.124. 


$$
U[E(G-Z)]=E[U(G)]
$$

$Z$ ist der Betrag, um den der erwartete Gewinn vermindert werden kann, so daß der Abbauunternehmer indifferent wird zwischen der sicheren Gewinneinnahme $E(G)-Z$ und der unsicheren Gewinnaussicht $G$.

Differenziert man 3.7 nach $q(t)$, so wird erhalten

$$
U^{\prime}\{E[(d G / d q)-(d Z / d q)]\}=E\left[U^{\prime}(d G / d q)\right] .
$$

Unter Verwendung der notwendigen Bedingung 3.3 (i) und deren Umformung 3.6 ergibt sich

$$
d Z / d q=\lambda e^{\delta t}\left[U^{\prime}-E\left(U^{\prime}\right)\right]\left[U^{\prime} E\left(U^{\prime}\right)\right]^{-1}-\operatorname{cov}\left[U^{\prime}, p(t)\right] E\left(U^{\prime}\right)^{-1} .
$$

Mit der Annahme $U^{\prime \prime \prime}=0$ folgt $U^{\prime}=E\left(U^{\prime}\right)$, und weiter

$$
d Z / d q=-\operatorname{cov}\left[U^{\prime}, p(t)\right] E\left(U^{\prime}\right)^{-1} .
$$

Das Vorzeichen ermittelt sich zu

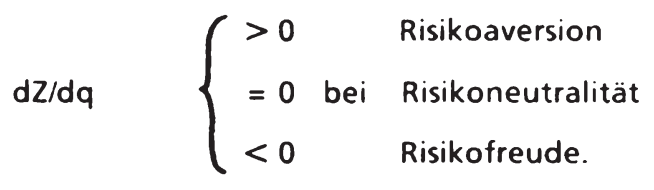

Gleichung 3.6 kann damit umgeschrieben werden zu

$$
E[p(t)]=\lambda e^{\delta t E\left(U^{\prime}\right)-1}+C^{\prime}(q(t))+d z / d q,
$$

und ist wie folgt zu interpretieren:

Hat der risikoaverse Abbauunternehmer sein Optimum realisiert, dann ist er indifferent zwischen

(1) der Verhaltensweise, wie sie in der Interpretation zu Gleichung 3.6 erfolgt. Dort wird zu einem mit Risiko behafteten Preis $E[p(t)]$ eine Abbaumenge angeboten, die geringer ist als die Menge nach der "Press-gleich-Opportunitäts-plus-Grenzabbaukosten-Regel". 
(2) der Verhaltensweise, bei derselben Abbaumenge wie unter (1) Risiko zu vermeiden, indem ein sicherer aber geringerer Preis als der erwartete Preis $E[p(t)]$ akzeptiert wird.

Ein risikofreudiger Abbauunternehmer wird im Falle einer sicheren Preiszusage einen Preis über dem erwarteten Preis $E[p(t)]$ fordern.

Der risikovermeidende Preis hat den Wert $E[p(t)]-d Z / d q$ und wäre zu interpretieren als der Preis auf einem Terminmarkt mit dem Fälligkeitsdatum $t$ oder als der Preis in einem Liefervertrag für die Periode $t$.

Zur Ermittlung der Abbaupfade und der Preisentwicklungen bei unterschiedlichem Risikoverhalten wird die Nachfrage in die Betrachtung mit einbezogen.

Für die zeitinvariante Nachfrage gelte 1)

$$
p(t)=N(q(t)) u(t), \text { mit } N^{\prime}(q(t))<0 \text { und } N^{\prime \prime}(q(t))=0 \text {. }
$$

Hierin sei $u(t)$ eine nichtnegative, identisch und unabhängig über die Zeit verteilte Zufallsvariable mit $E[u(t)]=1$ und $\operatorname{var}[u(t)]=\sigma^{2}$.

Die Ressourcennachfrager halten keine Läger.

Bisher wurde ein einzelner Ressourcenanbieter betrachtet. Dieser sei repräsentativ für die Gesamtheit der Anbieter, die eine konstante AnzahI von Lagerstätten abbauen. Alle Ressourcenanbieter besitzen rationale Erwartungen über die zukünftigen Kassamarktpreise und zeigen ein einheitliches Risikoverhalten.

Betrachtet man Gleichung 3.6 als die Angebotsfunktion des Marktes, 2 ) dann kann zusammen mit der Nachfrage 3.10 die zeitliche Entwicklung der gleichgewichtigen Abbaumengen abgeleitet werden.

1) Vgl. hierzu Lewis (1977), S.113.

2) Es wird nicht explizit zwischen der aggregierten Angebotsmenge für den Markt und den dafür ursächlichen infinitesimal kleinen Abbaumengen unterschieden. 
An dieser Stelle empfiehlt sich die Definition einer Variablen, die in Abhàngigkeit vom Risikoverhalten den Vergleich der Allokationsergebnısse verschiedener Modellvarianten erleichtert. 1)

Definiert man

$$
\Gamma=E[p(t)]\left\{E[p(t)]+\operatorname{cov}\left[U^{\prime}, p(t)\right] E\left(U^{\prime}\right)^{-1}\right\}^{-1},
$$

dann folgt je nach Risikoverhalten

$$
\Gamma \quad \begin{cases}>1 & \text { Risikoaversion } \\ =1 \text { bei } & \text { Risikoneutralität } \\ <1 & \text { Risikofreude }\end{cases}
$$

$\Gamma$ ist $z u$ interpretieren als ein durch Risiko induzierter Bias im Angebotsverhalten eines Abbauunternehmers.

Die zu 3.6 äquivalente Schreibweise lautet damit

$$
E[p(t)] \Gamma^{-1}-C^{\prime}(q(t))=\lambda e^{\delta t E\left(U^{\prime}\right)-1}
$$

und führt zu2)

$$
\begin{aligned}
& \mathrm{dq} / \mathrm{dt}=\lambda \mathrm{e}^{\delta t E}\left(\mathrm{U}^{\prime}\right)^{-1}\left\{\mathrm{~N}^{\prime}(\mathrm{q}(\mathrm{t})) \Gamma^{-1}-\mathrm{C}^{\prime \prime}(\mathrm{q}(\mathrm{t}))+\lambda \mathrm{e}^{\delta t E}\left(\mathrm{U}^{\prime}\right)-2\left(\mathrm{dE}\left(\mathrm{U}^{\prime}\right) / \mathrm{dq}\right)\right\}-1 \\
& \mathrm{dq} / \mathrm{dt}<0 .
\end{aligned}
$$

1) Vgl. Sutinen (1980), S.172. Dort wird ein $\Delta=E\left(U^{\prime}\right) E\left[U^{\prime} u(t)\right]^{-1}$ ohne eine entsprechende ökonomische Interpretation eingeführt. Die hier definierte Relation ist einer Interpretatıon zuganglıch und formal äquivalent der von Sutinen.

2) Vgl. Anhang III. Unterstellt wurde $d \Gamma / d t=0$, dies ist äquivalent zu der Annahmedcov( $\left.U^{\prime}, p(t)\right) / d t=0$. 
Für die Entwicklung der Preiserwartungen ergıbt sich

$$
d E[p(t)] / d t=d p / d t=N^{\prime}(q(t))(d q / d t)>0 .
$$

Hieraus folgt unter Beachtung der Vorzeichen und Größenrelatıonen

$$
\begin{aligned}
& (d q / d t)_{F}<(d q / d t)_{N}<(d q / d t)_{A}<0 \text { und } \\
& (d p / d t)_{F}>(d p / d t)_{N}>(d p / d t)_{A}>0 .
\end{aligned}
$$

Eine risikoaverse (A) Angebotsseite führt zu kleineren Veränderungen der Abbaumengen als eine risikoneutrale $(N)$ Angebotsseite und diese wiederum zu kleineren Veränderungen als eine risikofreudige (F) Angebotsseite.

Bei gegebenem Ressourcenbestand liegen damit auch die Niveaurelationen der Abbaupfade fest.

Fordert man neben den Stromgleichgewichten zwischen Angebot und Nachfrage für jede Periode auch die Bedingung, daß das Ressourcenlager zum gleichen Zeitpunkt erschöpft sein soll, zu dem die Nachfrage auf Null sinkt, dann muß bei kleineren zeitlichen Veränderungen der Abbaumengen auch die Abbaumenge zu Anfang kleiner sein.

Aus

$$
\begin{aligned}
& q(0)_{A}<q(0)_{N}<q(0)_{F} \text { folgt mit } \\
& (d q / d t)_{F}<(d q / d t)_{N}<(d q / d t)_{A}<0
\end{aligned}
$$

für die Erschöpfungszeitpunkte $T_{A}>T_{N}>T_{F}$.

Es kann die Aussage gemacht werden, daß eine risikoaverse Angebotsseite einen gegebenen Ressourcenbestand später erschöpft als eine risikoneutrale und diese wiederum später als eine risikofreudige Angebotsseite.

Benutzt man den Abbaupfad risikoneutraler Ressourcenbesitzer als Referenzpfad, dann zeigt der Abbaupfad risikoaverser Ressourcenbesitzer einen Allokationsbias zugunsten der Zukunft und der Abbaupfad der risikofreudigen Ressourcenbesitzer eınen Allokationsbias zugunsten der Gegenwart. 
Die Abbaupfade 1) mit den zugehörigen Pfaden der erwarteten Preisentwicklung zeıgt Schaubild 3.1.

Es existieren Zeıtpunkte $t^{\prime}, t^{\prime \prime}$ und $t^{\prime \prime \prime}$ für die die Mengenrelationen 2) gelten $q(t)_{A}\left\{\begin{array}{l}\leq \\ q(t)_{N}\end{array} \quad\right.$ für $\left\{\begin{array}{l}0 \leq t \leq t^{\prime} \\ t^{\prime}<t \leq T_{N}<T_{A}\end{array}\right.$ $q(t)_{N}\left\{\begin{array}{l}\leq \\ q(t)_{F}\end{array} \quad\right.$ für $\left\{\begin{array}{l}0 \leq t \leq t^{\prime \prime} \\ t^{\prime \prime}<t \leq T_{F}<T_{N}\end{array}\right.$ $q(t)_{A}\left\{\begin{array}{l}\leq \\ q(t)_{F}\end{array} \quad\right.$ für $\left\{\begin{array}{l}0 \leq t \leq t^{\prime \prime \prime} \\ t^{\prime \prime \prime}<t \leq T_{F} .\end{array}\right.$

1) Für eine risikoneutrale Angebotsseite gilt $\mathrm{d}^{2} \mathrm{q} / \mathrm{dt} 2<0$. Bei anderem $\mathrm{Ri}$ sikoverhalten folgt dies nicht eindeutig. Im Schaubild wurde jedoch auch für Risikoaversion und Risikofreude $\mathrm{d}^{2} \mathrm{q} / \mathrm{dt}^{2}<0$ unterstellt.

2) Vgl. die Darstellung bei Lewis (1977), S.114 für ein diskretes Modell mit einer Periodennutzenfunktion. 
Schaubild 3.1: Die intertemporale Ressourcenallokation für unterschiedlıches Risıkoverhalten

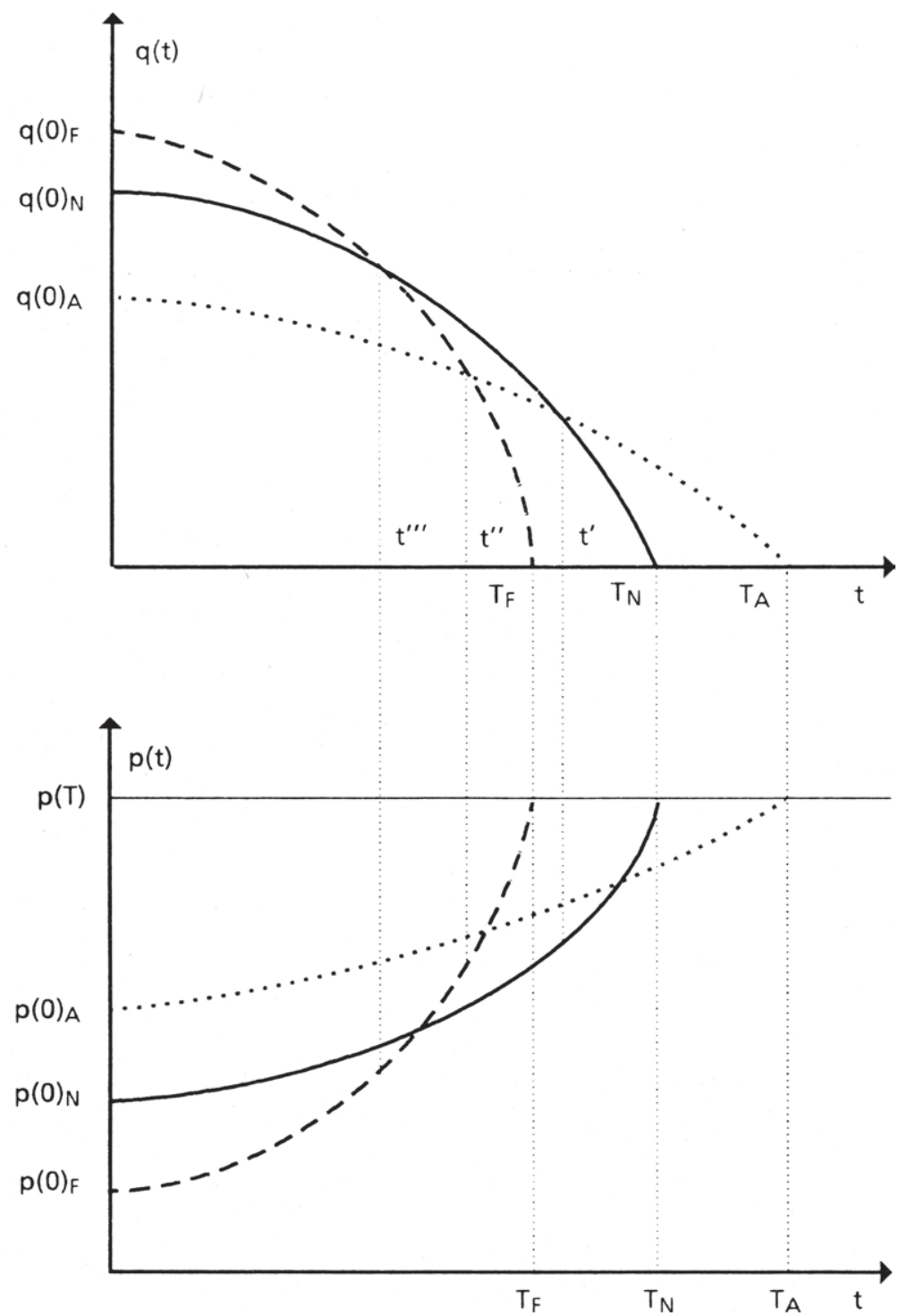


Die Lage der Zeitpunkte $t^{\prime}, t^{\prime \prime}, t^{\prime \prime \prime}$ im q-t Diagramm impliziert bestimmte Relationen der Preispfade in diesen Zeitpunkten. 1)

Man hat in

$$
\begin{aligned}
& t^{\prime}: q\left(t^{\prime}\right)_{A}=q\left(t^{\prime}\right)_{N} \text { und } p\left(t^{\prime}\right)_{A}>p\left(t^{\prime}\right)_{N} \\
& t^{\prime \prime}: q\left(t^{\prime \prime}\right)_{N}=q\left(t^{\prime \prime}\right)_{F} \text { und } p\left(t^{\prime \prime}\right)_{N}>p\left(t^{\prime \prime}\right)_{F} \\
& t^{\prime \prime \prime}: q\left(t^{\prime \prime \prime}\right)_{A}=q\left(t^{\prime \prime \prime}\right)_{F} \text { und } p\left(t^{\prime \prime \prime}\right)_{A}>p\left(t^{\prime \prime \prime}\right)_{F} .
\end{aligned}
$$

Bei gleicher Ausbringungsmenge fordert der risikoaverse Abbauunternehmer einen höheren Preis als der risikoneutrale Abbauunternehmer und dieser wiederum einen höheren Preis als der risikofreudige Abbauunternehmer.

Die Analyse zeigt, daß das Risikoverhalten bedeutenden Einfluß auf die intertemporale Ressourcenallokation hat.

Verglichen mit einem risikoneutralen Ressourcenbesitzer verschiebt der risikoaverse Ressourcenbesitzer den Abbau in die Zukunft. Er senkt seine Abbaumengen in den Anfangsperioden, wo relativ große Veränderungen der Abbaumengen und der Erträge vorliegen würden und er erhöht seine $A b-$ baumengen in späteren Perioden, wo relativ kleine Veränderungen der $A b$ baumengen vorliegen würden. Auf diese Weise senkt der risikoaverse Abbauunternehmer die Varianz seines Gewinnes. Das Risiko wird intertemporal diversifiziert. Denn es gilt für eine Periode $t$ :

$$
\operatorname{var}[G(t)]=\left[q(t) e^{-\delta t}\right]^{2} \operatorname{var}[p(t)] .
$$

Je risikoaverser die Angebotsseite sich verhält, um so flacher verlaufen der Abbaupfad und der zugehörige Preispfad. Der Abbaupfad beginnt auf einem niedrigeren, der Preispfad auf einem höheren Niveau.

1) Dies folgt aus den notwendigen Bedingungen 3.3 und der Annahme rationaler Erwartungen. Die Preispfade stehen für die zeitliche Entwicklung der erwarteten Marktpreise. 
Lewis (1977) kommt zu dem gleichen Ergebnis. In einem diskreten Modell mit eıner Perıodennutzenfunktion erhält er ebenfalls überkonservierenden Ressourcenabbau fur einen risikoaversen Abbauunternehmer.

Weınsteın/Zeckhauser (1975) In einem Zweiperiodenmodell1) undPindyck (1981) in einem stetıgen Modell erhalten unterkonservierenden Ressourcenabbau.2) Diese Resultate scheinen widersprüchlich, doch klärt sich dies, wenn man betrachtet, wie die Preise der Kassamärkte in den Entscheidungsproze $\beta$ des Abbauunternehmers der jeweiligen Modelle eingehen.

In Lewis Modell und in diesem Abschnitt sind alle Kassamarktpreise Zufallsvariable im Entscheidungskalkul. Auch der Press des Kassamarktes in $\mathrm{t}=0$. Indem der Abbauunternehmer überkonserviert, versucht er, über die Zeit zu diversifizieren. Alle Preise sind mit dem gleichen Risiko behaftet. ${ }^{3)}$ Alle Entscheidungen sind in der ersten Periode zu treffen. ${ }^{4)}$

1) Vgl. auch Abschnitt 5.1.

2) Weinstein/Zeckhauser verwenden eine Nutzenfunktion über dem Gesamtgewinn. In Pindycks Zielfunktion geht eine Periodennutzenfunktion ein. Die Nachfrage wird als stochastischer Prozeß modelliert. Das oben genannte Ergebnis gılt für $U^{\prime \prime}(G)=0$ und für einen stochastischen Prozeß, der in der Störgröße linear ist.

3) Läßt man in dem hier vorgestellten Ansatz ein im Zeitablauf wachsendes Risiko zu, (d $\Gamma / \mathrm{dt}) / \Gamma>0$, dann führt dies bei risikoaverser Angebotsseite zu einem schnelleren Ressourcenabbau als bei $(\mathrm{d} \Gamma / \mathrm{dt}) / \Gamma=0$. Gleichung 3.11 würde für $(\mathrm{d} \Gamma / \mathrm{dt}) / \Gamma><0$ lauten

$$
\begin{aligned}
& d q / d t=\lambda e^{\delta t E\left(U^{\prime}\right)-1\left\{N(q(t))-\Gamma C^{\prime}(q(t))+N(q(t))[(d \Gamma / d t) / \Gamma]\right\} .} \\
& \left\{N^{\prime}(q(t))-\Gamma\left[C^{\prime \prime}(q(t))-\lambda e^{\left.\left.\delta t E\left(U^{\prime}\right)-2\left(d E\left(U^{\prime}\right) / d q\right)\right]\right\}-1<0 .}\right.\right.
\end{aligned}
$$

Eıne eindeutige Aussage ist nun nicht mehr möglich. Der risikoaverse Unternehmer kann nun schneller oder langsamer als der risıkoneutrale Unternehmer abbauen.

4) Open-loopOptımierung. 
In Zweiperiodenmodellen dagegen ist der Press des Kassamarktes in $\mathrm{t}=0$ keine Zufallsvariable. 1) Mit Risiko behaftet ist nur der Preis des Kassamarktes in $\mathrm{t}=1$. Der risıkoaverse Abbauunternehmer wird zugunsten der Gegenwart abbauen. Denn in diesem Modellkontext kann die Varıanz des Gewinnes reduziert werden, Indem der Ressourcenabbau zugunsten der Gegenwart umverteilt wird.

Das gleiche Abbauverhalten zeigt der Ressourcenbesitzer in Pindycks Modellstruktur, wo die Entscheidung immer nur von Periode zu Perıode unter Kenntnis der bıs dahin eingetretenen Ereignisse getroffen wird.2) Hınzu kommt die Tatsache, daß Pindyck das Rısiko im Zeıtablauf zunehmen laßt.

\subsubsection{Der umsatzorientierte Ansatz}

Mit Ausnahme der Zielfunktion gelten weiterhin die Annahmen des vorigen Abschnittes.

Für die Manager wird der Umsatz nun zur Zufallsvariablen. Sie maximieren deshalb den Erwartungsnutzen des gesamten Umsatzes in Gegenwartswerten unter der Nebenbedingung eines gegebenen Ressourcenbestandes und eines geforderten Mindestgewinnes. Dem geforderten Mindestgewinn entspricht ein Mindestnutzenniveau, das die Manager in Erwartungsnutzeneinheiten des realisierten Gewinnes nicht unterschreiten dürfen. Sowohl für die Manager als auch für die Eıgentümer wırd dieselbe Nutzenfunktion unterstellt. Es gelte die Nutzenfunktion, wie sie in Abschnitt 3.1.1. formuliert wurde.

Die Unternehmensführung sieht sich damit vor das folgende Optimierungsproblem gestellt:

1) Vgl. Abschnitt 5.1.

2) Closed-loop Optimierung. Im Zweıperıodenmodell sind die Lösungen von open-und closed-loop Optimierung identisch. 
(3.13) $\max E\left\{U\left[\int_{0} \int^{\top} p(t) q(t) e^{-\delta t d t}\right]\right.$ $(q(t), T)$

u.d.B. $\quad R_{0} \geq{ }_{0}{ }^{T} q(t) d t, R_{0}$ gegeben, $q(t) \geq 0$

$$
U\left(G_{0}\right) \leq E\left\{U\left[\int_{0} \int^{T}[p(t) q(t)-C(q(t))] e^{-\delta t d t}\right]\right\}
$$

An notwendigen Bedingungen erhält man über die Lagrangefunktion 1)

(3.14)(i) $E\left[U^{\prime} p(t)\right] e^{-\delta t}-\lambda+\mu E\left\{U^{\prime}\left[p(t)-C^{\prime}(q(t))\right]\right\} e^{-\delta t} \begin{cases}=0 & q(t)>0 \\ \text { für } & \\ \leq 0 & q(t)=0\end{cases}$

(3.14)(ii) $R_{0}-{ }_{0} \int^{\top} q(t) c i t$

$$
\left\{\begin{array}{lll}
=0 & \lambda>0 \\
& \text { für } & \\
\geq 0 & \lambda=0
\end{array}\right.
$$

(3.14)(III) $\left.E\left\{U I_{0} J^{T}[p(t) q(t)-C(q(t))] e^{-\delta t d t}\right]\right\}-U\left(G_{0}\right)\left\{\begin{array}{ccc}=0 & \mu & >0 \\ & \text { für } & \\ \geq 0 & \mu=0\end{array}\right.$

(3.14)(iv) $E\left\{U^{\prime}[p(T) q(T)]\right\} e^{-\delta T}-\lambda q(T)+\mu E\left\{U^{\prime}[p(T) q(T)-C(q(T))]\right\} e^{-\delta T}=0$.

1) $L=E\left\{U\left[{ }_{0} S^{\top} p(t) q(t) e^{-\delta t d t}\right]\right\}+\lambda\left[R_{0}-{ }_{0}{ }^{\top} q(t) d t\right]+$ $\mu\left\{E\left\{U\left[\int_{0} \int^{\top}[p(t) q(t)-C(q(t))] e^{-\delta t d t}\right]\right\}-U\left(G_{0}\right)\right\}$ 
Planen die Manager bei einer gegebenen Erwartungshaltung und einem bestımmten Risıkoverhalten eın posıtives Angebot auf dem Kassamarkt in $t$, so geschieht dies nach 1)

$$
(1+\mu) E[p(t)] \Gamma^{-1}=\lambda e^{\delta t E\left(U^{\prime}\right)^{-1}+\mu C^{\prime}(q(t)) .}
$$

Ein Vergleich der Ausbringungsmengen beim Abbau zwischen dem gewinnorientierten Unternehmer (G) und dem umsatzorientierten Manager (W) für eine gegebene und gleiche Erwartungshaltung und identisches Risikoverhalten führt zu der eindeutigen Aussage, daß aus

$$
\left[\lambda e^{\delta t E}\left(U^{\prime}\right)^{-1}\right]_{W}<\left[\lambda e^{\delta \delta t E\left(U^{\prime}\right)-1}\right]_{G} \text { folgt } q(t)_{W}>q(t)_{G} .
$$

D.h. der umsatzorientierte Manager wählt bei gleichem Risikoverhalten und gleicher Erwartungshaltung für einen bestimmten Zeitpunkt eine größere Abbaumenge als der gewinnorientierte Unternehmer. Dieses Ergebnis folgt ebenfalls unter Sicherheit. Die für eine gewinnorientierte Angebotsseite getroffenen vergleichenden Aussagen bei unterschiedlichem Risikoverhalten bleiben bestehen.

Für die Nachfrageseite der Kassamärkte gelte die Funktion 3.10. Die einzelnen unternehmensführenden Manager besitzen rationale Erwartungen über die gleichgewichtigen Preise der Rohstoffmärkte.

Die zeitliche Entwicklung der erwarteten, markträumenden Rohstoffpreise und der zugehörigen Rohstoffmengen ermitteln sich zu

1) Für $\lambda, \mu>0$ folgt dies aus 3.14 (i). 
(3.16) $\quad d q / d t=\delta\left[N(q(t)) \Gamma^{-1}-\mu(1+\mu)^{-1} C^{\prime}(q(t))\right]$

$$
\begin{aligned}
\left\{N^{\prime}(q(t)) \Gamma^{-1}-\mu(1+\mu)-1-C^{\prime \prime}(q(t))+\lambda e^{\delta t E}\left(U^{\prime}\right)-2\left[d E\left(U^{\prime}\right) / d q\right](1+\mu)^{-1}\right\}-1 & <0
\end{aligned}
$$

(3.17) $\quad d p / d t=N^{\prime}(q(t))(d q / d t)>0$.

Vergleicht man unter Risiko die Allokationsergebnisse zwischen gewinnund umsatzorientierter Angebotsseite, 1) so gelangt man auch hier zu der Aussage, daß umsatzmaximierende Unternehmen einen gegebenen Ressourcenbestand schneller abbauen als gewinnorientierte Unternehmer, nun allerdings mit einem durch Risıko induzierten Allokationsbias.

1) Siehe hierzu Fußnote 1 auf S. 25. 


\subsection{Das Monopol}

Den einzigen Beitrag 1) zur Analyse der intertemporalen Allokation unter der Marktform des Monopols bei Nachfragerisiko, also bei fehlenden Zukunftsmärkten, liefert Pindyck (1980).2) Neben der Annahme eines stochastischen Prozesses für die Nachfrage unterstellt Pindyck auch einen stochastischen Prozeß für den Reservenbestand. Das Abbauunternehmen verhält sich risikoneutral, maximiert also den Erwartungswert der gesamten diskontierten Periodengewinne. Ohne Berücksichtigung des Reservenbestandsrisıkos erhält Pindyck das Ergebnis, daß die erwartete Veränderung des Grenzerlöses gleich der Veränderung des Grenzertrages unter Nachfragesicherheit ist. Auf das (erwartete) Preisprofil hat das Nachfragerisiko keinen Einfluß. Einfluß hat es jedoch auf das Mengenprofil des Abbaus. BeI einer linearen Nachfrage wird der Reservenbestand schneller erschöpft. Bei nichtlinearer Nachfrage ist keine eindeutige Aussage möglich.

Abweichend zu Pindyck wird hier Risikoverhalten im Erwartungsnutzenkonzept modelliert. Der Reservenbestand ist für den Monopolisten nicht mit Risiko behaftet. Ferner wird die Nachfrage nicht als stetiger stochastischer Prozeß mit im Zeitablauf zunehmender Varianz vorgegeben, sondern in der zeitinvarianten Form von Gleichung 3.10.

Für die Abbaukosten und die Nutzenfunktion des Monopolisten gelten die Annahmen des Abschnitts 3.1.1.

Das Angebotsverhalten des Monopolisten wird ohne und mit Preisdifferenzierung diskutiert. Gezeigt werden kann, daß Preisdifferenzierung bei Nachfragerisiko zu einem anderen Abbauprofil führt, als dies bei einer eınheitlichen Preisgestaltung der Fall wäre.

1) Solow (1977) diskutiert die Marktform des Monopols bei Risiko über Explorationserfolge; Dasgupta/Stiglitz (1980) tun dies bei Rısiko über

2) Pindyck (1980), S.1210. 
3.2.1. Ressourcenabbau bei Antizipation eines riskobehafteten Nachfrageverlaufs

Der Monopolist berücksichtigt in seinem Kalkül den erwarteten Nachfrageverlauf. Für ihn ist der Marktpreis kein Datum, sondern er geht als Varıable einer Preisplanung in Abhängigkeıt von den abgebauten Rohstoffmengen in das Optimierungskalkül ein.

Optimiert wird von dem Monopolisten das Problem

$$
\begin{array}{ll}
\max _{(q(t), T)} & E\left\{U\left[{ }_{0} \int^{T}[p(t) q(t)-C(q(t))] e^{-} \delta t d t\right]\right\} \\
\text { u.d.B. } & R_{0} \geq{ }_{0} T^{T} q(t) d t, R_{0} \text { gegeben, } q(t) \geq 0 \\
& p(t)=N(q(t)) u(t)
\end{array}
$$

Dies führt über die Lagrangefunktion zu den notwendigen Bedingungen

(3.19)(i) $E\left\{U^{\prime}\left[p(t)+q(t) N^{\prime}(q(t)) u(t)-C^{\prime}(q(t))\right]\right\} e^{-\delta t}-\lambda \begin{cases}=0 & q(t)>0 \\ & \text { für } \\ \leq 0 & q(t)=0\end{cases}$

(3.19)(ii) $R_{0}-{ }_{0} \int^{T} q(t) d t$

$$
\left\{\begin{array}{lll}
=0 & \lambda>0 \\
& \text { für } & \\
\geq 0 & \lambda=0
\end{array}\right.
$$

(3.19)(iii) $E\left\{U^{\prime}[p(T) q(T)-C(q(T))]\right\} e^{-\delta T}-\lambda q(T)=0$. 
Der Monopolist plant seine Abbaumengen nun anhand seiner Presserwartungen und seınes Risikoverhaltens. 1)

$$
\left\{E[p(t)]+\operatorname{cov}\left[U^{\prime}, p(t)\right] E\left(U^{\prime}\right)^{-1}\right\}\left[1+n_{e}(t)^{-1}\right]=\lambda e^{\prime} \delta t E\left(U^{\prime}\right)^{-1}+C^{\prime}(q(t))
$$

Im Erwartungsnutzenmaximum bietet der Monopolist jene Abbaumenge an, bei der sein erwarteter Grenzerlös gleich ist der Summe aus Opportunitäts- und Grenzabbaukosten der Ressource plus einer Korrekturgröße für eine bestimmte Risikohaltung. In der marginalen Betrachtung berücksichtigt der risikoaverse (risikofreudige) Monopolist, daß er einen Rückgang (Anstieg) des Grenznutzens erfährt, wenn eine zusätzliche Rohstoffeinheit angeboten wird.

Gleichung 3.19 (i) liefert die zeitliche Entwicklung der Abbaumengen. Die Trennung der Zufallsvariablen $u(t)$ vom systematischen Tell der Nachfrage führt zu

$$
E\left[U^{\prime} u(t)\right]\left[N\left(q(t)+N^{\prime}(q(t)) q(t)\right]-E\left(U^{\prime}\right) C^{\prime}(q(t))=\lambda e^{\delta t}\right.
$$

und mittels der Definition für $\Gamma^{2}$ ) zu

$$
\Gamma^{-1}\left[N\left(q(t)+N^{\prime}(q(t)) q(t)\right]-C^{\prime}(q(t))=\lambda e^{\delta t E\left(U^{\prime}\right)-1 .}\right.
$$

Nun folgt für $d \Gamma / d t=0$

$$
\begin{aligned}
d q / d t= & \delta\left\{\left[N(q(t))+N^{\prime}(q(t)) q(t)\right] \Gamma^{-1}-C^{\prime}(q(t))\right\} \\
& \left\{2 N^{\prime}(q(t)) \Gamma^{-1}-C^{\prime \prime}(q(t))+\lambda e^{\delta} \delta E\left(U^{\prime}\right)-2\left[d E\left(U^{\prime}\right) / d q\right]\right\}-1<0
\end{aligned}
$$

1) Es steht $\eta_{e}(t)$ für die erwartete Preıselastızitàt der Nachfrage $\eta_{e}(t)-1=$

2) $\quad$ Siehe $S .73$. 
und aus der Nachfragefunktıon für die zeitliche Entwicklung der Preiserwartungen

$$
d E[p(t)] / d t=d p / d t=N^{\prime}(q(t))(d q / d t)>0 .
$$

Ein Vergleich der Allokationsprozesse zwischen polypolistischer Angebotsseite (Gleichung 3.11) und monopolistischem Rohstoffangebot (Gleichung 3.21) hat die Annahme zu treffen, daß die Anbieter in beiden Marktformen mit derselben Kostenfunktion einen Ressourcenbestand abbauen, der für das Polypol im Aggregat dem des Monopols entspricht. Die Nachfrage nach Rohstoffen ist identisch, die Diskontraten sind gleich und alle Anbieter zeigen das gleiche risikoaverse Verhalten. Unter diesen Annahmen erhält man auch unter Risiko ein konservierendes Verhalten für den Monopolisten. Im Vergleich zum Polypol verlaufen Preis- und Mengenpfad flacher.

Eine zur Transversalitätsbedingung 2.34 anałoge Aussage wird auch unter Risiko erhalten. 3.19 (iii) erlaubt mit $\mathrm{q}(\mathrm{T})>0$ und 3.20 die Folgerung:

$$
-\left\{E[p(T)]+\operatorname{cov}\left[U^{\prime}, p(T)\right] E\left(U^{\prime}\right)^{-1}\right\} n_{e}(T)^{-1}=C(q(T)) q(T)^{-1}-C^{\prime}(q(T))>0 .
$$

Verglichen mit einem polypolistischen Anbieter baut der Monopolist auch dann ab, wenn die Grenzkosten des Abbaus niedriger als die Durchschnittskosten sind.

\subsubsection{Ressourcenabbau und Preisdifferenzierung bei räumlich} getrennten Märkten mit unterschiedlichem Risiko

Ist der Monopolist in der Lage, einen Markt aufgrund unterschiedlicher Rısiken und unterschiedlicher Nachfrageverläufe in zwei Teilmärkte zu spalten, bzw. liegen diese vor, so wird er versuchen, seinen Erwartungsnutzen durch Preisdifferenzierung zwischen den beıden Märkten zu erhohen. 
Es soll für den Nachfrageverlauf auf den Teilmärkten jeweıls gelten:

(3.24)(i) $\quad p_{1}(t)=N_{1}\left(q_{1}(t)\right) u_{1}(t)$ mit d $N_{1} / d q_{1}=N_{1}{ }^{\prime}<0, d 2 N_{1} / d q_{1} 2=0$

(3.24)(ii) $\quad p_{2}(t)=N_{2}\left(q_{2}(t)\right) u_{2}(t) m i t d N_{2} / d q_{2}=N_{2}^{\prime}<0, d 2 N_{2} / d q_{2}{ }^{2}=0$

$u_{1}(t), u_{2}(t)$ seien über die Zeit unabhängig und identisch verteilte Zufallsvariable mit $E\left[u_{1}(t)\right]=E\left[u_{2}(t)\right]=1, \operatorname{var}\left[u_{1}(t)\right]=\sigma_{1} 2, \operatorname{var}\left[u_{2}(t)\right]=\sigma_{2}{ }^{2}$ und $\operatorname{cov}\left[\mathbf{u}_{1}(t), \mathbf{u}_{2}(t)\right]=0$.

Weiter soll für das Verhältnis der erwarteten choke-off Preise und die Nachfrageverläufe gelten: $N_{1}(0)>N_{2}(0)$ und $N_{1}{ }^{\prime}\left(q_{1}(t)\right)<N^{\prime}{ }_{2}\left(q_{2}(t)\right)$.

Für den nun preisdifferenzierenden Monopolisten lautet die Problemstellung: 1)

$$
\begin{array}{ll}
\max _{\left(q_{1}(t), q_{2}(t), T\right)} & E\left\{\left[U_{0} \int^{T}\left[p_{1}(t) q_{1}(t)+p_{2}(t) q_{2}(t)-C(q(t))\right] e-\delta t d t\right]\right\} \\
\text { u.d.B. } & R_{0} \geq{ }_{0} \int^{T} q(t) d t, R_{0} \text { gegeben, } q_{1}(t), q_{2}(t) \geq 0 \\
& p_{j}(t)=N_{j}\left(q_{j}(t)\right) u_{j}(t), j=1,2
\end{array}
$$

Dies führt zu den notwendigen Bedingungen, $\mathrm{j}=1,2$

(3.26)(i) $E\left\{U^{\prime}\left[p_{j}(t)+q_{j}(t) N_{j}{ }^{\prime}\left(q_{j}(t)\right) u_{j}(t)-C^{\prime}(q(t))\right]\right\} e^{-\delta t}-\lambda \begin{cases}=0 & q(t)>0 \\ & \text { für } \\ \leq 0 & q(t)=0\end{cases}$

(3.26)(ii) $\quad R_{0}-{ }_{0} \int^{\top} q(t) d t$

$$
\left\{\begin{array}{lll}
=0 & \lambda>0 \\
& \text { für } & \\
\geq 0 & \lambda & =0
\end{array}\right.
$$

1) Zum statischen Fall einer Preısdifferenzierung bei Risıko siehe bspw. Blair/Heggestad (1977). 
(3.26)(iii) $E\left\{U^{\prime}\left[p_{1}(T) q_{1}(T)+p_{2}(T) q_{2}(T)-C(q(T))\right]\right\} e^{-\delta T}-\lambda q(T)=0$

und liefert für die erwarteten gleichgewichtigen Marktpreise in Abhängigkeit von den Abbaumengen die Beziehungen

$$
\begin{array}{r}
\left\{E\left[p_{j}(t)\right]+\operatorname{cov}\left[U^{\prime}, p_{j}(t)\right] E\left(U^{\prime}\right)-1\right\}\left[1+n_{e}(t)_{j}{ }^{-1}\right]=\lambda e^{\delta t E\left(U^{\prime}\right)-1}+C^{\prime}(q(t)), \\
j=1,2 .
\end{array}
$$

Was für den Monopolisten ohne Preisdifferenzierung galt, gilt nun für jeden der beiden Teilmärkte bei Preisdifferenzierung. Der um eine Risikokomponente korrigierte erwartete Grenzerlös ist gleich den Opportunitätskosten der Ressourcennutzung plus den Grenzkosten des Abbaus.

Solange auf beiden Märkten angeboten wird, gilt die Gleichheit der korrigierten Grenzerlöse

$$
\begin{aligned}
& \left.\left\{E\left[p_{1}(t)\right]+\operatorname{cov}\left[U^{\prime}, p_{1}(t)\right] E\left(U^{\prime}\right)^{-1}\right\}\left[1+n_{e}(t)\right)_{1}^{-1}\right]= \\
& \left\{E\left[p_{2}(t)\right]+\operatorname{cov}\left[U^{\prime}, p_{2}(t)\right] E\left(U^{\prime}\right)^{-1}\right\}\left[1+n_{e}(t) 2^{-1}\right] .
\end{aligned}
$$

Die Relation der sich einstellenden Preise auf beiden Teilmärkten ist nun nicht mehr nur von der (erwarteten) Preiselastizität der Nachfrage abhängig, 1) sondern auch von dem Risikoverhalten des Monopolisten gegenüber den beiden Teilmärkten.

Zeigt der Monopolist gegenüber beiden Teilmärkten die gleiche Risikoaversion $\operatorname{cov}\left[\mathbf{U}^{\prime}, \mathbf{p}_{1}(\mathrm{t})\right]=\operatorname{cov}\left[\mathrm{U}^{\prime}, \mathbf{p}_{2}(\mathrm{t})\right]<0$, so gilt die Aussage

$$
E\left[p_{1}(t)\right]><E\left[p_{2}(t)\right] \Leftrightarrow \eta_{e}(t)_{1}><n_{e}(t)_{2} .
$$

Bei identischem Risikoverhalten oder gleichem Risiko auf den Teilmärkten wird dort eine höhere Preiserwartung durchzusetzen versucht, wo die erwartete Preiselastizität der Nachfrage dem Betrage nach kleiner ist.

1) Unter Sicherheit galt $p_{1}(t)><p_{2}(t) \Leftrightarrow \eta_{1}(t)><\eta_{2}(t)$, für $n_{1}(t), n_{2}(t)<-1$; vgl. Gleichung 2.44 . 
Diese Zuordnung ist im allgemeinen nicht mehr möglich, wenn der Monopolist ein unterschiedliches Risikoverhalten für jeden Teilmarkt zeigt: Sei er bspw. auf Markt 1 risikoaverser als auf Markt $2\left(\operatorname{cov}\left[U^{\prime}, p_{1}(t)\right]<\right.$ $\left.\operatorname{cov}\left[U^{\prime}, p_{2}(t)\right]<0\right)$, dann kann bei $\eta_{e}(t)_{1}<\eta_{e}(t)_{2}$ sowohl $E\left[p_{1}(t)\right]>E\left[p_{2}(t)\right]$ als auch $E\left[p_{1}(t)\right]>E\left[p_{2}(t)\right]$ gelten.

Aufgrund unterschiedlichen Risikoverhaltens werden nun auch die erwarteten Preispfade beider Märkte eine unterschiedliche Steigung aufweisen.

Aus 3.26 (i) folgt mittels der Definitionen

$$
\Gamma_{j}=E\left[p_{j}(t)\right]\left\{E\left[p_{j}(t)\right]+\operatorname{cov}\left[U^{\prime}, p_{j}(t)\right] E\left(U^{\prime}\right)-1\right\}-1, j=1,2
$$

die zu 3.27 äquivalente Schreibweise

$$
\Gamma_{j^{-1}}\left[N_{j}\left(q_{j}(t)\right)+N_{j}\left(q_{j}(t)\right) q_{j}(t)\right]-C^{\prime}(q(t))=\lambda e^{\delta t E\left(U^{\prime}\right)^{-1}, j=1,2 .}
$$

Daraus folgt für die Relationen der zeitlichen Entwicklung der Handelsmengen auf den beiden Teilmärkten $\left(d \Gamma_{j} / d t=0, j=1,2\right)$

$$
\Gamma_{2} N_{1}{ }^{\prime}\left(q_{1}(t)\right)\left(d q_{1} / d t\right)=\Gamma_{1} N_{2}{ }^{\prime}\left(q_{2}(t)\right)\left(d q_{2} / d t\right) .
$$

Hierin steht $N_{j}{ }^{\prime}\left(q_{j}(t)\right)\left(d q_{j} / d t\right)$ für $d E[p(t)] / d t$ und man sieht, daß die erwartete Preisentwicklung nur dann auf beiden Märkten gleich ist, wenn gegenüber beiden Märkten das gleiche Risikoverhalten gezeigt wird.

Die notwendigen Bedingungen 3.27' $(j=1,2)$ liefern

$$
\begin{aligned}
& \left\{2 N_{1}{ }^{\prime}\left(q_{1}(t)\right) \Gamma_{1}{ }^{-1}-C^{\prime \prime}(q(t))+\lambda e^{\delta t E\left(U^{\prime}\right)-1}\left[d E\left(U^{\prime}\right) / d q_{1}\right]\right\}\left(d q_{1} / d t\right)+
\end{aligned}
$$

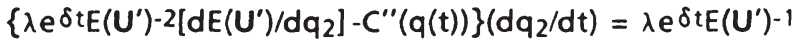

(3.31)(ii) $\left\{2 \mathrm{~N}_{2}{ }^{\prime}\left(\mathrm{q}_{2}(\mathrm{t})\right) \Gamma_{2}-^{-1}-\mathrm{C}^{\prime \prime}(\mathrm{q}(\mathrm{t}))+\lambda \mathrm{e}^{\delta t E\left(\mathrm{U}^{\prime}\right)-1}\left[\mathrm{dE}\left(\mathrm{U}^{\prime}\right) / \mathrm{dq} \mathrm{q}_{2}\right]\right\}\left(\mathrm{dq} \mathrm{q}_{2} / \mathrm{dt}\right)+$

$$
\left\{\lambda e^{\delta t E}\left(U^{\prime}\right)-2\left[d E\left(U^{\prime}\right) / d q_{1}\right]-C^{\prime \prime}(q(t))\right\}\left(d q_{1} / d t\right)=\lambda e^{\delta t E}\left(U^{\prime}\right)-1 .
$$

Zusammen mit 3.30 folgt

(3.32)(i) $\quad \mathrm{dq}_{1} / \mathrm{dt}=\delta \lambda \mathrm{e}^{\delta \mathrm{tE}}\left(\mathrm{U}^{\prime}\right)^{-1} \Gamma_{1} \mathrm{~N}_{2}{ }^{\prime}\left(\mathrm{q}_{2}(\mathrm{t})\right) \Lambda^{-1}<0$ 


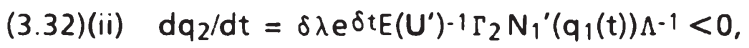

mit $\Lambda=2 N_{1}{ }^{\prime}\left(q_{1}(t)\right) N_{2}{ }^{\prime}\left(q_{2}(t)\right)+\left\{\lambda e^{\delta t E}\left(U^{\prime}\right)-2\left[d E\left(U^{\prime}\right) / d q_{1}\right]-C^{\prime \prime}(q(t))\right\}$

$$
\left.\left[\Gamma_{1} N_{2}{ }^{\prime}\left(q_{2}(t)\right)+\Gamma_{2} N_{1}{ }^{\prime}\left(q_{1}(t)\right)\right]>0.1\right)
$$

Es ergibt sich im Vergleich

$$
d q_{1} / d t><q_{2} / d t \Leftrightarrow \Gamma_{1} N_{2}{ }^{\prime}\left(q_{2}(t)\right)><\Gamma_{2} N_{1}{ }^{\prime}\left(q_{1}(t)\right) .
$$

Für identisches Risikoverhalten gegenüber den Teilmärkten erhält man die eindeutige Aussage, daß aufgrund des unelastischer unterstellten Nachfrageverlaufs auf Markt 1 die Handelsmengen auf diesem Markt eine geringere zeitliche Veränderung aufweisen als auf dem Markt 2. Mit dieser Aussage wurde auch der Vergleich der Entwicklung der Handelsmengen unter Sicherheit abgeschlossen. Gegenüber dem Allokationsmuster eines risikoneutralen Monopolisten bei Preisdifferenzierung kann für den risikoaversen Monopolisten wieder ein Allokationsbias zugunsten der Zukunft abgeleitet werden.

Gibt man andererseits gleiche Nachfrageverläufe auf beiden Teilmärkten vor, dann wählt der preisdifferenzierende Monopolist auf demjenigen Teilmarkt ein flacheres zettliches Handelsprofil, auf dem das Risiko geringer ist:

$$
\mathrm{dq}_{1} / \mathrm{dt}><\mathrm{dq}_{2} / \mathrm{dt} \Leftrightarrow \Gamma_{2}><\Gamma_{1} .
$$

D.h. der Abbauunternehmer plant, auf jenem Markt länger anzubieten, auf dem das Risiko geringer ist.

1) Esist $d E\left(U^{\prime}\right) / d q_{1}=d E\left(U^{\prime}\right) / d q_{2}$. 
In 3.33 ist zu sehen, daß trotz unterschiedlicher Risiken auf beiden Märkten und unterschiedlıcher Nachfrageverläufe für beide Märkte das gleiche Handelsprofil realisiert werden kann. Bspw. ist dies der Fall, wenn ein auf dem Teilmarkt 1 höheres Risiko $\left(\Gamma_{1}>\Gamma_{2}\right)$ durch einen entsprechend unelastischeren Nachfrageverlauf auf diesem Teilmarkt $\left(N_{1}{ }^{\prime}\left(q_{1}(t)\right)<N_{2}{ }^{\prime}\left(q_{2}(t)\right)\right)$ "kompensiert" wird.

Die Gesamtausbringung bei Preisdifferenzierung bzw. das Abbauprofil ergibt sich aus $\mathrm{dq} / \mathrm{dt}=\mathrm{dq} \mathrm{q}_{1} / \mathrm{dt}+\mathrm{dq} \mathrm{q}_{2} / \mathrm{dt}$ mit $3.32 \mathrm{zu}$

$$
(d q / d t)_{d}=\delta \lambda e^{\delta t E\left(U^{\prime}\right)-1}\left[\Gamma_{1} N_{2}{ }^{\prime}\left(q_{2}(t)\right)+\Gamma_{2} N_{1}{ }^{\prime}\left(q_{1}(t)\right)\right] \Lambda^{-1} .
$$

Ohne Preisdifferenzierung, mit der erwarteten Gesamtnachfrage $E[p((t)]=N(q(t))$ als Summe der erwarteten Nachfragen auf den beiden Teilmärkten, baut der Monopolist ab gemäß

$$
\begin{aligned}
(d q / d t)_{0}= & \delta \lambda e^{\delta t E\left(U^{\prime}\right)-1 .} \\
& \left\{2 N^{\prime}(q(t)) \Gamma^{-1}-C^{\prime \prime}(q(t))+\lambda e^{\left.\delta t E\left(U^{\prime}\right)-2\left[d E\left(U^{\prime}\right) / d q\right]\right\}-1 .} .\right.
\end{aligned}
$$

Da für die erwartete Gesamtnachfrage gilt

$$
N^{\prime}(q(t))=N_{1}{ }^{\prime}\left(q_{1}(t)\right) N_{2}{ }^{\prime}\left(q_{2}(t)\right)\left[N_{1}{ }^{\prime}\left(q_{1}(t)\right)+N_{2}{ }^{\prime}\left(q_{2}(t)\right)\right]^{-1},
$$

erhält man

$$
(d q / d t)_{\circ}=\delta \lambda e^{\delta t E}\left(U^{\prime}\right)-1 \Gamma\left[N_{1}{ }^{\prime}\left(q_{1}(t)\right)+N_{2}{ }^{\prime}\left(q_{2}(t)\right)\right] \theta-1 .
$$

wobei

$$
\begin{aligned}
\theta & =2 N_{1}{ }^{\prime}\left(q_{1}(t)\right) N_{2}^{\prime}\left(q_{2}(t)\right)+ \\
& \left\{\lambda e^{\delta t E\left(U^{\prime}\right)-2\left[d E\left(U^{\prime}\right) / d q\right]}-C^{\prime \prime}(q(t))\right\} \Gamma\left[N_{1}^{\prime}\left(q_{1}(t)\right)+N_{2}^{\prime}\left(q_{2}(t)\right)\right]>0 .
\end{aligned}
$$

Ein Vergleich von 3.35' mit 3.34 zeigt, daß nun die Abbauprofile mit und ohne Preisdifferenzierung im allgemeinen voneinander abweichen werden.

Preisdifferenzierung wirkt genau dann konservierend auf die Ressourcennutzung $\left[0>(d q / d t)_{d}>(d q / d t)_{0}\right]$, wenn das Risıko des Gesamtmarktes $\Gamma$ 
großer ist als die Summe der mit Nachfrageelementen gewichteten Risıken der Tellmarkte $\Gamma_{1}, \Gamma_{2}$. Mit anderen Worten: Der risikoaverse Abbauunternehmer erschopft seınen Ressourcenbestand im Falle der Preisdifferenzierung spater, wenn er mittels der Preisdifferenzierung seın Risıko senkt. Oder umgekehrt: Der risikoaverse Abbauunternehmer erschöpft seinen Ressourcenbestand im Falle der Preisdifferenzierung früher [ $(\mathrm{dq} / \mathrm{dt})_{d}<$ $\left.(d q / d t)_{0}<0\right]$, wenn er mittels der Preisdifferenzierung sein Risiko erhöht:

$$
(d q / d t)_{d}><(d q / d t)_{0} \Leftrightarrow \Gamma_{1} \xi_{2}+\Gamma_{2} \xi_{1}><\Gamma ;
$$

dabel ist $\quad \xi_{1}=N_{1}{ }^{\prime}\left(q_{2}(t)\right)\left[N_{1}{ }^{\prime}\left(q_{1}(t)\right)+N_{2}{ }^{\prime}\left(q_{2}(t)\right)\right]^{-1}, i=1,2$. 


\subsection{Das Oligopol: Mengenkonkurrenz im homogenen Duopol}

Neben dem schon erwähnten Beitrag Dasgupta/St'glitz (1980b) existieren in der Ressourcentheorie keine Modellbetrachtungen uber Oligopolsituationen unter Nachfragerisiko. Dasgupta/Stiglitz diskutieren die intertemporale Allokation in einer Duopolsituation bei Risiko über den Zeitpunkt des Aufkommens eines Ressourcensubstitutes.

Die in Abschnitt 2.3. unter Sicherheit durchgeführte Analyse soll hier unter Modellierung eines bestimmten Risikoverhaltens fortgesetzt werden. Es gelten für die Angebotsseite die Annahmen des Abschnitts 2.3. mit dem Zusatz, daß jeder der beiden Duopolisten eine Nutzenfunktion (Risikopräferenzfunktion) $U_{i}\left(G_{i}\right)$ besitze, mit $U_{1}{ }^{\prime}>0$ und $U_{i}{ }^{\prime \prime}<0$. Der Erwartungsnutzen über den gesamten diskontierten Periodengewinnen bilde das Zielfunktional der Duopolisten.

Die Abbaukosten seien identisch. Beiden Duopolisten stehen die gleichen Informationen zur Verfügung. Beide Duopolisten besitzen rationale Erwartungen über die Preise auf den zukünftigen Kassamärkten. Aufgrund ihrer Preiserwartungen planen sie, ihre Ressourcenbestände in bestimmter Weise zu erschöpfen.

Für die Nachfrageseite stehe die in 3.10 eingeführte Nachfragefunktion. Im Erwartungsnutzenkonzept lauten die Optimierungsprobleme der Duopolisten, $i=1,2$

$$
\begin{array}{ll}
\max _{\left(q_{1}(t), T_{i}\right)} & E\left\{U_{i}\left[{ }_{0} J^{\top},\left[p(t) q_{1}(t)-c q_{1}(t)\right] e^{-\delta t d t}\right]\right\} \\
\text { u.d.B. } & R_{0_{1}} \geq{ }_{0} \int^{\top} q_{1}(t) d t, R_{01} g e g e b e n, q_{1}(t) \geq 0 \\
& p(t)=N(q(t)) u(t), \text { mit } q(t)=q_{1}(t)+q_{2}(t) .
\end{array}
$$

Diese besitzen die notwendigen Bedingungen 
(3.37)(i) $E\left\{U^{\prime}\left[p(t)+q_{1}(t) N^{\prime}(q(t)) u(t)-c\right]\right\} e^{-\delta t-\lambda_{1}} \begin{cases}=0 & q_{i}(t)>0 \\ & \text { für } \\ \leq 0 & q_{i}(t)=0\end{cases}$

(3.37)(ii) $R_{01}-{ }_{10} f^{\top}: q_{i}(t) d t$

$$
\left\{\begin{array}{lll}
=0 & \lambda_{1}>0 \\
& \text { für } & \\
\geq 0 & \lambda_{i}=0
\end{array}\right.
$$

(3.37)(iii) $E\left\{U^{\prime},\left[p\left(T_{1}\right)-c\right]\right\} q_{i}\left(T_{1}\right) e^{-\delta} T_{1}-\lambda_{i} q_{i}\left(T_{i}\right)=0$.

Es folgen ähnliche Ergebnisse wie im Monopolfall (vgl. 3.20), nun allerdings unter Beachtung der Marktanteile der Duopolisten $\alpha_{i}(t)=q_{i}(t) / q(t)$, $i=1,2.11$

$$
\begin{aligned}
& E[p(t)]\left[1+\alpha_{i}(t) n_{e}(t)_{i}-1\right]=\lambda_{i} e^{\delta t E}\left(U^{\prime}{ }_{i}\right)^{-1}+c- \\
& {\left[1+\alpha_{i}(t) n_{e}(t)_{i}{ }^{-1}\right] \operatorname{cov}\left[U^{\prime}{ }_{i}, p(t)\right] E\left(U^{\prime}{ }_{i}\right)^{-1} . }
\end{aligned}
$$

Da beide Duopolisten auf die gleiche Art aus derselben Informationsmenge ihre Preiserwartungen bilden, werden sich diese nicht unterscheiden. Unterschiedlich kann jedoch das Risikoverhalten sein.

Wird Risikoaversion unterstellt, so kann auch unter der Marktform des Angebotsduopols die konservierende Wirkung des risikoaversen Verhaltens abgeleitet werden (Gleichung 3.28). Verglichen mit einem risikoneutralen Duopolisten baut der risikoaverse Duopolist ceteris paribus aufgrund seiner Risıkoscheue in gegenwartsnäheren Perioden kleinere Mengen ab, um in spateren Perioden mit größeren Fördermengen als im Falle der Risikoneutralität abzubauen. Der Abbaupfad zeigt einen flacheren Verlauf.

1) $n_{e}(t)$ steht wieder für die erwartete Preiselastizität der Nachfrage. 
Es folgt aus 3.37 (i) über die Differentiation nach der Zeit und mit

$$
\Gamma_{1}=E[p(t)]\left\{E[p(t)]+\operatorname{cov}\left[U^{\prime}{ }_{1}, p(t)\right] E\left(U_{1}^{\prime}\right)^{-1}\right\}-1
$$

für jeden Duopolisten:

(3.39)(i) $\quad d q_{1} / d t=\delta e \delta t\left\{N^{\prime}\left(q(t)\left[2 \lambda_{1} \Gamma_{1} E\left(U^{\prime}{ }_{1}\right)^{-1}-\lambda_{2} \Gamma_{2} E\left(U^{\prime}{ }_{2}\right)^{-1}\right]+\right.\right.$

$$
\left.\left[\lambda_{1} \Gamma_{1} E\left(U^{\prime}{ }_{1}\right)^{-1}\right]\left[\lambda_{2} \mathrm{e}^{\delta} \delta \Gamma_{2} E\left(U^{\prime}{ }_{2}\right)^{-2}\right]\left[d E\left(U^{\prime}{ }_{2}\right) / d q_{2}\right]\right\} A(t)-1<0
$$

(3.39)(ii) $d q_{2} / d t=\delta e^{\delta t}\left\{N^{\prime}\left(q(t)\left[2 \lambda_{2} \Gamma_{2} E\left(U^{\prime}\right)^{-1}-\lambda_{1} \Gamma_{1} E\left(U^{\prime}{ }_{1}\right)^{-1}\right]+\right.\right.$

$$
\left.\left[\lambda_{2} \Gamma_{2} E\left(U^{\prime}{ }_{2}\right)^{-1}\right]\left[\lambda_{1} e^{\delta t} \Gamma_{1} E\left(U^{\prime}{ }_{1}\right)^{-2}\right]\left[d E\left(U^{\prime}{ }_{1}\right) / d q_{1}\right]\right\} A(t)-1<0 \text {. }
$$

mit

$$
\begin{aligned}
& \left.A(t)=3 N^{\prime}(q(t))\right)^{2}+2 N^{\prime}(q(t)) e^{\delta t}\left\{\lambda_{1} \Gamma_{1} E\left(U^{\prime}{ }_{1}\right)-2\left[d E\left(U^{\prime}{ }_{1}\right) / d q_{1}\right]+\right. \\
& \left.\left[\lambda_{2} \Gamma_{2} E\left(U^{\prime}{ }_{2}\right)-2\right]\left[d E\left(U^{\prime}{ }_{2}\right) / d q_{2}\right]\right\}+\Gamma_{1} \Gamma_{2}\left[\delta \lambda_{1} e^{\delta t E(}\left(U^{\prime}{ }_{1}\right)-2\right]\left[d E\left(U^{\prime}{ }_{1}\right) / d q_{1}\right] \\
& {\left[\delta \lambda_{2} e^{\delta t} \Gamma_{2} E\left(U^{\prime}{ }_{2}\right)-2\right]\left[d E\left(U^{\prime}{ }_{2}\right) / d q_{2}\right]>0 .}
\end{aligned}
$$

Der gesamte Ressourcenbestand $R_{0}=R_{01}+R_{02}$ wird abgebaut entlang des Pfades

$$
\begin{aligned}
d q / d t= & \delta e^{\delta t}\left\{N ^ { \prime } \left(q(t)\left[\lambda_{1} \Gamma_{1} E\left(U^{\prime}{ }_{1}\right)^{-1}+\lambda_{2} \Gamma_{2} E\left(U^{\prime}{ }_{2}\right)^{-1}\right]+\right.\right. \\
& {\left[\lambda_{1} \Gamma_{1} E\left(U^{\prime}{ }_{1}\right)^{-1}\right]\left[\lambda_{2} \Gamma_{2} E\left(U^{\prime}{ }_{2}\right)^{-1}\right]\left\{\left[e^{\delta t E} E\left(U^{\prime}{ }_{1}\right)^{-1}\right]\left[d E\left(U^{\prime}{ }_{1}\right) / d q_{1}\right]+\right.} \\
& {\left.\left[e^{\delta t E}\left(U^{\prime}{ }_{2}\right)^{-1}\right]\left[d E\left(U^{\prime}{ }_{2}\right) / d q_{2}\right]\right\} A(t)^{-1}<0 . }
\end{aligned}
$$

Eine Aussage, wie sie auf Seite $\mathbf{4 8}$ über den Zusammenhang zwischen Schattenpreis und Ressourcenbestand bzw. zwischen Ressourcenbestand und Erschöpfungszeitpunkt abgeleitet wurde, ist nun nicht mehr möglich. Die zu 2.53 äquivalente Gleichung lautet unter Risiko 


$$
\begin{gathered}
\left(\Gamma_{2}-\Gamma_{1}\right) N(q(t))+\left[\Gamma_{2} q_{1}(t)-\Gamma_{1} q_{2}(t)\right] N^{\prime}(q(t))= \\
\Gamma_{1} \Gamma_{2} e^{\delta t}\left[\lambda_{1} E\left(U^{\prime}{ }_{1}\right)^{-1}-\lambda_{2} E\left(U^{\prime}\right)^{-1}\right] .
\end{gathered}
$$

Hohere Nutzungskosten bel Duopolist $1\left[\lambda_{1} E\left(U^{\prime}{ }_{1}\right)^{-1}>\lambda_{2} E\left(U^{\prime}{ }_{2}\right)^{-1}\right]$ können nun durchaus zu eıner größeren Abbaumenge als beı Duopolist 2 führen $\left[q_{1}(t)>q_{2}(t)\right]$, wenn der Duopolist 2 risikoaverser ist $\left(\Gamma_{2}>\Gamma_{1}>1\right)$.

Zu eıner eindeutıgen Aussage kommt man dann wieder, wenn gleiches Risikoverhalten für beide Duopolısten unterstellt wird $\left(\Gamma_{1}=\Gamma_{2}=\Gamma\right)$. Gleichung 3.41 wird dann zu

$$
\left[q_{1}(t)-q_{2}(t)\right] N^{\prime}(q(t))=\Gamma e^{\delta t}\left[\lambda_{1} E\left(U^{\prime}{ }_{1}\right)^{-1}-\lambda_{2} E\left(U^{\prime}{ }_{2}\right)^{-1}\right]
$$

und es können wieder die Aussagen abgeleitet werden, wie sie in 2.53 möglich sind und dargestellt wurden.

Für weitere Aussagen sei neben der Annahme gleichen Risikoverhaltens noch eine zusätzliche Symmetrieannahme bezüglich der Reservenbestände gemacht: $R_{01}=R_{02}$. Als Folge davon wird $\lambda_{1}=\lambda_{2}=\lambda$ werden. Die Gleichungen 3.39 und 3.40 vereinfachen sich zu

$$
\begin{aligned}
& d q_{1} / d t=\delta \lambda e^{\delta t E}\left(U^{\prime}\right)-1\left\{N^{\prime}\left(q(t) \Gamma^{-1}+\left[\lambda e^{\delta t E}\left(U^{\prime}\right)-2\right]\left[d E\left(U^{\prime}\right) / d q_{1}\right]\right\} B(t)^{-1}\right. \\
& d q_{1} / d t<0, i=1,2 \\
& d q / d t=d q_{1} / d t+d q_{2} / d t \text { mit } d q_{1} / d t=d q_{2} / d t \text { und } \\
& B(t)=\left\{N^{\prime}\left(q(t) \Gamma^{-1}+\left[\lambda e^{\delta t E}\left(U^{\prime}\right)-2\right]\left[d E\left(U^{\prime}\right) / d q_{i}\right]\right\}^{2}+2\left[N^{\prime}\left(q(t) \Gamma^{-1}\right] 2>0 .\right.\right.
\end{aligned}
$$

Dies gestattet nun den Vergleich 1) mit den Marktformen des Polypols bzw. des Monopols. Unter der vereinfachenden Annahme konstanter durch-

1) Der Vergleich geht aus von gleichen Diskontraten, gleichen Abbaukosten, gleichem Nachfrageverlauf, gleichem Risikoverhalten und gleichen Ressourcenbeständen für die unterschiedlichen Marktformen. Es bezeichnen die Indices P, M, D die zu den Marktformen des Polypols, des Monopols und des Duopols zugehörigen Variablen. 
schnittlicher Abbaukosten werden die Gleichungen 3.11 und $3.21 \mathrm{zu}$

$$
(d q / d t)_{p}=\delta \lambda p e^{\delta t} E\left(U^{\prime}\right)^{-1}\left\{N^{\prime}(q(t)) \Gamma^{-1}+\lambda p e^{\delta t E}\left(U^{\prime}\right)-2\left[d E\left(U^{\prime}\right) / d q\right]\right\}-1
$$

und

$$
\begin{aligned}
(d q / d t)_{M}=\delta \lambda_{M} e^{\delta t} E\left(U^{\prime}\right)-1 . & \\
& \left\{2 N^{\prime}(q(t)) \Gamma^{-1}+\lambda_{M} e^{\left.\delta t E\left(U^{\prime}\right)-2\left[d E\left(U^{\prime}\right) / d q\right]\right\}-1 .}\right.
\end{aligned}
$$

Der Abbau in der Duopolsituation entwickelt sich nach

$$
\begin{gathered}
(d q / d t)_{D}=\delta \lambda_{D} e^{\delta t E}\left(U^{\prime}\right)-12\left\{N^{\prime}\left(q(t) \Gamma^{-1}+\left[\lambda_{D} e^{\delta t E}\left(U^{\prime}\right)-2\right]\left[d E\left(U^{\prime}\right) / d q_{1}\right]\right\}\right. \\
\left\{\left\{N^{\prime}\left(q(t) \Gamma^{-1}+\left[\lambda e^{\delta t E}\left(U^{\prime}\right)-2\right]\left[d E\left(U^{\prime}\right) / d q_{1}\right]\right\} 2+2\left[N^{\prime}\left(q(t) \Gamma^{-1}\right] 2\right\}-1 .\right.\right.
\end{gathered}
$$

Unterstellt wird für den Vergleich, $\operatorname{da} \beta\left[\mathrm{dE}\left(\mathrm{U}^{\prime}\right) / \mathrm{dq}\right] \mathrm{E}\left(\mathrm{U}^{\prime}\right)-1$ in den Marktformen des Polypols und des Monopols bzw. $\left[\mathrm{dE}\left(U^{\prime}\right) / d q_{i}\right] E\left(U^{\prime}\right)-1$ für $I=1,2$ beim Duopol vernachlässigbar klein sei.

Für die Nutzungskosten der Ressourcen unter den drei Marktformen werden dann die folgenden unterschiedlichen Werte erhalten:

$$
\begin{aligned}
& \lambda_{p e} \delta t E\left(U^{\prime}\right)^{-1}=N^{\prime}(q(t)) \Gamma^{-1}-c, \\
& \lambda_{M} e^{\delta t} E\left(U^{\prime}\right)-1=\left[N(q(t))+N^{\prime}(q(t)) q(t)\right] \Gamma^{-1}-c, \\
& \lambda_{D} e^{\delta t} E\left(U^{\prime}\right)^{-1}=\left[N(q(t))+N^{\prime}(q(t)) q(t) / 2\right] \Gamma^{-1}-c .
\end{aligned}
$$

Bei identischen Preiserwartungen $N(q(t))$ für einen bestimmten Zeitpunkt sind die Nutzungskosten unter der Marktform des Polypols größer als im Duopol und die Nutzungskosten im Duopol größer als im Monopol.

Für die Relationen der zeitlichen Entwicklungen der Abbaumengen und der erwarteten Preise folgt auch unter Risıko, daß die Marktform des Duopols hinsichtlich des Allokationsmusters zwischen den Marktformen des Monopols und des Polypols angesiedelt ist. 


\section{Institutionalisierte Substitute für fehlende Zukunftsmärkte}

Zukunftsmärkte geben den Wirtschaftssubjekten vollständige Informatıonen über zukünftige Knappheiten. Für die intertemporale Allokation nichterneuerbarer Naturressourcen steht realiter weder ein vollständiges System von Zukunftsmärkten zur Verfügung, noch sind die exıstierenden Zukunftsmärkte vollkommen. Daraus resultierende Informationsmängel über zukünftige Knappheiten versuchen die Wirtschaftssubjekte durch die Bildung von Erwartungen auszugleichen .

Neben der Entfaltung von Informationsaktivitäten und der Wahl geeıgneter Entscheidungsverfahren haben sich weitere Möglichkeiten zur Minderung oder gar Beseitigung von Risiko als Reaktion auf fehlende Zukunftsmäkte herausgebildet.

Für den Rohstoffhandel sind dies

- langfristige Lieferverträge

- Terminkontrakte

- Optionskontrakte.

Im Bereich des Reservenhandels findet man

- Märkte

- Auktionen

- diskretionäre Vergaben

- Verhandlungen.

Dieses Kapitel betrachtet nacheinander diese Institutionen zunächst für den Rohstoffhandel und dann für den Reservenhandel. 


\subsection{Substitutive Allokationsmechanismen für Rohstoffe}

Beim Rohstoffhandel ist zu unterscheiden zwischen Kassamärkten, Terminmärkten, Terminkontraktmärkten, Optionsmärkten und dem Handel über langfristige Lieferverträge. Bevor auf die langfristıgen Liefervertràge ım Detail eingegangen wird, werden die genannten Märkte in definitorischer Weise gegenübergestellt.

\subsubsection{Kassamarkt, Terminmarkt, Terminkontraktmarkt, Optionsmarkt, langfristige Lieferverträge}

Kassamarkt (Spot-Market):

Auf dem Kassamarkt werden Güter aufgrund persönlicher Vereinbarungen gehandelt. Leistung und Gegenleistung erfolgen in direktem Austausch so, daß der Handel unverzüglich zur Erfüllung kommt. Man kann hier von "güterbezogenem Direktgeschäft" sprechen.1)

Terminmarkt (Forward-Market):

Gegenüber einem Kassageschäft ist ein Termingeschäft dadurch abzugrenzen, daß der Abschluß des Handels und die Erfüllung des Handels zeitlich auseinanderfallen. Leistung und Gegenleistung können zum selben Zeitpunkt erfolgen. Da auch hier unmittelbar das Gut Handelsgegenstand ist,

1) Vgl. Streit (1979b), S.3. Streit spricht hier von einem "warenbezogenen Dırektgeschäft". Der Begiff "Ware" beschränkt sich jedoch auf Sachgüter und ist damit enger gefaßt. 
sind Termingeschäfte als "guiterbezogene Vorausgeschäfte" zu kennzeichnen. 1)

Sowohl der Kassa-als auch der Termınhandel konnen auf organisierten oder nıchtorganısıerten Märkten stattfinden. Auf nicht oder nur wenig organisierten Märkten bleibt der Handel individuell gestaltbar. Eine Annullierung des Handels ist nur zwischen den selben Partnern möglich. Mit zunehmendem Organısatıonsgrad2) eines Terminmarktes wird dieser immer wenıger unterscheidbar von der Institution eines Terminkontraktmarktes.3) Was letztlich als Unterscheidungsmerkmal noch bleibt, ist der unmittelbare Güterbezug des Termingeschäftes.

Terminkontraktmarkt (Futures-Market):4)

Der Terminkontraktmarkt findet nur an Börsen statt. Handelsgegenstand ist nun nicht mehr unmittelbar das Gut, sondern ein standardisierter Vertrag. Die von der Börse vorgenommene Standardisierung bezieht sich dabei auf die fünf Merkmale Quantität, Qualität, Preis, Ort und Zeit der Lieferung eines Gutes. Je nach Gut existieren unterschiedlich viele Standards pro Merkmal.

Für den Käufer bzw. Verkäufer eines Termınkontraktes übernimmt die Abrechnungsstelle der Börse die Marktgegenseite. Die Annullierung des Kaufes bzw. Verkaufes eines Terminkontraktes ist jederzeıt durch den Verkauf bzw. Kauf eines gleichwertigen Terminkontraktes möglich.

1) Vgl. Streit (1979b), S. 5.

2) Zu den Eigenschaften eines organisierten Marktes siehe bspw. Telser/Higınbotham (1977), S.972-974.

3) Terminkontraktmärkte sind historisch aus Terminmärkten entstanden, deren Organisationsgrad immer mehr zunahm.

4) Der Begriff des "Terminkontraktmarktes" wird von Streit (1979a) benutzt. Streit unterscheidet hiermit erstmals in der deutschen Literatur zwischen "Forward Market" und "Futures Market", die ublicherweise immer mit dem Begriff des Termınmarktes belegt wurden. 
Als eine zwischen Nach frager und Anbieter geschaltete Institution ubernimmt die Börse nicht nur die Marktgegenseite als Handelsvermittler, sondern sie garantiert auch die Erfüllung der gehandelten Kontrakte.

Der größte Teıl des Terminkontrakthandels erfährt durch dıe Vornahme entsprechender Gegengeschäfte vor Fälligkeit eıne Annullierung der Liefer-bzw. Abnahmeverpflichtung eines Gutes. Es kommt in diesen Fällen lediglich zu einem Zahlungsverkehr mit der Börse. Während Termingeschäfte auf eine Lieferung ausgerichtet sind, werden Terminkontrakte als temporäre Substitute für beabsichtigte Kassamarkttransaktionen benutzt. Streit1) kennzeichnet deshalb diese Art des Handels als ein "kontraktbezogenes Direktgeschäft."

Terminkontraktmärkte existieren für eine Vielzahl von Gütern mit unterschiedlicher zeitlicher Tiefe an verschiedenen Orten. Die bedeutendsten Handelsplätze sind2)

- die London Metal Exchange (für Aluminium, Blei, Kupfer, Silber, Zink, Zinn),

- der Chicago Board of Trade (für Silber) und

- die Commodity Exchange, Incorporation, New York (für Gold, Kupfer, Silber, Zink).

1) Streit (1979b), S.6. Zu den Funktionen und Funktionsvoraussetzungen bzw. -hemmnissen siehe Streit (1979a), S.17-34.

2) Siehe hierzu Keyser (1979), S.212, 224 und 247. Für Agrarprodukte existieren ebenfalls verschiedene Handelsplätze. 
Optionsmarkt (Option-Market): 1)

Auf dem Termin- und dem Termınkontraktmarkt beınhalten die Vertráge Rechte und Pflichten bezüglich der zukunftigen Lieferung eines Gutes. Einerseits hat der Käufer das Recht auf Lieferung und die Pflicht zur Abnahme, andererseits steht dem Verkäufer das Recht auf Abnahme zu und die Pflicht zur Lieferung.

Ein Optionskontrakt dagegen beinhaltet nur Rechte auf zukünftıg handelbare Güter. Der Käufer eınes Optionskontraktes erhält entweder das Recht auf die Lieferung (Kaufoption) oder das Recht auf die Abnahme (Verkaufoption) einer bestimmten Quantität und Qualıtät eines Gutes zu einem bestimmten Preis in einem bestimmten Zeitraum. Je nach der tatsächlichen Preisentwicklung wird der Optionskäufer dann von seinem Recht Gebrauch machen oder es verfallen lassen.

Demnach wäre der Optionshandel als ein eingeschränktes güterbezogenes Vorausgeschäft zu kennzeichnen. Die Einschränkung bezieht sich dabei auf die Wahlmöglichkeit des Optionskäufers, sein Vertragsrecht zu nutzen oder nicht.

Optionskontrakte wurden schon um 1920 gezeichnet.2) Optionsmärkte für Rohstoffe findet man in London (für alle Metalle) und in Sidney (für Gold).3)

1) Ausführungen zu Optıonsmärkten findet man bspw. bei Burns (1979), S.57-67 und bes Butler (1979).

2) Burns (1979), 557.

3) Butler (1979), S.35. Auch für Agrarprodukte und Wertpapıere existıeren Optionsmarkte (Chicago Board Options Exchange). 
Langfristige Lieferverträge:

Der Handel auf dem Terminmarkt war als ein güterbezogenes Vorausgeschäft gekennzeichnet worden. Zwei Handelspartner vereinbaren für einen bestimmten Zeitpunkt die einmalige Lieferung bzw. Abnahme einer bestimmten Gütermenge mit bestimmten Eigenschaften. Würden Terminmärkte für jeden Zeitpunkt existieren, dann lägen ähnliche Abschlüsse vor, wie sie in langfristigen Lieferverträgen zum Ausdruck kommen. Zur Abgrenzung der Abschlüsse in langfristigen Lieferverträgen gegenüber Terminmärkten können drei Merkmale genannt werden:

(1) Mehrmaliger Güteraustausch innerhalb eines langfristigen Liefervertrages, einmaliger Güteraustausch im Terminhandel;

(2) größerer inhaltlicher Gestaltungsspielraum in langfristigen Lieferverträgen;

(3) langfristige Lieferverträge sind das Ergebnis eines (bilateralen) Verhandlungprozesses, Termingeschäfte sind das Ergebnis eines Marktprozesses. 1)

Gemäß dieser Abgrenzung kann ein langfristiger Liefervertrag bezeichnet werden als eine Menge güterbezogener Vorausgeschäfte. Der langfristige Liefervertrag ist anzusehen als ein Ersatz2) für das Fehlen der Möglichkeit, Abschlüsse auf den Terminmärkten in individuell wünschbarer Weise zusammenzufügen.

Das Marktrisiko für einen Abbauunternehmer besteht darin,

- ob eine Nachfrage für seinen Rohstoff zukünftig existiert,

- welchen zeitlichen Verlauf die Nachfrage hat

- und von welcher Dauer sie ist.

1) Vgl. Dasgupta/Heal (1979), S.109.

2) Auf diese Eigenschaften der langfristigen Verträge, (partielle) Substitute für fehlende Zukunftsmärkte zu sein, weisen auch hin Nordhaus (1973), S.534; Dasgupta/Heal (1979), S.109, Siebert (1983), S.264. 
Für den Abbauunternehmer stellt sich die Frage, ob er seine Fördermengen uber langfristige Lieferverträge vorausverkaufen und damit sichere Gewinnchancen nutzen soll, oder soll er seine Fördermengen für Kassaverkäufe bereithalten, um unerwartete Preissteigerungen nutzen zu können? Letzterem stehen aber auch Verlustmöglichkeiten gegenüber. Diese könnten begrenzt werden, wenn der Abbauunternehmer geeignete Transaktionen auf einem Terminkontraktmarkt vornehmen würde. 1) Ob ein Abbauunternehmer langfristige Lieferverträge oder Terminkontrakte als Instrument zur Absicherung gegen Preisrisiken wählt, hängt ab von der Einschätzung der Nutzen und Kosten, die diese verursachen. Zur Vermeidung von Preisrisiken sind langfristige Lieferverträge geeigneter als Terminkontrakte.2) Nur wenn sich Kassa- und Terminkontraktpreise parallel entwickeln, kann das Preisrisiko über Terminkontrakttransaktionen vermieden werden.

Gegenüber langfristigen Lieferverträgen haben Terminkontrakte den Vorteil, daß ihre Erfüllung von der "clearing-Stelle" der Börse garantiert wird. Das Risiko der Zahlungsunfähigkeit des Handelspartners im Falle der langfristigen Lieferverträge existiert bei Terminkontrakten nicht.

Aufgrund der verminderten Anzahl an Transaktionen bei langfristigen Lieferverträgen und aufgrund der Standardisierung und der Existenz einer Börsenorganisation bei Terminkontrakten werden die Transaktionskosten3) für diese Handelsarten geringer sein als bei Termingeschäften.

Langfristige Lieferverträge ermöglichen aufgrund festgeschriebener Allokationsmuster eine stabile (kooperative) Beziehung zwischen den Handelspartnern trotz Marktrisiken. Insofern schaffen langfristige Lieferverträge eine "Quasi-Integration" zwischen Abbauunternehmen und Verarbeitungsunternehmen.4) Verglichen mit vertikal integrierten Unterneh-

1) Um diesen Vorteil von Terminkontrakten gegenüber Lieferverträgen abzuleiten, wird von einigen Autoren das Konzept des "convenience yield" benutzt. Vgl. Telser (1981), S.7.

2) Vgl. Telser(1981), S.5.

3) Kosten der Suche nach geeigneten Handelspartnern, Kosten der Qualitätsprüfung, Beurkundungskosten, Kommunikationskosten, etc.

4) Vgl. Smith, B. $(1978,1979)$. 
men 1) ist eine Unternehmensbeziehung aufgrund langfristıger Lieferverträge auf Datenänderungen reagibler. Neuen Verarbeitungsunternehmen ist der Zugang zu Rohstoffquellen bei einer bestehenden Vertragsstruktur leichter möglich als bei vertikaler Integration. Neuen Abbauunternehmen mit günstigeren Abbaukosten oder neuen Verarbeitungsunternehmen mit effizienteren Verarbeitungstechniken wird es zwar schwerfallen, in einer bestehenden Vertragsstruktur geeıgnete Handelspartner zu finden, jedoch brauchen die Vereinbarungen nur hinreichend flexibel gemacht zu werden, 2) um auch hier Anpassungsprozesse nicht auszuschließen.

Besondere Bedeutung kommt langfristigen Lieferverträgen dort zu, wo ohne die Festschreibung eines Allokationsmusters ein Ressourcenabbau nicht zustande käme. Dies ist bei Erdgas gänzlich und bei Kohle und Erdöl teilweise der Fall.3)

Zu den Ursachen der Existenz von Terminkontraktmärkten führt Telser4) aus, daß nicht der Bedarf an Preissicherheit als ursächlich für die Existenz der Termınkontraktmärkte angesehen werden könne, sondern der Bedarf an vertretbaren Finanzaktiva.5) Die geringe Anzahl der Kontraktlaufzeiten spräche vielmehr für eine liquiditätstheoretische Begründung der Existenz von Terminkontraktmärkten.

Terminkontraktmärkten mag zwar nur eine geringe Bedeutung bei der Vermeidung von Preisrisiken (über Hedging) zukommen, bei der Informationsbeschaffung, -verarbeitung und -weitergabe jedoch kommt den Ter-

1) Gordon (1983), S.414 bezeichnet vertikale Integration und langfristige Lieferverträge als die zwei "polaren" Institutionen zur Reduktion von Marktrisiko, zwischen denen alle anderen Institutionen anzusiedeln sind.

2) Siehe hierzu den nächsten Abschnitt.

3) Zur besonderen Problematik langfristiger Verträge für Erdgas siehe Schneider/Schulz (1983), für Kohle siehe Gordon (1983) und für Erdöl Barrows (1980).

4) Telser (1981), S.7f.

5) Houthakker (1959), S.146f und Working (1970), S 29 vergleichen ebenfalls die Rolle von Termınkontrakten mit der von Wahrungseinheiten. 
minkontraktmärkten große Bedeutung zu.1) Bspw. werden die Börsenpreise der London Metal Exchange für Kupfer als Grundlage für Vereinbarungen über Kupferlieferungen gemacht.2) Peck (1976) zeigt, daß Preisausschläge auf den Kassamärkten gedämpft werden, bzw. gegen einen langfristigen Trend konvergieren, wenn Terminkontraktpreise Grundlage für die Produktionsentscheidungen sind. Über diesen "Umweg" eines verbesserten Informationsstandes durch funktionierende Terminkontraktmärkte sinkt für die Unternehmen das Preisrisiko.

Der nächste Abschnitt befaßt sich nun eingehender mit langfristigen Lieferverträgen, denn diesen kommt im Rohstoffhandel zwischen Abbau- und Verarbeitungsunternehmen empirisch die größte Bedeutung zu.

\subsubsection{Langfristige Lieferverträge auf dem Rohstoffmarkt}

Rohstoffverarbeitende Unternehmen sind an einer sicheren Rohstoffversorgung zum Zwecke einer kontinuierlichen Produktionstätigkeit interessiert. Durch Verwendungstechnologien können strenge Restriktionen für den Einsatz bestimmter Rohstoffe bestehen. Beispielsweise sind Eisenerz oder Kohle kein homogenes Gut, sondern erfordern für den weiteren Einsatz je nach Qualität verschiedene Technologien. Hat ein Verarbeiter eine bestimmte Technologie gewählt, so kann er auch langfristig nur schwer substituieren. Ausfälle bei der Rohstoffversorgung würden spätestens nach einem eventuellen Lagerabbau zu Produktionsausfällen führen.

Abbauunternehmen wünschen nicht nur aus Gründen einer kontinuierlichen Abbautätigkeit eine sichere Absatzbasis und einen sicheren Zugang

1) Hierzu Streit/Quick (1981).

2) Vgl.Radetzkı (1978), S.123. 
zu Rohstofflagerstätten. Lange Vorlaufzeiten und hohe Betràge für Investitionen in die Erforschung und Entwicklung von Lagerstätten machen uber längere Frist die Kenntnis von Abnahmemengen erforderlich.

Für den Abschluß langfristiger Verträge werden als Anreize in der Literatur genannt:1)

- Verteilung von Risiken

- Übertragung von Risiken

- Beseitigung des "moral hazard"

- Ausnutzung unterschiedlicher Erwartungen

- Koordination komplementarer Produktionsfaktoren

- Ausdehnung der Monopolmacht

- Verringerung der Transaktionskosten.

Bis auf geringe Ausnahmen erfolgt der Handel mit Rohstoffen, die nicht Brennstoffe sind, über langfristige Lieferverträge.2) Beispielsweise wurden Ende der sechzıger Jahre fast $40 \%$ der Welteisenproduktion über langfristige Lieferverträge und nahezu $20 \%$ über kurzfristige Lieferverträge (ein Jahr) gehandelt. 3) Japan als einer der Hauptimporteure für Eisen hat 1968 $96 \%$ seines Eisenbedarfs über langfristige vertragliche Lieferungen gedeckt. In den USA dagegen wurde für den gleichen Zeitraum der Eisenbedarf der Stahlgesellschaften zu $96 \%$ aus Minen gedeckt, die ihnen über vertikale Integration angeschlossen waren.

Der größte Teil des Rohöls wird ebenfalls auf der Basis langfristiger Verträge zwischen Förderländern und Raffinerien gehandelt. Was für Erdöl gilt, trifft auch für die Primärenergieträger Kohle und Erdgas zu.

Am Beispiel Erdöl zeigt Schaubild 4.1 die Verteilung der Importmengen der IEA-Länder auf den Handel über langfristige Verträge (Konzessions-, Rückkaufs-, Regierungsöl) bzw. den Kassamarkt.

1) Diese werden zusammenfassend genannt bei Roberts (1980), S.246.

2) Vgl. Banks (1980), 5478 .

3) BossonNaron (1977), S.108. 
Schaubild 4.1: Roholimportstruktur der IEA-Lander im Jahre 1980 in $\mathrm{mbd} / \%$

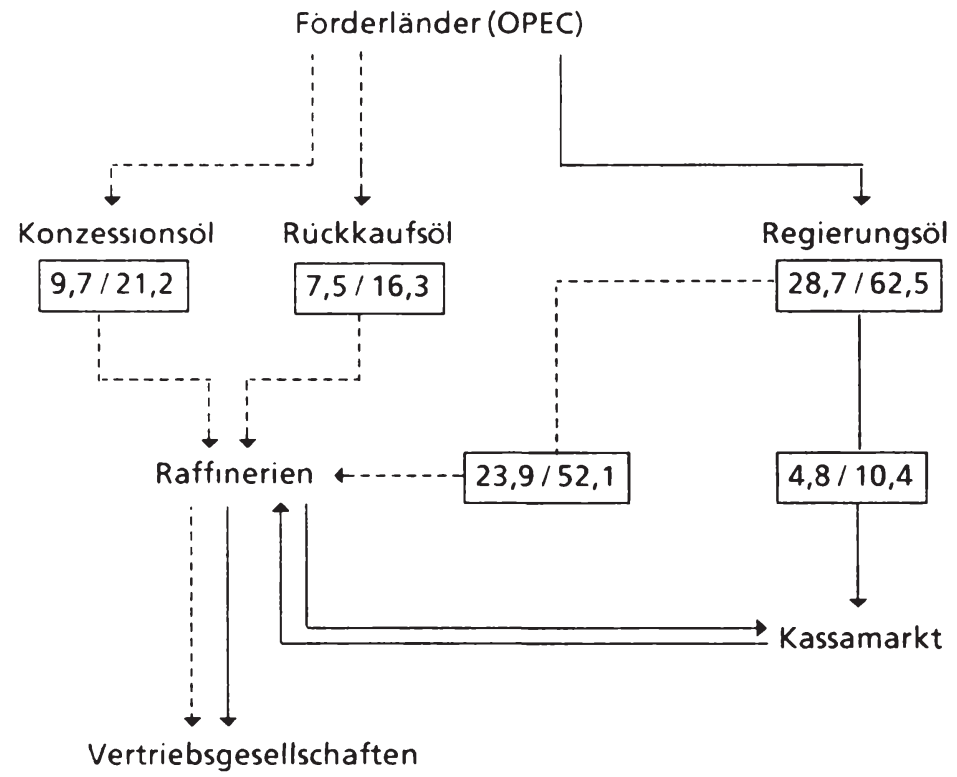

Quelle: Zusammengestellt nach Angaben in Mohnfeld (1982), S.185 u. 187.

Bisher war argumentiert worden, daß Unsicherheit über Nachfrage bzw. Angebot auf zukünftigen Kassamärkten einen Bedarf an langfristigen Verträgen begrundet. Bei Burchard 1) findet man die Umkehrung dieser Folgerung: Langfristıge Lieferverträge schaffen einen Bedarf an kurzfristigen Ausgleichsmarkten.

1) Burchard (1980), S.470. 
Für die Existenz eines kurzfristigen Marktes neben dem langfristigen Vertragshandel, kann es je nach Rohstoff unterschiedliche Grunde geben: 1)

- Produktions- und Absatzstruktur fallen aufgrund der Kuppelproduktionstechnik auseinander.

- Die Nachfragen nach Rohstoffprodukten haben unterschiedliche saisonale Schwankungen. Eine Kuppelproduktion verhindert hier eine Anpassung.

- Die Nachfrage nach Rohstoffprodukten ist unsicher.

Auf die Allokationswirkungen einer Kuppelproduktions-bzw Kuppelabbautechnologie soll hier nicht weiter eingegangen werden.2) Zu erörtern bleibt, welcher Art die inhaltlichen Ausgestaltungen langfristiger Lieferverträge sind.

Die Vertragsinhalte lassen sich für einen bestimmten Rohstoff eintellen 3 ) in Vereinbarungen über die Mengen, die Preise, die Vertragsdauer, den Transport und die Handhabung "bedeutender Situationsveränderungen."

\section{Mengen:}

An Mengenvereinbarungen ist je nach Wunsch der Handelspartner ein breites Spektrum möglich.

- Die schwächste Form ist eine Absichtserklärung, in der sich beide Vertragspartner zum Handel einer bestimmten Menge bereit erklären, aber nicht dazu verpflichten.

- Eine Verpflichtungserklärung innerhalb bestimmter Grenzen wäre eine stärkere Form der Vereinbarung, d.h. Käufer und Verkäufer verpflichten sich, bestimmte Mindestmengen abzunehmen bzw. anzubieten.

1) Vgl. Burchard (1980), S.470.

2) Hierzu siehe Pindyck (1981).

3) Nach Smith, B. (1979), S.69ff. 
- Weiter kann das Handelsvolumen in Antellswerten festgelegt werden, so daß das absolute Volumen wieder schwanken kann. Die Vertragspartner verpflichten sich, einen bestimmten Anteil ihrer Nachfrage bzw. ihres Angebotes auf den jeweıligen Partner entfallen zu lassen.

- Die starkste Form der Festlegung ist die Verpflichtung zur Abnahme bzw. zur Lieferung fester Mengeneınheiten pro Zeitpunkt oder -raum.

Welcher Form die Handelspartner den Vorzug geben, entscheiden sie durch Abwàgen der für sıe individuell daraus anfallenden Vor- und Nachteile.

Beispielsweise wird ein Abbauunternehmer, der sich einer stabilen Nachfrage gegenubersieht, aber selbst Produktionsschwankungen unterliegt, an einem Vertrag mit "variabler" Mengenfestlegung interessiert sein.

Mit den Mengenfestlegungen werden meist auch gleichzeitig Vereinbarungen über das Ausmaß von Kompensationszahlungen getroffen für den Fall, daß Mengenverpflichtungen und Qualitätszusagen nicht eingehalten werden.

Preise:

Der Begriff der Versorgungs- oder Absatzsicherheit beinhaltet nicht nur sicheres Wissen um die Lieferung oder die Abnahme bestimmter Mengen, sondern auch sicheres Wissen darüber, daß diese Mengen zu bestimmten Preisen erhalten werden.

Nominale Festsetzungen in einer bestimmten Währung sind auflange Frist ungeeignet. Ublıch sind Preisgleitklauseln mit verschiedenen Indexierungsarten. Man versucht damit, Veränderungen der Währungsparitäten oder bestimmter Preısniveaus auf adäquate Weise für beide Vertragspartner zu berücksichtigen. Denkbar sind auch Vereinbarungen, die die Preise hinreichend flexibel lassen, um auf kostengünstigere Abbaualternatıven reagıeren zu können. 
Vertragsdauer:

Für langfrıstige Lieferverträge hat sich im Bergbau eine Laufzeit von 10 bis 12 Jahren durchgesetzt. 1) Bei Erdgas liegen die Laufzeiten zwischen 15 und 25 Jahren.2) Diese Laufzeiten scheinen bestimmt zu sein von dem schon angesprochenen Rentabilitätsaspekt, von der Möglichkeit, solche Verträge beleihen zu können, von der Anzahl alternativer Vertragspartner und von technologischen Faktoren. Besonders die Einschränkung der Alternativen aufgrund technologischer Restriktionen spricht für längerfristige Vertragszeiten von fünfund zwanzig und mehr Jahren. 3 )

Transport und Situationsveränderungen:

Die Durchführung des Transports wird gewöhnlich gänzlich von einem der beiden Handelspartner übernommen. Damit liegt aber noch nicht fest, daß dieser auch die Kosten trägt. In den meisten Fällen werden jedoch die Kosten von dem Käufer übernommen. Daneben findet man Vereinbarungen darüber, wie sich bedeutende Situationsveränderungen auf die weitere Entwicklung des Vertragsverhältnisses auswirken sollen. Diese Vereinbarungen schließen auch die Bestimmung von Schlichtungsverfahren für den Streitfall ein.

1) Smith, B. (1979), S.69.

2) Schneider/Schulz (1983), S.382.

3) Smith, B. (1979), S.70. 


\subsection{Substitutive Allokationsmechanismen für Reserven}

\subsubsection{Auktion, diskretionäre Vergabe, Verhandlung}

Einen Reservenmarkt, der in ein vollständiges System von Zukunftsmärkten eingebettet ist, findet man realiter nicht vor. Was man vorfindet, sind verschiedene Formen von Allokationsmechanısmen, die (ersatzweise) die Verteilung der Reservenbestände über Nutzungsrechte vornehmen.

In den USA existiert ein Markt für Abbaurechte auf Erdgas. Für die Förderung von Erdöl im äußeren Kontinentalschelf werden vom Innenministerium Lizenzen über einen Auktionierungsmechanismus vergeben. 1)

Da außer in den USA ein Landbesitzer kein Eigentumsrecht an den unter seınem Land auffindbaren Bodenschätzen hat, findet man sonst nirgends einen Markt für Abbaurechte. Die förderbaren Ressourcen sind in allen anderen Ländern Staatseigentum. Will ein Unternehmen Ressourcen abbauen, so muß es von der Regierung das Recht dazu erwerben. Die Lizenzvergabe über Auktionierung findet man ebenfalls nur in den USA. Ansonsten werden Lizenzen über diskretionäre Mechanismen vergeben2) (bspw. in England und in Norwegen).

In den verschiedenen Entwicklungsländern3) werden meist unter der Organisationsform eines Gemeinschaftsunternehmens4) (joint venture) Ressourcen gefördert. Die fiskalischen Ausgestaltungen der einzelnen, das Gemeınschaftsunternehmen begründenden Vereinbarung können dabei sehr unterschiedlich sein. Sie sind Ergebnis eines Verhandlungsprozesses.

1) Gilley/Karels (1981), Smith, J. L. (1980, 1982).

2) $\operatorname{Dam}(1976)$.

3) Schanze, u.a. (1981).

4) Zum Begriff des Gemeınschaftsunternehmens Doo-Soon Ahn (1981), S.51-58. 
An Allokationsmechanismen für Reserven findet man also vor: den Markt, die Auktion, eıne diskretıonàre Vergabe und die Verhandlung.

Preise, Gebote (über Förderabgabe, Bonus, etc.), admınıstrativ oder politisch entstandene Zielvorstellungen (Nationalıtät des Forderunternehmens, Fördergeschwındigkeit, etc.), Strategien und/oder Macht bilden in diesen Mechanismen die Kriterien, nach denen Reserven auf Abbauunternehmen verteilt werden.

Da der Markt als Allokationsmechanismus in Abschnitt 6.2. diskutiert wird, soll hier nicht weiter auf ihn eingegangen werden.

Mit einer Auktion versucht der Staat eine Rente 1) zu erhalten, die ihm 2) als Entgelt für die abgebauten Rohstoffeinheiten dienen soll. Dieselbe Absicht haben auch die Beamten, die diskretionär Lizenzen vergeben, bzw. die Unterhändler, die auf dem Verhandlungswege Abbaurechte vertellen.

Im Unterschied zur Auktion fehlt bei den beiden letzten Mechanismen die Konkurrenzsituation des Gegeneinanderbietens. Der Lizenzgeber (Reservenbesitzer, Regierung) möchte uber die Konkurrenzsituation der Auktion -der Höchstbietende erhält den Zuschlag - die ökonomische Rente der Ressource abschöpfen. Der Lizenznehmer (Förderunternehmen) ist bereıt, über sein Gebot einen Teil der ökonomischen Rente abzugeben.

Aufgrund des Risikos, das mit dem Bıeten verbunden ist, wird das Förderunternehmen jedoch nicht die gesamte erwartete ökonomische Rente abgeben.3) Mit der Höhe des Gebotes steigt zwar die Wahrscheinlichkeit, den

1) Hierzu bspw. Reece (1979).

2) Genauer, den Individuen dieser Volkswirtschaft.

3) Vgl. Reece (1978), S.383 bzw. Smith, J. L. (1980), S. 19 in einem einfacheren Modell. Reece $(1978,1979)$ muß in seinen allgemeineren Ansätzen auf Computerlösungen numerischer Techniken zurückgreifen, da seine Ansätze analytisch schwer handhabbar sınd. Bei Wilson (1977), S.517 findet man die Aussage: Gehe die Zahl der bietenden Konkurrenten gegen unendlich, dann konvergiere der Erwartungswert des gewinnenden Gebotes gegen den wahren Wert der Lizenz. Diese Konvergenzaussage wird jedoch nıcht aus einem Optımierungsproblem abgeleitet, sondern aus einem Bayes-Ansatz. 
Zuschlag zu erhalten, andererseits jedoch mindert das Gebot den Nettowert der Lizenz, der sich als Differenz zwischen dem erwarteten Wert des Ressourcenbestandes und dem zu zahlenden Gebot ermittelt.

Der Wert für den zu bietenden Reservenbestand ist mıt eıner Vielzahl von Risiken behaftet. Geophysikalische Prognosen über die Größe und die Qualität eines Bestandes liefern dabeı den großten Beıtrag zum Gesamtrısiko.1) Weitere Risikoursachen findet man in den Prognosen úber zukünftige Preise und Abbaukosten. Unterschiedliche Prognosen über diese Größen führen zu unterschiedlichen Geboten auf dem Auktionsmarkt und haben welter auch unterschiedliche Abbauprofile zur Folge.

Auktionen werden in der Literatur unter zwei Aspekten betrachtet.2) Einerseits aus der Sicht des Lizenzgebers (bspw. Reece (1979)) und andererseits aus der Sicht des Lizenznehmers (bspw. Smith $(1980,1982)$ ).

Reece (1979) betrachtet verschiedene Variable (Bonus, Gewinnanteil, Royalty) als Gegenstand des Gebotes und analysiert deren Wirkungen auf die Verteilung der okonomischen Rente zwischen Lizenzgeber und -nehmer. Will der Lizenzgeber einen móglichst großen Teil der Rente abschöpfen, dann ist als Gegenstand des Gebotes der Gewınnanteil zu wählen. Von der ökonomischen Rente schöpft der Lizenzgeber am wenigsten ab, wenn er über einen Bonus bieten läßt. 3)

Smith (1982) diskutiert in einer empirischen Studie den Einfluß der Firmengröße, der Anzahl der mitbietenden Konkurrenten und der Gewinnerwartungen auf die Höhe des Gebotes.

1) Vgl. hierzu Smiley (1979), S.9f

2) Zu einer detaillierten Übersicht über Auktionsmodelle siehe Smith, J. L. (1980), S.9-28 und die dort angegebene Literatur.

3) Reece (1979), S.664ff. 
Ist Reece an Aussagen über die Verteilung der Rente in Abhängıgkeit von der zu bietenden Variablen interessiert und Smith an Aussagen über die Bestimmungsfaktoren eines Gebotes, so findet man bei Leland (1978) Aussagen über die Verteilung des Risikos zwischen Lizenzgeber und Lizenznehmer in Abhängigkeit vom Risikoverhalten.1) Ein Bonus-Bieten ist nıcht geeignet zur Risikoverteilung. Der Lizenznehmer trägt das gesamte anfallende Risiko. Zur Risikoverteilung geeignet sind Auktionen, die als Gegenstand des Bietens den Gewinnantell oder eine Royalty haben. Sind Lizenzgeber und Lizenznehmer risıkoavers, dann ist eine gewinnabhängige Entlohnung der Lizenz für beide optimal im Sinne einer individuellen Erwartungsnutzenmaximierung.

Mit diesen Aussagen Lelands kann das Ergebnis Reeces zur Rangfolge der Vorteilhaftigkeit der verschiedenen Auktionen für den Lizenzgeber begründet werden. Je größer das aus der Sicht des Lizenznehmers an den Lizenzgeber übertragene Risiko ist, desto größer ist auch die Zahlungsbereitschaft des Lizenznehmers (bzw. die Zahlungsforderung des Lizenzgebers). Im Falle einer Bonus-Auktion fällt die Lizenzgebühr am kleinsten aus, da der Lizenznehmer das gesamte Risiko trägt. Denn unabhängig vom später realisierten Wert des Ressourcenbestandes ist vorab ein Bonus zu zahlen. Mit der Gewinnanteil-oder der Royalty-Auktion hingegen übernimmt der Lizenzgeber einen Teil des Risikos, da seine Entlohnung mit dem Gewınn oder der Royalty variiert.

Die Höhe des Bonus-Gebotes hat keinen Einfluß auf die intertemporale Allokation des Ressourcenbestandes, da der Bonus als eine fixe, vor dem $A b$ bau anfallende Kostengröße in das intertemporale Marginalkalkül zur Be-

1) Leland (1978), S.418f. 
stimmung des Abbauprogrammes nicht eingeht. Denn für die Abbauentscheidung eines Unternehmers sind die Ergebnisse der Auktion relevant, die er in seınem intertemporalen Optimierungskalkúl in den eınzelnen Perioden zu berucksichtigen hat.

Auf das Abbauprofil haben keinen Einfluß die Unterscheidungen 1)

- offene oder geheime Auktion2)

- einzelnes oder gemeinsames Bieten

- mit oder ohne Bonus-Gebot bzw. Gebot für eine flächenbezogene Förderabgabe.

Einfluß auf die zeitliche Entwicklung der Abbaumengen haben die Tatbestände, ob

- eine diskriminierende oder eine nichtdiskriminierende Auktion bezüglich einer abbaumengenbezogenen Förderabgabe vorliegt,

- eine progressiv von den Abbaumengen abhängıge Förderabgabe Gegenstand des Gebotes ist oder nicht.

Im Falle einer diskriminierenden Auktion hat der Höchstbietende auch die von ihm gebotene Förderabgabe zu entrichten. Eine nichtdiskriminierende Auktion fordert von dem Höchstbietenden nur die Zahlung des zweithöchsten Gebotes. Betrachtet man sich den Abbauunternehmer des Abschnitts 2 und interpretiert das Gebot als Gegenwartswert des Preises für ein Abbaurecht, so wird der Abbauunternehmer im Falle einer nichtdiskriminierenden Auktion aufgrund eines geringeren Preises für die Reserve den Reservenbestand schneller abbauen.

Für eine progressive Förderabgabe kann der gegenteilige Allokationseffekt festgestellt werden. Der Abbauunternehmer wird mit einer geringeren Ab-

1) Auf die Höhe des Gebotes und damit die Entscheidung, ob abgebaut wird oder nicht, haben diese Elemente einen Einfluß.

2) Zu den verschiedenen Arten der Auktionierung vgl Holt (1980), S.434. 
baumenge zu Anfang fördern und über einen längeren Zeitraum mit geringeren Abnahmeraten der Fördermengen die Reserven abbauen.

Die eben gemachten Aussagen hinsichtlich der Bedeutung von Ergebnissen unterschiedlicher Auktionsarten für die intertemporale Allokation gelten auch für die Auswirkungen der Ergebnisse eines Verhandlungsprozesses bzw. für die Anforderungen bei diskretionärer Vergabe.

Für die intertemporale Allokation ist es letztendlich unbedeutend, durch welchen Mechanismus ein bestimmter Preispfad für die Reserven zustandekommt. Relevant ist die Tatsache, ob durch unterschiedliche Mechanismen auch unterschiedliche Preispfade zustandekommen. Jeder der genannten Mechanismen kann die Vereinbarungen entstehen lassen, wie sie in Abschnitt 6.2. auf ihre Allokationswirkungen analysiert werden. Bedeutend sind dann Unterschiede bei der Preisbildung zwischen den einzelnen Vereinbarungen.

Der nächste Abschnitt stellt mögliche, real existierende Vereinbarungsarten dar.

\subsubsection{Vertragsvereinbarungen auf dem Reservenmarkt}

Bei neueren Projekten der Erschließung von Rohstofflagerstätten im Metallerzbergbau in Entwicklungsländern1) findet man überwiegend die Organisationsform der Gemeinschaftsunternehmung zwischen dem rohstoffbesitzenden Staat und einem investitionsbereiten Abbauunternehmen. Das

1) Vgl. hierzu Schanze (1981). 
Gemeınschaftsunternehmen 1) ist nicht im Sinne eıner spezifischen Vertragsvereınbarung zu verstehen wie sie hier betrachtet werden soll, sondern als eıne projektbezogene Partnerschaft. Beim Erdöl zeichnet sich im Gegensatz dazu eine Entwicklung zugunsten von Dienstleistungsverträgen zwischen Staatsgesellschaften und Mineralölgesellschaften ab, die früher als Konzessionäre Erdöl gefördert haben:2)

Seit 1960 existieren neben den Konzessionen Abbaumengenteilungsvertráge. 1970 kamen Verträge zur Risikoteilung hinzu. Mitte der siebziger Jahre entwickelten sich daraus umfassende Dienstleistungsverträge. Hinsichtlich der Übernahme der Finanzierung eines Projektes, der Aufteilung des Risikos3) und der Verteilung der Erträge unterscheiden sich diese Verträge voneinander.

Bestehen in älteren Vereinbarungen zu Rohstoffprojekten die Erträge des Rohstofflandes in Förderabgaben, Ertrag-, Umsatz-, Ausfuhrsteuern, Pachtzinsen und/oder verschiedenen Bonı, so verlagert sich die Ertragserzielung in neueren Vereinbarungen auf die Gewinnteilung durch Kapitalbeteiligung. Dies kann bis hin zur Verstaatlichung des Projektunternehmens gehen. Das investierende Abbauunternehmen arbeitet dann als Dienstleistungsunternehmen gegen Honorar und Kostenerstattung.

Im folgenden sollen nacheinander kurz erörtert werden

(1) die Konzession (Concession, Permit, Lease, License),

(2) die Abbaumengenteilung (Production-sharing-contract),

(3) die Risikoübernahme (Risk-service-contract) und

(4) der Dienstleistungsvertrag (Service-contract).

1) Zu den wesentlichen Merkmalen eines Gemeınschaftsunternehmens siehe Doo-Soon Ahn (1981), S.58.

2) SmithMells (1975), Fritzsche (1979), Barrows (1980).

3) Risiko hinsichtlich Erkundung, Erforschung, Entwicklung eines Lagers, der Produktion und der Nachfrage. 
Als Unterscheidungsmerkmale werden die Verfügbarkeit über die Abbaumengen und die Erhebung einer Förderabgabe bzw. deren Ausgestaltung verwendet. Die Modellbildung des Abschnitts 6.2. greıft diese Kriterien auf.

Mit einer Konzession erhält das Abbauunternehmen das Recht, nach Reserven zu suchen und im Erfolgsfall diese auch abzubauen. Das Abbauunternehmen trägt alle anfallenden Kosten und zahlt pro abgebauter Rohstoffeinheit eine Abgabe an den Konzessionsgeber. 1)

In über 120 Ländern werden Konzessionen zur Erdölförderung vergeben.2) Die Förderabgabe beläuft sich je nach Land auf 12 bis $40 \%$ eines bestimmten Listenpreises für Rohöl.3)

Eine" production-sharing"-Vereinbarung teilt die Abbaumengen zwischen dem Reservenbesitzer und dem Förderunternehmen. Das Förderunternehmen trägt auch hier alle anfallenden Kosten. Die relative Abgabenbelastung für das Abbauunternehmen hinsichtlich Förderabgaben, Einkommensteuer, etc. ist geringer als bei Konzessionen.

Eine Teilung der Abbaumengen hat für das Rohstoffland und das Abbauunternehmen den Vorteil, daß das Marktrisiko geteilt wird. Für das Rohstoffland kann es im Gegensatz zur Konzession vorteilhaft sein, über Rohstoffmengen selbst zu verfügen und diese in eigener Verantwortung zu vermarkten. Einige Rohstoffländer treten einen Teil der Abbaumengen an die Förderunternehmen zur Vergütung von deren Kosten ab. Erst die restliche Abbaumenge wird dann auf beide aufgeteilt.

1) Bspw. zahlte die Gesellschaft "Getty Oil" an Saudi Arabien eine Abgabe von 55 cents je barrel Rohöl [so Smith/Wells (1975), S.32].

2) Vgl. Barrows (1980), S.44.

3) England und Norwegen besitzen daneben noch eine spezielle vom Rohstoffpreis abhängige Einkommensteuer. Zwischen 1979 und 1980 stieg der Steuersatz auf die Nettoeinnahmen aus Roholverkaufen von $45 \%$ auf $70 \%$ [nach Barrows (1980), S.45]. 
In Indonesien werden den Förderunternehmen 40\% der Abbaumengen zur Kostendeckung abgetreten. Der Rest teilt sich dann 35 zu $65 \%$ zugunsten des Staates. In anderen Ländern liegt der Anteil für die Kostenvergütung zwischen 20 und $40 \%$. Für die restliche Abbaumengenteilung findet man Verteilungsquoten zwischen $85 \mathrm{zu} 15 \%$ zugunsten des Staates und $15 \mathrm{zu}$ $85 \%$ zugunsten des Förderunternehmens. Die Verteilung kann auch mit dem Förderniveau schwanken.

In Abhängigkeit von der Verteilung der Fördermenge variiert die Einkommensteuer. Ist zum Beispiel in Libyen bei einer Abbaumengenteilung von $81 \mathrm{zu} 19 \%$ zugunsten der staatlichen Ölgesellschaft das Einkommen steuerfrei, so verlangt Indonesien bei einer Aufteilung von 65 zu $35 \%$ zu seinen Gunsten eine Einkommensteuer in Höhe von $56 \% .11$

Der "risk-service"-Vertrag überträgt dem dienstleistenden Unternehmen das Explorationsrisiko. Gegen ein Honorar stellt das Unternehmen Kapital für die Exploration und eventuell später für den Abbau zur Verfügung. Bleibt die Exploration ohne Erfolg, dann läuft der Vertrag aus. Kommt es zur Förderung, so wird das eingesetzte Kapital mit Zins und einer Risikogebühr zurückgezahlt. Die Vergütung erfolgt nicht in Rohstoffmengen. Das Unternehmen kann das Vorkaufrecht für die Rohstoffe erhalten.

In dieser Form steht der Risikoübernahmevertrag als Übergang zwischen dem Abbaumengenteilungsvertrag und einem Dienstleistungsvertrag. Denn einerseits trägt das explorierende Unternehmen ein Risiko wie im Falle der Abbaumengenteilung und erhält andererseits wie im Falle des Dienstleistungsvertrages keine Rohstoffmengen als Vergütung.

Der Risikoübernahmevertrag fällt aus der modelltheoretischen Betrachtung heraus, da ein Explorationsrisiko in dieser Arbeit nicht unterstellt und auf seine Allokationswirkungen hin analysiert wird. Der Reservenbesitzer

1) Zu den Zahlenangaben vgl. Barrows (1980), S.47f. 
baut nach erfolgreicher Exploration selbst ab oder vergıbt den Abbau über eınen Dienstleistungsvertrag.

Im Dienstleıstungsvertrag übernimmt ein Unternehmen die Fördertätigkeit, ohne das Risiko einer vorherıgen Explorationstätigkeit zu tragen. Für die Dienstleistung "Abbau und Vermarktung" erhält das Unternehmen e1ne Vergütung. 1)

Dienstleıstungsverträge haben sich als Ergebnis der zunehmenden Anzahl der Mehrheitsbeteiligungen der Rohstoffländer bei Rohstoffprojekten gebildet.2) Das Rohstoffland (Entwicklungsland) ist meist nicht fähig, das Projekt angemessen mit Technologie und Management auszustatten. Damit einem investierenden Unternehmen bei vorherrschender Machtposition des Rohstofflandes die Projektführung zukommt, wurden Dienstleistungsverträge konstruiert. Die vom Investor bereitgestellten Leistungen der Unternehmensführung und der technologischen Ausstattung werden voll vergütet.

Für ein Unternehmen, das Ressourcen sowohl abbaut als auch auf eigene Rechnung vermarktet, sind in diesem Zusammenhang neben seinen Erwartungen über zukünftige Marktpreise der Rohstoffe (u.a.) auch die aufgrund einer Vertragsvereinbarung anfallenden Abgaben relevant. Betrachtet werden sollen hier nur die Förderabgaben. Abgaben aufgrund einer Besteuerung3) oder sonstige Abgaben wie Pacht, Zölle, etc. sollen nicht in die Analyse einbezogen werden. Zwar sind die Förderabgaben in der Einkommensrechnung der Rohstoffländer mittlerweile relativ gering anzusetzen, jedoch kommt gerade innen die Lenkungsfunktion für die Verteilung der Ressourcenzu.

1) So erhielt zum Beispiel Aramco in Saudi Arabıen eine Verguttung von 15 cents je barrel [Barrows (1980), S.65].

2) Vgl. Fritzsche (1981), S.149ff.

3) Siehe hierzu bspw. Sinn (1983). 
Bis zur Einführung der Einkommensteuer waren die Förderabgaben die einzige Einnahmequelle der Rohstoffländer. Die Förderabgabe wurde als ein Entgelt für die abgebauten Rohstoffeinheiten eingeführt. Nur in wenigen Fällen wurde dabei die Abgabe mit der Förderung fällig.1) In den meisten Verträgen ist festgelegt, daß die Förderabgabe erst auf die abgesetzten Rohstoffmengen erhoben wird.

Nach dem starken Ansteigen der Rohstoffpreise wurde von der mengenbezogenen Erhebungsweise übergegangen zu einer wertbezogenen $\mathrm{Er}$ hebungsweise, die sich am Verkaufserlös orientiert. Einige Verträge beinhalten zwar eine mengenbezogene Abgabe, jedoch richtet sich diese aus an Listenpreisen für diesen Rohstoff.2)

Als weitere Bemessungsgrundlage findet man auch die Abbaukosten.3) Es soll damit eine von der Preissituation unabhängige Einkommensentwicklung ermöglicht werden.

Die relativen Förderabgaben über alle Rohstoffarten bewegen sich zwischen $1 \%$ und $40 \%$ des Verkaufserlöses. 4 ) In diesem Intervall findet man eine Vielzahl unterschiedlich gestaffelter Förderabgaben.5) Sie sind differenziert nach

- den Abbaumengen,6)

- der onshore-/offshore-Förderung, 7)

1) So im Vertrag zur Bauxitförderung in Jamaika.

2) So die Verträge für Kupfer, Nickel, Zinn und Bauxit in Indonesien.

3) So in Panama und Madagaskar. Abbaukosten als Bemessungsgrundlage führen zu demselben Allokationsergebnis wie eine mengenbezogene Förderabgabe (vgl. Abschnitt 6.2.1.1.).

4) In den Entwicklungsländern zwischen $1 \%$ und $25 \%$. Vgl. Fritzsche (1981), S.87.

5) Vgl. Barrows (1980), S.39.

6) In Sierra Leone für den Abbau von Titan oder ın den europäischen Ländern für Erdöl (Nıederlande, Norwegen).

7) In Nigeria bei Erdö. 
- der Wassertiefe und/oder der Entfernung vom Festlandsockel,1)

- den Qualıtäten, 2)

- dem Zeitablauf,3)

- der Rohstoffart, 4)

- dem Verarbeitungsstand.5)

Mit diesen Differenzierungen liefern die Reservenbesitzer den Abbauunternehmen Anreize, bestimmte Ressourcen nach Mengen, Lage, Qualität und Zeit bevorzugt abzubauen.

Geht man von der Intention der Förderabgabe als Faktorpreis für die Reservennutzung aus, so behindert die Differenzıerung der Förderabgabe die Funktion eines Faktorpreises als Knappheitsindikator.

Greift man auf das Beispiel der Differenzierung nach Qualitäten zurück, so findet man in Guyana für bessere Qualitäten von Erdöl höhere Förderabgaben. In einem Modellansatz von Hartwick (1978) werden Rohstoffläger mit minderer Qualität nach Rohstofflägern mit besserer Qualität abgebaut. Qualitätsunterschiede werden in Hartwicks Ansatz mit Unterschieden in den Abbaukosten modelliert. Führt man nun nach Qualitäten differenzierte Förderabgaben ein, dann kann sıch je nach Höhe der Förderabgabe die Abfolge der Nutzung von Reservenlägern verschiedener Qualitäten umkehren.

1) Für Erdöl in Thailand und in Guyana.

2) Guyana differenzıert sowohl nach Qualitat als auch nach Wassertiefe und Entfernung vom Festlandsockel.

3) So in Liberia bei der Forderung von Eisenerz; in Bolivien für Gold und Platin.

4) Zwischen Erdol und Erdgas in fast allen Ländern, die diese Primärenergieträger fördern.

5) In Indonesien sind die Forderabgaben für aufbereitetes Eısenerz geringer als fur nicht aufbereitetes. 
Auswirkungen solcher Differenzierungen können in einem Modellansatz betrachtet werden, wie er in Abschnitt 6.2.1. vorgestellt wird. Wie im Abschnitt 2.4. wird von einem zweistufigen Marktkonzept ausgegangen. Im Unterschied zu Abschnitt 2.4. wird dort dem Markt für Abbaurechte (für Ressourcen in situ) ein Rohstoffmarkt gegenübergestellt, für den nur zukünftige Kassamärkte, aber keine Terminmärkte existieren: Der Preis für das Abbaurecht (der Faktorpreis für die Reserve oder die Förderabgabe) ist eine determinıstische Variable, der Preis für den Rohstoff ist eine Zufallsvariable. 


\section{Die Allokationswirkungen institutionalisierter Marktsubsti- tute in Zweiperiodenmodellen des Ressourcenabbaus}

Die in Kapitel 4 erörterten Substitute für fehlende Zukunftsmärkte des Rohstoffhandels werden hier in Zweiperiodenmodellen diskutıert. Zweiperiodenmodelle haben die Vorteile der einfachen Handhabbarkeit und der Identität von closed- und open-loop Lösungen. Die genannten Marktsubstitute werden darum in diesem Kapitel übersichtsartig in ihren Allokationswirkungen diskutiert, um im nächsten Kapitel die Betrachtungsweise speziell auf die Vertragsvereinbarungen auszurichten.

In Zweiperiodenmodellen der Ressourcentheorie sind die Auswirkungen fehlender Zukunftsmärkte und die Reaktionen der Wirtschaftssubjekte, die als Substitute für die fehlenden Zukunftsmärkte langfristige Lieferverträge abschließen, Terminkontraktmärkte und Optionskontraktmärkte einrichten, bisher nicht analysiert worden.1)

Abschnitt 5.1. betrachtet die Ressourcenallokation ohne Zukunftsmärkte und ohne die Möglichkeit, Marktsubstitute einrichten zu können. Die folgenden Abschnitte erweitern die Analyse, indem sie dem Abbauunternehmen die Möglichkeit geben, seine Abbaumengen im voraus über langfristıge Lieferverträge zu verkaufen (Abschnitt 5.2:), seine Erträge aus dem Abbau durch Hedging auf dem Terminkontraktmarkt abzusichern (Abschnitt 5.3.) oder eine Verkaufoption auf dem Optionsmarkt zu erwerben ( $A b$ schnitt 5.4.).

1) Von Roberts (1980) gibt es eine Analyse des Angebotsverhaltens bei langfristigen Lieferverträgen in einem Mehrperiodenmodell. 


\subsection{Ressourcenabbau ohne Zukunftsmärkte}

Existieren keine Zukunftsmärkte, dann stehen eınem Abbauunternehmen für den Verkauf seines bekannten Ressourcenbestandes $R_{0}$ nur die Kassamärkte zur Verfügung. In einem Zweıperiodenmodell1) sind dies der Kassamarkt heute $(t=0)$ und der Kassamarkt morgen $(t=1)$. Aus heutiger Sicht ist der Preis auf dem Kassamarkt morgen eine Zufallsvariable $\mathbf{p}_{1}$, uber die sich der Abbauunternehmer Erwartungen bilde $p_{1} e=E\left(p_{1}\right)$, und deren Varianz $\sigma_{\mathrm{p}}^{2}$ betrage.

Der Gewinn aus der Abbautätigkeit über beide Perioden ist damit ebenfalls eine Zufallsvariable. Werde die Ressource ohne Abbaukosten gefördert und sei $\delta$ der Zinssatz der risikolosen Erlösanlage auf dem Kapitalmarkt, dann ergibt sich der Gewinn in Werten der Periode $t=1$ als

$$
\mathbf{G}=(1+\delta) p_{0} q_{0}+p_{1} q_{1}
$$

Ein nicht risikoneutraler Abbauunternehmer wird den Erwartungsnutzen seines Gewinnes uber der Ressourcenrestriktion $R_{0} \geq q_{0}+q_{1}$ maximieren. ${ }^{2}$ )

$$
\begin{aligned}
& \max _{\left(q_{0}, q_{1}\right)} E\left\{U\left[(1+\delta) p_{0} q_{0}+p_{1} q_{1}\right]\right\} \\
& \text { u.d.B. } \quad R_{0} \geq q_{0}+q_{1}, R_{0} \text { gegeben, } q_{0}, q_{1} \geq 0 .
\end{aligned}
$$

1) Vgl. Weinstein/Zeckhauser (1974) zu einem Erwartungsnutzenansatz oder Levy/Sarnat (1975) zu einem Mittelwert-Varianz-Ansatz, wo die Portfolioentscheidung eines ressourcenexportierenden "low absorbers" diskutiert wird.

2) $U(G)$ sei eine zweımal stetıg differenzierbare Nutzenfunktion. 
An notwendigen Bedingungen werden erhalten

(5.3)(i) $\quad E\left(U^{\prime}\right)(1+\delta) p_{0}-\lambda$

(5.3)(ii) $\quad E\left(U^{\prime} p_{1}\right)-\lambda$

$$
\left\{\begin{array}{cc}
=0 & q_{0}>0 \\
& \text { für } \\
\leq 0 & q_{0}=0
\end{array}\right.
$$$$
\begin{cases}=0 & q_{1}>0 \\ & \text { für } \\ \leq 0 & q_{1}=0\end{cases}
$$$$
\left\{\begin{array}{lll}
=0 & & \lambda>0 \\
& \text { für } & \\
\geq 0 & \lambda=0
\end{array}\right.
$$

(5.3)(iii) $\quad R_{0}-q_{0}-q_{1}$

Für positive Abbaumengen in beiden Perioden folgt aus (i) und (ii)

(5.4)(i) $\quad(1+\delta) p_{0}=\lambda E\left(U^{\prime}\right)-1$

(5.4)(ii) $\quad E\left(U^{\prime} p_{1}\right)=\lambda$.

Mit $\quad E\left(U^{\prime} p_{1}\right)=\left\{p_{1} e+\operatorname{cov}\left(U^{\prime}, p_{1}\right) E\left(U^{\prime}\right)-1\right\} E\left(U^{\prime}\right)$,

wird als Beziehung zwischen dem Kassapreis in $t=0$ und dem für $t=1$ erwarteten Kassapreis $p_{1}$ e erhalten

$$
\left.(1+\delta) p_{0}=p_{1} e+\operatorname{cov}\left(U^{\prime}, p_{1}\right) E\left(U^{\prime}\right)-1,1\right)
$$

1) Es ist $\operatorname{cov}\left(U^{\prime}, p_{1}\right)<0$ für eınen risikoaversen Abbauunternehmer. 
Im Maxımum des Erwartungsnutzens ist der Abbauunternehmer indifferent $z$ wischen einem Angebot in $t=0$ und einem Angebot in $t=1$. Denn es gilt 5.5: Der aufdiskontierte Kassapress von heute ist gleich dem um eine Risıkogroße korrigierten erwarteten Kassapreis von morgen.

Für das Angebotsverhalten ergibt sich aus den notwendigen Bedingungen

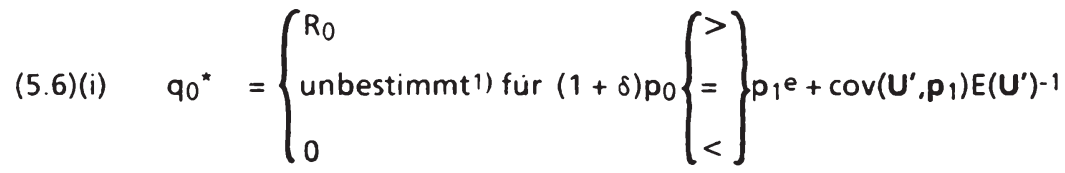
(5.6)(ii) $q_{1}^{*}=R_{0}-q_{0}^{*}$.

In der Portfoliotheorie werden die einzelnen Gewinnsituationen in Termini des Erwartungswertes und der Varianz des Gewinnes bewertet. Erwartungswert und Varianz gehen in die Zielfunktion ein und bilden das Kriterium für die Allokationsentscheidung:

$$
\begin{aligned}
q_{0}^{*}=R_{0}, q_{1}^{*}=0 \Rightarrow E(G) & =(1+\delta) p_{0} R_{0} \text { und } \\
\operatorname{var}(G) & =0 \\
q_{0}^{*}, q_{1}^{*}>0 \Rightarrow E(G) & =(1+\delta) p_{0} q_{0}{ }^{*}+p_{1} e_{1} q_{1}^{*} \text { und } \\
\operatorname{var}(G) & \left.=\left(R_{0}-q_{0}\right)^{*}\right)_{0}^{2}
\end{aligned}
$$

(5.7)(ii) $\quad 90^{*} .91^{*}>0$

(5.7)(iii) $q_{0}{ }^{*}=0, q_{1}^{*}=R_{0} \Rightarrow E(G)=p_{1} e R_{0}$ und

$$
\operatorname{var}(\mathbf{G})=R_{0} \sigma_{p^{2}} \text {. }
$$

1) Der Anbieter ist indifferent $z$ wischen dem Angebot von $R_{0}$ in $t=0$ oder in $t=1$. Die Nachfrage bestimmt die Aufteilung der Abbaumengen. 
Mit zunehmenden Handelsmengen auf dem zukünftigen Kassamarkt steıgt die Varianz des Gewinnes 1) aus der Abbautätıgkeit. Der Abbauunternehmer nimmt eine großßere Varianz seınes Gewınnes dann hın, wenn er mit größeren Gewınnerwartungen rechnen kann [5.7 (ii)].

Mit Tobins2) Konzept zur Liquiditàtspräferenz läßt sıch die Allokationsentscheidung des Abbauunternehmers näherungsweise ${ }^{3)}$ graphisch darstellen.

Für gegebene Werte $R_{0}, p_{0}, p_{1} e$, $\delta$ und $\sigma_{p}^{2}$ gibt Gleichung 5.8 maxımal erreichbare Kombinationen von Gewinnerwartungen und Gewinnvarianzen an.4)

$$
E(G)=(1+\delta) p_{0} R_{0}+\left[p_{1} e-(1+\delta) p_{0}\right] \sigma_{p^{-1}} \sqrt{\operatorname{var}(G)} .
$$

1) Definiert man das Risiko der Abbautätigkeit als var(G), so folgt für die

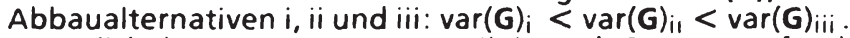

Bezüglich der Erwartungswerte gilt je nach Erwartung für den zukünftigen Kassamarktpreis:

2) Tobin (1958), $S .72$. $E(\mathbf{G})_{i}><E(G)_{1 i}><E(G)_{i i i} \Leftrightarrow(1+\delta) p_{0}><p_{1} e$.
Bei Tobin wird eine Funktion der $\operatorname{Art} U[E(G), \operatorname{var}(G)]$ verwendet mit $\partial U / \partial E(G)>0, \partial U / \partial \operatorname{var}(\mathbf{G})<0$ für Risikoaversion ( $>0$ für Risikofreude), $\partial 2 \mathrm{U} / \partial \mathrm{E}(\mathbf{G})^{2}<0, \partial 2 \mathrm{U} / \partial \operatorname{var}(\mathbf{G})^{2}><0, \partial 2 \mathrm{U} / \partial \mathrm{E}(\mathbf{G}) \partial \operatorname{var}(\mathbf{G})<0$ für Risikoaversion ( $>0$ für Risikofreude).

Markowitz (1959) betrachtet als Zielfunktion $Z=E(G)+\lambda \operatorname{var}(G)$. Hierin steht $\lambda<0$ fur das Ausmaß der Risikoaversion. Sowohl bei Tobin als auch bei Markowitz gilt es, für die optimale Entscheidung die Zielfunktion zu maximieren.

3) Die Äquivalenz des Optimierungskalküls 5.2 und desjenigen, wie es dem Schaubild nach Tobin zugrundeliegt,

$\max U[E(G), \operatorname{var}(G)]$

$\left(q_{0}, q_{1}\right)$

u.d.B. $E(G)=(1+\delta) p_{0} R_{0}+\left[p_{1} e-(1+\delta) p_{0}\right] \sigma_{p^{-1}} \sqrt{\operatorname{var}(G)}, q_{0}, q_{1} \geq 0$,

gilt nur für quadratische Nutzenfunktionen. Siehe hierzu Arrow (1970), S.29:"The characterization of probability distributions by mean and variance would be valid, at least in a special case, under the present theory, if the utility functions of income were quadratic,...". Vgl. auch Sinn (1980b), S.126f.

4) Dies wird erhalten aus 5.7 (ii) mit $R_{0}=q_{0}+q_{1}$. 
Tobın bezeıchnet diesen Zusammenhang als "Budgetgerade" eines eine Portfolioentscheidung durchführenden Individuums. ") Die Varianz des Gewınnes hängt über die Abbaumengen von morgen dırekt ab von der Varianz des zukünftigen Kassapreıses. Um zu eıner Allokationsentscheidung zu kommen, hat man die "Budgetgerade" einer Indifferenzkurvenschar gegenüberzustellen, die Kombinationen von Gewinnerwartungen und Gewinnvarıanzen bewertet. Gewählt wird jene Kombination von $\mathrm{q}_{0}$ und $q_{1}$, die uber Gleichung 5.8 den maximalen Nutzenindex realisiert. Schaubild 5.12) stellt dieses Konzept dar.

In der oberen Hälfte des Schaubildes ist die Budgetgerade ${ }^{3)}$ gemäß Gleichung 5.8 einer Indifferenzkurve gegenübergestellt, die nach Tobin charakterıstisch für eın diversifizierendes Wirtschaftssubjekt ist.4) Gegenuber einer Erhöhung der Standardabweichung des Gewinnes bleibt das Wirtschaftssubjekt indifferent, wenn es mit eıner Steigerung des erwarteten Gewinnes um mehr als eine Einheit entlohnt wird.

1) Levy/Sarnat (1975), S.363 sprechen hier von einer "Transformationskurve" zwischen risikobehafteten und sicheren Vermögenswerten.

2) Die Darstellungsweise in der rechten Diagrammhälfte findet man bei Levy/Sarnat (1975), S.364.

3) Die Budgetgerade endet in B. Ihr Definitionsbereich wird bestimmt durch die möglichen Standardabweichungen im Intervall $\left[0, R_{0} \sigma_{p}\right]$.

4) Bezeichne $E=E(G)$ den Erwartungswert, $V=\operatorname{var}(G)$ die Varianz des Gewinnes und der Index $E$ bzw. V die partielle Ableitung einer Nutzenfunktion nach dieser Yariablen. Unter den Annahmen, für die der Indifferenzkurve I zugrundeliegende Nutzenfunktion $U(E, V)$ (siehe Fußnote 2 S.129) ergibt sich für die Steıgung der Indifferenzkurve: $d E / d V=-U_{V} / U_{E}>0$ für Risikoaversion ( $<0$ für Risikofreude); $\mathrm{d}^{2} \mathrm{E} / \mathrm{dV} 2=-\left(U_{E} U_{V V}-U_{V} U_{E V}\right) U_{E^{-2}}>0$ für abnehmenden und konstanten Grenznutzen des Risikos. 
Schaubild 5.1: Die Allokationsentscheidung im Portfolioansatz

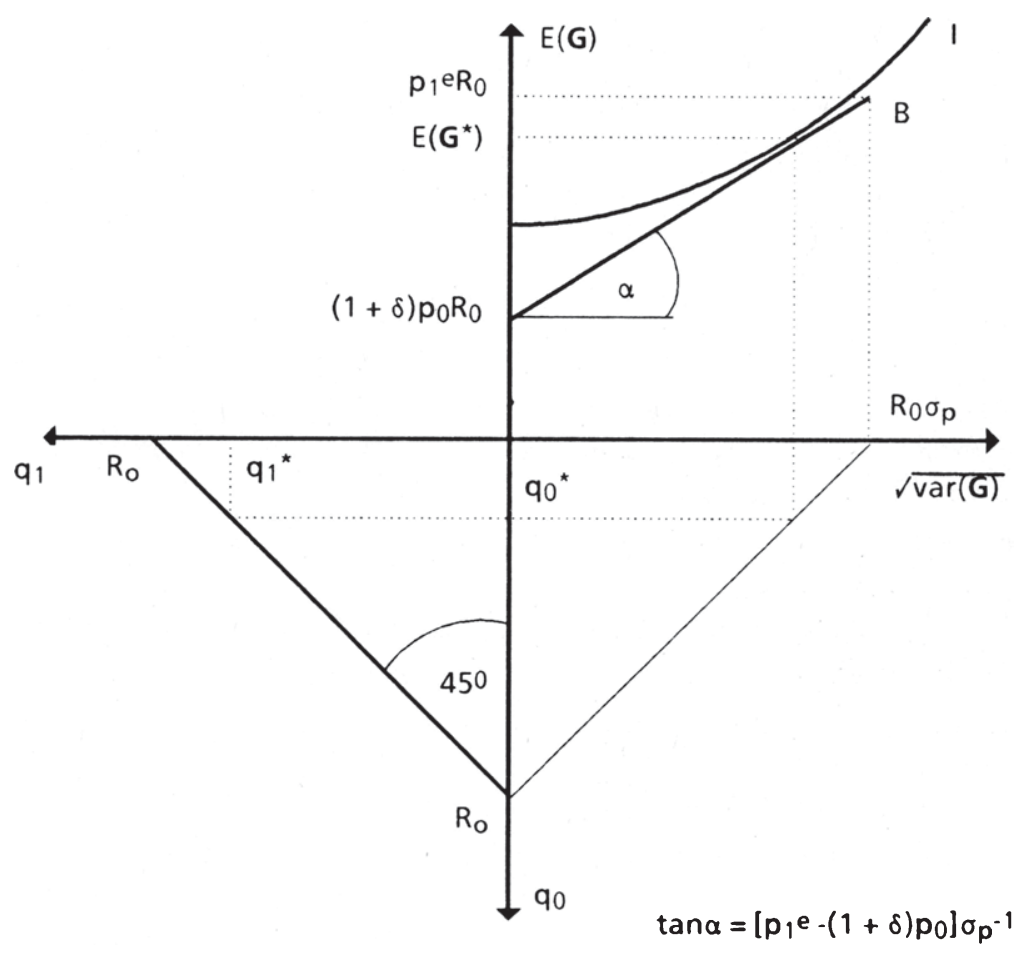

Aus den notwendigen Bedingungen 5.6 folgt für das Angebot $q_{0}{ }^{*}, q_{1}{ }^{*}>0$ eines risikosaversen Abbauunternehmens die Gültigkeit von $p_{1} e>$ $(1+\delta) p_{0}, d$.h. die Budgetgerade hat eine positive Steigung. Für einen risikoneutralen (bzw. risikofreudigen) Abbauunternehmer würde gelten $p_{1} e$ $=(1+\delta) p_{0}\left[\right.$ bzw. $\left.p_{1} e<(1+\delta) p_{0}\right]$.

Die untere rechte Hälfte des Schaubildes enthält für gegebenes $R_{0}$ die Darstellung der Standardabweichung des Gewinnes in Abhängigkeit von den 
Abbaumengen für den Kassaverkauf qo (Gleichung 5.7 (ii) letzte Zeile). Hierfür soll dıe Bezeıchnung Risıkogerade gelten.

Links daneben wırd über die Ressourcenbestandsrestriktion die Angebotsmenge $q_{1}$ als Restgroße bestimmt.

Der Tangentialpunkt zwischen der Indifferenzkurve I und der Budgetgeraden bestimmt die nutzenoptimale Aufteilung zwischen dem Kassaverkauf des Ressourcenbestandes heute $\mathrm{q}_{0}{ }^{*}$ und der Ressourcenbestandshaltung für den morgigen Kassaverkauf $q_{1}{ }^{*}=R_{0}-q_{0}{ }^{*}$.

Hat der risikoaverse Abbauunternehmer eine Preiserwartung $p_{1} e=(1+\delta) p_{0}$, (die Budgetgerade verläuft waagerecht), dann wird er seinen gesamten Ressourcenbestand in $t=0$ anbieten. Der risikoneutrale $A b$ bauunternehmer wäre bei dieser Preiserwartung indifferent zwischen einem Angebot in $t=0$ und $t=1$. Der risikoaverse Abbauunternehmer fordert für ein positives Angebot in $t=1$ eine Wachstumsrate des Preises von mehr als $1+\delta$. Gemessen an dem Abbauverhalten des risikoneutralen Abbauunternehmers würde der risikoaverse Abbauunternehmer die Ressource "unterkonservieren", 1) $d$.h. vermehrt in $t=0$ abbauen.

Ein risikofreudiger Abbauunternehmer würde bei der Preiserwartung $p_{1} e=(1+\delta) p_{0}$ seinen gesamten Bestand in $t=1$ anbieten. Damit er sein Angebot auf beide Perioden verteilt, darf der Ressourcenpreis nur mit einer Rate kleiner als $1+\delta$ wachsen.2) Vergleicht man den risikofreudigen Abbauunternehmer in seinem Abbauverhalten wieder mit einem risikoneutralen, dann ist das risikofreudige Abbauverhalten als "überkonservierend" zu bezeichnen, $d$.h. es wird verstärkt in $t=1$ abgebaut.

1) Vgl. zu diesem Ergebnis Weinstein/Zeckhauser (1975), S.383.

2) Aus den notwendigen Bedingungen 5.6 folgt für den risikofreudigen Abbauunternehmer $p_{1} e<(1+\delta) p_{0}$ für $q_{0}, q_{1}>0$. Die Budgetgerade hat somit eine negative Steigung. Für die Indifferenzkurve gilt $\mathrm{dE} / \mathrm{d} \sqrt{\operatorname{var}(G)}<0$ und je nach Verlauf des Grenznutzens für das Rısiko $d^{2} E / d(\sqrt{\operatorname{ar}(G)})^{2}><0$. 
Die Auswirkungen einer Variation des erwarteten Rohstoffpreises $p_{1}$ e oder einer Variation der Standardabweichung dieses Preises $\sigma_{p}$ auf die Allokationsentscheidung eines risıkoaversen Abbauunternehmers lassen sich in Schaubild 5.1 leicht darstellen: Mit zunehmendem Erwartungswert wird bei gleicher Varianz des Preises $p_{1}$ mehr in Periode 1 angeboten. Mit zunehmender Standardabweichung $\sigma_{p}$ wird bei gleichem Erwartungswert weniger in der Periode 1 angeboten. 1)

Neben dem Angebotsverhalten des "diversifizierenden" risikoaversen Abbauunternehmers sind noch weitere Angebotsverhalten ableitbar, wenn man eınen risikofreudigen Abbauunternehmer unterstellt, bzw. einen von Tobin als "Plunger" handelnden Abbauunternehmer annımmt. 2)

Der risikofreudige Abbauunternehmer ist zu kennzeichnen mit3) $d U / d E(G), d U / d \operatorname{var}(G), d 2 U / d E(G) \operatorname{var}(G)>0$ und $d 2 U / d \operatorname{var}(G)^{2}>0$.

Das Verhalten des "Plungers" ist bestimmt durch $d U / d E(G)>0, d U / d \operatorname{var}(G), d^{2} U / d E(G) \operatorname{var}(G)<0$ und $d^{2} U / d \operatorname{var}(G)^{2} \geq 0$.

Bei gegebener Preiserwartung $p_{1} e>(1+\delta) p_{0}$ für Risikoaversion und $p_{1} e<(1+\delta) p_{0}$ für Risikofreude, erhält man für beide Arten des Risikoverhaltens sowohl eine diversifizierende als auch eine "Entweder-oder" Entscheidung.

1) Mit zunehmendem Erwartungswert vergroßert sich das Steigungsmaß der Budgetgeraden; mit zunehmender Standardabweichung verringert es sich.

2) Vgl. Tobin (1958), S.78.

3) Vgl. Fußnote 2 S.129. 
Schaubild 5.2: Die Allokationsentscheidung bei unterschiedlichem Risikoverhalten
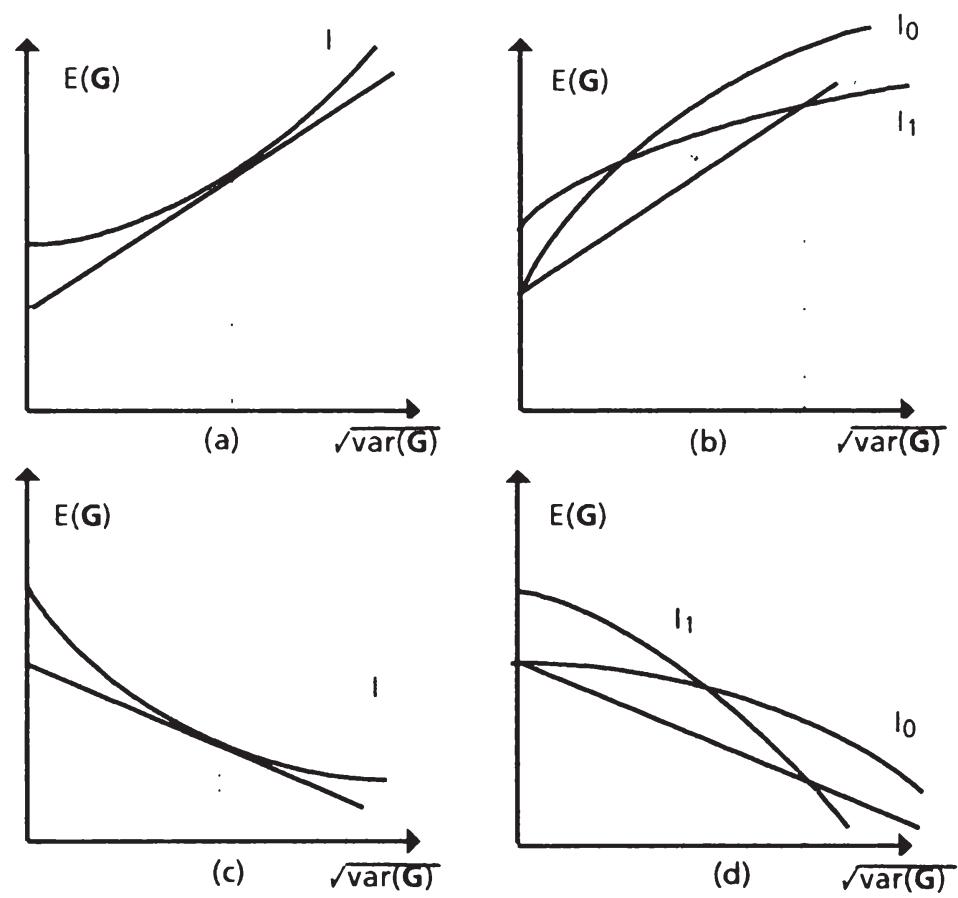

Schaubild 5.2 (a) zeigt nochmals den bisher diskutierten Fall des risikoaversen, diversifizierenden Abbauunternehmers. Darunter in (c) ist der risikofreudige, diversifizierende Rohstoffbesitzer dargestellt. Das Verhalten eines Plungers unter Risikoaversion und das eines Plungers unter Risikofreude ist in (b) und (d) gegenübergestellt. Die Indifferenzkurven $l_{1}$ sind gegenüber den Indifferenzkurven Io durch stärker zunehmenden Grenznutzen 
des Risikos gekennzeichnet. Bei stark zunehmendem Grenznutzen des Risikos $\left(I_{1}\right)$ wird ein Plunger den gesamten Ressourcenbestand auf dem Kassamarkt in $\mathrm{t}=1$ anbieten wollen. Im Falle der Indifferenzkurve 10 wird geplant, den Ressourcenbestand gänzlich auf dem heutigen Kassamarkt anzubieten.

Damit sowohl in $\mathrm{t}=0$ als auch in $\mathrm{t}=1$ ein positives Rohstoffangebot zustandekommt und sich Marktgleichgewichte bei positiven Preisen und Mengen einstellen, hat man die Abbauunternehmer als "diversifizierend" anzunehmen.

Für die Bestimmung der Marktgleichgewichte sei deshalb unterstellt:

$\mathrm{N}$ risikoaverse Ressourcenbesitzer bieten die Ressource aus ihren Beständen $R_{0 i}$ an. Die Angebotsmengen $q_{0 i}$ und $q_{1 i}$ des $i$-ten Abbauunternehmers in $t=0,1$ seien Maximierer des repräsentativen Kalküls 5.2. Das gesamte Angebot auf gegenwärtigem und zukünftigem Kassamarkt wird erhalten als

$$
q_{0^{a}}=\sum_{1=1} q_{\text {oi }} \text { bzw. } R_{0}-q_{0^{a}}=\sum_{1=1 N} q_{1 i},
$$

mit dem gesamten abbaubaren Ressourcenbestand

$$
R_{0}=\Sigma_{1=1 N} R_{\text {oi }} .
$$

Die Nachfrage in $\mathrm{t}=0$ sei

$$
p_{0}=N_{0}\left(q_{o^{n}}\right)
$$

und in $\mathrm{t}=1$ gelte für die Nachfrage

$$
\mathbf{p}_{1}=N_{1}\left(q_{1}{ }^{n}\right) u_{1} \text { mit } E\left(u_{1}\right)=1 \text {. }
$$

Den Abbauunternehmen werden hinsichtlich des Preises $\boldsymbol{p}_{1}$ rationale Erwartungen unterstellt, $d$.h. ihre subjektive Erwartung $p_{1}$ e stımmt überein mit $E\left(p_{1}\right)=N_{1}\left(q_{1} n\right)$.

$$
p_{1} e=N_{1}\left(q_{1} n\right) \text {. }
$$

Unter diesen Annahmen herrscht Gleichgewicht auf den beiden Markten dann, wenn gilt 
(5.14) $\quad q_{0^{a}}=q_{0^{n}}$ und $R_{0}-q_{0}^{a}=q_{1}^{n}$.

Beıde Märkte sind geräumt, der Ressourcenbestand wird in $t=1$ erschöpft.

Für die Gleıchgewichtspreise $p_{0}$ und $p_{1}$ e gilt die Bezıehung

$$
(1+\delta) p_{0}=p_{1} e+\operatorname{cov}\left(U^{\prime}, p_{1}\right) E\left(U^{\prime}\right)^{-1}
$$

Ausgehend von den notwendigen Bedingungen 5.3 (i) bis (iii) als Bestimmungsgleichungen für das Verhalten der Angebotsseite und den unterstellten Nachfragen für $t=0,1$, führt eine komparative Analyse zu den folgenden Ergebnissen: 1$)$

Tabelle 5.1: Ergebnisse einer komparativen Analyse

\begin{tabular}{c|ccc} 
& $\mathrm{q}_{0}$ & $\mathrm{q}_{1}$ & $\lambda$ \\
\hline$\delta$ & + & - & $+1-$ \\
$\mathrm{R}_{0}$ & $+1-$ & $+1-$ & $+1-$ \\
$\operatorname{cov}\left(\mathrm{U}^{\prime}, \mathbf{p}_{1}\right)$ & - & + & $+1-$
\end{tabular}

1) Siehe Anhang IV. Die Elemente der Tabelle sind zu lesen als Vorzeichen der Differentıalquotienten aus endogenen und exogenen Variablen; z. Bsp. dqo $/ d \delta>0$. Eindeutige Vorzeichen gelten nur für Gleichgewichte in bestimmten Bereichen der Nachfrage. 
Eine Steigerung der Diskontrate und eine Erhöhung des Risikos führen tendenziell zu der gleichen Reallokation der Ressourcen.

Die Auswirkungen einer höheren Diskontrate sınd die gleıchen wie in Abschnitt 2.4, wo im Mehrperiodenmodell unter Sicherheit das Ergebnis in Form eines kürzeren Abbauzeitraumes diskutiert wurde. Die Abbaumengen der Gegenwart ( $q_{0}$ ) steigen, die Abbaumengen der Zukunft $\left(q_{1}\right)$ sinken.

An ein höheres Risiko [ $\operatorname{dcov}\left(U^{\prime}, \mathbf{p}_{1}\right)<0$ ] passen sich die Abbauunternehmer an, indem sie ihr Abbauprogramm ebenfalls zugunsten der heutigen Fördermengen umverteilen.

Eindeutige Auswirkungen einer Erhöhung des Ressourcenbestandes werden erhalten, wenn in den Gleichgewichten der Ausgangsstituation galt: $n_{0}=p_{0}\left(N_{0} q_{0}\right)^{-1}>-1$ und $\eta_{1} e=p_{1} e\left(N_{1}^{\prime} q_{1}\right)^{-1}$. Der Zuwachs des Ressourcenbestandes wird auf beide Perioden verteilt.

Die eben genannten Bedingungen sind nicht hinreichend für eine eindeutige Aussage über die Auswirkungen der parametrischen Variationen von $\delta, R_{0}$ und $\operatorname{cov}\left(U^{\prime}, p_{1}\right)$ auf $\lambda$. 


\subsection{Ressourcenabbau mit "langfristigen" Verträgen}

Dem in Abschnitt 5.1. beschriebemen repräsentativen Abbauunternehmer soll nun zur Minderung des Risikos1) die Möglichkeit gegeben sein, langfristige Lieferverträge 2 ) für den zukünftigen Abbau abschließen zu können. Er hat damit drei Erlösalternativen des Rohstoffverkaufs: Auf dem heutigen Kassamarkt erhält er den Preis $p_{0}$, auf die Zusage vertraglicher Lieferungen für morgen erhält er morgen den Preis $p_{v}$ und für den morgigen Kassaverkauf erwartet er einen Preis $p_{1}$ e mit der Varianz $\sigma p^{2}$.

Neben der Ressourcenbestandshaltung von heute auf morgen hat der Abbauunternehmer als Alternative die Anlage seines Erlöses auf dem Kapitalmarkt, der ihm zum Zins $\delta$ einen sicheren Ertrag liefert.

Der gesamte Erlös in Werten der zweiten Periode ergibt sich unter diesen Annahmen zu:

$$
\mathbf{G}=(1+\delta) p_{0} q_{0}+p_{v} q_{v}+p_{1} q_{1} .
$$

$q_{v}$ steht für die vertraglich zu liefernden Rohstoffmengen.

Für risikoaverse bzw. risikofreudige Abbauunternehmer lautet das Optimierungsproblem

$$
\begin{aligned}
& \max _{\left(q_{0}, q_{1}\right)} E\left\{U\left[(1+\delta) p_{0} q_{0}+p_{v} q_{v}+p_{1} q_{1}\right]\right\} \\
& \text { u.d.B. } \quad R_{0} \geq q_{0}+q_{v}+q_{1}, R_{0} \text { gegeben, } q_{0}, q_{v}, q_{1} \geq 0 .
\end{aligned}
$$

1) Vgl. Fußnote 1 S.129.

2) Die "lange Frist" bezieht sıch hier auf die "gesamte "Zukunft, die eine Periode ausmacht. 
An notwendigen Bedingungen folgen

(5.18)(i) $\quad E\left(U^{\prime}\right)(1+\delta) p_{0}-\lambda$

(5.18)(ii) $\quad E\left(U^{\prime} p_{v}\right)-\lambda$

(5.18)(iii) $E\left(U^{\prime} p_{1}\right)-\lambda$

(5.18)(iii) $R_{0}-q_{0}-q_{v}-q_{1}$

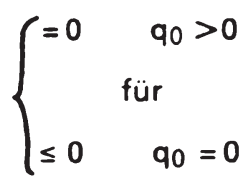$$
\left\{\begin{array}{cc}
=0 & q_{v}>0 \\
& \text { für } \\
\leq 0 & q_{v}=0
\end{array}\right.
$$$$
\begin{cases}=0 & q_{1}>0 \\ & \text { für } \\ \leq 0 & q_{1}=0\end{cases}
$$$$
\left\{\begin{array}{lll}
=0 & & \lambda>0 \\
& \text { für } & \\
\geq 0 & \lambda=0 .
\end{array}\right.
$$

Für positive Angebotsmengen $q_{0}, q_{v}, q_{1}>0$ ergibt sich

(5.19)(i) $\quad(1+\delta) p_{0}=\lambda E\left(U^{\prime}\right)-1$

(5.19)(ii) $\quad p_{v}=\lambda E\left(U^{\prime}\right)-1$

(5.19)(iii) $p_{1} e+\operatorname{cov}\left(U^{\prime}, p_{1}\right) E\left(U^{\prime}\right)^{-1}=\lambda E\left(U^{\prime}\right)^{-1}$.

Der Abbauunternehmer ist zwischen diesen drei Alternativen des Rohstoffangebotes indifferent, falls gilt

(5.20) $\quad(1+\delta) p_{0}=p_{v}=p_{1} e+\operatorname{cov}\left(U^{\prime}, p_{1}\right) E\left(U^{\prime}\right)^{-1}$ 
Ein risikoaverser Abbauunternehmer wird wie folgt handeln:

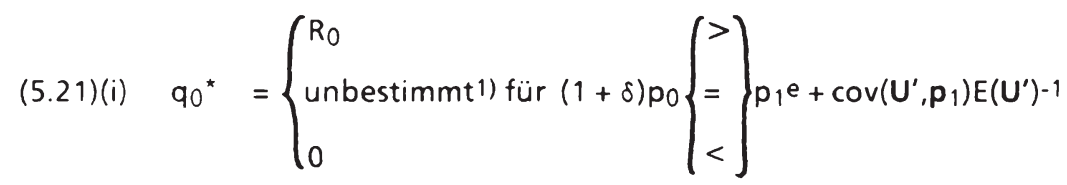

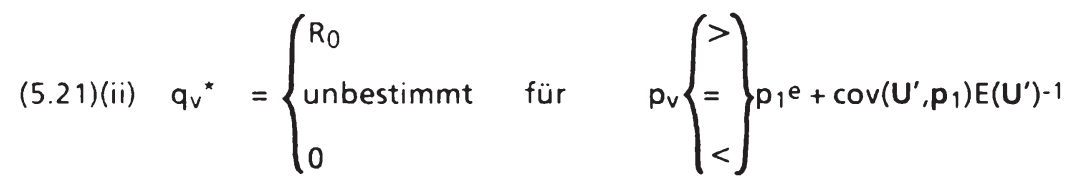

(5.21)(iii) $q_{1}^{*}=R_{0}-q_{0}^{*}-q_{v}^{*}$.

Stellt man die Gleichheiten in 5.20 der Arbitragebedingung gegenüber wie sıe in eınem Zweiperiodenmodell mit vollkommenem Zukunftsmarkt auftritt, $(1+\delta) p_{0}=p_{1}$, dann ist zu sehen, daß der Vertragspreis $p_{v}$ die Allokationsfunktion des Presses $p_{1}$ übernimmt. Der Preis der Ressource wächst in diesem Modell trotz fehlenden Zukunftsmarktes von $p_{0}$ auf $p_{v}$ mit der Rate $\delta$ und allokiert damit die Ressource zwischen den beiden Zeitpunkten ebenso wie bei Existenz eines Zukunftsmarktes.

Zur portfoliotheoretischen Betrachtungsweise seien für den Abbauunternehmer wieder drei Gewinnsituationen mit zunehmender Varianz beschrieben:

1) Die Nachfrageseite bestımmt die Allokation. 
(5.22)(i) $q_{0}{ }^{*}, q_{v}{ }^{*}>0$ mit $R_{0}=q_{0}{ }^{*}+q_{v}^{*} ;$ es gilt $(1+\delta) p_{0}=p_{v}$

$$
\begin{aligned}
\Rightarrow E(G) & =p_{v} R_{0} \text { und } \\
\operatorname{var}(\mathbf{G}) & =0
\end{aligned}
$$

(5.22)(ii) $q_{0}{ }^{*}, q_{v}{ }^{*}, q_{1}{ }^{*}>0$; es gelten die Gleichheiten in 5.20 ,

$$
\begin{aligned}
\Rightarrow E(G) & =p_{v}\left(q_{0}{ }^{*}+q_{v}{ }^{*}\right)+p_{1} e_{1} q^{*} \text { und } \\
\operatorname{var}(G) & =\left(R_{0}-q_{0}{ }^{*}-q_{v}{ }^{*}\right)^{2} \sigma_{p^{2}}
\end{aligned}
$$

(5.22)(iii) $q_{0}^{*}=0, q_{1}^{*}=R_{0} \Rightarrow E(G)=p_{1} e R_{0}$ und

$$
\operatorname{var}(\mathbf{G})=R_{0} \sigma_{\mathbf{p}}{ }^{2}
$$

Die Indifferenz des Abbauunternehmers im Fall (ii) läßt keine Aussage zu, ob die Vertragsabschlüsse zu einer Verringerung der Varianz des Gewinnes führen (Vergleich von 5.7 (ii) mit 5.22 (ii)). Erst wenn man in einem Marktgleichgewicht bestimmt hat, daß das $90^{*}$ gemäß 5.7 (ii) kleiner als die Summe $90^{*}+q_{v}^{*}$ gemäß 5.22 (ii) ist, hat man die Behauptung bewiesen, daß die Existenz von Verträgen risikomindernd ") wirkt.

Schaubild 5.3 zeigt in portfoliotheoretischer Darstellungsweise die Allokationsentscheidung des Abbauunternehmers zwischen dem Angebot heute qo und dem Angebot für morgen über Verträge bzw. den zukünftigen Kassamarkt.

Die Budgetgerade des Abbauunternehmers lautet ähnlich Gleichung 5.8. Im Unterschied zu ihr kann nun bei Indifferenz zwischen den Absatzarten alternativ $(1+\delta) p_{0}=p_{v}$ gesetzt werden.

1) Risikomindernd im Sinne kleinerer Varianzen für den optimalen Wert einer Zielfunktion (Gewinn). Auch hier gilt, daß von I nach ii nach ii! die Erwartungswerte und die Varianzen des Gewinnes zunehmen. 


$$
E(G)=p_{v} R_{0}+\left(p_{1} e-p_{v}\right) \sigma_{p}-1 \sqrt{\operatorname{var}(G)}
$$

Das Schaubild ermóglicht nur die Darstellung der erwartungsnutzenoptimalen Aufteilung des "Portfolios". auf sichere und risikobehaftete Anlagealternativen; hier also die Darstellung der Aufteilung zwischen dem zukünftigen Kassaverkauf $q_{1}$ und dem heutigen Kassa- bzw. Vertragsverkauf $q_{0}+q_{v}$. Die Aufteilung des Rohstoffangebotes zwischen diesen beiden Absatzarten bleibt unbestimmt, da sie beide gleich sicher sind.

Für die diagrammatische Darstellung wird von einer gegebenen Vertragsmenge $q_{v}{ }^{*}$ ausgegangen. ${ }^{1)}$

Dem Abbauunternehmer sei wieder ein risikoaverses Verhalten unterstellt, d.h. $p_{1} e>p_{v}, b z w . p_{1} e>(1+\delta) p_{0}$, für positive Angebotsmengen auf den Kassamàrkten in $t=0,1$ und über Verträge.

Die Interpretation kann der in Abschnitt 5.1. gegebenen folgen mit dem Unterschied, daß anstelle von $q_{0}{ }^{*}$ nun $q_{0}{ }^{*}+q_{v}{ }^{*}$ als sichere Absatzmöglichkeit zu berücksıchtigen ist. Vor und nach der Einführung von Verträgen wird der Abbauunternehmer dieselbe Aufteilung zwischen risikofreiem und risikobehaftetem Rohstoffangebot vornehmen. Der Abschluß von Verträgen $q_{v}{ }^{*}$ wird das Angebot für den Kassamarkt $q_{0}{ }^{*}$ in $t=0$ verringern. Da das für den Kassamarkt in $t=1$ bereitgehaltene Angebot $q_{1}{ }^{*}$ unverändert bleibt, steigt mit den Vertragsabschlüssen die für $t=1$ geplante Abbaumenge $q_{v}{ }^{*}+q_{1}{ }^{*}$. In dieser Interpretation mindern die Vertragsabschlüsse insofern das Rısiko, als bei nun erhöhtem Rohstoffangebot in $t=1$ die Varianz des Gewinnes nicht gestiegen ist.

1) Wählt man diese Vorgehensweise für die graphısche Darstellung, dann hat man schon den Wertebereich von $\operatorname{var}(\mathbf{G})$ eingeschränkt. Es ist $0 \leq \operatorname{var}(\mathbf{G}) \leq\left(R_{0}-q_{v}{ }^{*}\right) 2 \sigma_{p}{ }^{2}$. 
Schaubild 5.3: Die Allokationsentscheidung mit "langfristıgen" Vertràgen

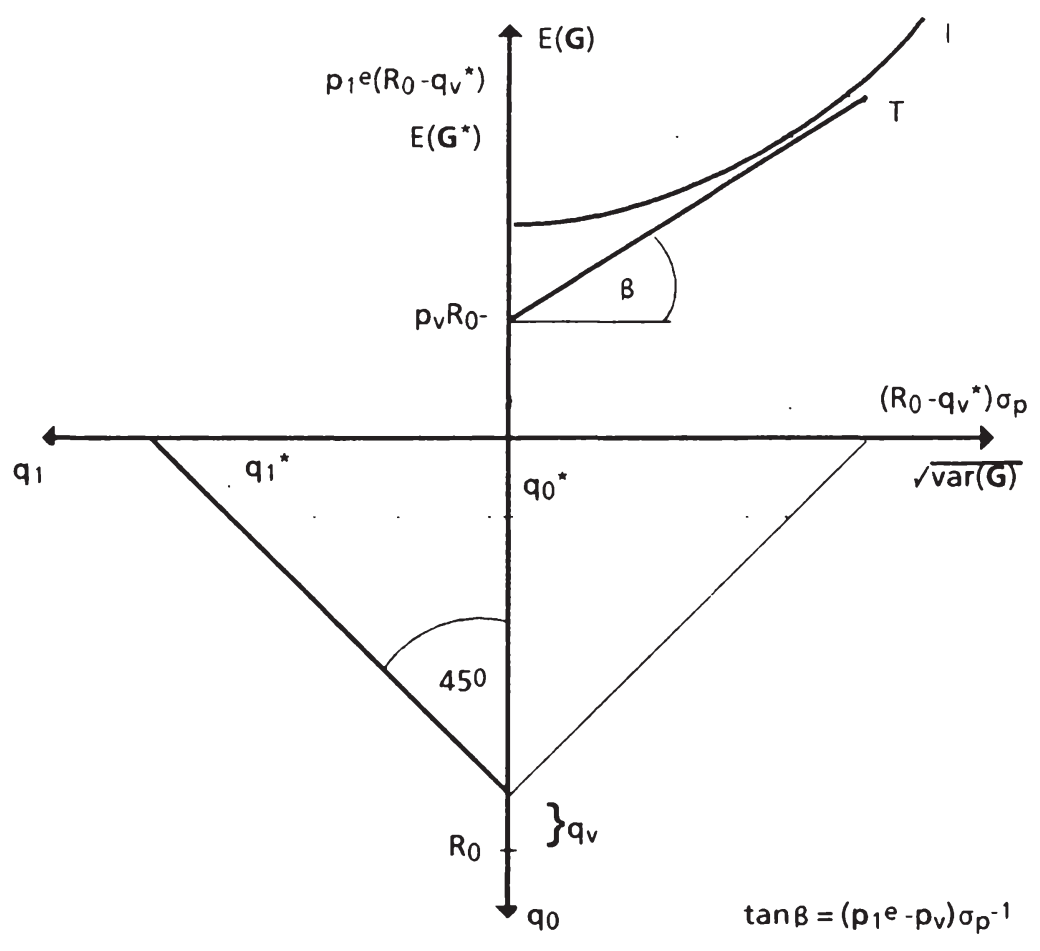

Bevor der Marktprozeß beschrieben wird, der die Ressourcen zwischen $t=0$ und $\mathrm{t}=1$ verteilt, soll das Gleichgewicht bestimmt werden.

Die Angebotsseite setze sich wieder aus $\mathrm{N}$ risikoaversen Unternehmen zusammen die ihre bekannten Ressourcenbestände $R_{0 i}$ abbauen. Die Angebotsmengen $q_{01}, q_{v 1}$ und $q_{11}$ seien Maximierer des Kalküls 5.17, so daß das Angebot in $\mathrm{t}=0$ bzw. $\mathrm{t}=1$ lautet

(5.24) $\quad q_{0}^{a}=\Sigma_{:=1} N q_{01}, q_{v}^{a}=\Sigma_{1=1} N q_{v 1}$ und $R_{0}-q_{0} a-q_{v} a=\Sigma_{1=1} N q_{1 i}$ 
mit

$$
R_{0}=\Sigma_{1=1} \quad R_{01}
$$

In $t=0$ gelte für die Nachfrage auf dem Kassamarkt

$$
p_{0}=N_{0}\left(q_{0} n\right)
$$

Für die in $t=1$ geltende Kassamarktnachfrage sei angenommen

$$
p_{1}=N_{1} k(q, n) u_{1} \text { mit } E\left(u_{1}\right)=1
$$

Verglichen mit der in 5.12 unterstellten Nachfrage $N_{1}$ ist in $N_{1} k$ der Nachfrageausfall aufgrund vertraglicher Liefermengen zu berücksichtigen. 1) Mit der Annahme zur Nachfrage nach Lieferungen über Verträge

$\left(5.27^{\prime}\right) \quad p_{v}=N_{v}\left(q_{v} n\right)$

kann für den Zusammenhang der Nachfragen $N_{1}$ und $N_{1} k$ in einfachster Form unterstellt werden

$\left(5.27^{\prime \prime}\right) \quad N_{1}^{-1}=\left(N_{1} k\right)^{-1}+N_{v^{-1}}$.

Besitzen die Anbieter rationale Erwartungen über den Preis auf dem zukünftigen Kassamarkt

$$
p_{1} e=N_{1} k\left(q_{1} n\right) \text {, }
$$

dann herrscht Gleichgewicht auf den Märkten, wenn gilt

$$
\begin{aligned}
& q_{0^{a}}=q_{0} \text { und } q_{v^{a}}=q_{v} n \text { in } t=0, \text { und } \\
& R_{0}-q_{0^{a}}-q_{v^{a}}=q_{1} a \text { in } t=1
\end{aligned}
$$

1) Vgl. auch Abschnitt 6.1.1. 
Zwischen den Gleichgewichtspreısen gelten die Bezıehungen

$$
(1+\delta) p_{0}=p_{v}=p_{1} e+\operatorname{cov}\left(U^{\prime}, p_{1}\right) E\left(U^{\prime}\right)^{-1} .
$$

Im Zeitpunkt $\mathrm{t}=0$ wird jeder Abbauunternehmer zunachst aufgrund seiner Erwartungshaltung und seiner Praferenzstruktur den Ressourcenbestand aufteilen in Abbaumengen, die ihm eınen sicheren Erlos bringen $\left(q_{0}+q_{v}\right)^{*}$ und Abbaumengen, die ihm einen risikobehafteten Erlos bringen $q_{1}{ }^{*}$. Die intertemporale Verteilung liegt damit noch nicht fest. Erst nachdem sich auf dem Kassamarkt in $t=0$ und für die Verträge Gleichgewichte derart gebildet haben, daß die Gleıchheit $(1+\delta) p_{0}=p_{v}$ gilt, liegt auch die intertemporale Verteilung in $q_{0}{ }^{*}$ und $q_{v}{ }^{*}+q_{1}{ }^{*}$ fest.

Im Gleichgewicht wächst der Rohstoffpreıs des Kassamarktes in $\mathrm{t}=0$ mit einer Rate größer als $1+\delta$ auf den erwarteten Preis des Kassamarktes in $t=1$. Der Preis für vertragliche Liefermengen hingegen wächst mit der Rate $1+\delta$. Die geringere Preissteigerung für Vertragsmengen ist zu begrunden mit dem fehlenden Risiko. Aufgrund des sicheren Ertrages $p_{v}$ pro Rohstoffeinheit ist der Abbauunternehmer bereit, eine geringere Preissteigerung hinzunehmen.

Da die Preissteigerung auf dem Kassamarkt mehr als $1+\delta$ beträgt, wird der risikoaverse Abbauunternehmer auch bei Vertragsabschlussen die Ressource unterkonservieren. Jedoch ist das Maß der Unterkonservierung hier, geringer als im vorigen Abschnitt, denn für die heute eingegangenen Lieferverpflichtungen für $t=1$ müssen Rohstoffmengen in Form von Reserven bereitgehalten werden.

Was kann über die Preisbewegungen und die damit verbundenen Reallokationsprozesse aufgrund der Einführung von Verträgen ausgesagt werden?

Für den zukünftigen Kassamarkt wird zunächst unverändert die Menge $q_{1}$ als Angebot bereitgehalten. Auf dem heutigen Kassamarkt wird das Angebot zugunsten der vertraglichen Lieferungen $q_{v}$ für $t=1$ reduziert. Die Nachfrage nach Rohstoffen für $t=0$ wird von den Vertragslieferungen nicht beeinflußt. Einfluß haben diese dagegen auf die Nachfrage nach Rohstoffen auf dem Kassamarkt in $t=1$. Dort geht die Nachfrage zurück. Von 
der Angebotsseite hat man in $\mathrm{t}=0$ eıne Tendenz zur Erhöhung des Preises $p_{0}\left(\right.$ für $\left.(1+\delta) p_{0}<p_{v}\right)$. Über die Nachfrageseite erhalt man $\cap t=1$ eine Tendenz zur Senkung des erwarteten Preises $p_{1} e$. Als Reaktıon darauf werden die Abbauunternehmer eine Umverteilung des Angebots vom Kassamarkt in $\mathrm{t}=1$ auf den Kassamarkt in $\mathrm{t}=0$ vornehmen. Dort ist das über die Vertragsabschlüsse zustande gekommene Angebotsdefizit auszugleichen. Am Ende der Anpassungsprozesse auf Angebots- und Nachfrageseite werden im Vergleich mit einer Situation ohne Vertragsabschlüsse die Handelsmengen der Kassamärkte in $\mathrm{t}=0$ und $\mathrm{t}=1$ geringer sein, die Abbaumenge in $t=1$ hoher sein, der Preis $p_{0}$ höher und der Preis $p_{1} e$ geringer sein. 


\subsection{Ressourcenabbau mit Terminkontrakten}

Neben den beiden Kassamärkten in $t=0$ und $t=1$ soll nun für jeden Zeitpunkt ein Terminkontraktmarkt existieren. Den Abbauunternehmen wird damit die Möglichkeit gegeben, ihre zukünftigen Verkaufsmengen durch Hedgingtransaktionen abzusichern. Der Preis für einen morgen fälligen Terminkontrakt beträgt heute $p_{0}{ }^{f}$. Der Preis des Terminkontraktes bei Fälligkeit ist für den Abbauunternehmer eine Zufallsvariable $p_{1}{ }^{f}$, über die er sich Erwartungen $p_{1}$ fe bildet. Die Varianz des Terminkontraktpreises sei $\sigma_{\mathrm{pf}}{ }^{2}$.

Nimmt der Abbauunternehmer eine Hedgingposition ein, dann verkauft er einen Teil $\alpha(0 \leq \alpha \leq 1)$ seiner zukünftigen Abbaumengen $q_{1}$ zum heute herrschenden Terminkontraktpreis $p_{0} f$. Vor Fälligkeit des Terminkontraktes tätigt er für die Menge $\alpha q_{1}$ das Gegengeschäft zum Preis $p_{1} f$ und bietet die Menge $q_{1}$ am Kassamarkt in $t=1$ zu dem Preis $p_{1}$ an.

Der Gewinn eines Abbauunternehmers in einer Hedgingposition wird unter diesen Annahmen zu

$$
\mathbf{G}=(1+\delta) p_{0} q_{0}+\left(p_{0}^{f}-p_{1}^{f}\right) \alpha q_{1}+p_{1} q_{1} .
$$

$\left(p_{0} f-p_{1} f\right) \alpha q_{1}$ steht für den Erlös aus der Hedgingposition.

Für den Fälligkeitszeitpunkt des Terminkontraktes kann angenommen werden, daß der Terminkontraktpreis und der Kassamarktpreis für $t=1$ zusammenfallen oder eine positive Differenz aufweisen. Die Differenz zwischen diesen Preisen ist als "Basis" $b_{1}$ definiert:1) $b_{1}=p_{1}{ }^{f}-p_{1}$. Ihr Erwartungswert ist $b_{1} e=p_{1}$ fe $-p_{1} e$ und die Varianz sei $\sigma b^{2}$.

1) Vgl. hierzu Peck (1975). Es ist ein empirisches Ergebnis, daß die Basis einen stabileren zeitlichen Verlauf aufweist als die ihr zugrundeliegenden Zeitreihen für $p_{1} f$ und $p_{1}$. 
In Termini der Basıs lautet der Gewınn

$\left(5.31^{\prime}\right) \quad \mathbf{G}=(1+\delta) p_{0} q_{0}+\left[\alpha\left(p_{0} f-b_{1}\right)+(1-\alpha) p_{1}\right] q_{1}$.

Aus dem Optımierungskalkül

(5.32) $\max \quad E[U(G)]$

$\left(q_{0}, q_{1}, \alpha\right)$

u.d.B. $\quad R_{0} \geq q_{0}+q_{1}, R_{0}$ gegeben, $q_{0}, q_{1}, a \geq 0$

ergeben sich zur Bestimmung des Angebotsverhaltens die notwendigen Bedingungen:

(5.33)(i) $\quad E\left(U^{\prime}\right)(1+\delta) p_{0}-\lambda$

$\begin{cases}=0 & q_{0}>0 \\ & \text { für } \\ \leq 0 & q_{0}=0\end{cases}$

(5.33)(ii) $E\left\{U^{\prime}\left[\alpha\left(p_{0} f-b_{1}\right)+(1-\alpha) p_{1}\right]\right\}-\lambda \quad\left\{\begin{array}{cc}=0 & q_{1}>0 \\ & \text { für } \\ \leq 0 & q_{1}=0\end{array}\right.$

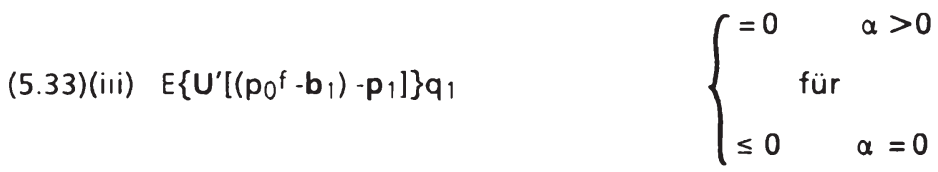

(5.33)(iv) $R_{0}-q_{0}-q_{1} \quad\left\{\begin{array}{ccc}=0 & \lambda>0 \\ & \text { für } & \\ \geq 0 & \lambda=0\end{array}\right.$ 
Positive Handelsmengen auf allen Märkten führen zu den Gleichungen

(5.34)(i) $\quad(1+\delta) p_{0}=\lambda E\left(U^{\prime}\right)-1$

(5.34)(ii) $\alpha\left\{p_{0} f-\left[b_{1} e+\operatorname{cov}\left(U^{\prime}, b_{1}\right) E\left(U^{\prime}\right)^{-1}\right]\right\}+(1-\alpha)\left[p_{1} e+\operatorname{cov}\left(U^{\prime}, p_{1}\right) E\left(U^{\prime}\right)^{-1}\right]=$ $\lambda E\left(U^{\prime}\right)-1$

(5.34)(iii) $p_{0} f=b_{1} e+p_{1} e+\left[\operatorname{cov}\left(U^{\prime}, b_{1}\right)+\operatorname{cov}\left(U^{\prime}, p_{1}\right)\right] E\left(U^{\prime}\right)^{-1}$.

In einer Hedgingposition ( $\alpha>0$ ) ist der Abbauunternehmer indifferent zwischen einem Angebot auf dem Kassamarkt in $t=0$ und $t=1$, denn es gilt

$(5.35)(i) \quad(1+\delta) p_{0}=p_{1} e+\operatorname{cov}\left(U^{\prime}, p_{1}\right) E\left(U^{\prime}\right)-1$ und

(5.35)(ii) $\left.p_{0}^{f}=p_{1} f e+\operatorname{cov}\left(U^{\prime}, p_{1}^{f}\right) E\left(U^{\prime}\right)-1.1\right)$

Der aufdiskontierte Kassamarktpreis $p_{0}$ ist gleich dem um eine Risikorate korrigierten, erwarteten Kassamarktpreis $p_{1} e$, und der heutige Terminkontraktpreis für die morgige Lieferung ist gleich dem um eıne Risikorate korrigierten, erwarteten Terminkontraktpreis morgen. Da für einen risikoaversen Abbauunternehmer $\operatorname{cov}\left(U^{\prime}, p_{1} f\right)>0$, gilt in $5.35 p_{0} f>p_{1} f e$. Eine Absicherung über den Terminkontraktmarkt liefert also einen positiven Beitrag zum optimalen Wert der Zielfunktion.

Im Erwartungsnutzenmaximum zeigt der Abbauunternehmer das folgende Angebotsverhalten:

1) Diese Schreibweıse ist äquivalent der in Gleichung 5.34 (iii). 


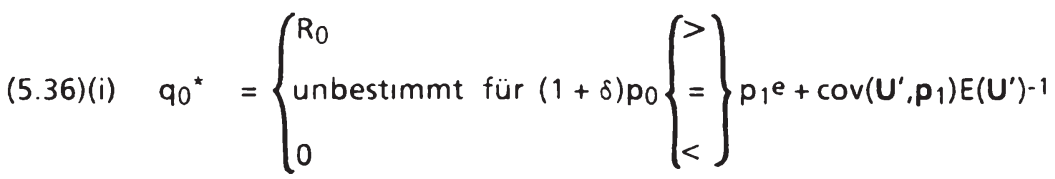

(5.36)(ii) $q_{1}^{*}=R_{0}-q_{0}^{*}$.

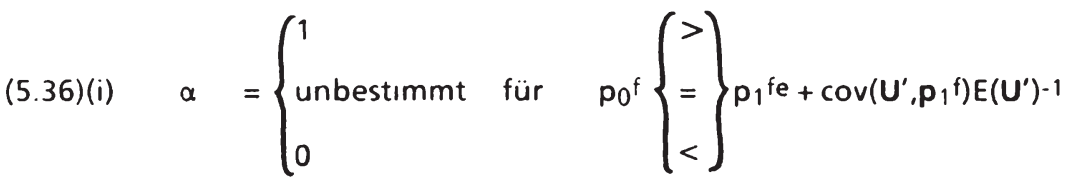

Bei Indifferenz des Abbauunternehmers wächst der erwartete Preis des Kassamarktes mit einer größeren Rate als $1+\delta:(1+\delta) p_{0}<p_{1}$ e. Ein Vergleich mit einem risikoneutralen Abbauunternehmer liefert auch hier das Ergebnis, daß der risikoaverse Abbauunternehmer trotz Hedging unterkonservierend abbaut.

Mit dem portfoliotheoretischen Instrumentarium läßt sich die folgende Aussage ableiten:

Die intertemporale Ressourcenallokation variiert mit dem Anteil der über den Terminkontraktmarkt abgesicherten Angebotsmengen für den zukünftigen Kassamarkt. Je größer der Anteil $\alpha$, desto geringer fällt der Allokationsbias zugunsten der Gegenwart aus.

Unterstellt man Konvergenz für $\mathbf{p}_{1} f$ und $\mathbf{p}_{1}$ in $t=1$, so wird $b_{1}=0$.

Ein risikoaverser Abbauunternehmer kann nun für einen gegebenen optimalen Wert seines Erwartungsnutzens die Varianz seines Gewinnes auf Null reduzieren, wenn er $\alpha=1$ wählt, $d$.h. wenn er sein gesamtes für $t=1$ geplantes Angebot über den Verkauf von Terminkontrakten absichert. Unter diesen Annahmen folgt aus den notwendigen Bedingungen 5.33 (i) und (ii) die Gleichheit 1)

1) Für den Fall $0<\alpha<1$ folgt unter der Annahme $b_{1}=0$ : $(1+\delta) p_{0}=p_{0}^{f}=p_{1} e+\operatorname{cov}\left(U^{\prime}, p_{1}\right) E\left(U^{\prime}\right)^{-1}$. 


$$
(1+\delta) p_{0}=p_{0}{ }^{f}
$$

Der Terminkontraktpreis $p_{0}$ f übernimmt die Funktion eines Preises, wie er sich auf einem Terminmarkt bei Sicherheit einstellen würde. Der Preis des Rohstoffes wächst mit der Rate $1+\delta$ im Zeitraum $t=0$ bis $t=1$. Die Allokation der Ressource bzw. das Abbauprofil haben damit das Aussehen wie unter Sicherheit.

Zur portfoliotheoretischen graphischen Darstellung seien die Gewinnsituationen wieder in Termini des Erwartungswertes und der Varianz des Gewinnes beschrieben. Neben den Gewinnsituationen in 5.7 kommen hier jene dazu, bei denen eine Hedgingposition $(\alpha>0)$ eingenommen wird:

$$
E(G)=(1+\delta) p_{0} q_{0}+\left[\alpha\left(p_{0} f-b_{1} e\right)+(1-\alpha) p_{1} e\right] q_{1}
$$

$$
\left.\operatorname{var}(\mathbf{G})=q_{1}{ }^{2}\left[\alpha^{2} \sigma_{b}{ }^{2}+(1-\alpha)^{2} \sigma_{p^{2}}+2 \alpha(1-\alpha) \sigma_{b p}\right] 1\right) \text {. }
$$

Die Budgetgerade ermittelt sich hieraus als

$$
\begin{aligned}
& E(G)=(1+\delta) p_{0} q_{0}+\left[\alpha\left(p_{0} f-b_{1} e\right)+(1-\alpha) p_{1} e\right] q_{1} \Sigma^{-1} / \operatorname{var}(G), \\
& \text { wobei } \Sigma=\alpha^{2} \sigma b^{2}+(1-\alpha)^{2} \sigma_{p^{2}}-2 \alpha(1-\alpha) \sigma_{b p} .
\end{aligned}
$$

In Schaubild 5.4 wird für gegebene Preise, Preiserwartungen, Varianzen und für ein gegebenes $\alpha$ die Ressourcenallokation zwischen $t=0$ und $t=1$ bestimmt.

Während das Optimierungskalkül die Allokationsentscheidung einschließlich der Wahl des optimalen a simultan trifft, wird hier zu verschiedenen Werten von $\alpha$ die Ressourcenallokation ermittelt.

1) $\sigma_{b p}$ bezeichnet die Kovarıanz zwischen $b_{1}$ und $p_{1}$ 
Im Uhrzeigersinn enthält das Schaubild 5.4 Im rechten oberen Quadranten die Gegenüberstellung einer Indifferenzkurve mit der Budgetgeraden (Gleichung 5.38), darunter die Risikogerade $\sqrt{\operatorname{var}(G)}=\left(R_{0}-q_{0}\right) \sqrt{\Sigma}$ und daneben die Ressourcenbestandsrestriktion $R_{0}=q_{0}+q_{1}$. Die intertemporal optimale Ressourcenallokation bestımmt sich wieder als Tangentiallosung im rechten oberen Quadranten.

Schaubild 5.4: Die Allokationsentscheidung mit Termınkontrakten

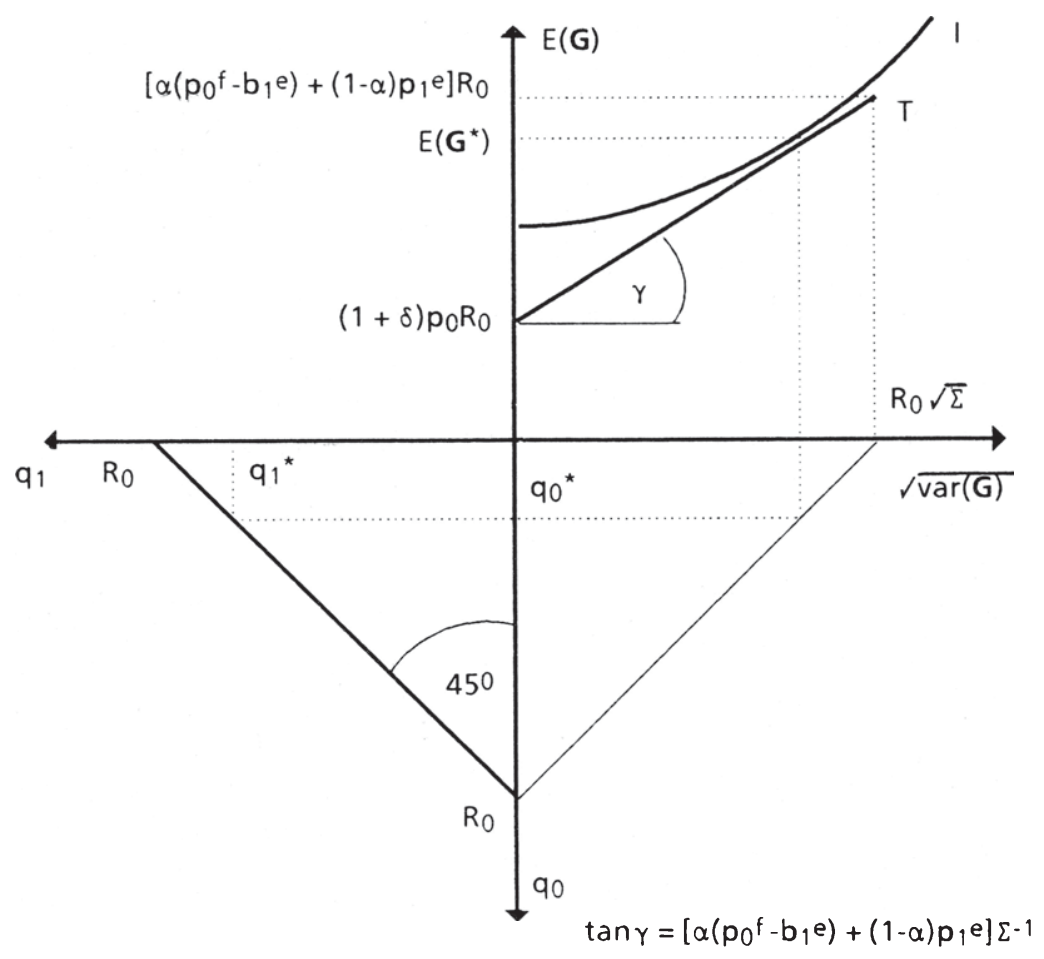


Auch hier kann der Einfluß unterschiedlicher Parametervariaționen direkt eingesehen werden:1)

Mit steigendem Risiko $\Sigma$, steigender Basis $b_{1} e$ und sinkenden Preiserwartungen $p_{1} e$ wird der Abbauunternehmer seinen Ressourcenabbau zugunsten der Gegenwart umverteilen. Die Reaktion des Abbauunternehmers auf eine Variation des Anteils $\alpha$ seiner Terminkontraktmarkttransaktionen am Rohstoffmarkt $q_{1}$ wird in Schaubild 5.5 abgeleitet. Dort sind für die beiden Grenzwerte von $\alpha, \alpha=0$ und $\alpha=1$, jeweils die Budgetgerade und die Risikogerade dargestellt.

Man erhält:

$$
\text { für } \begin{aligned}
\alpha=0: E(G) & =(1+\delta) p_{0} q_{0}+p_{1} e_{1} q_{1} \sigma_{p^{-1}} \sqrt{\operatorname{var}(G)} \\
& \operatorname{var}(G)=\left(R_{0}-q_{0}\right)^{2} \sigma_{p^{2}}
\end{aligned}
$$

(5.37') für $\alpha=1: E(G)=(1+\delta) p_{0} q_{0}+\left(p_{0} f-b_{1} e\right) q_{1} \sigma_{b^{-1}} \sqrt{\operatorname{văr}(G)}$

$$
\operatorname{var}(G)=\left(R_{0}-q_{0}\right)^{2} \sigma_{b}{ }^{2} .
$$

Es gelte die Annahme, $\sigma_{p}^{2}>\sigma b^{2}$.

Hieraus folgt für die Steigung der Budgetgeraden:

$$
\begin{aligned}
& p_{1} e \sigma_{p^{-1}}><\left(p_{0} f-b_{1} e\right) \sigma_{b}-1 \Leftrightarrow \sigma_{b} p_{1} e><\left(p_{0} f-b_{1} e\right) \sigma_{p} \\
& \Leftrightarrow \quad 0><\left(p_{0} f-p_{1} f e\right) \sigma_{p}+p_{1} e\left(\sigma_{p}-\sigma_{b}\right) .
\end{aligned}
$$

1) Nicht zu ermitteln ist hier der Einfluß der Parametervarıation auf den Anteil $\alpha$, da die Terminkontrakttransaktion des Hedging als risikobehaftet in das Modell eingeht und nicht von dem risikobehafteten Angebot für den zukünftigen Kassamarkt getrennt werden kann. 
Wegen $p_{0}^{f}>p_{1}$ fe (Gleichung 5.35) und $\sigma_{p}>\sigma_{b}$ folgt:

$$
p_{1} e \sigma_{p}-1<\left(p_{0} f-b{ }_{1} e\right) \sigma_{b^{-1}} .
$$

Für die Risikogeraden folgert man direkt aus der Annahme:

$$
\operatorname{var}(\mathbf{G})_{\alpha=0}>\operatorname{var}(\mathbf{G})_{\alpha=1} .
$$

Je größer der Anteil der über den Terminkontraktmarkt abgesicherten Abbaumengen $q_{1}$, desto mehr plant der Abbauunternehmer in $t=1$ anzubieten.

Wie sehen hier die Gleichgewichte auf den Märkten aus?

Die Gleichgewichtssituationen auf den beiden Kassamärkten lassen sich so beschreiben, wie es in den Gleichungen 5.9 bis 5.15 geschehen ist. Hinzu kommt die Beschreibung der Gleichgewichte auf den beiden Terminkontraktmärkten für $\mathrm{t}=0$ und $\mathrm{t}=1$.

Als Anbieter auf dem Terminkontraktmarkt in $t=0$ treten neben den $A b$ bauunternehmen (long hedging), $q_{0} f_{a}$, auch Wirtschaftssubjekte auf, die short hedging betreiben oder spekulieren, a 0 .

Das Angebot der Abbauunternehmer ist

$$
q_{0} f^{f a}=\Sigma_{1=1 N} \alpha_{i} q_{1 i} .
$$

In Höhe dieses Angebots treten die Abbauunternehmer dann auch als Nachfrager auf dem Terminkontraktmarkt in $t=1$ auf, $q_{1}$ fn $=q_{0}$ fa. Daneben können auch hier wieder Spekulanten und Short-Hedger als Nachfrager auftreten, $n_{1}$.

Die Nachfrage nach Terminkontrakten in $\mathrm{t}=0$ und das Angebot an Terminkontrakten in $t=1$ sei unterstellt als

$$
p_{0}^{f}=F_{0}\left(q_{0}^{f n}\right)
$$


Schaubild 5.5: Die Allokationsentscheidung in Abhängıgkeıt vom Terminkontraktanteil

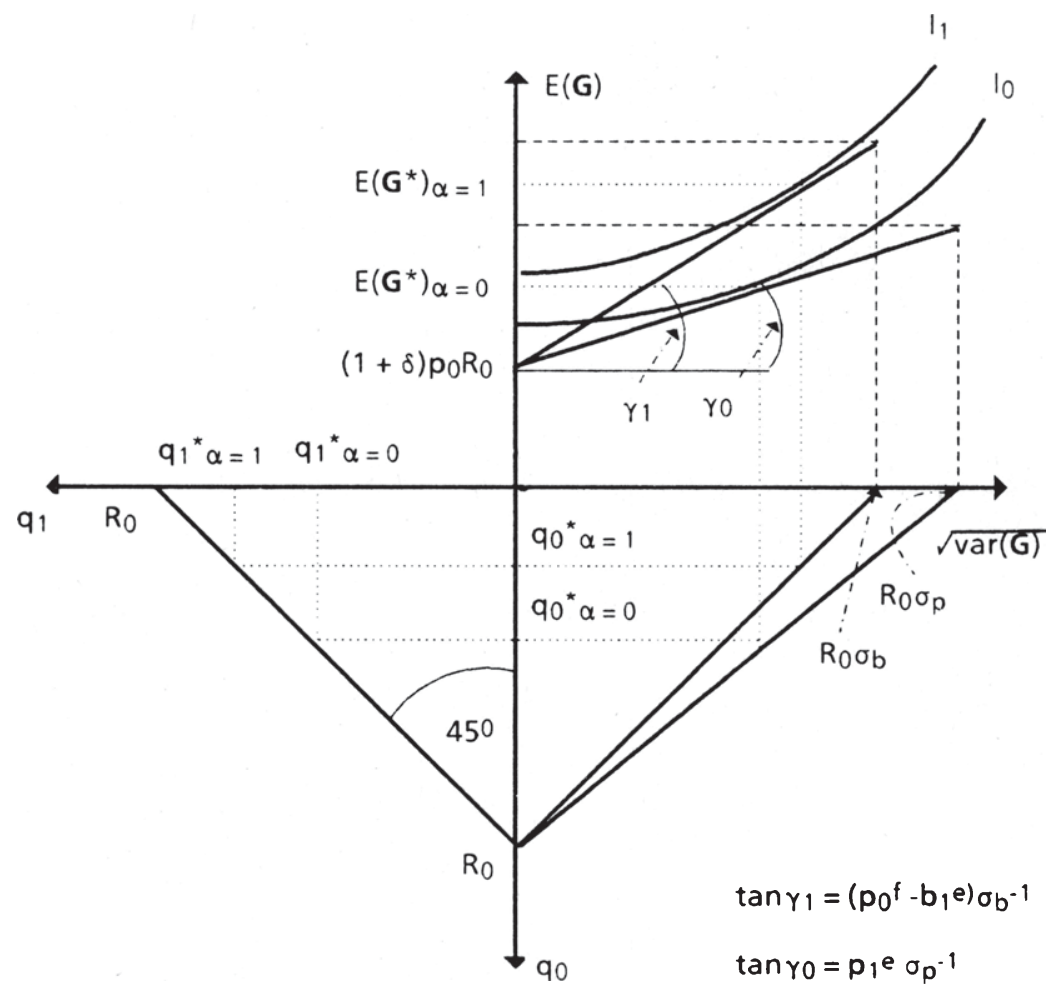

(5.41) $\quad \mathbf{p}_{1}^{f}=F_{1}\left(q_{1} f a\right) u_{1}^{f} \operatorname{mit} E\left(u_{1} f\right)=1$.

Seien die Abbaumengen $q_{01}, q_{11}$ und die Anteile $\alpha_{1}$ fur gegebene Presse bzw. Preiserwartungen Maximierer eines Optımierungskalküls der Art 5.32 und besitzen die Abbauunternehmer rationale Erwartungen auch hinsıchtlich des Terminkontraktpresses in $t=1, d . h . p_{1} f e=F_{1}(q, f n)$, dann herrscht 
Gleichgewicht auf den Termınkontraktmärkten,1) wenn gilt:

(5.42) $q_{0}{ }^{f n}=q_{0} f a+a_{0}$ und $q_{1}{ }^{f a}=q_{0} f^{f a}+n_{0}$.

Zwischen den Preısen gelten ım Gleıchgewicht die Bezıehungen:

$(543)(1) \quad(1+\delta) p_{0}=p_{1} e+\operatorname{cov}\left(U^{\prime}, p_{1}\right) E\left(U^{\prime}\right)^{-1}$ und

(5.43)(ii) $\quad p_{0}^{f}=b_{1} e+p_{1} e+\left[\operatorname{cov}\left(U^{\prime}, b_{1}\right)+\operatorname{cov}\left(U^{\prime}, p_{1}\right)\right] E\left(U^{\prime}\right)-1$.

1) Gleıchzeitıg gelten die Gleıchgewichtsbedingungen auf den Kassamarkten. 


\subsection{Ressourcenabbau mit Optionskontrakten}

Auf Optionsmärkten handeln Wirtschaftssubjekte Kauf- und Verkaufoptionen. Dem Käufer einer Kauf- oder Verkaufoption steht die Móglichkeit dann offen, den Kauf oder Verkauf spater zu dem vereinbarten Preis, dem "striking price", zu tätigen oder nıcht. ") Der Verkaufer einer Kauf- oder Verkaufoption dagegen steht in der Pflicht, die Entscheidung des Käufers zu akzeptieren.

Es sei wieder ein repräsentativer Abbauunternehmer betrachtet, der seınen bekannten Ressourcenbestand $R_{0}$ über zwei Perioden abzubauen plant. Um sich gegen einen Preisverfall auf dem zukünftigen Kassamarkt abzusichern, kauft er für die in $t=1$ abzubauende Rohstoffmenge q, Optionskontrakte (Verkaufoption) zum Preis von $p_{c}$ je Rohstoffeinheit. Der in diesen Kontrakten vereinbarte Rohstoffpreis sei mit $p_{1}$ s bezeichnet.

Als Gewinn ergibt sich für den so handelnden Abbauunternehmer

$$
\mathbf{G}=(1+\delta) p_{0} q_{0}-(1+\delta) p_{c} q_{1}+\gamma p_{1} s q_{1}+(1-\gamma) p_{1} q_{1} .
$$

Hierin ist $y$ ein Parameter, der gleich Null ist, wenn der Abbauunternehmer die Option verfallen läßt, und der gleich Eins zu setzen ist, wenn die Verkaufoption zum "striking price" $p_{1} s$ wahrgenommen wird.

Das Optimierungskalkül

$$
\begin{aligned}
& \max _{\left(q_{0}, q_{1}\right)} E[U(G)] \\
& \text { u.d.B. } \quad R_{0} \geq q_{0}+q_{1}, R_{0} \text { gegeben, } q_{0}, q_{1} \geq 0
\end{aligned}
$$

1) Im Falle eıner Kauf- (Verkauf-) optıon ıst der "strıkıng prıce" für den Käufer der Option als ein Hochstpreis (Mindestpreis) aufzufassen. 
führt zu den notwendigen Bedıngungen:

(5.46)(i) $\quad E\left(U^{\prime}\right)(1+\delta) p_{0}-\lambda$

$\begin{cases}=0 & q_{0}>0 \\ & \text { für } \\ \leq 0 & 9_{0}=0\end{cases}$

(5.46)(ii) $E\left\{U^{\prime}\left[-(1+\delta) p_{c}+\gamma p_{1} s+(1-\gamma) p_{1}\right]\right\}-\lambda \begin{cases}=0 & q_{1}>0 \\ & \text { für } \\ \leq 0 & q_{1}=0\end{cases}$

(5.46)(iii) $\left.E\left[U^{\prime}\left(p_{1} s-p_{1}\right) q_{1}\right] 1\right)$

$$
\leq 0 \text { für } \gamma=0
$$

$\left\{\begin{array}{lll}=0 & & \lambda>0 \\ & \text { für } & \\ & & \lambda=0 .\end{array}\right.$

(5.46)(iv) $R_{0}-q_{0}-q_{1}$

Wenn der Abbauunternehmer im Erwartungsnutzenmaximum positive Angebotsmengen für beide Perioden plant, dann muß gelten

(5.47)(i) $\quad(1+\delta) p_{0}=\lambda E\left(U^{\prime}\right)^{-1}$ und

(5.47)(ii) $\quad \gamma p_{1} s-(1+\delta) p_{c}+(1-\gamma)\left[p_{1} e+\operatorname{cov}\left(U^{\prime}, p_{1}\right) E\left(U^{\prime}\right)^{-1}\right]=\lambda E\left(U^{\prime}\right)^{-1}$.

Nach 5.46 (iii) ist es für den Abbauunternehmer optimal, $\gamma=0$ zu wählen, wenn die Relation

$$
p_{1} s \leq p_{1} e+\operatorname{cov}\left(U^{\prime}, p_{1}\right) E\left(U^{\prime}\right)-1 \text { gilt. }
$$

Andernfalls hat er $\gamma=1$ zu wählen, d.h. die Verkaufoption zu nutzen.

1) Dies ist die Ableıtung der Lagrangefunktion an der Stelle $\gamma=0$. 
Je nachdem, ob $\gamma=0$ oder $\gamma=1$ Ist, folgen für die Angebotsındifferenz des Abbauunternehmers die Bedingungen:

(5.49)(i) $\quad(1+\delta) p_{0}=p_{1} e+\operatorname{cov}\left(U^{\prime}, p_{1}\right) E\left(U^{\prime}\right)^{-1}-(1+\delta) p_{c}$ für $\gamma=0$

(5.49)(ii) $(1+\delta) p_{0}=p_{1} s-(1+\delta) p_{c} \quad$ fur $\gamma=1$.

Da $p_{1} s$ als ein Verkaufspreis zu interpretıeren ist, den der Abbauunternehmer mindestens erhàlt, ergibt sıch die Relation

$$
(1+\delta) p_{0}<p_{1} s<p_{1} e .
$$

Nimmt der Abbauunternehmer die Verkaufoption wahr, so realisiert er eine Steigerung des Rohstoffpreises von mehr als $1+\delta$. Läßt der Abbauunternehmer die Verkaufoption verfallen wegen (Gleichung 5.48) $p_{1} e>$ $p_{1}$ s, so realisiert er eine Steigerung des Rohstoffpreises, die die des Preises $p_{1}$ s noch übersteigt.

Für die intertemporale Ressourcenallokation folgert man, daß bei Existenz von Optionskontrakten der risikoaverse Abbauunternehmer die Ressource im Vergleich mit einer Situation ohne Optionskontrakte dann stärker unterkonserviert, wenn er die Verkaufoption verfallen läßt. Das Maß der Unterkonservierung nimmt ab, wenn der Abbauunternehmer die Verkaufoption wahrnimmt.

In den Perioden $\mathrm{t}=0$ und $\mathrm{t}=1$ bietet der $\mathrm{Abbau}$ unternehmer an gemä $\beta$

(5.50)(i) $q_{0}^{*}=\left\{\begin{array}{ll}R_{0} \\ \text { unbestimmt für }(1+\delta) p_{0} \\ 0\end{array}=A\right.$ mit $A=\gamma p_{1} s-(1+\delta) p_{c}+(1-\gamma)\left[p_{1} e+\operatorname{cov}\left(U^{\prime}, p_{1}\right) E\left(U^{\prime}\right)-1\right]$

(5.50)(ii) $q_{1}^{*}=R_{0}-q_{0}^{*}$ 
(5.50)(iii) $\gamma=\left\{\begin{array}{lll}1 & \text { für } p_{1} s & \\ & \\ 0\end{array}\right\} p_{1} e+\operatorname{cov}\left(U^{\prime}, p_{1}\right) E\left(U^{\prime}\right)-1$

Die portfoliotheoretısche Betrachtungswerse zeigt auch hier wieder das Ergebnis, daß der risıkoaverse Abbauunternehmer die Wahl hat, einerseits kleıne Gewınnerwartungen mit kleınen Gewınnvarianzen und andererseits große Gewinnerwartungen mit großen Gewinnvarianzen zu realisieren. Optıonsmärkte verschaffen dem Abbauunternehmer, ebenso wie Terminkontraktmäkte oder Lieferverträge die Möglichkeit, mit Erwartungswerten und Varianzen des Gewinnes zu "handeln", d.h. eine große Varianz des Gewinnes gegen kleinere bzw. Nullvarianz einzutauschen um den "Preis" verminderter Gewinnerwartungen:

$$
\begin{aligned}
E(G)_{Y}=0 & =(1+\delta) p_{0} q_{0}+\left[p_{1} e-(1+\delta) p_{C}\right] q_{1} \\
\operatorname{var}(G)_{Y}=0 & =q_{1}{ }^{2} \sigma p^{2}
\end{aligned}
$$

$$
\begin{aligned}
E(G)_{Y} & =1=(1+\delta) p_{0} q_{0}+\left[p_{1} s-(1+\delta) p_{C}\right] q_{1} \\
\operatorname{var}(G)_{Y}=1 & =0 .
\end{aligned}
$$

Wegen $p_{1} e>p_{1}$ s gilt $E(G)_{\gamma}=0>E(G)_{\gamma}=1$.

Für die Beschreibung der Gleichgewichte auf den drei Märkten soll für die Kassamarktnachfrage wieder von den Annahmen ausgegangen werden, wie sie in Abschnitt 5.1. eingeführt wurden:

Kassamarkt in $\mathrm{t}=0$,

(5.52) Nachfrage: $p_{0}=N_{0}\left(q_{0}^{n}\right)$ mit $N_{0}^{\prime}<0$

(5.53) Angebot: $90^{\mathrm{a}}=\sum_{-1} \mathrm{~N}_{\mathrm{N}}$ 
Kassamarkt in $\mathrm{t}=1$,

(5.54) Nachfrage: $p_{1}=N_{1}\left(q_{1} n\right) u_{1}$ mit $N_{1}{ }^{\prime}<0$ und $E\left(u_{1}\right)=0$

(5.55) Angebot: $q_{1}{ }^{a}=R_{0}-q_{0} a, R_{0}=\Sigma=, R_{0}$

Rationale Erwartungen: $p_{1} e=N_{1}(q, n)$

Das Angebot an Verkaufoptionen in $t=0$ für $t=1$ sel unterstellt als

$$
p_{c}=A_{0}\left(q_{1} 0, p_{1} s\right) \text { mit } \partial A_{0} / \partial q_{1} 0>0, \partial A_{0} / \partial p_{1} s>0
$$

Je höher der Preis für eine Verkaufoption, desto mehr Optionen werden zum Verkauf angeboten und je höher der striking price, desto hoher auch der für die Option geforderte Preis.

Da die Abbauunternehmer ihr gesamtes, geplantes Angebot q Verkaufoptionen absichern, ergibt sich für die Nachfrage auf dem Optionsmarkt $q_{1} n=R_{0}-q_{0}{ }^{a}$.

Seien die Mengen q0i und $q_{1}$ Maximierer des Optimierungskalküls ın 5.45 , dann liegt auf allen drei Märkten Gleichgewicht vor, wenn gılt:

(5.57)(i) $\quad q_{0}{ }^{a}=q_{0}^{n}$, Gleichgewicht auf dem Kassamarkt in $t=0$

(5.57)(ii) $q_{1} a=q_{1} 0$, Gleichgewicht auf dem Optionsmarkt in $t=0$

(5.57)(iii) $q_{1}^{n}=q_{1} a$, Gleichgewicht auf dem Kassamarkt in $t=1$.

Die Gleichgewichtspreise stehen zueinander in der Bezıehung, wie sie in den Gleichungen 5.49 zum Ausdruck kommen. 


\section{Die Allokationswirkungen von Vertragsvereinbarungen in Mehrperiodenmodellen des Ressourcenabbaus}

Die Analyse spezıalisiert sich nun auf die Betrachtung der allokativen Effekte der Vertragsvereınbarungen, die in Kapitel 4 und 5 unter anderen Substituten für fehlende Zukunftsmärkte erortert wurden.

Abschnitt 6.1. diskutiert Vertragsvereinbarungen des Rohstoffhandels bei verschiedenen Marktformen des Vertragshandels und unterschiedlich gestalteten Vertragsinhalten.

Abschnitt 6.2. erweitert die Betrachtungsweise um einen Reservenmarkt und diskutiert beı Nachfragerisiko auf dem Rohstoffmarkt die Allokationswirkungen von Vereinbarungen auf dem Reservenmarkt. Eingegangen wird auf die drei am häufigsten vorkommenden Vereinbarungsarten der Konzession, der Abbaumengenteilung und des Dienstleistungsvertrages. Die beiden letzten zählen dabei zu den neueren Vertragsformen.

\subsection{Vertragsvereinbarungen auf dem Rohstoffmarkt}

In den Modellansätzen zur Ressourcenallokation unter Risiko (Kapitel3) blieb unberücksichtigt, daß zwischen Ressourcenanbietern und Ressourcennachfragern langfristige Lieferverträge als institutionalisierte Substitute für fehlende Zukunftsmärkte möglich sind. Roberts (1980) führt diese Art der Reduktion von Risiko in seine Modellbetrachtung ein. Er analysiert die Auswirkung von langfristigen Lieferverträgen auf das Abbauverhalten, ohne jedoch die in der ressourcentheoretischen Analyse übliche Bestandsrestriktion für die Ressource zu berücksichtigen. Das intertemporale Angebotsverhalten des Ressourcenbesitzers erhält Roberts aufgrund einer vom Ressourcenbestand abhängigen Funktion für die Abbaukosten. 
Hier sollen die Allokationswirkungen von langfristigen Verträgen im ressourcentheoretischen Modellrahmen ohne eine bestandsabhängıge Kostenfunktion des Abbaus aber mit Bestandsrestriktion diskutiert werden.

Lieferverträge sind recht komplizierte Gebilde. Für die Modellbildung muß daher von vielen Modalitäten abgesehen werden, zum Beispiel von detaillierten Liefer- und Zahlungsbedingungen, Handhabung von Qualitätsunterschieden, Eigentumsübertragungen, Garantieverpflichtungen, etc.

In den modellmäßig betrachteten Lieferverträgen werden festgeschrieben: der für jede Periode geltende Preis, die zu handelnde Periodenmenge und der Lieferzeitraum.

Hinsichtlich dieser drei Vertragselemente sind nun verschiedene Ausgestaltungen möglich:

(1) Preise und Mengen sind im Vertragszeitraum konstant.

(2) Preise und Mengen verändern sich mit gegebener Rate.

(3) Die Laufzeit der Verträge wird als Datum vorgegeben oder wird im Kalkül bestimmt, d.h. der Abbauunternehmer bestimmt seine optimale Vertragsdauer.

(4) Die Preise sind als Daten vorgegeben oder werden als abhängig betrachtet von den Periodenmengen und/oder dem Lieferzeitraum.

(5) Die Vertragsdauer kann für alle Verträge als gleich betrachtet oder als verschieden zugelassen werden. Alle Verträge beginnen mit Aufnahme der Abbautätigkeit.

Ausgehend von dem Nach frageverhalten eines rohstoffverarbeitenden Unternehmens werden die Bestimmungsfaktoren für den Abschluß von Lieferverträgen diskutiert. Dabei wird für das Angebotsverhalten eines Abbauunternehmens auf die Ergebnısse des Abschnittes 3.1.1. zurúckgegriffen. 
Die Analyse der Allokationswırkungen von Lieferverträgen wird anschliessend durchgefuhrt.

6.1.1. Bestimmungsfaktoren für den Abschluß von Lieferverträgen unter Berücksichtiqung des Nachfrageverhaltens eines rohstoffverarbeitenden Unternehmens

Zunächst ist das Nachfrageverhalten eines rohstoffverarbeitenden Unternehmens bei Faktorpreisunsicherheit zu bestimmen.

Ein Verarbeitungsunternehmen produziere unter Einsatz der Rohstoffmenge $q(t)$ mit der Produktionsfunktion $y(t)=f(q(t)), d f / d q=f^{\prime}>0$ und $d^{2} f / d q^{2}=f^{\prime \prime}<0$, das Gut $y(t)$. Dieses kann zum Preis $p_{2}(t)$ unter vollkommener Konkurrenz abgesetzt werden. Für das Fertigprodukt existiere kein Preisrisiko.

Unbekannt ist dem Unternehmer im Planungszeitpunkt der Faktorpreis des Rohstoffes $p_{k}(t)$. 1) Zukunfstmärkte existieren nicht. Der Rohstoffpreis ist eine Zufallsvariable. Auch auf dem Rohstoffmarkt herrsche vollkommene Konkurrenz.

Der Verarbeitungsunternehmer besitze eine Nutzenfunktion $U_{2}\left(G_{2}\right)$ mit den bekannten Eigenschaften $U_{2}^{\prime}>0, U^{\prime \prime}{ }_{2}<0$ be Risikoaversion, $U^{\prime \prime}{ }_{2}>0$ bei Risikofreude. Maximiert wird der Erwartungsnutzen über dem Gewinn

$$
G_{2}={ }_{0} \int^{\top}\left[p_{2}(t) f(q(t))-p_{k}(t) q(t)\right] e^{-\delta t d t}
$$

1) Vgl. zu einer ànnlichen Modellstruktur im statischen Kontext bspw. Blair (1974). 
(6.1)

$$
\max _{(q(t))} E\left[U_{2}\left(G_{2}\right)\right] \quad \text { u.d.B. } q(t) \geq 0 .
$$

Als notwendige Bedingung wird erhalten:

$$
E\left\{U_{2}^{\prime}\left[p_{2}(t) f^{\prime}(q(t))-p_{k}(t)\right]\right\} e^{-\delta t} \quad \begin{cases}=0 & q(t)>0 \\ & \text { für } \\ \leq 0 & q(t)=0 .\end{cases}
$$

Eine positive Faktoreinsatzmenge $q(t)>0$ liefert im Optımum die Gleichung

$$
E\left[U^{\prime}{ }_{2} p_{k}(t)\right] E\left(U^{\prime}{ }_{2}\right)^{-1}=p_{2}(t) f^{\prime}(q(t)) .
$$

Setzt man ein

$$
E\left[U^{\prime}{ }_{2} p_{k}(t)\right]=E\left(U^{\prime}{ }_{2}\right) E_{2}\left[p_{k}(t)\right]+\operatorname{cov}\left[U^{\prime}{ }_{2}, p_{k}(t)\right],
$$

dann wird $6.3 \mathrm{zu}$

$$
E_{2}\left[p_{k}(t)\right]+\operatorname{cov}\left[U^{\prime}{ }_{2,} p_{k}(t)\right] E\left(U^{\prime}{ }_{2}\right)^{-1}=p_{2}(t) f^{\prime}(q(t)) .
$$

Im Gegensatz zum Ressourcenanbieter gilt fur das Risıkoverhalten des Ressourcennachfragers

$$
\operatorname{cov}\left[U_{2,}^{\prime} P_{k}(t)\right]\left\{\begin{array}{lc}
>0 & \text { Rısıkoaversion } \\
=0 & \text { bei Risıkoneutralität } \\
<0 & \text { Risikofreude. }
\end{array}\right.
$$

Man erhält das Ergebnis, daß der risıkoaverse (risikofreudige) Verarbeitungsunternehmer eine kleinere (größere) Faktornach fragemenge wahlt als jene, bei der der erwartete Faktorpreis gleich dem Wertgrenzprodukt des Rohstoffes ist. 
Definiert man sich wie in Abschnitt 3.1.1. auch hier eine Prattsche Risikopramie $Z$, so folgt

$$
E_{2}\left[p_{k}(t)\right]+d Z_{2} / d q=p_{2}(t) f^{\prime}(q(t)) .
$$

$d Z_{2} / d q$ trägt das gleiche Vorzeichen wie $\operatorname{cov}\left[U^{\prime}{ }_{2}, p_{k}(t)\right]$.

Der risikoaverse Ressourcennachfrager ist bereit, eine positive Risikoprämie über $E_{2}\left[p_{k}(t)\right]$ hinaus zu zahlen, um einen sicheren Faktorpreis zu erhalten.

Der risikofreudige Ressourcennachfrager hingegen möchte für die Zusage eines sicheren $A b n a h m e p r e i s e s ~ e i n e n ~ A b s c h l a g$ auf $E_{2}\left[p_{k}(t)\right]$ erhalten.

Wenn Ressourcenbesitzer ihren optımalen Abbauplan oder Verarbeitungsunternehmer ihren optimalen Rohstoffeinsatz bestimmen müssen, ohne die genauen zukünftigen Marktpreıse zu kennen, dann bestehen unter bestimmten Bedingungen Anreize für den Abschluß von Lieferverträgen.

Das Zustandekommen eines Liefervertrages wird (u.a.) von zwei Faktoren bestimmt: 1 )

(1) Von der subjektiven Wahrnehmung der Dichtefunktion der zukünftigen Kassapreise, bzw. den Unterschieden in der subjektiven Wahrnehmung zwischen den vertragschließenden Parteien.

(2) Von dem Risikoverhalten des Rohstoffanbieters und des Rohstoffnachfragers bzw. den Unterschieden im Risikoverhalten.

Die subjektive Wahrnehmung der Dichtefunktion zukünftiger Kassapreise bestimmt in den Kalkülen den Wert von $E\left[p_{k}(t)\right]$. Das Risikoverhalten findet seinen Ausdruck in der Änderung der Risıkopràmie $Z$ auf eine Änderung $\operatorname{der}$ Abbaumenge bzw. Faktoreinsatzmenge $q(t)$.

1) Vgl. Blair (1974), S.222. 
Es gilt

$$
Z^{\prime}=d Z / d q \begin{cases}>0 & \text { Risikoaversıon } \\ =0 & \text { bei } \\ <0 & \text { Risikoneutralität }\end{cases}
$$

des Rohstoffanbieters bzw. des Rohstoffnachfragers. $Z^{\prime}$ ist als subjektiver Risikopreis zu betrachten. 1)

Kennzeichne $E_{1}\left[p_{k}(t)\right], Z_{1}$ den Erwartungswert und die Risikoprämie eines Abbauunternehmers wie er in Abschnitt 3.1.1. diskutiert wurde, dann kann man aus den notwendigen Bedingungen der Optimierungskalküle der beiden Wirtschaftsubjekte (Gleichung 3.9' und 6.5) Kombinationen des Risikoverhaltens ableiten, unter denen Lieferverträge zustande kommen können.

$$
\begin{aligned}
& E_{1}\left[p_{k}(t)\right]=\lambda e^{\delta t E}\left(U_{1}\right)^{\prime}-1+C^{\prime}(q(t))+Z_{1}^{\prime} . \\
& E_{2}\left[p_{k}(t)\right]=p_{2}(t) f^{\prime}(q(t))-Z_{2}^{\prime} .
\end{aligned}
$$

Für den Abbauunternehmer (Verarbeitungsunternehmer) ist der subjektive Risikopreis $Z_{1}{ }^{\prime}\left(Z_{2}{ }^{\prime}\right)$ der Abschlag (Aufschlag) vom (zum ) Erwartungswert des zukünftigen Kassapreises, den er gerade noch für die Beseitigung des Risikos hinzunehmen bereit ist.2)

Ein Liefervertrag wird dann zustande kommen, wenn die kleinstmögliche Preisforderung des Anbieters [ $P F(t)$ ] aufgrund seiner Kostensituation höchstens die größtmögliche Zahlungsbereitschaft des Nachfragers [ $Z B(t)$ ] aufgrund seiner Ertragslage erreicht.

1) Diese Bezeichnungsweise benutzt Sinn (1980), S.55.

2) Vgl. Sinn (1980), S.80. 
$P F(t)$ ist zu interpretieren als eıne Preisuntergrenze, die der Anbieter zu gewahren bereit ist, um diesen Press als sicheren Absatzpreis zu erhalten.

$\mathrm{ZB}(\mathrm{t})$ ist dementsprechend $\mathrm{z} \mathrm{u}$ interpretieren als eine Preisobergrenze, die der Nachfrager zu zahlen bereit ist, um diesen Preis als sicheren Faktorpreis zu erhalten.

Definiert man

$$
\begin{aligned}
& P F(t)=\lambda e^{\delta t E\left(U_{1}{ }^{\prime}\right)-1}+C^{\prime}(q(t)) \text { und } \\
& Z B(t)=p_{2}(t) f^{\prime}(q(t))
\end{aligned}
$$

so gilt in $3.9^{\prime}$ und 6.5

$$
\begin{aligned}
& P F(t)=E_{1}\left[p_{k}(t)\right]-Z_{1}^{\prime} \\
& Z B(t)=E_{2}\left[p_{k}(t)\right]+Z_{2}^{\prime} .
\end{aligned}
$$

Daraus folgt

$$
Z B(t)-P F(t)=E_{2}\left[p_{k}(t)\right]-E_{1}\left[p_{k}(t)\right]+Z_{1}{ }^{\prime}+Z_{2}{ }^{\prime} .
$$

$\mathrm{ZB}(\mathrm{t})-\mathrm{PF}(\mathrm{t}) \geq 0$ kann als eine hinreichende Bedingung für das Zustandekommen von Lieferverträgen betrachtet werden. Ein Vertragspreis $p_{v}(t)$ mit $Z B(t) \geq p_{v}(t) \geq P F(t)$ ist für beide Parteien vorteilhaft, da er einerseits die maximale Zahlungsbereitschaft des Nachfragers nicht überschreitet und die minimale Preisforderung des Anbieters nicht unterschreitet und andererseits das Preisrisiko ausschaltet.

$$
Z B(t)-P F(t) \geq 0 \Leftrightarrow Z_{1}{ }^{\prime}+Z_{2}{ }^{\prime} \geq E_{1}\left[p_{k}(t)\right]-E_{2}\left[p_{k}(t)\right] .
$$

Gemäß obiger Bedingung entstehen Vorteile aus dem Abschluß eines Liefervertrages nur dann, wenn einem bestimmten Risikoverhalten eine bestimmte Erwartungshaltung der vertragschließenden Parteien gegenübersteht. 
Um zu weiteren Aussagen zu kommen, soll die Betrachtung zunächst für identische subjektive Wahrnehmungen über die Dichtefunktionen der zukünftigen Kassapreise erfolgen: $E_{1}\left[p_{k}(t)\right]=E_{2}\left[p_{k}(t)\right]$.

Nun gilt:

$$
Z B(t)-P F(t) \geq 0 \Leftrightarrow Z_{1}^{\prime}+Z_{2}^{\prime} \geq 0 .
$$

D.h. immer dann, wenn

(1) beide Vertragspartner risikoavers sind,

(2) ein Vertragspartner risikoavers, der andere risikoneutral ist,

(3) ein Vertragspartner risikoavers und der andere risikofreudig mit $z_{1}^{\prime} \geq-z_{2}^{\prime}>0$ bzw. $z_{2}^{\prime} \geq-z_{1}^{\prime}>0$ ist,

besteht ein Anreiz, Preise festzuschreiben, also Lieferverträge abzuschliessen.

Unter (1) ist der Rohstoffnachfrager bereit, eine positive Prämie über $E_{2}\left[p_{k}(t)\right]$ hinaus zu zahlen. Der Rohstoffanbieter ist bereit, eine positive Risikoprämie als Abschlag auf $E_{1}\left[p_{k}(t)\right]$ hinzunehmen. Wird ein Preis $p_{v}(t)$ mit $Z B(t)>p_{v}(t)>P F(t)$ ausgehandelt, so trägt jeder der Handelspartner einen Teil der Risikoprämie.

Stellt man einem risikoaversen Unternehmer einen risikoneutralen oder gar risikofreudigen Verhandlungspartner gegenüber, dann verringert sich das Intervall, in dem über den Preis verhandelt werden kann.

In (2) ist der risikoneutrale Unternehmer nicht bereit, zu seinen Ungunsten von seinem Erwartungswert abzuweichen. Der risikoaverse Unternehmer hingegen zeigt die Bereitschaft, zugunsten des anderen, vertraglich von seinem Erwartungswert abzuweichen.

Unter (3) muß der risikoaverse Unternehmer zu einer positiven Risikoprämie bereit sein, die mindestens der negativen Risikoprämie des risikofreudigen Unternehmers entspricht. Gibt man identisches Risikoverhalten vor, 
dann bestimmt das Verhältnis der erwarteten Kassapreıse das Zustandekommen von Vertragsabschlüssen.

Tabelle 6.1 ordnet einem vorgegebenen Risikoverhalten und einer vorgegebenen Differenz der Erwartungswerte das Vorzeichen der Differenz $Z B(t)-P F(t), d . h$. die Vorteilhaftigkeit eines Vertragsabschlusses $Z u$. Gilt $Z B(t)$ $-P F(t) \geq 0$, so stellt sich durch Vertragsabschluß keiner der Handelspartner schlechter.

Die letzten beiden Spalten erfassen den Fall genau entgegengesetzten $\mathrm{Ri}$ sikoverhaltens.

Tabelle 6.1: Die Vorteilhaftigkeit von Vertragsabschlüssen

\begin{tabular}{c|c|c}
$Z B(t)-P F(t)$ & $E_{1}\left[p_{k}(t)\right]>E_{2}\left[p_{k}(t)\right]$ & $E_{1}\left[p_{k}(t)\right] E_{2}\left[p_{k}(t)\right]$ \\
\hline$Z_{1}^{\prime}=Z_{2}^{\prime}>0$ & $><$ & $\geq$ \\
$Z_{1}^{\prime}=Z_{2}^{\prime}=0$ & $<$ & $><$ \\
$Z_{1}^{\prime}=Z_{2}^{\prime}<0$ & $<$ & $\geq$ \\
$\left|Z_{1}^{\prime}\right|=Z_{2}^{\prime}>0$ & $<$ & $\geq$ \\
$Z_{1}^{\prime}=\left|Z_{2}{ }^{\prime}\right|>0$ & $<$
\end{tabular}

Zusammenfassend kann festgehalten werden:

(1) Bei gleichem oder unterschiedlichem Risikoverhalten können unterschiedliche Erwartungshaltungen hinreichend für Vertragsabschlusse sein. 
(2) Bei gleicher Erwartungshaltung muß einer der beiden Handelspartner ein risikoaverses Verhalten zeıgen, damit es zu Vertragsabschlüssen kommen kann.1)

Wenn die Voraussetzungen für den Abschluß gegeben sind, stellt sıch die Frage, welche Bedingungen gelten nach den Verhandlungsprozessen für eine Gleichgewichtssituation? Zur Beantwortung dieser Frage sei von folgendem Ansatz ausgegangen:

Ein Abbbauunternehmer bestimme den Anteil $v(1 \geq v \geq 0)$ seiner Abbaumengen, die er über Lieferverträge verkaufen will, so, daß er seınen Erwartungsnutzen aus der Abbautätigkeit maximiert und sein Handelspartner ein bestimmtes Gewinniveau $G_{2}{ }^{*}$ nicht unterschreitet.

Die Lieferverträge laufen über den gesamten Zeitraum $[0, T]$. Die Lieferpreise $p_{v}$ und der Anteilswert $v$ seien im Vertragszeitraum fix.

Formal lautet die Problemstellung für die paretoeffiziente Wahl der relativen Vertragshandelsmenge $v$ $\max E\left[U_{1}\left(G_{1}\right)\right]$ u.d.B. $E\left[U_{2}\left(G_{2}\right)\right] \geq U_{2}\left(G_{2}{ }^{*}\right), v \geq 0$. (v)

Hierbei ist $\mathbf{G}_{1}$ der Gegenwartswert der Gewinne des Abbauunternehmers im Abbauzeitraum [0,T]

$$
\mathbf{G}_{1}={ }_{0} \int^{\top}\left\{\left[v p_{v}+(1-v) p_{k}(t)\right] q(t)-C(q(t))\right\} e^{-\delta t d t}
$$

und $\mathbf{G}_{2}$ der Gewinn des Verarbeitungsunternehmers im selben Zeıtraum

$$
G_{2}={ }_{0} \int^{T}\left\{p_{2}(t) f(q(t))-\left\{v p_{v}+(1-v) p_{k}(t)\right]\right\} q(t) e^{-\delta t d t} .
$$

Aus der Lagrangefunktion

$$
L=E\left[U_{1}\left(G_{1}\right)\right]+\mu\left\{E\left[U_{2}\left(G_{2}\right)\right]-U_{2}\left(G_{2}^{*}\right)\right\}
$$

1) Vgl. zu diesen Schlußfolgerungen Blaır(1974), S.223. 
folgt die notwendige Bedingung

$$
E\left[U_{1}^{\prime}\left(\partial G_{1} / \partial v\right)\right]+\mu E\left[U_{2}{ }^{\prime}\left(\partial G_{2} / \partial v\right)\right] \quad\left\{\begin{array}{cc}
=0 & v>0 \\
\text { für } & \\
\leq 0 & v=0
\end{array}\right.
$$

Es gilt

(6.12 $) \quad \partial \mathbf{G}_{1} / \partial v=-\partial \mathbf{G}_{2} / \partial v={ }_{0}{ }^{\top}\left[p_{v}-p_{k}(t)\right] q(t) e^{-} \delta t d t$,

so daß folgt

(6.12') $\quad E\left[\left(U_{1}{ }^{\prime}-\mu U_{2}{ }^{\prime}\right)\left(\partial G_{1} / \partial v\right)\right]=0$.

Fur $\partial G_{1} / \partial v$ ungleich null, folgt $U_{1}{ }^{\prime}=\mu U_{2}{ }^{\prime}$ und weiter

$\left(6,12^{\prime \prime \prime}\right) \quad E\left(U_{1}^{\prime}\right)=\mu E\left(U_{2}^{\prime}\right)$.

Ferner folgt aus $6.12^{\prime \prime} 11$

(6.12 $\left.{ }^{i v}\right) \quad E\left[U_{1}^{\prime} p_{k}(t)\right]=\mu E\left[U_{2}^{\prime} p_{k}(t)\right]$.

Zusammen mit 6.12"' ergeben sich die äquivalenten Gleichungen

$$
E\left[U_{1}^{\prime} p_{k}(t)\right] E\left(U_{1}{ }^{\prime}\right)^{-1}=E\left[U_{2} p_{k}(t)\right] E\left(U_{2}{ }^{\prime}\right)^{-1}
$$

$$
E_{1}\left[p_{k}(t)\right]+\operatorname{cov}\left[U^{\prime}{ }_{1}, p_{k}(t)\right] E\left(U^{\prime}{ }_{1}\right)^{-1}=E_{2}\left[p_{k}(t)\right]+\operatorname{cov}\left[U^{\prime}{ }_{2}, p_{k}(t)\right] E\left(U^{\prime}{ }_{2}\right)^{-1}
$$

$$
E_{1}\left[p_{k}(t)\right]-Z_{1}^{\prime}=E_{2}\left[p_{k}(t)\right]+Z_{2}^{\prime}
$$

Ist der Ahteil v des Handels über Lieferverträge am gesamten Rohstoffhandel pareitoeffizient gewählt, dann stimmen die um die subjektiven Risikopreise korrigierten Erwartungswerte von Abbauunternehmen und Verarbeitungsunternehmen überein.

1) $\quad\left[E\left(U^{\prime}{ }_{1}\right)-\mu E\left(U^{\prime}{ }_{2}\right)\right]_{0} \int^{\top} p_{v} q(t) e^{-\delta t} d t=E\left[U_{1}{ }_{0}{ }_{0}^{T} p_{k}(t) q(t) e^{-\delta t} d t\right]-$

$$
\begin{aligned}
& \mu E\left[U^{\prime}{ }_{2} \delta_{0}^{\top} p_{k}(t) q(t) e^{-\delta t} d t\right]={ }_{0} \int^{\top}\left\{E\left[U^{\prime}{ }_{1} p_{k}(t)\right]-\mu E\left[U^{\prime}{ }_{2} p_{k}(t)\right]\right\} q(t) e^{-\delta t} d t . \\
& \text { Fur } p_{v}>0, q(t)>0 \text { und mit } E\left(U^{\prime}{ }_{1}\right)=\mu E\left(U^{\prime}{ }_{2}\right) \text { gilt auch } E\left[U^{\prime}{ }_{1} p_{k}(t)\right] \\
& =\mu E\left[U^{\prime}{ }_{2} p_{k}(t)\right] \text {. }
\end{aligned}
$$


Soweit die Betrachtung der Bestimmungsfaktoren für den Abschluß von Lieferverträgen und deren Vorteilhaftigkeit.

Bevor die Allokationswirkungen der langfristigen Lieferverträge diskutiert werden können, ist das Nachfrageverhalten eines Verarbeitungsunternehmers nach Rohstoffen über Lieferverträge und über den Kassamarkt zu bestimmen. Denn die Nachfrage auf den zukünftigen Kassamärkten wird ohne Lieferverträge eine andere sein als mit Lieferverträgen.

In 6.1 wurde das Nachfrageverhalten auf den Kassamärkten ohne die Existenz von Lieferverträgen bestimmt. Dem Verarbeitungsunternehmer wird nun die Möglichkeit gegeben, Lieferverträge über seinen Planungszeitraum $[0, T]$ abzuschließen. Im Lieferzeitraum setzt sich nun der Faktoreinsatz zusammen aus der Periodenliefermenge $q_{v}$ und der über den Kassahandel erworbenen Mengen $q_{k}(t)$. Der Lieferpreis $p_{v}$ und die Liefermengen seien im Vertragszeitraum konstant. Sowohl auf den Kassamärkten als auch beim Abschluß von Lieferverträgen sei der Verarbeitungsunternehmer Mengenanpasser. Die Nachfrage nach Rohstoffmengen über Vertrags- und Kassahandel bestimmt sich aus dem Optimierungskalkül

$$
\begin{aligned}
& \max _{\left(q_{v}, q_{k}(t)\right)} E\left\{U_{2}\left\{_{0} J^{T}\left[p_{2}(t) f(q(t))-p_{v} q_{v}-p_{k}(t) q(t)\right] e^{-\delta t d t}\right\}\right\} \\
& \text { u.d.B. } \quad q_{v}, q_{k}(t) \geq 0 .
\end{aligned}
$$

An notwendigen Bedingungen werden erhalten

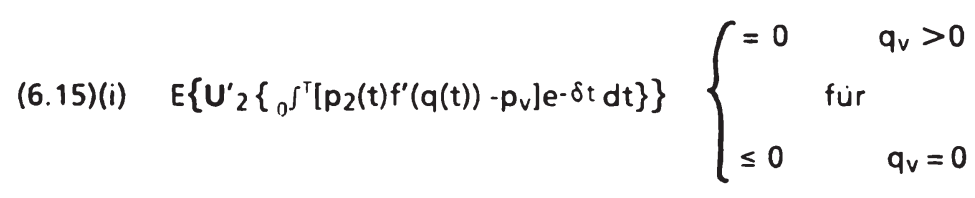


(6.15)(11) $E\left\{U^{\prime}{ }_{2}\left[p_{2}(t) f^{\prime}(q(t))-p_{k}(t)\right]\right\} e^{-\delta t} \quad \begin{cases}=0 & q_{k}(t)>0 \\ & \text { für } \\ \leq 0 & q_{k}(t)=0 .\end{cases}$

Aus beıden Gleıchungen folgt für $q_{v}, q_{k}(t)>0$

(6.16)(i) ${ }_{0} \int^{T} p_{2}(t) f^{\prime}(q(t)) e^{-\delta t} d t=p_{v}\left(1-e^{-\delta T}\right) \delta-1$

(6.16)(ii) $p_{2}(t) f^{\prime}(q(t))=E_{2}\left[p_{k}(t)\right]+\operatorname{cov}\left[U^{\prime}{ }_{2}, p_{k}(t)\right] E\left(U^{\prime}{ }_{2}\right)^{-1}$.

Im Optimum frägt der Verarbeitungsunternehmer jene Rohstoffmenge uber Vertragslieferungen nach, bei der der Barwert des Wertgrenzproduktes gleich dem Barwert des Lieferpreises über dem Lieferzeitraum ist und bei der das Wertgrenzprodukt gleich dem um den subjektiven Risikopreis nach oben korrigierten Kassapreis ist.

Die Gleichung

$$
p_{v}\left(1-e^{-\delta T}\right) \delta^{-1}={ }_{0}{ }^{T}\left\{E_{2}\left[p_{k}(t)\right]+\operatorname{cov}\left[U^{\prime}{ }_{2}, p_{k}(t)\right] E\left(U^{\prime}{ }_{2}\right)^{-1}\right\} e^{-\delta t} d t
$$

stellt in impliziter Form die Relationen der Nachfragen $q_{v}$ und $q_{k}(t)$ in Abhängigkeit von $p_{v}$ und $E_{2}\left[p_{k}(t)\right]$ dar. Man erhält für die Nachfragefunktionen

(6.18)(i) $\quad q_{v}=g_{v}\left(p_{v},\left\{E_{2}\left[p_{k}(t)\right]\right\}_{t=0} T\right)$ und

(6.18)(ii) $q_{k}(t)=g_{k}\left(E_{2}\left[p_{k}(t)\right], p_{v}\right)$

die Eigenschaften

(6.19)(i) $\partial q_{v} / \partial p_{v}=\left[A \delta\left(1-e^{-\delta T}\right)^{-1}\right]^{-1} \quad$ und $\partial q_{v} / \partial E_{2}\left(p_{k}\right)=-A-1$

(6.19)(ii) $\partial q_{k} / \partial p_{v}=\left[B \delta e^{-\delta T}\left(1-e^{-\delta T}\right)^{-1}\right]^{-1}$ und $\partial q_{k} / \partial E_{2}\left(p_{k}\right)=-B^{-1}$,

mit $\quad A={ }_{0} \int^{\top} E\left(U^{\prime}{ }_{2}\right)^{-1}\left\{d \operatorname{cov}\left[U^{\prime}{ }_{2}, p_{k}(t)\right] / d q_{v}\right\} e^{-\delta t} d t$

und $\quad B=E\left(U^{\prime}{ }_{2}\right)^{-1}\left\{d \operatorname{cov}\left[U^{\prime}{ }_{2}, p_{k}(t)\right] / d q_{k}\right\}$.

Vertragsabschlüsse mindern genau dann das Risiko, wenn gilt:

$$
d \operatorname{cov}\left[U^{\prime}{ }_{2}, p_{k}(t)\right] / d q_{v}<0 \text {. }
$$

Vermehrter Kassahandel führt zu einem größeren Risiko genau dann, wenn gilt: 


$$
\operatorname{dcov}\left[U^{\prime}{ }_{2}, p_{k}(t)\right] / d q_{k}>0 .
$$

Beide Definitionen gelten nur für einen risikoaversen Verarbeitungsunternehmer, für den Risiko definiert ist als $\operatorname{cov}\left[U^{\prime}{ }_{2}, p_{k}(t)\right]>0$.

Damit haben $A$ und $B$ eindeutige Vorzeichen, $A<0$ und $B>0$.

Die Eigenschaften der Nachfragefunktionen lauten:

(6.20)(i) $\partial q_{v} / \partial p_{v}<0$ und $\partial q_{v} / \partial E_{2}\left(p_{k}\right)>0$

(6.20)(ii) $\partial q_{k} / \partial p_{v}>0$ und $\partial q_{k} / \partial E_{2}\left(p_{k}\right)<0$.

Ein Vergleich der Eigenschaften der Nachfragefunktionen der Kassamärkte mit und ohne die Möglichkeit, langfristige Lieferverträge abzuschließen, führt zu dem Ergebnis, daß die Kassanachfrage in Gegenwart von Vertragsabschlüssen einen elastischeren Verlauf aufweist, $d$.h. in einem Preis-Mengen-Diagramm verläuft diese Nachfragefunktion flacher.

Ohne Vertragsabschlüsse gilt für die Kassanachfrage (Gleichung 6.4):

$$
d q / d E_{2}\left(p_{k}\right)=\left\{p_{2}(t) f^{\prime \prime}(q(t))-E\left(U^{\prime}{ }_{2}\right)^{-1}\left\{d \operatorname{cov}\left[U^{\prime}{ }_{2}, p_{k}(t)\right] / d q\right\}\right\}-1 ;
$$

mit Vertragsabschlüssen dagegen gilt:

$$
\partial q_{k} / \partial E_{2}\left(p_{k}\right)=\left\{-E\left(U_{2}^{\prime}\right)^{-1}\left\{d \operatorname{cov}\left[U_{2}^{\prime}, p_{k}(t)\right] / d q_{k}\right\}\right\}-1 .
$$

sodaß folgt:

$$
0>d q / d E_{2}\left(p_{k}\right)>\partial q_{k} / \partial E_{2}\left(p_{k}\right) \Leftrightarrow 0>\partial E_{2}\left(p_{k}\right) / \partial q_{k}>d E_{2}\left(p_{k}\right) / d q .
$$

Schaubild 6.1 zeigt den Verlauf der Nachfragen nach Rohstoffen über den Kassamarkt mit und ohne die Existenz von Vertragsabschlüssen. 
Schaubild 6.1: Die Nachfrage auf dem Kassamarkt für Rohstoffe mit und ohne Lieferverträge

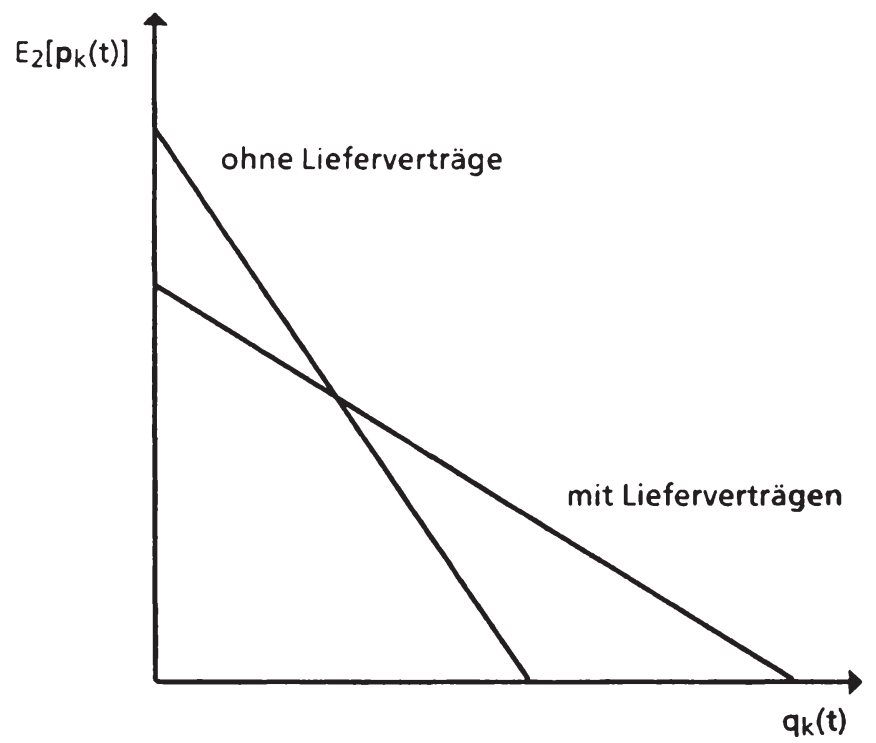




\subsubsection{Das Abbauprofil bei konkurrenzmäßig organisiertem Kassa- und Vertragshandel}

Der in Abschnitt 3.1.1. beschriebene Abbauunternehmer sei risikoavers. Er habe nun neben der Wahl des Ressourcenabbaus zwischen heute oder morgen zusätzlich die Möglichkeit, Lieferverträge für einen Zeitraum $\left[0, T_{1}\right]$ abzuschließen. Die morgen abzubauende Ressource kann heute schon verkauft werden.

Innerhalb der Laufzeit des Vertrages seien der Preis $p_{v}$ einer Liefereinhelt und die Periodenliefermenge $q_{v}$ konstant. Lieferzeitraum und Preis seien dem Abbauunternehmer als Daten vorgegeben, d.h. er steht auf den Kassamärkten und auf dem Markt für vertragliche Liefermengen (Vertragsmarkt) unter vollständiger Konkurrenz.

Die Nachfrage eines Verarbeitungsunternehmens braucht nicht durch einen Vertrag von einem einzigen Anbieter befriedigt zu werden. Ebenso braucht das Angebot eines Abbauunternehmers an vertraglichen Liefermengen nicht durch einen Vertrag von einem einzigen Nachfrager abgenommen zu werden, sondern kann sich auf mehrere Nachfrager verteilen.

Der Vertragsmarkt existiert nur im Zeitpunkt $t=0$. Bevor ein Abbau zustandekommt, ist der Vertragsmarkt geräumt. Da die Vertragsdauer vorgegeben und für alle Verträge gleich ist, braucht nicht zwischen mehreren Vertragsmärkten unterschieden zu werden.

Der repräsentative Abbauunternehmer wählt aus einer bestimmten Erwartungshaltung heraus bei gegebenem, bekanntem Ressourcenbestand $R_{0}$. $z u$ vorgegebenen Werten $p_{v}, T_{1}$ seine Liefermengen $q_{v}$ und sein Kassaangebot $q_{k}(t)$ so, daß der Erwartungsnutzen des Gewinnes aus seiner Abbautätigkeit optimal wird.

Nun ist für den Gewinn des Abbauunternehmers zu setzen

$$
\mathbf{G}={ }_{0} \int_{2}\left[p_{v} q_{v}+p_{k}(t) q_{k}(t)-C(q(t))\right] e^{-} \delta t d t .
$$

$T_{2}$ bezeichne den Zeitpunkt, in dem die Abbautätigkeit eingestellt wird. $q(t)=q_{v}+q_{k}(t)$ ist die gesamte Abbaumenge in der Periode $t$. 
Aus der Problemstellung

(6.21)

$$
\begin{array}{ll}
\max & E[U(G)] \\
\begin{array}{ll}
\left(q_{v}, q_{k}(t), T_{2}\right) & \\
\text { u.d.B. } & R_{0} \geq q_{v} T_{1}+{ }_{0} \int^{T} \geq q_{k}(t) d t, R_{0}, T_{1} \text { gegeben, } \\
& q_{k}(t) \geq 0 \text { für } t \in\left[0, T_{2}\right] \\
q_{v} \geq 0 \text { für } t \in\left[0, T_{1}\right] \\
q_{v}=0 \text { für } t \in\left(T_{1}, T_{2}\right]
\end{array}
\end{array}
$$

werden die notwendigen Bedingungen erhalten

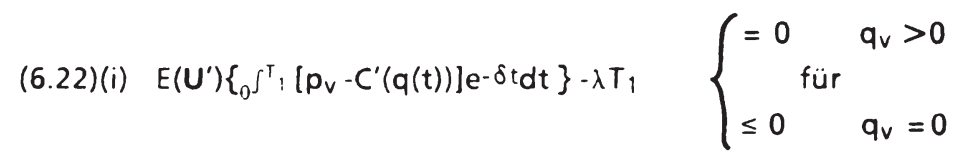

(6.22)(ii) $E\left\{U^{\prime}\left[p_{k}(t)-C^{\prime}(q(t))\right]\right\} e^{-\delta t-\lambda} \quad \begin{cases}=0 & q_{k}(t)>0 \\ & \text { für }\end{cases}$

(6.22)(iii) $R_{0}-q_{v} T_{1}-{ }_{0} \int^{T} q_{k}(t) d t$

$$
\left\{\begin{array}{lll}
=0 & \lambda>0 \\
& \text { für } & \\
\geq 0 & \lambda=0
\end{array}\right.
$$

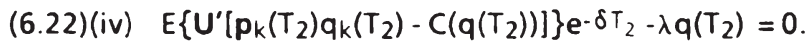

Werden im Zeitraum $\left[0, T_{1}\right]$ sowohl Verträge erfüllt, $q_{v}>0$, als auch der Kassamarkt beliefert, $q_{k}(t)>0$, so folgt aus 6.22 (i) und (ii)

(6.23)(i) $E\left(U^{\prime}\right) T_{1}-{ }_{0} \int^{T_{1}}\left[p_{v}-C^{\prime}(q(t))\right] e^{-\delta t d t}=\lambda \quad$ und (6.23)(ii) $E\left\{U^{\prime}\left[p_{k}(t)-C^{\prime}(q(t))\right]\right\} e^{-\delta t}=\lambda$. 
Die optimale Handelsmenge über Liefervertráge wird erreicht, wenn der über den Vertragszeitraum gebildete Mittelwert der Erwartungsnutzen aller diskontierten Grenzgewinne des Vertragsverkaufes gleıch dem Schattenpreis der Ressource ist.

Gleich dem Schattenpreis der Ressource ist im Optimum auch der Gegenwartswert des Erwartungsnutzens der Grenzgewinne aus dem Kassaangebot. Der Gegenwartswert des Schattenpreises ist konstant. Sein laufender Wert wächst mit der Diskontrate.

Im Optimum ist der Ressourcenbesitzer indifferent zwischen dem Verkauf einer weiteren Mengeneinheit über Lieferverträge für den Zeitpunkt t oder dem Verkauf über den Kassamarkt in $t$.

Denn es gilt

$$
\begin{aligned}
& T_{1}{ }^{-1}{ }_{0} T^{T} 1\left[p_{v}-C^{\prime}(q(t))\right] e^{-\delta t} d t=
\end{aligned}
$$

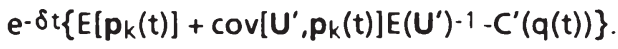

Einem geplanten Angebotsprofil für den Kassamarkt ist damit ein bestimmtes Abbauprofil und eine bestimmte Periodenliefermenge zugeordnet.

Gleichung 6.24 ist äquivalent dem Ergebnis einer weiteren Umformung1):

$$
p_{v}\left(1-e^{-\delta} T_{1}\right) \delta-1={ }_{0} \int^{T}{ }_{1}\left\{E\left[p_{k}(t)\right]+\operatorname{cov}\left[U^{\prime}, p_{k}(t)\right] E\left(U^{\prime}\right)-1\right\} e^{-\delta t d t} .
$$

1) Aus 6.23 (i) folgt

${ }_{0} \int^{T}, p_{v} e^{-\delta t} d t=\lambda T_{1} E\left(U^{\prime}\right)-1+{ }_{0} \int^{T_{1}} C^{\prime}(q(t)) e^{-\delta t} d t$.

Dies ergibt mit

$C(q(t)) e^{-\delta t}=E\left[U^{\prime} p_{k}(t)\right] E\left(U^{\prime}\right)^{-1} e^{-\delta t}-\lambda E\left(U^{\prime}\right)^{-1}$ aus 6.23 (ii) die Gleichung ${ }_{0} \int^{T_{1}} p_{v} e^{-\delta t} d t=\lambda T_{1} E\left(U^{\prime}\right)^{-1}+{ }_{0} \int^{T_{i}}\left\{E\left[U^{\prime} p_{k}(t)\right] E\left(U^{\prime}\right)^{-1} e^{-\delta t}-\lambda E\left(U^{\prime}\right)^{-1}\right\} e^{-\delta t} d t$.

Da $p_{v}$ und $\lambda E\left(U^{\prime}\right)-1$ im Zeitraum $\left[0, T_{1}\right]$ konstant sind, gilt

$p_{v}\left(1-e^{\left.-\delta T_{1}\right) \delta-1}={ }_{0} \int^{T} 1\left\{E\left[p_{k}(t)\right]+\operatorname{cov}\left[U^{\prime}, p_{k}(t)\right] E\left(U^{\prime}\right)^{-1}\right\} e^{-\delta t d t}\right.$.

Berücksichtigt wurde dabei die Definition von $\operatorname{cov}\left[\mathrm{U}^{\prime}, \mathrm{p}_{k}(\mathrm{t})\right]$. 
Der Gegenwartswert des Lieferpreises über den Vertragszeitraum ist im Optimum gleich dem Gegenwartswert der um einen Risikoabschlag verminderten Erwartungswerte für die Preise der Kassamärkte in diesem Zeitraum.

Zeichnet man beide Funktionen in Abhängigkeıt von der Periodenliefermenge $q_{v}$ in ein Schaubild ein, dann läßt sich die optimale Liefermenge ermitteln.1)

Schaubild 6.2: Die optimale vertragliche Liefermenge bei vorgegebener Vertragsdauer

Erlös in Gegenwartswerten

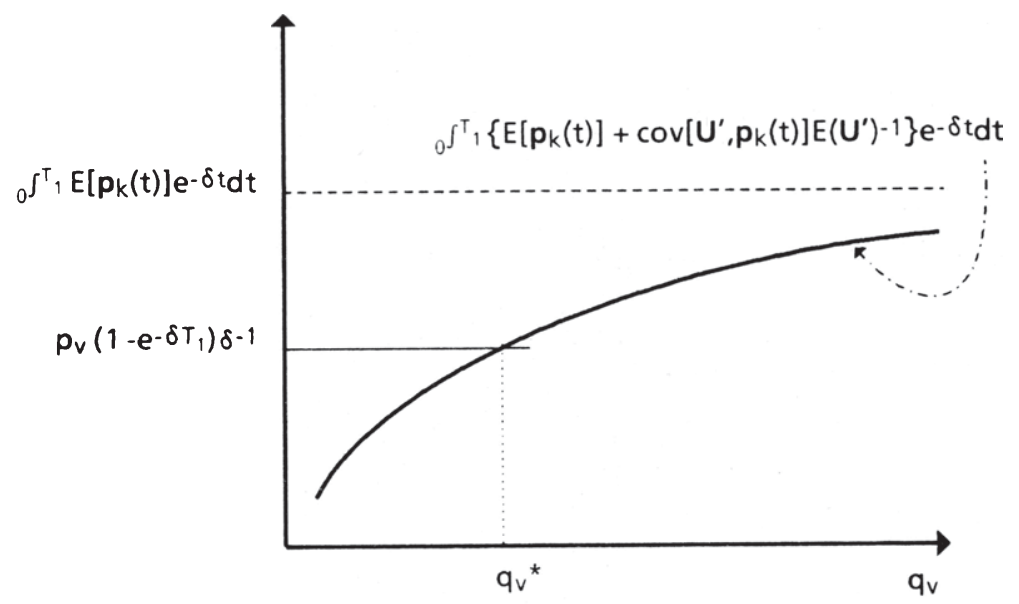

1) Vgl.zu einem àhnlichen Schaubild Roberts (1980), S.251. 
Je größer das Risiko auf dem Kassamarkt, desto größer die kontraktierte Menge.

Je höher die Erwartungen $\left\{E\left[p_{k}(t)\right]\right\}_{t}=0,1$. . T , desto kleıner die kontraktierte Menge.

Entsprechende Kombinationen von Erwartungshaltung und Risikoverhalten können dazu führen, daß für $\lambda=E\left(U^{\prime}\right)\left\{E\left[p_{k}(t)\right]+\operatorname{cov}\left[U^{\prime}, p_{k}(t)\right] E\left(U^{\prime}\right)-1\right.$ $\left.C^{\prime}(q(t))\right\} e^{-\delta t}$ gilt:

(6.26)(i) $E\left(U^{\prime}\right) T_{1}{ }^{-1} 0^{1} T^{T}\left[p_{v}-C^{\prime}(q(t))\right] e^{-\delta t} d t<\lambda$ und somit $q_{v}=0, q_{k}(t)=q(t)$ bzw.

(6.26)(ii) $E\left(U^{\prime}\right) T_{1}-1{ }_{0} \int^{T} 1\left[p_{v}-C^{\prime}(q(t))\right] e^{-\delta t} d t>\lambda$ und damit $q_{v}=q(t), q_{k}(t)=0$. Verträge werden für den Zeitraum $\left[0, T_{1}\right]$ nicht abgeschlossen, die gesamte Abbaumenge wird über die Kassamärkte abgesetzt, bzw. der Kassamarkt wird nicht beliefert, da die gesamte Ausbringung kontraktiert ist.

Die in 6.26 angesprochenen Abbauprofile als Randlösungen zeigt Schaubild 6.3.

Pfad A steht für das Abbauprofil ohne Vertragsabschlüsse. Die Abbautätigkeit wird in $T_{3}$ eingestellt. Pfad $B$ beschreibt das Abbauprofil mit Vertragsabschlüssen: Die gesamte Abbaumenge in $\left[0, T_{1}\right]$ ist kontraktiert. Nach Vertragsabschluß bietet das Abbauunternehmen wieder gemäß Pfad $A$ an. Hier stellt sich die Frage, ob der Abbauunternehmer in C oder in D auf Pfad A weiter abbaut. Baut er in $D$ weiter ab, dann ist sein Ressourcenbestand in $\mathrm{T}_{2}$ erschöpft und sein Angebot zu dem in $\mathrm{T}_{2}$ erwarteten Preis gleich null. Würde er in C den Abbaupfad A weiter verfolgen, so wäre bei einer noch als positiv geplanten Angebotsmenge in $T_{2}{ }^{\prime}$ sein Ressourcenlager bereits erschöpft.

Ein positiver Gegenwartswert des Schattenpreises läßt den Abbau solange andauern bis die Ressource erschöpft ist (vgl. 6.22 (iii)): 
Schaubild 6.3: Die intertemporale Ressourcenallokation mit und ohne Lieferverträge

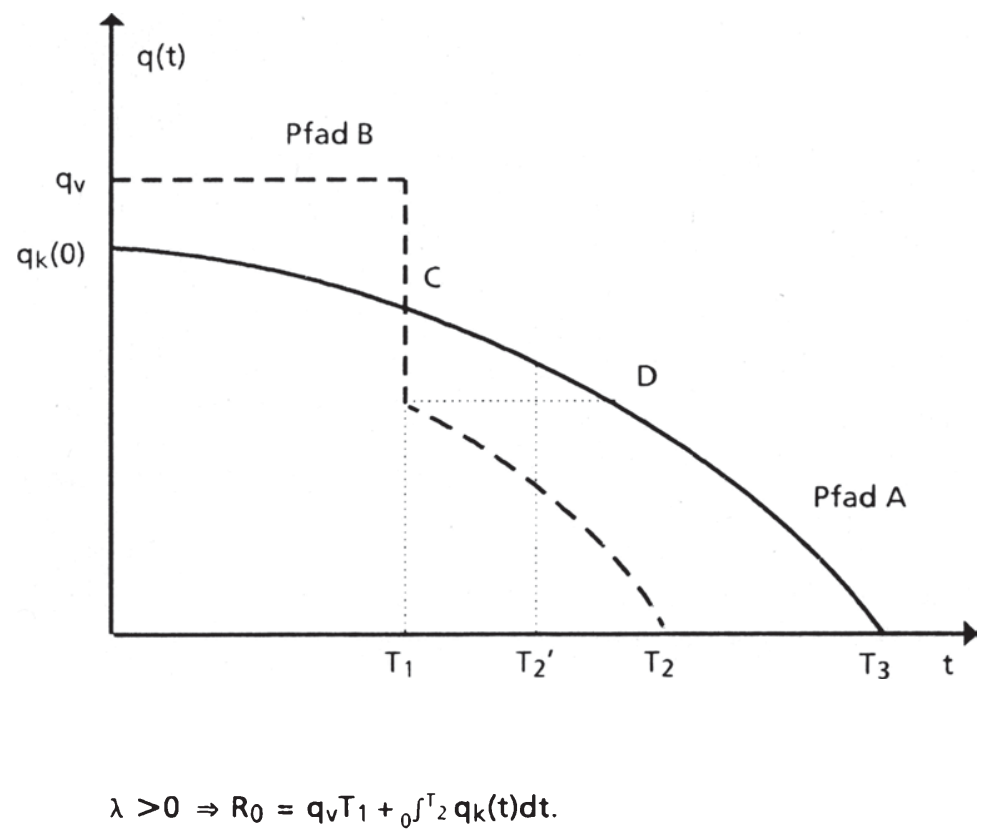

Es stellt sich weiter die Frage, ob das Ende der Vertragsdauer $T_{1}$ mit dem Erschöpfungszeitpunkt $T_{2}$ zusammenfallen kann.

Als Transversalitätsbedingung ergibt sich:

$$
\partial L / \partial T_{2}=E\left\{U^{\prime}\left[p_{k}\left(T_{2}\right) q_{k}\left(T_{2}\right)-C\left(q_{k}\left(T_{2}\right)\right)\right]\right\} e^{-\delta} T_{2}-\lambda q_{k}\left(T_{2}\right)=0
$$

Ersetzt man $\lambda$ durch 6.22 (ii), so folgt

$$
E\left\{U^{\prime}\left[p_{k}\left(T_{2}\right) q_{k}\left(T_{2}\right)-C\left(q_{k}\left(T_{2}\right)\right)\right]\right\}=E\left\{U^{\prime}\left[p_{k}\left(T_{2}\right)-C^{\prime}\left(q_{k}\left(T_{2}\right)\right)\right]\right\} q_{k}\left(T_{2}\right)
$$

und weiter

$$
C\left(q_{k}\left(T_{2}\right)\right)=C^{\prime}\left(q_{k}\left(T_{2}\right)\right) q_{k}\left(T_{2}\right)
$$


Der Ressourcenabbau wird eingestellt, wenn die Durchschnittskosten den Grenzkosten der letzten abgebauten Einheit entsprechen.

Sollte der Zeitpunkt $T_{1}$ Erschöpfungszeitpunkt sein, dann müßte dort obige Bedingung für die Abbaumengen erfüllt sein. D.h. es müßte gelten:

$$
E\left\{U^{\prime}\left[p_{v} q_{v}+p_{k}\left(T_{1}\right) q_{k}\left(T_{1}\right)-C\left(q_{k}\left(T_{1}\right)\right)\right]\right\} e^{-\delta T_{1}}=\lambda\left[q_{v}+q_{k}\left(T_{1}\right)\right] .
$$

Mit 6.22 (i) und (ii) für $\lambda$ wird dies zu

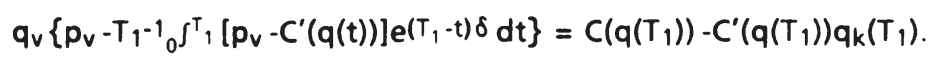

Erweitert man 6.30 mit - $C^{\prime}\left(q\left(T_{1}\right)\right) q_{v}$ und fordert die Gleichheit von Grenzund Durchschnittskosten, dann müßte erfüllt sein

$$
p_{v}-C^{\prime}\left(q\left(T_{1}\right)\right)=T_{1}-1{ }_{0} \int^{T_{1}}\left[p_{v}-C^{\prime}(q(t))\right] e^{\left(T_{1}-t\right) \delta d t,}
$$

was jedoch im Zeitpunkt $T_{1}$ nicht gilt, denn $p_{v}-C^{\prime}\left(q\left(T_{1}\right)\right)$ ist in laufenden Werten bei abnehmenden Abbaumengen (sinkenden Grenzkosten des Abbaus in der Zeit) der größte Grenzgewinn aus dem Vertragsverkauf der Ressource. Der Ressourcenabbau wird in $T_{1}$ nicht eingestellt.

Zur Herleitung der möglichen gleichgewichtigen Mengen- und Preispfade für den Kassamarkt und des Abbauprofils seien die folgenden Annahmen gemacht:

Die Angebotsseite setze sich aus einer Anzahl risikoaverser Ressourcenbesitzer zusammen, die eine bestimmte Zahl von Ressourcenlägern abbauen und rationale Erwartungen über die Nachfrage auf den zukünftigen Kassamärkten besitzen. Jeder Abbauunternehmer habe für den Zeitraum $\left[0, T_{1}\right]$ einen Teil seiner Abbaumengen kontraktiert. Weder auf der Angebotsnoch auf der Nachfrageseite werden Läger an abgebauten Ressourcen gehalten.

Das Nachfrageverhalten auf dem Kassamarkt wird sich im Zeitraum $\left[0, T_{1}\right]$ unterscheiden von dem im Zeitraum $\left(T_{1}, T_{2}\right]$ (Gleichung 6.18). Aufgrund von Vertragslieferungen wird die Nachfrage in $\left[0, T_{1}\right]$ geringer sein und einen elastischeren Verlauf aufweisen als in $\left(T_{1}, T_{2}\right]$. Je elastischer die Nachfrage 
wird, desto geringer werden die Preisveränderungen für eine bestimmte zeitliche Entwicklung des Kassaangebots sein.

Es gelte für die Nachfrage 1)

(6.32)(i) $\quad p_{k 1}(t)=N_{1}\left(q_{k}(t)\right) u_{1}(t)$ in $t \in\left[0, T_{1}\right]$ und

(6.32)(ii) $p_{k 2}(t)=N_{2}\left(q_{k}(t)\right) u_{2}(t)$ in $t \in\left(T_{1}, T_{2}\right]$,

mit $d N_{2} / d q_{k}=N_{2}{ }^{\prime}\left(q_{k}(t)\right)<d N_{1} / d q_{k}=N_{1}{ }^{\prime}\left(q_{k}(t)\right)<0, d^{2} N_{i} / d q_{k}=0$ füri $=1,2$ und $E\left[u_{1}(t)\right]=E\left[u_{2}(t)\right]=1$.

Über Gleichung 3.11 (\$.73) für die Abbaumengen und 3.12 für die Preise gelangt man zu den Schlußfolgerungen

$$
0>\mathrm{dq}_{2} / \mathrm{dt}>\mathrm{dq}_{1} / \mathrm{dt} \text { und } 0<\mathrm{dp}_{\mathrm{k}_{1} / \mathrm{dt}}<\mathrm{dp}_{\mathrm{k}_{2}} / \mathrm{dt} \text {. }
$$

$q_{1}(t)$ bezeichnet die Abbaumengen in $\left[0, T_{1}\right] ; q_{2}(t)$ bezeichnet die Abbaumengen in $\left(T_{1}, T_{2}\right]$.

Bieten die Abbauunternehmer im Zeitraum $\left[0, T_{2}\right]$ auf dem Kassamarkt an und beliefern sie ihre Vertragspartner im Zeitraum $\left[0, T_{1}\right]$, dann wird das folgende Abbauprofil erhalten.

Die Pfade A und B geben das Abbauprofil ohne und mit Lieferverträgen an.

1) Die Nachfragen werden hier als Inverse zu den auf S.174-176 abgeleiteten formuliert. Es gilt $N_{1}\left(q_{k}(t)\right)=g_{k}^{-1}\left\{E\left[p_{k}(t), p_{v}\right\}\right.$ für die Kassanachfrage während der Vertragsdauer und $N_{2}\left(q_{k}(t)\right)=g_{k}{ }^{-1}\left\{E\left[p_{k}(t)\right\}\right.$ nach Vertragsablauf. $u_{1}(t), u_{2}(t)$ sind exogene Störgrößen, welche den Prognosemodellen der Wirtschaftssubjekte zugrundeliegen. Für beide Nachfragen soll ein Preis existieren, beı dem die Nachfragemengen null werden. 
Schaubild 6.4: Die intertemporale Ressourcenallokation mit Lieferverträgen

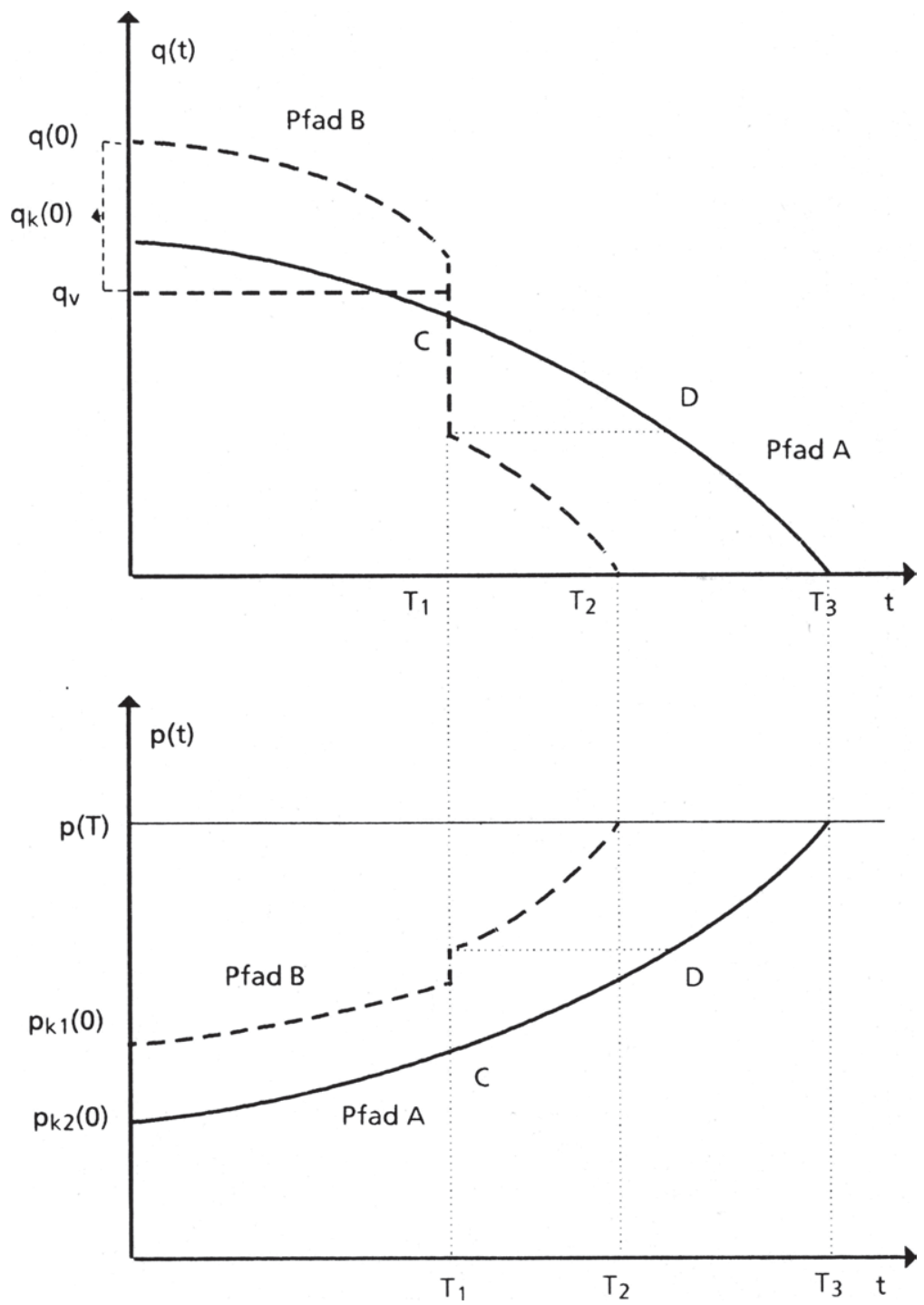


Da Vertragsabschlüsse das Risiko mindern 1), werden sich im Zeitraum [0, $\left.T_{1}\right]$ die Abbaumengen erhohen. Liefervertráge reduzıeren die Nachfrage und das Angebot für die Kassamärkte in diesem Zeitraum, so daß die Preisentwicklung auf einem höheren Niveau flacher verlàuft.2) Laufen die Verträge aus, dann sinkt die Abbaumenge auf das Niveau des Mengenpfades A in D. Der Preis auf dem Kassamarkt steigt auf das Niveau des Preispfades A in D. Von diesem Mengen- und Preisniveau aus läuft dann der Ressourcenabbau weiter, bis im Zeitpunkt $T_{2}$ die Abbautätigkeit eingestellt wird, weil die Ressource erschöpft ist und Nachfrage und Angebot beim Preis $P(T)$ null geworden sind.

Bei exogen vorgegebenem Zeitpunkt $T_{1}$ wird die Handelsmenge auf dem Kassamarkt in $T_{1}{ }^{3)}$ positiv sein, $d . h$. der Pfad B sinkt in $T_{1}$ nicht auf das Niveau $q_{v}$.

Betrachtet sei dazu Gleichung 6.30 in der äquivalenten Schreibweise 4)

$$
\begin{array}{r}
q_{v}\left\{p_{v}-C\left(q\left(T_{1}\right)\right) q\left(T_{1}\right)^{-1}-T_{1}-1{ }_{0} \int^{T},\left[p_{v}-C^{\prime}(q(t))\right] e^{\left.\left(T_{1}-t\right) \delta d t\right\}}=\right. \\
\left\{C\left(q\left(T_{1}\right)\right) q\left(T_{1}\right)^{-1}-C^{\prime}\left(q\left(T_{1}\right)\right)\right\} q_{k}\left(T_{1}\right) .
\end{array}
$$

Da in $T_{1}$ das Ressourcenlager noch nicht erschöpft ist, gilt $C\left(q\left(T_{1}\right)\right) q\left(T_{1}\right)^{-1}<$ $C^{\prime}\left(q\left(T_{1}\right)\right)$ und für $q_{k}\left(T_{1}\right), q_{v}>0$ weiter

$$
p_{v}-C\left(q\left(T_{1}\right)\right) q\left(T_{1}\right)^{-1}<T_{1}-1{ }_{0} \int^{T_{1}}\left[p_{v}-C^{\prime}(q(t))\right] e\left(T_{1}-t\right) \delta d t, d . h .
$$

der Durchschnittsgewinn aus dem Vertragsverkauf in $T_{1}$ ist kleiner als der auf $T_{1}$ kapitalisierte Grenzgewinn eıner Ressourceneinheit. Wäre $T_{1}$ nicht

1) Vgl. hierzu auch Abschnitt 5.1.

2) Der Preispfad B kann innerhalb $\left[0, T_{1}\right]$ den Preispfad A schneiden. Reagiert die Nachfrage auf die Eınführung von Lieferverträgen stärker (schwächer) als das Angebot, dann wird ein hoheres (niederes) Preis-

niveau als zuvor realisiert.

3) Linksseitige Annäherung an $T_{1}$.

4) Erhaltbar aus 6.30 mit der Zerlegung $C\left(q\left(T_{1}\right)\right)=C\left(q\left(T_{1}\right)\right)\left[q_{v}+q_{k}\left(T_{1}\right)\right] q\left(T_{1}\right)^{-1}$. 
exogen vorgegeben, so würde der Abbauunternehmer die Vertragsdauer erhohen wollen bis die Gleichheit gelten würde. Zu diesem Zeitpunkt würde dann für $q_{v}>0$ der Kassahandel $q_{k}\left(T_{1}\right)$ null werden. 1$)$

Gleichung 6.30' würde erhalten werden als Transversalitätsbedingung bei freier Vertragsdauer für $\mathrm{T}_{1}$.

In Schaubild 6.5 werden die Preis- und Mengenpfade für exogene und endogene Vertragszeiträume gegenúbergestellt.

Der Abschluß von Lieferverträgen führt also dazu, daß der in Abschnitt 3 für einen risikoaversen Abbauunternehmer festgestellte Allokationsbias zugunsten der Zukunft eine Korrektur zugunsten des Lieferzeitraumes, also der gegenwartsnäheren Perioden erfährt.

Was kann über die Auswirkung einer Änderung des Risikos oder der Risıkoaversion auf die Abbaumenge bzw. die Zusammensetzung des Angebots ausgesagt werden?

Eine komparativ dynamische Betrachtung2), ausgehend von den lotwendigen Bedingungen 6.22, führt zu folgendem Ergebnis:

Erhöht sich das Risiko oder die Risikoaversion, dann senkt der Ressourcenbesitzer seine Abbaumengen und führt gleichzeitig eine Umverteilung in der Zusammensetzung seines Angebots zugunsten der Vertragslieferungen durch. Die Vertragsmengen nehmen zu, das Kassaangebot nimmt ab.3) Mit geringeren Abbaumengen in jeder Periode erhöht sich der Abbauzeitraum. Der Schattenpreis (Gegenwartswert) der Ressource steigt mit dem Risiko.

1) Linksseitige Annäherung an $T_{1}$. Gleıchung 6.30' wäre für beide Seiten mit null erfüllt.

2) Vgl. Anhang V.

3) Ein weiteres Instrument zur Anpassung an ein erhöhtes Risiko, das dem Abbauunternehmer hier nıcht zur Verfügung steht, wäre die Länge des Vertragszeitraumes. 
Schaubıld 6.5: Die intertemporale Ressourcenallokation für exogene und endogene Vertragszeitraume

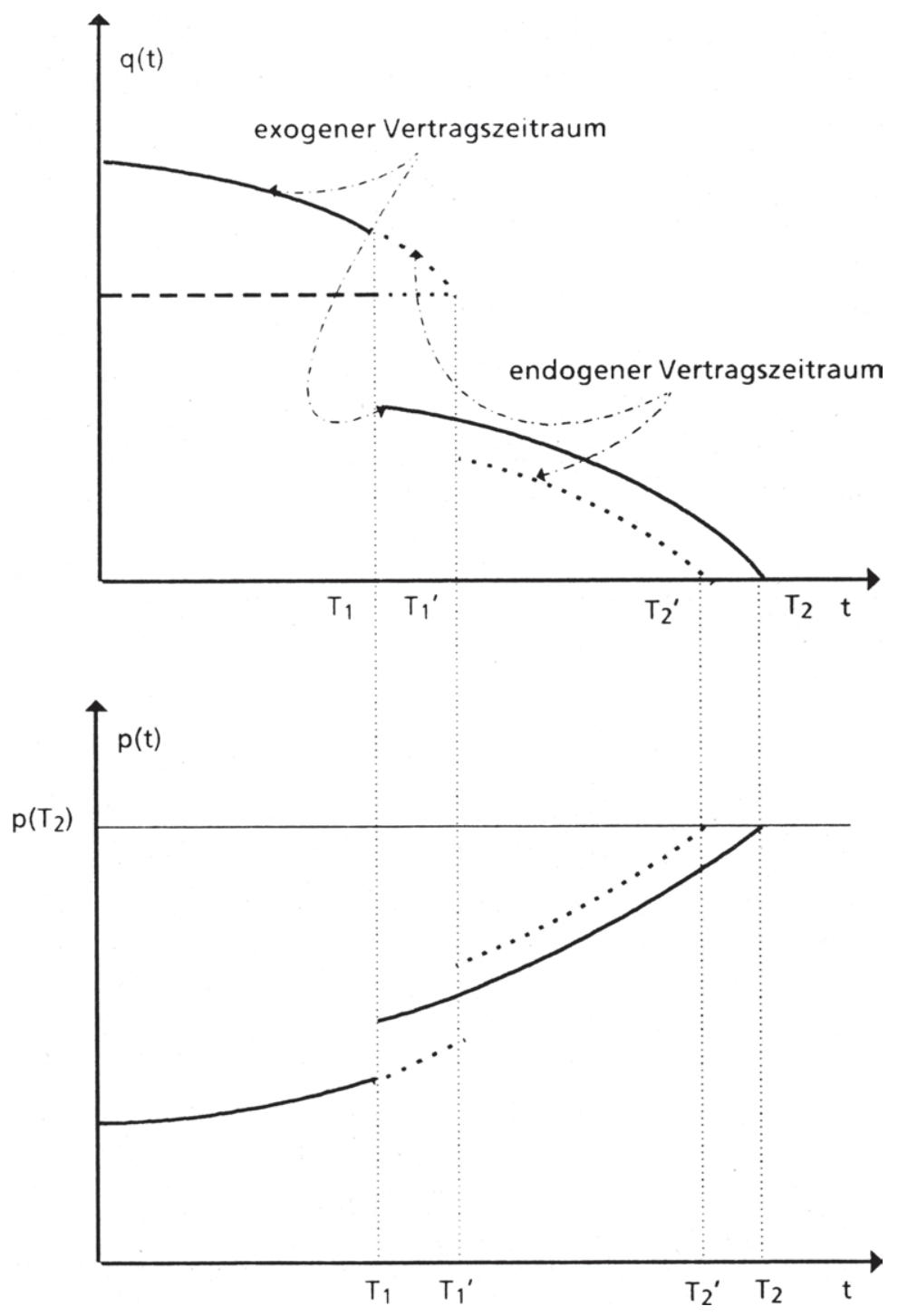




$$
\begin{aligned}
& d q_{v} / d \operatorname{cov}\left[U^{\prime}, p_{k}(t)\right]<0, d q_{k} / d \operatorname{cov}\left[U^{\prime}, p_{k}(t)\right]>0 \\
& d q / d \operatorname{cov}\left[U^{\prime}, p_{k}(t)\right]>0, d \lambda / d \operatorname{cov}\left[U^{\prime}, p_{k}(t)\right]<0 .
\end{aligned}
$$

Das Schaubild 6.6 stellt Preıs- und Mengenpfade mit verschiedenen Risıken gegenüber. Pfad A steht für ein höheres Rısiko als $P f a d B, d . h . \operatorname{cov}\left[U^{\prime}, p_{k}(t)\right]_{A}$ $<\operatorname{cov}\left[\mathrm{U}^{\prime}, \mathrm{p}_{\mathrm{k}}(\mathrm{t})\right]_{\mathrm{B}}<0$.

Für die Zeitpfade ermittelt man

(6.34) $\quad(d q / d t)_{B}<(d q / d t)_{A}<0$ und $(d q / d t)_{j}<(d q / d \tau)_{j}<0$ für $t \in\left[0, T_{1}\right], \tau \in\left(T_{1}, T_{2}\right], j=A, B$

(6.35) $\quad(d p / d t)_{B}>(d p / d t)_{A}>0$ und $0<(d p / d t)_{j}<(d p / d \tau)_{j}$ für $t \in\left[0, T_{1}\right], \tau \in\left(T_{1}, T_{2}\right], j=A, B$. 
Schaubild 6.6: Die intertemporale Ressourcenallokation mit Liefervertrágen bei unterschiedlichen Risiken

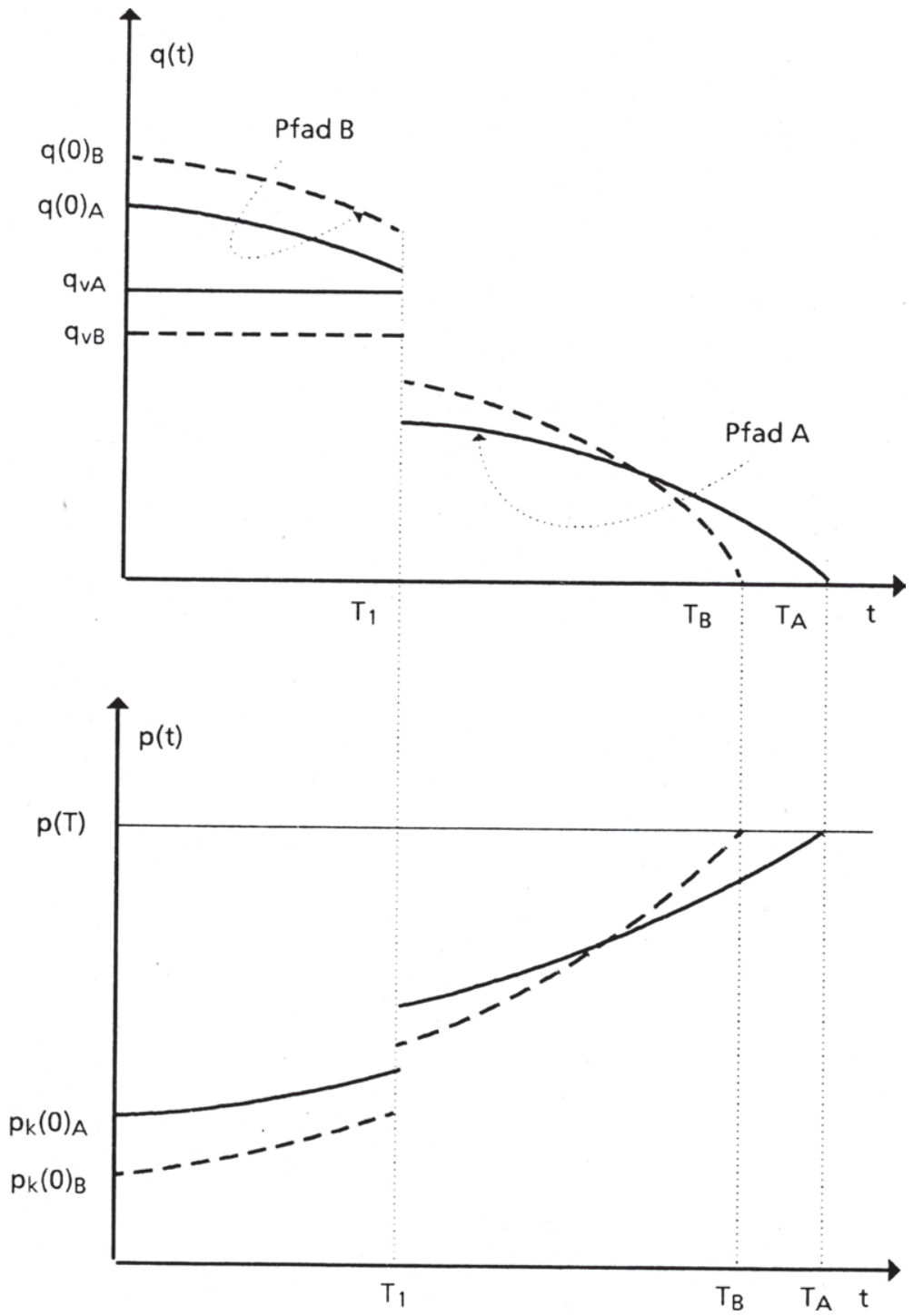




\subsubsection{Das Abbauprofil bei monopolisiertem Vertragshandel}

\subsubsection{Polypolistischer Kassamarkt}

Auf den Kassamärkten stehe der Abbauunternehmer weiter unter vollständiger Konkurrenz. Als einziger Anbieter von Lieferverträgen habe der betrachtete Abbauunternehmer die Möglichkeit, aus einer Menge von Lieferverträgen jene auszuwählen, die ihm nach Lieferpreis, Liefermenge und Vertragsdauer am geeignetesten erscheinen, seinen Erwartungsnutzen über dem Gesamtgewinn zu maximieren.

Für die Nachfrage nach Lieferverträgen soll gelten, 1)

$$
p_{v}=N_{v}\left(q_{v}, T_{1}\right) \text { mit } \partial N_{v} / \partial q_{v}=N_{q_{v}}{ }^{\prime}<0 \text { und } \partial N_{v} / \partial T_{1}=N_{T_{1}}{ }^{\prime}<0 \text {. }
$$

Je größer die Menge ist, die der Abbauunternehmer kontraktieren will, und je länger er vertraglich seinen Absatz sichern will, desto kleiner ist der Preis, den er vertraglich festschreiben kann.

Mit dieser Problemstellung wird der Gesamtgewinn des Abbauunternehmers zu

$$
\mathbf{G}={ }_{0} \int_{2}\left[N_{v}\left(q_{v_{v}} T_{1}\right) q_{v}+p_{k}(t) q_{k}(t)-C(q(t))\right] e^{-\delta t d t} .
$$

Das Optimierungsproblem lautet nun:

1) Vgl.zu dieser Annahme Roberts (1980), S.249. 
(6.36)

$$
\begin{aligned}
& \max E[U(G)] \\
& \left(q_{v}, q_{k}(t), T_{1}, T_{2}\right) \\
& \text { u.d.B. } \quad R_{0} \geq q_{v} T_{1}+{ }_{0} \int^{T}{ }_{2} q_{k}(t) d t, R_{0} \text { gegeben, } \\
& \qquad \begin{aligned}
q_{k}(t) & \geq 0 \text { für } t \in\left[0, T_{2}\right] \\
q_{v} & \geq 0 \text { für } t \in\left[0, T_{1}\right] \\
q_{v} & =0 \text { für } t \in\left(T T_{1}, T_{2}\right] .
\end{aligned}
\end{aligned}
$$

Es ergeben sich die notwendigen Bedingungen

(6.37)(i) $E\left(U^{\prime}\right)\left\{_{0} J^{T_{1}}\left[p_{v}+q_{v} N_{q_{v}}{ }^{\prime}-C^{\prime}(q(t))\right] e^{-\delta t} d t\right\}-\lambda T_{1}\left\{\begin{array}{lll}=0 & & q_{v}>0 \\ & \text { für } & \\ \leq 0 & & q_{v}=0\end{array}\right.$

(6.37)(ii) $E\left\{U^{\prime}\left[p_{k}(t)-C^{\prime}(q(t))\right]\right\} e^{-\delta t}-\lambda$

$$
\begin{aligned}
& \begin{cases}=0 & \\
\text { für } & q_{k}(t)>0 \\
\leq 0 & q_{k}(t)=0\end{cases} \\
& \begin{cases}=0 & \lambda>0 \\
\text { für } & \\
\geq 0 & \lambda=0\end{cases}
\end{aligned}
$$

(6.37)(iv) $E\left\{U^{\prime}\left\{q_{v} N_{T_{1}}{ }^{\prime} \int^{T_{1}} e^{-\delta t} d t+\left\{p_{v} q_{v}+p_{k}\left(T_{1}\right) q_{k}\left(T_{1}\right)-C\left(q\left(T_{1}\right)\right)\right] e^{-\delta T_{1}}\right\}\right\}-$ $\lambda q\left(T_{1}\right)=0$

$(6.37)(v) \quad E\left\{U^{\prime}\left[p_{k}\left(T_{2}\right) q_{k}\left(T_{2}\right)-C\left(q_{k}\left(T_{2}\right)\right)\right]\right\} e^{-\delta} T_{2}-\lambda q_{k}\left(T_{2}\right)=0$. 
Im Vergleich mit dem Optimierungsproblem 6.21 andert sich nun die notwendige Bedingung (i) und es kommt die Bedingung (iv) für die Instrumentvariable $T_{1}$ hinzu. 6.37 (i) kann umgeschrieben werden zu 1)

$$
{ }_{0} \int^{T}{ }_{1}\left[p_{v}\left(1+\eta_{v}-1\right)\right] e^{-\delta t} d t=\lambda T_{1} E\left(U^{\prime}\right)-1+{ }_{n} \int^{T}: C^{\prime}(q(t)) e^{-\delta t} d t .
$$

Mit dieser Umformung und dem Einsetzen von

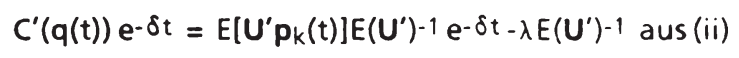

ergibt sich

$$
\begin{aligned}
& { }_{0}^{\int^{T} 1}\left[p_{v}\left(1+n_{v}{ }^{-1}\right)\right] e^{-\delta t} d t= \\
& \lambda T_{1} E\left(U^{\prime}\right)^{-1}+{ }_{0} \int^{T}{ }_{1}\left\{E\left[U^{\prime} p_{k}(t)\right] E\left(U^{\prime}\right)^{-1} e^{-\delta t}-\lambda E\left(U^{\prime}\right)^{-1}\right\} d t,
\end{aligned}
$$

und weiter

$$
{ }_{0}^{S^{\top} 1}\left[p_{v}\left(1+n_{v}-1\right)\right] e^{-\delta t} d t={ }_{0} S^{\top} 1 E\left[U^{\prime} p_{k}(t)\right] E\left(U^{\prime}\right)-1 e^{-\delta t} d t .
$$

Beachtet man, daß $p_{v}\left(1+n_{v}{ }^{-1}\right)$ in $\left[0, T_{1}\right]$ konstant ist und benutzt man die Definition für $\operatorname{cov}\left[\mathbf{U}^{\prime}, \mathrm{p}_{k}(\mathrm{t})\right]$, dann gilt

$$
\begin{aligned}
& {\left[p_{v}\left(1+n_{v}-1\right)\right]\left(1-e^{-\delta T_{1}}\right) \delta-1=} \\
& { }_{0} \int^{T}\left\{E\left[p_{k}(t)\right]+\operatorname{cov}\left[U^{\prime}, p_{k}(t)\right] E\left(U^{\prime}\right)-1\right\} e^{-\delta t d t .}
\end{aligned}
$$

Der Gegenwartswert der Grenzeinnahmen über dem Vertragszeitraum muß gleich sein dem Gegenwartswert der um einen Risikoabschlag verminderten Erwartungswerte für die Preise auf den Kassamärkten dieses Zeitraumes.2)

Schaubild 6.7 zeigt die Lösung von 6.40 für ein gegebenes Abbauprofil.

1) Für $q_{v}>0, q_{k}(t)>0$. Es gilt $\eta_{v}=\left(d q_{v} / d p_{v}\right) p_{v} q_{v}{ }^{-1}$.

2) Dieses Ergebnis ist auch bei Roberts (1980), S.250 zu finden. Vgl. hierzu auch sein Schaubild S.251, das die Lösung nicht adäquat wiedergibt. 
Schaubıld 6.7: Die optımale vertragliche Liefermenge bei wahlbarer Vertragsdauer

Erlös in Gegenwartswerten

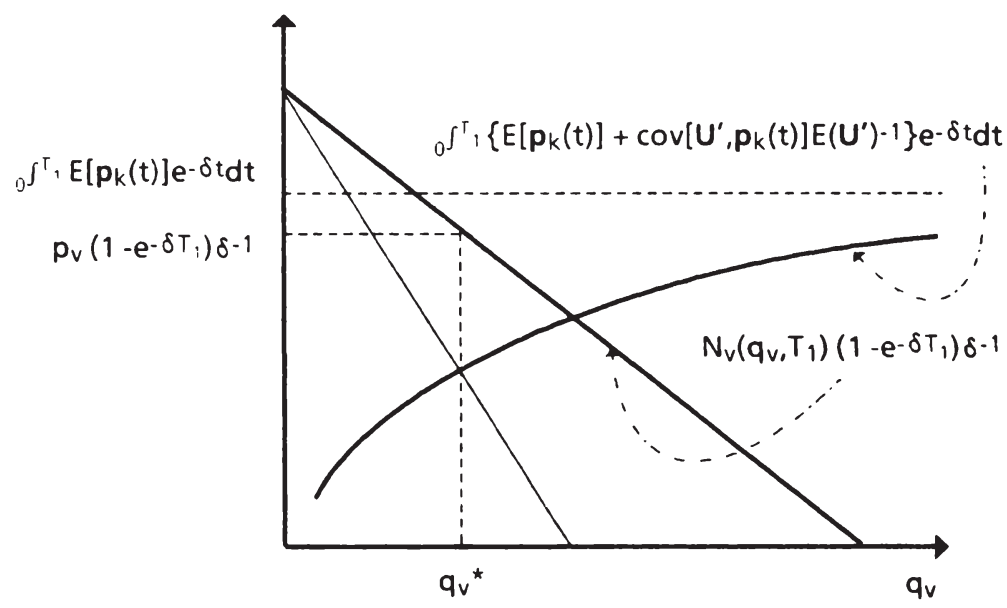

Eine Erhohung des Risikos auf dem Kassamarkt führt bei gegebener Vertragsdauer zu einem geringeren Lieferpreıs. Denn eine Rısikoerhöhung läßt den Abbauunternehmer eine Umverteilung seines Ressourcenangebotes zugunsten der Vertragslieferungen vornehmen. Höhere Vertragsmengen konnen aber nur zu niedrigeren Lieferpreisen abgesetzt werden.

In einer komparativ dynamischen Analyse wäre zu zeigen, wie sich eine Variation des Risikos auswirkt auf die Kontraktmenge, das Kassaangebot, die Abbaumenge, den Schattenpreis der Ressource, die Vertragsdauer und den Zeitpunkt der Erschöpfung des Ressourcenlagers. Jedoch liefert eine solche Analyse keine eindeutigen Aussagen (Anhang VI).

Die notwendige Bedingung 6.37 (iv) macht eine Aussage über die optimale Länge des Vertragszeitraumes. 
Es folgt zunàchst:

$$
\begin{aligned}
& q_{v} N_{T_{1}}{ }_{1}{ }^{T}{ }^{T} e^{-\delta t} d t+ \\
& \left.\left\{p_{v} q_{v}+E\left[U^{\prime} p_{k}\left(T_{1}\right)\right] E\left(U^{\prime}\right)-1 q_{k}\left(T_{1}\right)-C\left(q\left(T_{1}\right)\right)\right]\right\} e^{-\delta T_{1}=\lambda q\left(T_{1}\right) E\left(U^{\prime}\right)-1 .}
\end{aligned}
$$

Setzt man $q\left(T_{1}\right)=q_{v}+q_{k}\left(T_{1}\right)$ und ersetzt dann $\lambda$ einmal nach 6.37 (i) und einmal nach (ii), so ergibt sich

$$
\begin{aligned}
& q_{v} N_{T_{1}}{ }_{1} \int^{T_{1}} e^{-\delta t} d t+
\end{aligned}
$$

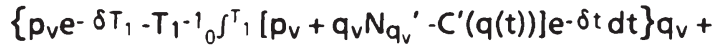

$$
\begin{aligned}
& C^{\prime}\left(q\left(T_{1}\right)\right) q_{k}\left(T_{1}\right) e^{-\delta T_{1}-C\left(q\left(T_{1}\right) e^{-} \delta T_{1}=0 .\right.} \text {. }
\end{aligned}
$$

Für $C\left(q\left(T_{1}\right)\right)$ kann geschrieben werden

$$
C\left(q\left(T_{1}\right)\right)=q_{v} q\left(T_{1}\right)^{-1} C\left(q\left(T_{1}\right)\right)+q_{k}\left(T_{1}\right) q\left(T_{1}\right)^{-1} C\left(q\left(T_{1}\right)\right) .
$$

Damit wird $6.42 \mathrm{zu}$

$$
\begin{aligned}
& \left\{\left[p_{v}-C\left(q\left(T_{1}\right)\right) q\left(T_{1}\right)^{-1}\right] e^{-\delta} T_{1}-\right. \\
& \left.T_{1}{ }^{-1} 0^{T}{ }_{1}\left[p_{v}\left(1+\eta_{v}-1\right)-C^{\prime}(q(T))\right] e^{-\delta t} d t\right\} q_{v}+ \\
& {\left[C^{\prime}\left(q\left(T_{1}\right)\right)-C\left(q\left(T_{1}\right)\right) q\left(T_{1}\right)^{-1}\right] e^{-\delta T_{1}} q_{k}\left(T_{1}\right)=-q_{v} N_{T_{1}}{ }_{0}{ }^{\prime} T^{T} e^{-\delta t} d t .}
\end{aligned}
$$

Auf der linken Seite von Gleichung 6.43 kommt zu stehen in Gegenwartswerten:

Durchschnittsgewinn aus dem Vertragsverkauf in $T_{1}$ minus Grenzgewinn aus dem Vertragsverkauf plus Durchschnittsgewinn aus dem Kassaverkauf minus Grenzgewinn aus dem Kassaverkauf in $T_{1}$.

Die optimale Vertragsdauer ist erreicht, wenn eine Minderung der Grenzeinnahme aus dem Vertragsverkauf aufgrund einer Vertragsverlängerung der Summe der oben genannten Differenzen der Durchschnittsgewinne aus den beiden Verkaufsarten gleich ist.

Betrachtet man diese Bedingung unter der Annahme $N_{T_{1}}{ }^{\prime}=0$, dann wird gefordert, daß die (negative) Differenz aus Durchschnitts- und Grenzgewinn des Vertragsverkaufs gleich der Differenz aus Durchschnitts- und Grenzgewinn aus dem Kassaverkauf in $T_{1}$ sein soll. Der Vertragszeitraum vergrößert sich. Solange die Differenz aus Durchschnitts- und Grenzgewinn 
des Vertragsverkaufs absolut großer als die Differenz aus Durchschnittsund Grenzgewinn des Kassaverkaufs ist, wird der Abbauunternehmer den Vertragszeitraum ausdehnen. In $T_{1}$ ist das Kassaangebot (beı linksseıtiger Annäherung) auf null gesunken. Der Durchschnıtts- und der Grenzgewinn aus dem Vertragsverkauf sınd dann gleich. Denn aus 6.43 erhält man unter

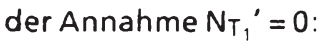

$$
\left[p_{v}-C\left(q\left(T_{1}\right)\right) q\left(T_{1}\right)^{-1}\right] e^{-\delta T_{1}}=T_{1}{ }^{-1}{ }_{0} \int^{T_{1}}\left[p_{v}\left(1+\eta_{v}-1\right)-C^{\prime}(q(T))\right] e^{-\delta t} d t .
$$

In Schaubild 6.8 kennzeichnet Pfad A das geplante Abbauverhalten ohne Vertragsabschlüsse. Pfad B (C) gibt das Abbauverhalten unter der Annahme $\mathrm{N}_{\mathrm{T}_{1}}{ }^{\prime}<0(=0)$ an.

Schaubild 6.8: Die intertemporale Ressourcenallokation in Abhängigkeit von dem Einfluß der Vertragsdauer auf die Nachfrage

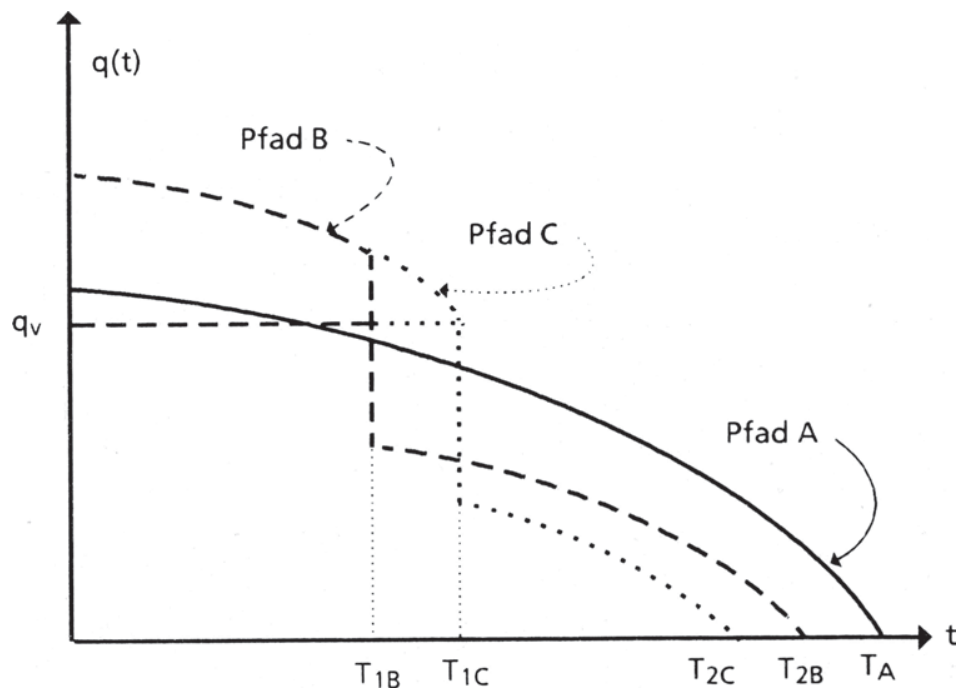


Eın Vergleich der Ergebnısse zwischen polypolistıschem Vertragshandel (gekennzeichnet durch den Index $p$ ) und monopolistischem Vertragshandel (Index $m$ ) führt zu den folgenden Aussagen: 1)

(1) Wählt der Monopolist einen zumındest gleıch langen Vertragszeitraum wie der Polypolist $\left(T_{1 m} \geq T_{1 p}\right)$, dann fordert der Monopolist bei geringeren Periodenliefermengen einen höheren Vertragspreis $\left(p_{v m}>p_{v p}, q_{v m}<q_{v p}\right.$ ) (Gleichungen 6.25 und 6.40).

(2) Wählt der Monopolist einen kürzeren Vertragszeitraum als der Polypolist $\left(T_{1 m}<T_{1 p}\right)$, dann fordert er je nach Preiselastizität der Nachfrage nach Liefermengen $n_{v}$,

(a) für $\eta_{v} \rightarrow-1$, bei geringeren Periodenliefermengen einen höheren Vertragspreis $\left(p_{v m}>p_{v p}, q_{v m}<q_{v p}\right.$ ).

(b) für $\eta_{v} \rightarrow-\infty$, bei größeren Periodenliefermengen einen geringeren Vertragspreis $\left(p_{v m}<p_{v p}, q_{v m}>q_{v p}\right)$.

(3) Da sich der Abbauunternehmer auf den Kassamärkten unter vollständiger Konkurrenz befindet, gelten für die zeitliche Veränderung der Abbaumengen und der Kassapreise in den beiden Zeiträumen $\left[0, T_{1}\right]$ und $\left(T_{1}, T_{2}\right]$ dieselben Ungleichungen wie im Abschnitt zuvor ( $v g l$.

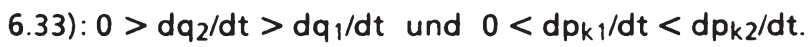

Die Pfade für die Mengen und Preise unterscheiden sich von denen im Abschnitt zuvor jedoch im Niveau.

Wählt der monopolistische Abbauunternehmer einen Vertragszeitraum $T_{1 m} \leq T_{1 p}$ mit $q_{v m}<q_{v p}$, dann verbleibt für den Abbauzeitraum $\left(T_{1 m}, T_{2 m}\right.$ ] eine größere Handelsmenge für die Kassamärkte, wenn nicht der Rückgang an Vertragsmengen kompensiert wird durch die Zunahme der Handelsmengen auf den Kassamärkten in [0, $T_{1 \mathrm{~m}}$ ]. Der Abbaupfad für den Zeitraum $\left(T_{1 m}, T_{2 m}\right)$ verläuft auf einem höheren, der Preispfad auf einem niedrigeren

1) In beiden Fällen werden für die Abbauunternehmer die gleiche Erwartungshaltung und das gleiche Risikoverhalten unterstellt. 
Niveau als bei vollständiger Konkurrenz. Je nach Reaktion der Nachfrage beim Übergang zu einem monopolisierten Vertragsmarkt, kann das Niveau des Preispfades in $\left[0, T_{1 m}\right]$ über oder unter dem des Pfades bei vollständiger Konkurrenzliegen.

Schaubild 6.9 gibt die Allokationsergebnisse bei angebotsmonopolistischem und -polypolistischem Vertragshandel an.

Wählt der monopolistische Abbauunternehmer seinen Vertragszeitraum so, daß $T_{1 m}<T_{1 p}$ und $q_{v m}>q_{v p}$ oder $T_{1 m}>T_{1 p}$ und $q_{v m}<q_{v p}$ ist, dann sind vergleichende Aussagen nur durch zusätzliche Annahmen möglich.

\subsubsection{Monopolistischer Kassamarkt}

Der Abbauunternehmer werde nun als alleiniger Anbieter der Rohstoffe sowohl auf den Kassamärkten als auch auf dem Vertragsmarkt betrachtet. In die Planung seines Abbauprogrammes werden nun explizit die erwarteten Nachfragen der Kassamärkte eingehen. Unterstellt seien die Nachfragefunktionen 6.32 (i) und (ii). Als Gegenwartswert seiner Gewinne ermittelt der Monopolist

$$
G={ }_{0} \int_{2}\left[N_{v}\left(q_{v}, T_{1}\right) q_{v}+p_{k}(t) q_{k}(t)-C(q(t))\right] e^{-\delta t d t}
$$

und maximiert darüber den Erwartungsnutzen.

$$
\begin{aligned}
& \max \quad E[U(G)] \\
& \left(q_{v}, q_{k}(t), T_{1}, T_{2}\right) \\
& \text { u.d.B. } \quad R_{0} \geq q_{v} T_{1}+{ }_{0} \int^{T} q_{k}(t) d t, R_{0} \text { gegeben, } \\
& q_{k}(t) \geq 0 \text { für } t \in\left[0, T_{2}\right] \text {, } \\
& q_{v} \geq 0 \text { für } t \in\left[0, T_{1}\right], q_{v}=0 \text { für } t \in\left(T_{1}, T_{2}\right] \text {, } \\
& p_{k 1}(t)=N_{1}\left(q_{k}(t)\right) u_{i}(t), ı=1,2
\end{aligned}
$$


Schaubild 6.9: Die intertemporale Ressourcenallokatıon bei verschiedenen Marktformen des Vertragshandels

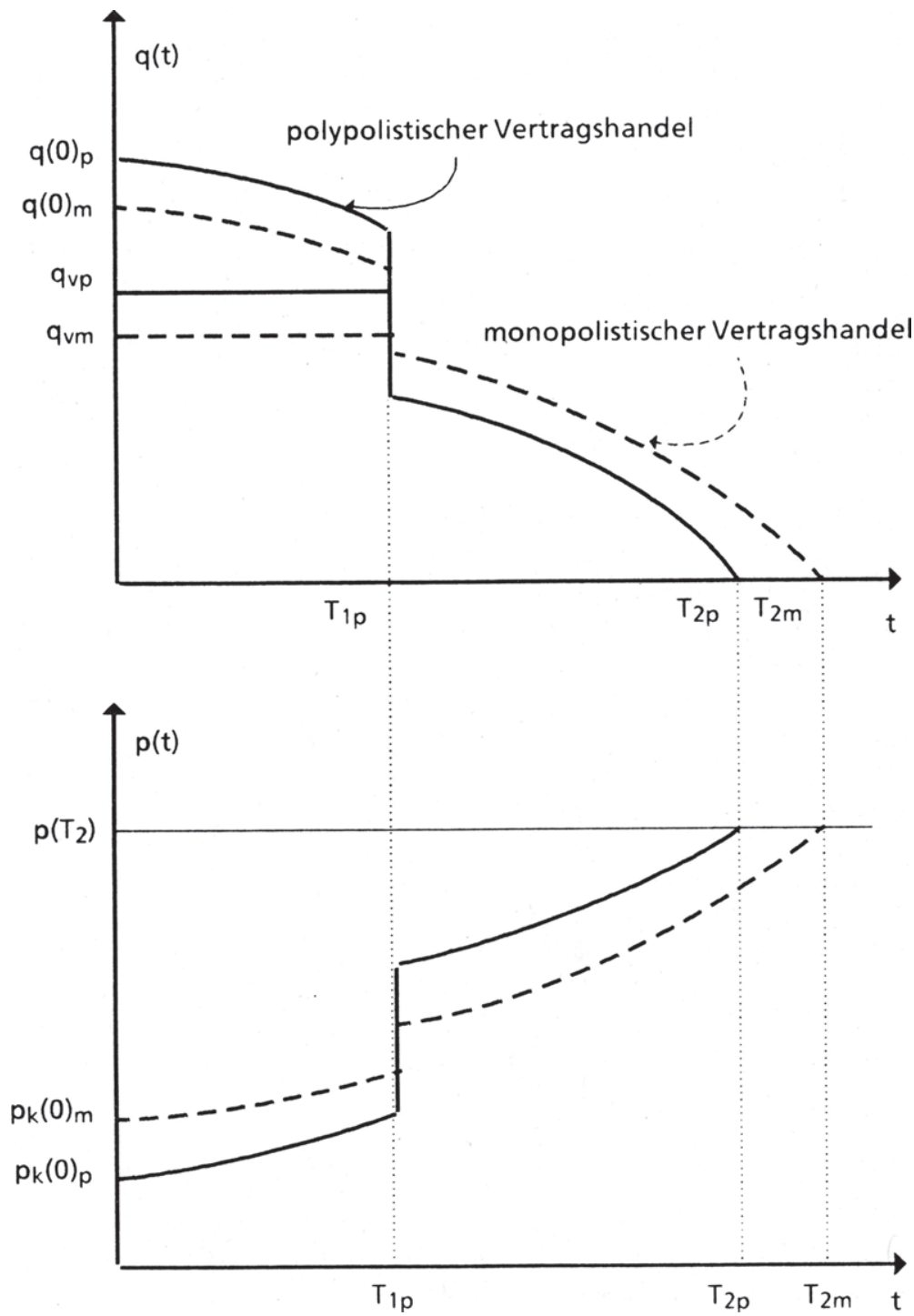


Mittels der Lagrangefunktion

$$
L=E[U(G)]+\lambda\left[R_{0}-q_{v} T_{1}-{ }_{0} \int_{2} q_{k}(t) d t\right]
$$

wird an notwendigen Bedingungen erhalten

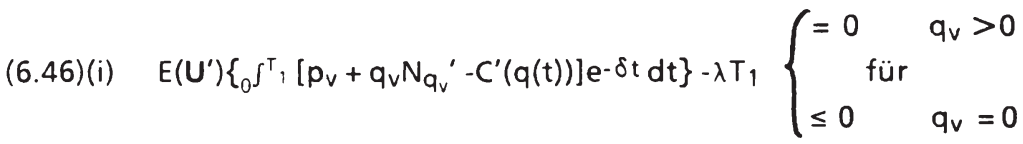

(6.46)(ii) $E\left\{U^{\prime}\left[p_{k i}(t)+q_{k}(t) N_{i}{ }^{\prime}\left(q_{k}(t)\right) u_{i}-C^{\prime}(q(t))\right]\right\} e^{-\delta t-\lambda} \begin{cases}=0 & q_{k}(t)>0 \\ \text { für } & \\ \leq 0 & q_{k}(t)=0\end{cases}$

(6.46)(iii) $R_{0}-q_{v} T_{1}-{ }_{0} T_{2} q_{k}(t) d t$

$$
\left\{\begin{array}{lll}
=0 & \lambda>0 \\
\text { für } & \\
\geq 0 & \lambda=0
\end{array}\right.
$$

(6.46)(iv) $E\left\{U^{\prime}\left\{q_{v} N_{T_{1}}{ }_{0} \int^{T_{1}} e^{-\delta t} d t+\left[p_{v} q_{v}+p_{k_{1}}\left(T_{1}\right) q_{k}\left(T_{1}\right)-C\left(q\left(T_{1}\right)\right)\right] e^{-\delta T_{1}}\right\}\right\}-$

$$
\lambda q\left(T_{1}\right)=0
$$

$(6.46)(v) \quad E\left\{U^{\prime}\left[p_{k 2}\left(T_{2}\right) q_{k}\left(T_{2}\right)-C\left(q_{k}\left(T_{2}\right)\right)\right]\right\} e^{-\delta} T_{2}-\lambda q_{k}\left(T_{2}\right)=0$

Hierin ist für die Nachfrage auf den Kassamärkten zu beachten,

$$
p_{k_{1}}(t)=\left\{\begin{array}{lll}
N_{1}\left(q_{k}(t)\right) u_{1}(t) & t \in\left[0, T_{1}\right] \\
N_{2}\left(q_{k}(t)\right) u_{2}(t) & \text { für } & t \in\left(T_{1}, T_{2}\right]
\end{array}\right.
$$

Es sei $n_{k}{ } e(t)-1=N_{i}{ }^{\prime}\left(q_{k}(t)\right) q_{k}(t) N_{1}\left(q_{k}(t)\right)-1$ die erwartete Preiselastitzität der Nachfrage auf dem Kassamarkt imZeitpunkt $t$. 
Aus (i) und (ii) werden im Zeitraum $\left[0, T_{1}\right]$ bei positiven Handelsmengen $q_{v}$, $q_{k}(t)$ für das Optimum erhalten:

$$
E\left(\mathbf{U}^{\prime}\right) T_{1}{ }^{-1}{ }_{0} \int^{T_{1}}\left[p_{v}\left(1+n_{v^{-1}}\right)-C^{\prime}(q(t))\right] e^{-\delta t} d t=\lambda \text { und }
$$

(6.47)(ii) $E\left(\mathbf{U}^{\prime}\right)\left\{\left\{E\left[p_{k i}(t)\right]+\operatorname{cov}\left[\mathbf{U}^{\prime}, p_{k i}(t)\right] E\left(U^{\prime}\right)-1\right\}\left(1+n_{k i} e^{-1}\right)-C^{\prime}(q(t))\right\}=\lambda e^{\delta t}$,

$$
\text { mit } i=1 \text { für } t \in\left[0, T_{1}\right] \text { und } i=2 \text { für } t \in\left(T_{1}, T_{2}\right] \text {. }
$$

Beide Gleichungen liefern für das optimale Angebotsverhalten im Vertragsund Kassahandel die Aussage, daß im Erwartungsnutzenmaximum die Gleichheit der Barwerte der Grenzerlöse aus dem Vertrags- und dem Kassaverkauf der Ressource über den Vertragszeitraum gilt:

$$
\begin{aligned}
& p_{v}\left(1+n_{v}-1\right)\left(1-e^{-\delta} T_{1}\right) \delta-1= \\
& { }_{0}^{S^{T} 1}\left\{E\left[p_{k 1}(t)\right]+\operatorname{cov}\left[U^{\prime}, p_{k 1}(t)\right] E\left(U^{\prime}\right)^{-1}\right\}\left(1+n_{k 1} e^{-1}\right) e^{-\delta t} d t
\end{aligned}
$$

Das monopolistische Angebotsverhalten auf den Kassamärkten führt im Vergleich zum polypolistischen Angebotşverhalten bei der gleichen Erwartungshaltung und dem gleichen Risikoverhalten zu geringeren Handelsmengen, 1) d.h. der Monopolist plant für die zeitliche Entwicklung seiner Handelsmengen auf den Kassamärkten in den Zeiträumen $\left[0, T_{1}\right]$ und $\left(T_{1}, T_{2}\right]$ ein niedrigeres Niveau mit flacherem Verlauf.2) Bei unveränderten Vertragshandelsmengen (des vorigen Abschnitts) würde der Ressourcenbestand später erschöpft werden.

Für die Transversalitätsbedingung 6.46 (iv) ergibt sich mit 6.46 (i) und (ii) für den Zeitpunkt $T_{1}$ :

$$
\begin{aligned}
& \left\{\left[p_{v}-C\left(q\left(T_{1}\right)\right) q\left(T_{1}\right)^{-1}\right] e^{-\delta} T_{1}-\right. \\
& \left.T_{1}-1{ }_{0} \delta^{T_{1}}\left[p_{v}\left(1+\eta_{v}-1\right)-C^{\prime}(q(T))\right] e^{-\delta t} d t\right\} q_{v}+ \\
& \left\{C^{\prime}\left(q\left(T_{1}\right)\right)-\left\{E\left[p_{k 1}\left(T_{1}\right)\right]+\operatorname{cov}\left[U^{\prime}{ }_{1} p_{k 1}\left(T_{1}\right)\right] E\left(U^{\prime}\right)^{-1}\right\} \eta_{k 1} e^{-1}-\right. \\
& \left.C\left(q\left(T_{1}\right)\right) q\left(T_{1}\right)^{-1}\right\} e^{-} \delta T_{1} q_{k}\left(T_{1}\right)=-q_{v} N_{T_{1}}{ }_{0}{ }^{T_{1}} e^{-\delta t} d t .
\end{aligned}
$$

1) Vgl. Abschnitt 3.2.1.

2) Die Handelsprofile auf den Kassamärkten sind formal äquivalent dem Ergebnis in Gleichung 3.21 für nun unterschiedliche Nachfrageverläufe auf den Kassamärkten in $\left[0, T_{1}\right]$ bzw. $\left(T_{1}, T_{2}\right]$. 
Auch hier gilt die zuvor gemachte Aussage, daß die optimale Vertragsdauer dann erreicht ist, wenn die Summe der Durchschnittsgewinne aus dem Vertrags- und Kassaverkauf der Rohstoffe gleich ist der Summe der Grenzgewinne aus den beiden Handelsarten, abzüglich der Minderung der Grenzeinnahmen aus dem Vertragsverkauf aufgrund einer Vertragsverlängerung zu diesem Zeitpunkt um eine Periode.

6.49 unterscheidet sich von 6.43 durch den anders lautenden Grenzgewinn des Monopolisten. Wählt man für die linke Seite der Gleichung 6.49 dieselbe Schreibart wie in 6.43 , so wird für die rechte Seite von 6.49 erhalten

$$
\begin{aligned}
\ldots= & -q_{v} N_{T_{1}}{ }_{1}{ }^{T}{ }_{1} e^{-\delta t} d t+ \\
& \left\{E\left[p_{k 1}\left(T_{1}\right)\right]+\operatorname{cov}\left[U^{\prime}, p_{k 1}\left(T_{1}\right)\right] E\left(U^{\prime}\right)^{-1}\right\} \eta_{n_{1} 1} e^{-1} e^{-\delta T}, .
\end{aligned}
$$

Aufgrund seiner monopolistischen Stellung am Kassamarkt1) wählt der Abbauunternehmer nun einen größeren Vertragszeitraum. Der größere Vertragszeitraum kann einhergehen mit einer größeren Periodenliefermenge bei geringeren Lieferpreisen oder, was eher der Fall sein dürfte, mit geringeren Periodenliefermengen bei höheren Lieferpreisen. Eine genaue Aussage über den Verglech mit dem vorigen Abschnitt läßt sich für die Vertragsmengen und -preise nicht machen.

Die Transversalitätsbedingung 6.46 (v) liefert wie 3.23 die Aussage, daß der Ressourcenbestand über einen größeren Zeitraum abgebaut wird.

\subsubsection{Verträge mit unterschiedlicher Laufzeit}

Betrachtet sei der Abbauunternehmer des Abschnitts 6.1.3.1., der auf dem Kassamarkt unter vollständiger Konkurrenz steht, für den Vertragshandel

1) Vgl. hierzu auch 3.23 
jedoch der einzige Anbieter ist.1) Im Vertragshandel sel er zusätzlich in der Lage, zwischen den einzelnen Abnehmern preislich zu differenzieren.

Für die N rohstoffverarbeitenden Unternehmen gelten die Nachfragefunktionen

$$
\begin{aligned}
p_{v 1}=N_{v 1}\left(q_{v 1}, T_{1}\right) \text { mit } \partial N_{v 1} / \partial q_{v 1}=N_{q_{v 1}}<0 \text { und } \partial N_{v 1} / \partial T_{i} & =N_{T}{ }^{\prime}<0, \\
i & =1,2, \ldots . . .
\end{aligned}
$$

Alle Verträge beginnen im Zeitpunkt $t=0$

Der Gewinn des Abbauunternehmers wird damit zu

$$
\mathbf{G}=\Sigma_{i}=1 \ldots N\left\{{ }_{0} T^{T}, N_{v i}\left(q_{v i}, T_{i}\right) q_{v i} e^{-\delta t d t}\right\}+{ }_{0} \int^{T}\left[p_{k}(t) q_{k}(t)-C(q(t))\right] e^{-\delta t d t},
$$

mit $q(t)=q_{k}(t)+\Sigma_{1}=j \ldots N\left(q_{v_{1}}\right)$ für $T_{1} \geq t$.

Der Abbauunternehmer löst das Optimierungsproblem

(6.51) $\max$

$E[U(G)]$

$\left(q_{v i}, q_{k}(t), T_{i}, T\right)$

u.d.B.

$R_{0} \geq \Sigma_{i}=1 \ldots N\left(q_{v i} T_{i}\right)+{ }_{0} \int^{T} q_{k}(t) d t, R_{0}$ gegeben,

$$
\begin{aligned}
q_{k}(t) & \geq 0 \text { für } t \in[0, T] \\
q_{v i} & \geq 0 \text { für } t \in\left[0, T_{1}\right] \\
q_{v i} & =0 \text { für } t \in\left(T_{1}, T\right] .
\end{aligned}
$$

und gelangt zu den notwendigen Bedingungen, $i=1,2, \ldots, N$

1) Bsp.: Die Situation eines erdölexportierenden Landes, das für die verschiedenen Abnehmer Auf-oder Abschläge vertraglich festschreibt und auf den Spotmärkten (Genua, Rotterdam, etc.) unter vollständiger Konkurrenz steht. 


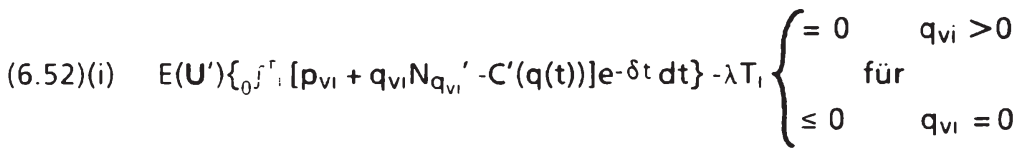

(6.52)(ii) $E\left\{U^{\prime}\left[p_{k}(t)-C^{\prime}(q(t))\right]\right\} e^{-\delta t}-\lambda$

$$
\left\{\begin{array}{l}
=0 \quad q_{k}(t)>0 \\
\leq 0 \quad \text { ür }
\end{array}\right.
$$

$$
\left\{\begin{array}{lll}
=0 & \lambda>0 \\
\text { für } & \lambda=0
\end{array}\right.
$$

(6.52)(iv) $E\left\{U^{\prime}\left\{q_{v i} N_{T_{1}{ }^{\prime}} \int^{T}{ }_{1} e^{-\delta t} d t+\left[p_{v i} q_{v i}+p_{k}\left(T_{i}\right) q_{k}\left(T_{i}\right)-C\left(q\left(T_{i}\right)\right)\right] e^{-\delta T_{1}}\right\}\right\}-$

$$
\lambda q\left(T_{i}\right)=0
$$

$(6.52)(v) \quad E\left\{U^{\prime}\left[p_{k}(T) q_{k}(T)-C\left(q_{k}(T)\right)\right]\right\} e^{-\delta T}-\lambda q_{k}(T)=0$.

Da Liefermenge und Lieferpreis für jeden Vertrag konstant sind, ist es für den Abbauunternehmer optimal, jene Rohstoffmenge über Vertrag zu verkaufen, bei der der in Erwartungsnutzeneinheiten bewertete Grenzgewinn über den Vertragszeitraum gleich dem mit der Laufzeit multiplizierten Gegenwartswert des Schattenpreises ist: 1$)$

$$
E\left(U^{\prime}\right)_{0} \int_{1}^{T}\left[p_{v_{1}}\left(1+n_{v_{1}}-1\right)-C^{\prime}(q(t))\right] e^{-\delta t} d t=\lambda T_{i}, \quad i=1,2, \ldots, N
$$

Im Optimum gilt zwischen den einzelnen Verträgen die Beziehung:

$$
\begin{aligned}
& T_{i}{ }^{-1}{ }_{0} \int_{1}^{T_{1}}\left[p_{v_{1}}\left(1+n_{v_{1}}{ }^{-1}\right)-C^{\prime}(q(t))\right] e^{-\delta t} d t= \\
& T_{J^{-1}} \int^{T}{ }_{1}\left[p_{v_{j}}\left(1+n_{v_{J}}{ }^{-1}\right)-C^{\prime}(q(t))\right] e^{-\delta t} d t, i \neq j ; i, j=1,2, \ldots, N
\end{aligned}
$$

Der ım (zeıtlichen) Durchschnitt über die Laufzeit erzielte Grenzgewinn aus den verschiedenen Verträgen ist gleich.

1) Dies folgt für $q_{v i}>0$ aus 6.52 (i). $n_{v i}=\left(\partial q_{v i} / \partial p_{v i}\right) p_{v i} q_{v i}-1$ steht für die Preiselastitzıtat der Nachfrage nach Vertragsabschlüssen der Art i. 
Weiter gilt zwischen jedem Vertrag $i$ und dem Kassaverkauf über dem Vertragszeıtraum T, die Gleichung 6.40, wonach der Barwert der Grenzerlöse aus dem Rohstoffverkauf über Vertrag i im Vertragszeitraum T, gleich ist dem Barwert der um eine Risikoprämie korrıgıerten Erwartungswerte der Kassapreise über diesen Zeitraum.

Die N Gleichungen in 6.52 (iv) machen Aussagen über Bedingungen, die in den Zeıtpunkten $T_{i}, i=1,2, \ldots, N$ gelten müssen. Für jedes i gilt eine Gleıchung der Art 6.43 und die dort gemachte Aussage.

Es gelte für die optimalen Vertragszeiträume der $N$ Verträge $T_{1}<T_{2}<\ldots$ $\ldots<T_{N}<T$. Schaubild 6.10 zeigt unter dieser Annahme das Abbauprofil und

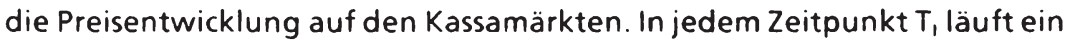
Liefervertrag aus. Die Abbaumenge sinkt in diesen Zeitpunkten um die jeweilige Periodenliefermenge. 
Schaubild 6.10: Die intertemporale Ressourcenallokation beı Lieferverträgen mit unterschiedlicher Laufzeit

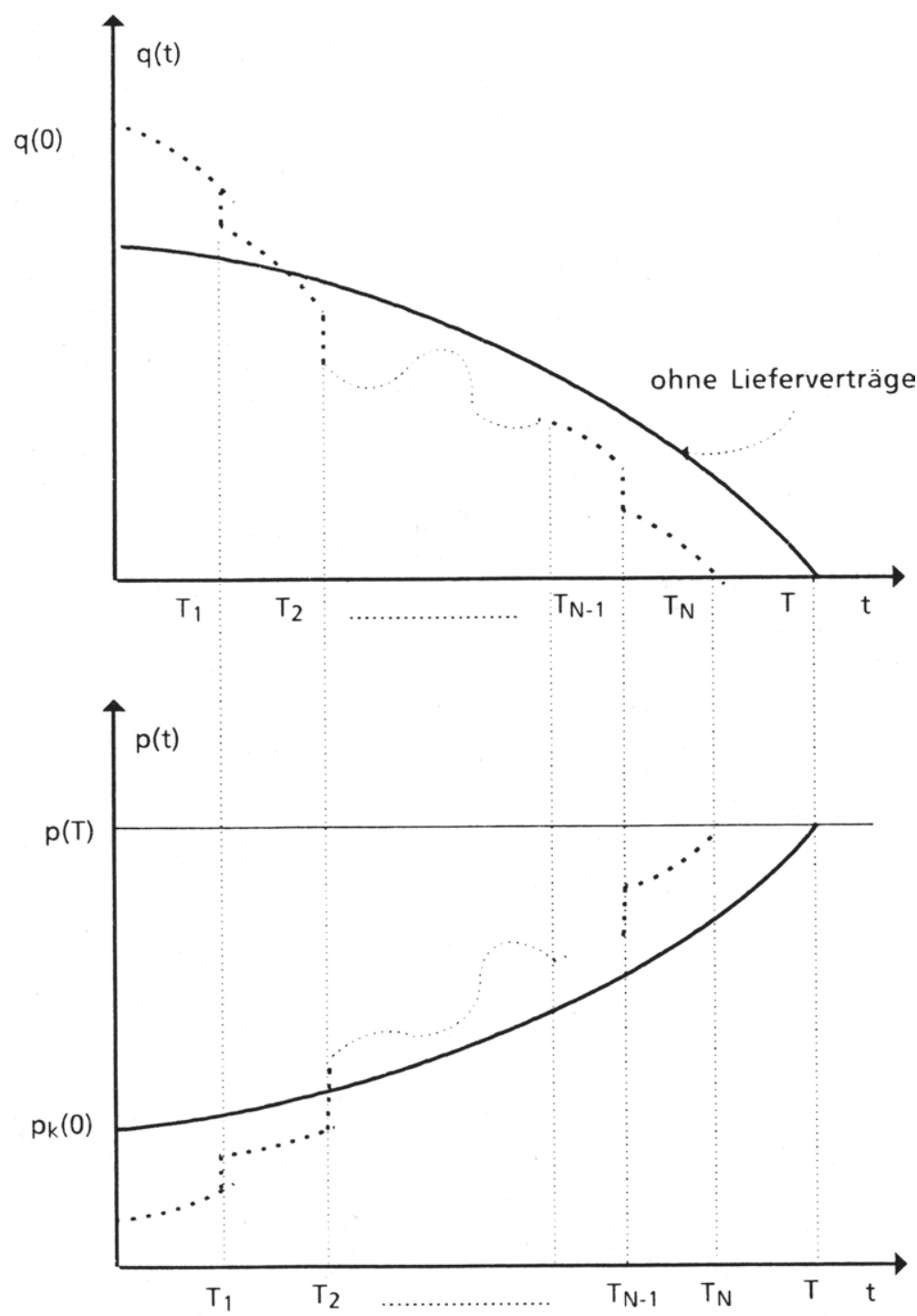




\subsubsection{Verträge mit variierenden Liefermengen und Liefer-} preisen

Nach der Verallgemeinerung auf $\mathbf{N}$ Verträge mit unterschiedlicher Laufzelt, werden nun die Annahmen einer im Vertragszeitraum konstanten Liefermenge und konstanter Lieferpreise aufgehoben.

Es gelten die Annahmen des Abschnitts 6.1.3.2. mit der nun lautenden Nachfrage nach Liefermengen über Vertragsabschlüsse

$$
\begin{aligned}
p_{v}(t)=N_{v}\left(q_{v}(t), T_{1}\right) \text { mit } \partial N_{v} / \partial q_{v} & =N_{q_{v}}{ }^{\prime}<0 \text { und } \\
\partial N_{v} / \partial T_{1} & =N_{T_{1}}{ }^{\prime}<0 .
\end{aligned}
$$

Als Gewinnfunktion ergibt sich

$$
G={ }_{0} \int_{2}\left[N_{v}\left(q_{v}, T_{1}\right) q_{v}(t)+p_{k}(t) q_{k}(t)-C(q(t))\right] e^{-\delta t d t}
$$

und aus dem Optimierungsansatz

$$
\begin{aligned}
& \max E[U(G)] \\
& \begin{array}{l}
\left(q_{v}, q_{k}(t), T_{1}, T_{2}\right) \\
\text { u.d.B. } \quad R_{0} \geq{ }_{0} \int_{2} q(t) d t, R_{0} \text { gegeben, } \\
\qquad q_{k}(t) \geq 0 \text { für } t \in\left[0, T_{2}\right], \\
q_{v}(t) \geq 0 \text { für } t \in\left[0, T_{1}\right], q_{v}(t)=0 \text { für } t \in\left(T_{1}, T_{2}\right], \\
p_{k}(t)=N\left(q_{k}(t)\right) u(t)
\end{array}
\end{aligned}
$$

folgt weiter

(6.57)(i) $E\left(U^{\prime}\right)\left[p_{v}(t)+q_{v}(t) N_{q_{v}^{\prime}} \cdot C^{\prime}(q(t))\right] e^{-\delta t}-\lambda T_{1} \quad\left\{\begin{array}{lll}=0 & & q_{v}(t)>0 \\ & \text { furr } & \\ \leq 0 & & q_{v}(t)=0\end{array}\right.$ 


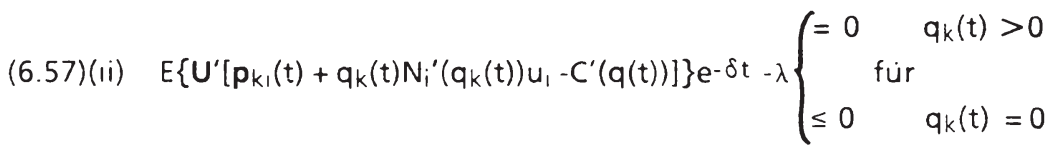

(6.57)(iii) $R_{0}-q_{v} T_{1}-{ }_{0} T_{2}^{T} q_{k}(t) d t$

$$
\left\{\begin{array}{lll}
=0 & \lambda>0 \\
\text { für } & \\
\geq 0 & \lambda=0
\end{array}\right.
$$

(6.57)(iv) $E\left\{U^{\prime}\left\{_{0} \int^{T} q_{v}(t) N_{T}, e^{-\delta t} d t+\right.\right.$

$$
\left.\left.\left[p_{v}\left(T_{1}\right) q_{v}\left(T_{1}\right)+p_{k 1}\left(T_{1}\right) q_{k}\left(T_{1}\right)-C\left(q\left(T_{1}\right)\right)\right] e^{-\delta T_{1}}\right\}\right\}-\lambda q\left(T_{1}\right)=0
$$

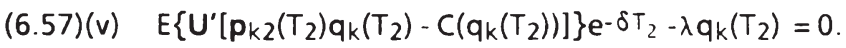

Im Optimum sind die Gegenwartswerte der Erwartungsnutzen der Grenzgewinne aus dem Kassaverkauf und dem Vertragsverkauf gleıch dem Schattenpreis der Ressource. Der Schattenpreis und damit die Erwartungsnutzen der Grenzgewinne wachsen bei optimalem Abbauprogramm mit der Diskontrate $\delta$.

(6.58)(i) $\quad E\left(U^{\prime}\right)\left[p_{v}(t)\left(1+\eta_{v^{-1}}\right)-C^{\prime}(q(t))\right]=\lambda e^{\delta t}$ und

(6.58)(ii) $E\left(U^{\prime}\right)\left\{\left\{E\left[p_{k_{1}}(t)\right]+\operatorname{cov}\left[U^{\prime}, p_{k_{1}}(t)\right] E\left(U^{\prime}\right)^{-1}\right\}\left(1+n_{k_{1}} e^{-1}\right)-C^{\prime}(q(t))\right\}=\lambda e^{\delta t}$, mit $i=1$ für $t \in\left[0, T_{1}\right]$ und $i=2$ für $t \in\left(T_{1}, T_{2}\right]$.

Hieraus folgt

$$
p_{v}(t)\left(1+n_{v^{-1}}\right)=\left\{E\left[p_{k 1}(t)\right]+\operatorname{cov}\left[U^{\prime}, p_{k 1}(t)\right] E\left(U^{\prime}\right)^{-1}\right\}\left(1+\eta_{k 1} e^{-1}\right)
$$

d.h. für jeden Zeitpunkt des Vertragszeitraumes gilt in laufenden Werten die Gleichheit zwischen dem Grenzerlös aus dem Vertragshandel und dem erwarteten Grenzerlös aus dem Kassahandel plus einem Abschlag für ein risikoaverses Abbauunternehmen. 
Die zeitliche Entwicklung der Vertrags- und Kassahandelsmengen läßt sıch aus 6.58 (i) bzw. (ii) ermitteln. Greıft man jedoch auf die Ergebnısse des Abschnittes 3.2.2. zurück, betrachtet diesen Fall als Preisdifferenzıerung unter Risiko, dann werden die Handelsprofile und das Abbauprofil direkt aus den entsprechenden Gleichungen erhalten.1)

Es folgt aus 3.32

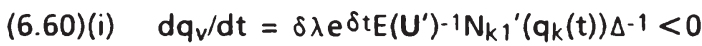

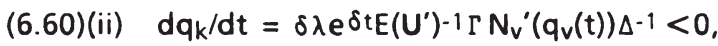

mit $\Delta=2 N_{v}{ }^{\prime}\left(q_{v}(t)\right) N_{k i}{ }^{\prime}\left(q_{k}(t)\right)+\left\{\lambda e^{\delta t E}\left(U^{\prime}\right)-2\left[d E\left(U^{\prime}\right) / d q_{k}\right]-C^{\prime \prime}(q(t))\right\}$

$$
\left[N_{k}{ }^{\prime}\left(q_{k}(t)\right)+\Gamma N_{v}{ }^{\prime}\left(q_{v}(t)\right)\right]>0, i=1,2 .
$$

Ein Vergleich der Handelsprofile ergibt

$$
d q_{v} / d t>_{<} d q_{k} / d t \Leftrightarrow N_{k} 1^{\prime}\left(q_{k}(t)\right)>_{<\Gamma} N_{v}{ }^{\prime}\left(q_{v}(t)\right) .
$$

Bei identischem Nachfrageverlauf für Kassa- und Vertragslieferungen führt das Risikoverhalten gegenüber dem Kassamarkt zu abweichenden Handelsprofilen:

$\left(6.61^{\prime}\right) \quad d q_{v} / d t><d q_{k} / d t \Leftrightarrow \Gamma><1$.

Ein risikoaverser Abbauunternehmer $(r>1)$ bietet auf dem Kassamarkt mit größeren zeitlichen Veränderungen seiner Handelsmengen an als auf dem Vertragsmarkt. Er bleibt länger am Vertragsmarkt. Ein risikofreudiger $A b$ bauunternehmer $(\Gamma<1)$ hingegen wählt die umgekehrte Relation der zeitlichen Entwicklung der Handelsprofile, und ein risikoneutraler Abbauunternehmer ( $\Gamma=1$ ) wählt für beide Märkte das gleiche Handelsprofil.

1) Man setze $N_{1}{ }^{\prime}\left(q_{1}(t)\right)=N_{v}{ }^{\prime}\left(q_{v}(t)\right), N_{2}{ }^{\prime}\left(q_{2}(t)\right)=N_{k 1}{ }^{\prime}\left(q_{k}(t)\right)$ und beachte, daß dann gilt $\Gamma_{1}=1$ und $\Gamma_{2}=\Gamma$. 
Weist der Vertragsmarkt einen unelastischeren Nachfrageverlauf auf $\left(N_{k} i^{\prime}\left(q_{k}(t)\right)>N_{v}{ }^{\prime}\left(q_{v}(t)\right)\right)$, so wahlt der risikoaverse Abbauunternehmer für den Vertragshandel ımmer eın flacheres Handelsprofil als für den Kassahandel. Gilt dagegen die umgekehrte Relation $\left(N_{k_{i}}{ }^{\prime}\left(q_{k}(t)\right)<N_{v}{ }^{\prime}\left(q_{v}(t)\right)\right)$, so Ist eıne eındeutige Aussage uber das Verhältnis der Handelsprofile nicht möglich. Je nach Ausmaß der Risıkoaversıon können die Handelsprofile gleich oder unterschiedlich sein.

Das Abbauprofil ergibt sich aus $6.60 \mathrm{mit} \mathrm{dq} / \mathrm{dt}=\mathrm{dq} \mathrm{q}_{\mathrm{v}} / \mathrm{dt}+\mathrm{dq} \mathrm{q}_{\mathrm{k}} / \mathrm{dt} \mathrm{zu}$

$$
d q / d t=\delta \lambda e^{\delta t E}\left(U^{\prime}\right)^{-1}\left[N_{k_{1}}{ }^{\prime}\left(q_{k}(t)\right)+\Gamma N_{v}{ }^{\prime}\left(q_{v}(t)\right)\right] \Delta^{-1}<0
$$

Bei Vertragsabschlüssen mit konstanten Liefermengen im Vertragszeitraum baut der Monopolist ab gemaß1)

$$
\begin{aligned}
\mathrm{dq} / \mathrm{dt}= & \delta \lambda e^{\delta t E\left(U^{\prime}\right)-1} \\
& \left\{2 N_{k} 1^{\prime}\left(q_{k}(t)\right) \Gamma^{-1}-C^{\prime \prime}(q(t))+\lambda e^{\delta t E}\left(U^{\prime}\right)-2\left[d E\left(U^{\prime}\right) / d q_{k}\right]\right\}-1,
\end{aligned}
$$

mit

$$
N_{1}{ }^{\prime}\left(q_{k}(t)\right)=\left\{\begin{array}{l}
N_{1}{ }^{\prime}\left(q_{k}(t)\right) \text { im Vertragszeitraum } \\
N_{2}{ }^{\prime}\left(q_{k}(t)\right) \text { außerhalb des Vertragszeitraumes }
\end{array}\right.
$$

und $N_{1}{ }^{\prime}\left(q_{k}(t)\right)>N_{2}{ }^{\prime}\left(q_{k}(t)\right)$.

Sei für Vergleichszwecke wieder $E\left(U^{\prime}\right)^{-1}\left[d E\left(U^{\prime}\right) / d q_{k}\right]$ als vernachlässigbar klein unterstellt. Weiter ist für eine eindeutige Aussage eine einschränkende Annahme über die Abbaukosten erforderlich. Es seien die durchschnittlichen Abbaukosten konstant. Der Index fbzw. c bezeichne die Abbauprofile für variierende und fixe Liefermengen.

1) Siehe hierzu Abschnitt 6.1.3.2. 
6.62 und 6.63 werden dann zu

$$
\begin{aligned}
&(d q / d t)_{f}=\delta \lambda_{f} e^{\delta t E}\left(U^{\prime}\right)^{-1}[\left.N_{k} 1^{\prime}\left(q_{k}(t)\right)+\Gamma N_{v}{ }^{\prime}\left(q_{v}(t)\right)\right] \\
&\left\{2 N_{k} 1^{\prime}\left(q_{k}(t)\right) N_{v}\left(q_{v}(t)\right)\right\}^{-1}<0,
\end{aligned}
$$

$$
(d q / d t)_{C}=\delta \lambda_{c} e^{\delta t E}\left(U^{\prime}\right)^{-1} \Gamma\left[2 N_{1}{ }^{\prime}\left(q_{k}(t)\right)\right]^{-1}<0 .
$$

Im Vertragszeitraum gelte für beide Arten vertraglicher Ausgestaltung der gleiche Nachfrageverlauf des Kassamarktes $N_{k} 1^{\prime}\left(q_{k}(t)\right)=N_{1}{ }^{\prime}\left(q_{k}(t)\right)$.

Hieraus folgt

$$
\begin{aligned}
& (d q / d t)_{c}>(d q / d t)_{f} \Leftrightarrow \delta \lambda_{c} e^{\delta t E}\left(U^{\prime}\right)^{-1} \Gamma\left[2 N_{1}\left(q_{k}(t)\right)\right]^{-1}> \\
& \delta \lambda_{f} e^{\delta t E}\left(U^{\prime}\right)^{-1}\left[N_{1}^{\prime}\left(q_{k}(t)\right)+\Gamma N_{v}\left(q_{v}(t)\right)\right]\left\{2 N_{1}^{\prime}\left(q_{k}(t)\right) N_{v}\left(q_{v}(t)\right)\right\}^{-1} .
\end{aligned}
$$

Es sei weiter unterstellt die annähernde Gleichheit der Gegenwartswerte der Grenzgewinne aus dem Vertragsverkauf bei konstanten und variablen Liefermengen:

$$
\begin{aligned}
& T_{1} 1^{-1}{ }_{0} \int^{T} 1\left[p_{v}+q_{v} N_{v}{ }^{\prime}\left(q_{v}\right)-c\right] e^{-\delta t} d t \approx\left[p_{v}(t)+q_{v}(t) N_{v}{ }^{\prime}\left(q_{v}(t)\right)-c\right] e^{-\delta t} \\
& \Leftrightarrow \quad \lambda_{c} E\left(U^{\prime}\right)-1 \approx \lambda_{f} E\left(U^{\prime}\right)^{-1} . \\
& \Rightarrow \quad(d q / d t)_{c}>(d q / d t)_{f} \Leftrightarrow \Gamma\left[2 N_{1}{ }^{\prime}\left(q_{k}(t)\right)\right]^{-1}> \\
& {\left[N_{1}{ }^{\prime}\left(q_{k}(t)\right)+\left[N_{v}{ }^{\prime}\left(q_{v}(t)\right)\right]\left\{2 N_{1}{ }^{\prime}\left(q_{k}(t)\right) N_{v}{ }^{\prime}\left(q_{v}(t)\right)\right\}-1\right.} \\
& \Leftrightarrow 0>N_{1}{ }^{\prime}\left(q_{k}(t)\right) \text {. }
\end{aligned}
$$

Im Vertragszeitraum führen variable Liefermengen zu einem schnelleren Abbau, da nun neben den Kassa- auch die Vertragshandelsmengen abnehmen. Geht man von einer gesamten kontraktierten Rohstoffmenge aus, die über die Vertragszeiträume für beide Vertragsarten gleich sein soll, so wird bei variablen Liefermengen der Vertragshandel schneller zur Erfüllung kommen. Dies führt dann weiter dazu, daß auch der restliche Ressourcenbestand zu einem früheren Zeitpunkt als im Falle fixer Vertragsmengen erschöpft wird. 


\subsection{Vertragsvereinbarungen auf dem Reservenmarkt}

Die Analyse der Auswirkungen von Vertragsvereınbarungen auf die intertemporale Allokation erschöpfbarer Naturressourcen steckt im Vergleich mit der Analyse anderer Bestimmungsfaktoren noch in den "Kinderschuhen".

Bei Roberts (1980) findet man eıne Analyse zu den Allokationswirkungen von langfristigen Lieferverträgen zwischen Abbauunternehmen und Unternehmen, die Rohstoffe verarbeiten. Von Sutinen (1980) wird in einem kurzen Beitrag der Einfluß von Vereinbarungen zwischen Reservenbesitzern und Abbauunternehmern über den Reservenpreis (Förderabgabe) auf die Ressourcenallokation diskutiert. Mit diesen beiden Beiträgen, der Diskussion auf dem Rohstoffmarkt und auf dem Reservenmarkt, erschöpft sich die Literatur zu den Allokatıonswirkungen von Vertragsvereinbarungen.

Die Absicht dieses Abschnittes ist es, die Analyse der Allokationswirkungen von Vereinbarungen zwischen Reservenbesitzer und Abbauunternehmern in einen weiteren Rahmen einzuordnen als es bei Sutinen geschehen ist und die Diskussion intensiver und extensiver zu gestalten.

Diesem Abschnitt liegt das in Abschnitt 2.4. eingeführte zweistufige Marktkonzept aus Rohstoff- und Reservenmarkt zugrunde. Rısiko wird wieder auf dem Rohstoffmarkt über die Nachfrage eingeführt. Für verschiedene Formen der Vereinbarungen auf dem Reservenmarkt zwischen dem Abbauunternehmen und dem Reservenbesitzer werden die Allokationsergebnisse diskutiert, und zwar die drei Vereinbarungsarten der Konzession ( $A$ bschnitt 6.2.1.), der Abbaumengenteilung (Abschnitt 6.2.2.) und des Dienstleistungsvertrages (Abschnitt 6.2.3.) sind Gegenstand der Modellbildung. 


\subsubsection{Konzessionsmodelle}

Gegenüber Abschnitt 2.4. ändern sich die Annahmen wie folgt: 1)

Auf dem Rohstoffmarkt seien die Verarbeitungsunternehmen repräsentiert durch die Nachfragefunktion

$$
p(t)=N(q(t)) u(t) \quad \operatorname{mit} N^{\prime}(q(t))<0, N^{\prime \prime}(q(t))=0 .
$$

$u(t)$ steht wieder für eine nichtnegative Zufallsvariable, die identisch und unabhängig über die Zeit verteilt ist. Für ihren Erwartungswert und ihre Varianz gelte: $E[u(t)]=1$ und $\operatorname{var}[u(t)]=\sigma^{2}$.

$p(t)$ und $q(t)$ bezeichnen den Preis und die Menge des Rohstoffes.

Für eine gegebene Folge von Preisen $\{r(t)\}$ für Abbaurechte und einer bestimmten Erwartungshaltung gegenüber den Preisrealisationen auf dem Rohstoffmarkt $\{E[p(t)]\}$, wählen die Abbauunternehmen ihre Nachfrage nach Abbaurechten und ihr Angebot an Rohstoffen so, daß sie den Erwartungswert der Gesamtheit $\mathbf{G}$ der diskontierten Periodengewinne maximieren:

$$
G={ }_{0} \int^{T}[p(t) q(t)-c q(t)-r(t) q(t)] e-\delta t d t .
$$

Jeder Abbauunternehmer besitze die Nutzenfunktion $\left.U_{A}(G) 2\right)$ mit $U_{A^{\prime}}>0$ und $U_{A} "<0$, d.h. für die Abbauunternehmer wird Risikoaversion unterstellt.

1) Zu den Konzessionsmodellen dieses Abschnittes vgl. Sutinen (1980), S.171-176.

2) Im Unterschied zu Sutinen wird keine Periodennutzenfunktion unterstellt. Die hier verwendete Nutzenfunktion ist zu verstehen als eine Risikopräferenzfunktion, wie sie von Krelle (1968), S.140-147 für den statischen Fall und von Sinn (1980), S.244f für den dynamischen Fall als Ergebnis einer Kritik an der Modellierung von Risikofurcht durch Periodennutzenfunktionen eingeführt wird. 
Die Reservenbesitzer seien ebenfalls als risikoavers angenommen. Es gelte für jeden Reservenbesitzer die Nutzenfunktion $U_{R}(E) m_{1} U_{R}{ }^{\prime}>0$ und $U_{R}{ }^{\prime \prime}<0$. E bezeıchne die Gesamtheit der diskontierten Einnahmen aus dem Verkauf von Abbaurechten uber einen bekannten Ressourcenbestand $R_{0}$. Dıe Gesamtheit der Lagerstatten sei bekannt und die Qualıtät der Rohstoffe in den Lagerstätten gleıch.

Neben dem Reserven- und Rohstoffmarkt exıstiere ein vollkommener Kapitalmarkt, auf dem Gewinne mit der risikolosen Zinsrate $\delta$ angelegt werdenkönnen.

\subsubsection{Deterministischer Reservenpreis bzw. mengenbezogene} Förderabgabe

Bei einer Konzession zahlt das Abbauunternehmen einen bestimmten, bekannten Preis $r(t)$ für das Recht, Rohstoffe abbauen zu dürfen. Die Abbaurechte werden zwischen den Reservenbesitzern und den Abbauunternehmen auf dem Reservenmarkt gehandelt. Zu einer gegebenen Folge von Preisen $\{r(t)\}$ wählen Reservenbesitzer bzw. Abbauunternehmen das Angebot bzw. die Nachfrage nach Abbaurechten.

Für die Reservenbesitzer gilt dieselbe Problemstellung wie in 2.55 :

$$
\begin{aligned}
& \max { }_{0} \int^{\top} r(t) q(t) e^{-\delta t} d t \\
& (q(t)) \\
& \text { u.d.B. } R_{0} \geq{ }_{0} S^{\top} q(t) d t, R_{0} \text { gegeben, } q(t) \geq 0
\end{aligned}
$$

Man erhält an notwendigen Bedingungen 
(6.67)(i) $r(t) e^{-\delta t-\lambda}$

$$
\begin{aligned}
& \left\{\begin{array}{lr}
=0 & q(t)>0 \\
& \text { für } \\
\leq 0 & q(t)=0
\end{array}\right. \\
& \left\{\begin{array}{lr}
=0 & \lambda>0 \\
& \text { für } \quad \\
\geq 0 & \lambda=0
\end{array}\right.
\end{aligned}
$$

Es folgt $r(t)=\lambda e^{\delta t}$ bzw. $r(t)=r(0) e^{\delta t}$.

Hiermit hat man die Angebotsfunktion für den Reservenmarkt. Die Reservenbesitzer sind zu einem Angebot an Abbaurechten dann bereit, wenn der Preis für Abbaurechte mit der Diskontrate $\delta$ wächst. Haben die Reservenbesitzer ihre Gewinnmaxima für ein $r(0)$ realisiert, dann gilt $R_{0}={ }_{0} S^{\top} q(t) d t$, d.h. der gesamte Reservenbestand ist über Abbaurechte an die Abbauunternehmer verkauft.

Einem Abbauunternehmer stellt sich das Optimierungsproblemi)

(6.68) $\max E[U(G)]$ u.d.B. $q(t) \geq 0$.

$(q(t))$

Die notwendige Bedingung lautet

1) Mit G $={ }_{0} T^{T}[p(t)-c-r(t)] q(t) e^{-\delta} \delta t d$. 


$$
E\left\{U^{\prime}{ }_{A}[p(t)-c-r(t)]\right\} e^{-\delta t-\lambda} \quad\left\{\begin{array}{cc}
=0 & q(t)>0 \\
& \text { für } \\
\leq 0 & q(t)=0 .
\end{array}\right.
$$

Fur $q(t)>0$ erhält man

$$
E\left[U^{\prime}{ }_{A} p(t)\right]=E\left(U_{A}^{\prime}\right)[c+r(t)]
$$

bzW. mit $E\left[U_{A}^{\prime} P(t)\right]=E\left(U_{A}^{\prime}\right) E[p(t)]+\operatorname{cov}\left[U^{\prime}{ }_{A, p}(t)\right]$

$$
E[p(t)]+\operatorname{cov}\left[U_{A}^{\prime}, p(t)\right] E\left(U_{A}^{\prime}\right)^{-1}-c=r(t)
$$

Ein Abbauunternehmer frägt jene Menge an Abbaurechten für die Periode t nach, bei der der Preis für das Abbaurecht gleich dem um eine Risikorate korrigierten, erwarteten Grenzgewinn dieser Periode ist.

Da für eınen risikoaversen Abbauunternehmer $\operatorname{cov}\left[U^{\prime}{ }_{A}, P(t)\right]<0$ gilt, folgt aus einem Vergleich c.p. mit einem risikoneutralen Abbauunternehmer, daß Risikoaversion die Nachfrage nach Abbaurechten mindert.

An dieser Stelle bietet sich wieder die Definition einer Variablen $\Gamma_{i}$ an mit

$$
\begin{aligned}
& \Gamma_{1}=E[p(t)]\left\{E[p(t)]+\operatorname{cov}\left[U^{\prime}{ }_{i}, p(t)\right] E\left(U^{\prime}{ }_{i}\right)^{-1}\right\}-1, i=A, R \\
& \text { und } \Gamma_{1}>1 \text { bei Risikoaversion. }
\end{aligned}
$$

Gleichung 6.70 vereinfacht sich damit zu

$$
E[p(t)]=\Gamma_{A}[c+r(t)]
$$

$\Gamma_{A}$ ist wie zuvor zu interpretieren als ein durch Risikoverhalten induzierter Bias im Angebots- bzw. Nachfrageverhalten eines Abbauunternehmers.

Die Nachfrage nach Abbaurechten ist eine abgeleitete Nachfrage aus der Nachfrage nach Rohstoffen, für die unterstellt wurde $p(t)=N(q(t)) u(t)$. Da $E[u(t)]=1$, gilt $E[p(t)]=N(q(t))$. 
Unter der Annahme rationaler Erwartungen der Abbauunternehmen über die gleıchgewichtigen Preise des Rohstoffmarktes folgt fur die Nachfrage nach Abbaurechten

$$
r(t)=N(q(t)) \Gamma_{A^{-1}-c}
$$

Die Differentiation nach der Zeit liefert

$$
d r / d t=\left[\Gamma_{A} N^{\prime}(q(t))(d q / d t)-N(q(t))\left(d \Gamma_{A} / d t\right)\right] \Gamma_{A^{-2}}
$$

Berücksichtigt man hier die Bedingung $r(t)=r(0)$ e $\delta t$, die erforderlich ist, damit die Reservenbesitzer zu jedem Zeitpunkt zu einem positiven Angebot bereit sind, so erhält man für die zeitliche Entwicklung der gehandelten Abbaurechte1)

$$
d q / d t=\delta r(t) \Gamma_{A} N^{\prime}(q(t))-1<0 .
$$

Mit Gleichung 6.72 wird aus 6.74 erhalten2)

$$
\left.d q / d t=\delta\left[N(q(t))-c \Gamma_{A}\right] N^{\prime}(q(t))^{-1}<0.3\right)
$$

Für gegebene Erwartungen über die Preise auf den zukünftigen Kassamärkten, $E[p(t)]=N(q(t))$, erhält man in Abhängigkeit vom Risikoverhalten

1) Vgl. Anhang III. Es ist ein konstantes Risiko bzw. eine konstante Risikoaversion unterstellt.

2) Zu diesem Ergebnis vgl. Sutinen (1980), S.172. Sutinen gibt seine Ergebnisse in Wachstumsraten an und vergleicht diese. Da sich sowohl $\mathrm{dq} / \mathrm{dt}$ als auch $\mathrm{q}(\mathrm{t})$ beim Übergang zu anderem Risikoverhalten in die

3) gleiche Richtung ändern, sind seine Ergebnisse nicht schlüssig.

3) Zu diesem Resultat führt auch die Annahme einer von den Abbaukosten abhängigen Vergütung des-Abbaurechtes:

Sei $r(t)=\theta(t) c$, dann erhält man als notwendige Bedingung für den Reservenbesitzer $\theta(t) c=\lambda e \delta t, q(t)>0$ und für das $A b b a u$ buternehmen $E\left[U_{A}^{\prime} p(t)\right]=E\left(U_{A^{\prime}}\right)[1+\theta(t)] c$. Im Marktgleichgewicht gilt:

$N(q(t))=\Gamma_{A} c+\lambda e \delta t$, woraus folgt $d q / d t=\delta N^{\prime}(q(t))-1\left[N(q(t))-\Gamma_{A} c\right]$. 
die Aussage, daß im Falle eınes risikoaversen Abbauunternehmers $\left(\Gamma_{A}>1\right)$ die Fordermengen mit kleıneren Veranderungen abnehmen als bei einem risikoneutralen $\left(\Gamma_{A}=1\right)$ oder risıkofreudigen Abbauunternehmer $\left(\Gamma_{A}<1\right)$. Das bedeutet, Verlagerung des Abbaus In die Zukunft. Damit hat man die Ergebnisse, wie sie auch aus dem eınstufigen Marktkonzept in Abschnitt 3.1. erhalten wurden und in Schaubild 3.1 dargestellt sind.

Darüber hinaus sollen in diesem Modellzusammenhang auch Aussagen gemacht werden über die Auswirkungen von Risikoänderungen. Eine Änderung des Risikos auf dem Rohstoffmarkt1) oder, was diesem gleichkommt, eine Änderung des Verhaltens gegenüber Risiko, führt zu den folgenden Änderungen bei den erwartungsnutzenoptimalen Fördermengen $q(t)$ und den gleichgewichtigen Preisen $r(t)$ des Marktes für Abbaurechte: 2 )

$$
(d r / d t)-N^{\prime}(q(t)) \Gamma_{A^{-1}}\left(d q / d \Gamma_{A}\right)=-N(q(t)) \Gamma_{A^{-2}}<0 .
$$

Interpretiert man 6.75 als Gleichgewichtsbedingung des Rohstoffmarktes bei gegebenem $r(t)$, dann folgt

$$
\left(d q / d \Gamma_{A}\right) d r=0=N(q(t))\left[N^{\prime}(q(t)) \Gamma_{A}\right]^{-1}<0 .
$$

Wird Gleichung 6.75 als Gleichgewichtsbedingung des Marktes für Abbaurechte bei gegebener Rohstoffnachfrage $q(t)$ betrachtet, so führt dies zu

$\left(6.76^{\prime \prime}\right) \quad\left(d r / d \Gamma_{A}\right)_{d q}=0=-N(q(t)) \Gamma_{A}-2<0$.

Als Auswirkungen auf den Abbauzeitraum folgt über die notwendige Bedingung 6.67 (ii) für $\lambda>0$ :

$$
d q(t)=-q(T) d T \text { und weiter }
$$

$$
d q / d \Gamma_{A}=-q(T)\left(d T / d \Gamma_{A}\right) \text {. }
$$

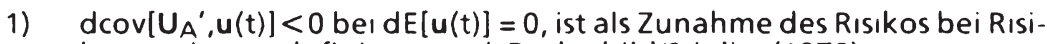
koaversion zu definieren; vgl. Rothschild/Stiglitz (1970).

2) $d \Gamma_{A}>0 \Leftrightarrow \operatorname{dcov}\left[U_{A^{\prime}}, u(t)\right]<0$ für $d E[u(t)]=0$. Gleichung 6.76 folgt aus 6.72 . 
Damit ist

(6.77') $d T / d \Gamma_{A}><0 \Leftrightarrow d q / d \Gamma_{A}><0$.

Die Zunahme des Risikos auf dem Rohstoffmarkt läßt die Handelsmengen des Rohstoffmarktes sinken. Als Folge davon sınken die gehandelten Mengen an Abbaurechten. Aufgrund des starren Angebots an Abbaurechten sinkt deren Preis ebenfalls. Der Abbauzeitraum vergroßert sich.

Neben dem Niveau der Handelsmengen wird sich auch deren zeitliche Entwicklung verändern. Gleichung 6.75 liefert:

$$
\begin{aligned}
(d q / d t) d \Gamma_{A}=\delta N^{\prime}(q(t))-2 . & \\
& \left\{-c N^{\prime}(q(t))+\left\{N^{\prime}(q(t))^{2}-\left[N(q(t))-c \Gamma_{A}\right] N^{\prime \prime}(q(t))\right\}\left(d q / d \Gamma_{A}\right)\right\}
\end{aligned}
$$

und weiter mit $6.76^{\prime}$

$$
(d q / d t) d \Gamma_{A}=\delta\left[N(q(t))-c \Gamma_{A}\right]\left[N^{\prime}(q(t))^{2}-N(q(t)) N^{\prime \prime}(q(t))\right] N^{\prime}(q(t))-2 .
$$

Nach 6.72 gilt $N(q(t))-c \Gamma_{A}>0$ und man erhält:

$$
(d q / d t) d \Gamma_{A}>_{<0} \Leftrightarrow N^{\prime}(q(t))>_{<N}(q(t)) N^{\prime \prime}(q(t)) .
$$

Im Falle einer linearen $\left(N^{\prime \prime}(q(t))=0\right)$ oder einer konvexen Nachfrage $\left(N^{\prime \prime}(q(t))<0\right)$ auf dem Rohstoffmarkt erhält man das Ergebnis, daß der Abbaupfad eines risikoaversen Förderunternehmens als Reaktion auf eine Risikoerhöhung einen flacheren Verlauf aufweisen wird. Der risikoaverse $A b$ bauunternehmer verschiebt seinen Abbau in die Zukunft, um sein Risiko durch "intertemporale Diversifikation" zu mindern.

Liegt eine konkave Nachfrage $\left(N^{\prime \prime}(q(t))>0\right)$ auf dem Rohstoffmarkt vor, so kommt man zu der Aussage:

Je (un-)elastischer der systematische Teil der Nachfrage des Rohstoffmarktes ist, desto eher ist zu erwarten, daß die Abbauunternehmen mit einem (langsameren) schnelleren Abbau auf eıne Zunahme des Risikos reagieren werden. 
Neben dieser komparativen Betrachtung für unterschıedliche Rısiken bietet es sich an, auch die intertemporale Allokatıon für ein im Zeıtablauf steıgen-

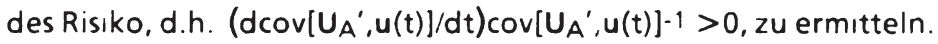

Aus Gleichung 6.73 erhalt man

$$
d q / d t=\delta\left[N(q(t))-c \Gamma_{A}\right] N^{\prime}(q(t))^{-1}+N(q(t)) N^{\prime}(q(t))^{-1}\left[\left(d \Gamma_{A} / d t\right) \Gamma_{A^{-1}}\right] .
$$

Mit

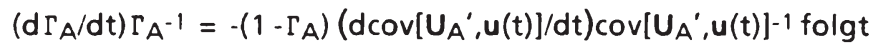

$$
\begin{aligned}
d q / d t= & \delta\left[N(q(t))-c \Gamma_{A}\right] N^{\prime}(q(t))^{-1}- \\
& \left.N(q(t)) N^{\prime}(q(t))\right)^{-1}\left(1-\Gamma_{A}\right)\left(d \operatorname { c o v } \left[U _ { A ^ { \prime } , u ( t ) ] / d t ) } \operatorname { c o v } \left[U_{\left.A^{\prime}, u(t)\right]^{-1} .}\right.\right.\right.
\end{aligned}
$$

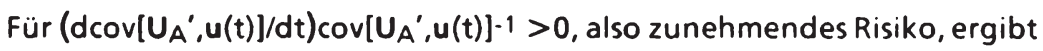
sich das Allokationsergebnis

(6.83)(i) $\quad(d q / d t)_{d} \Gamma_{A} / d t>0<(d q / d t) d \Gamma_{A} / d t=0<0$ und

(6.83)(ii) $\quad(d p / d t)_{d} \Gamma_{A} / d t=0>(d p / d t) d \Gamma_{A} / d t>0>0$.

Nimmt das Risiko im Zeitablauf zu, dann führt dies bei einem risikoaversen Abbauunternehmen im Vergleich zum Fall konstanten Risikos zu einem schnelleren Abbau des Bestandes. Denn durch die Umverteilung zugunsten der Gegenwart kann das Risiko gemindert werden.

Schaubild 6.11 stellt diese Allokationen einander gegenüber. Hinzugefügt sind die Pfade für abnehmendes Risiko. 
Schaubild 6.11: Die intertemporale Ressourcenallokatıon für zeitlich varuerendes Risiko

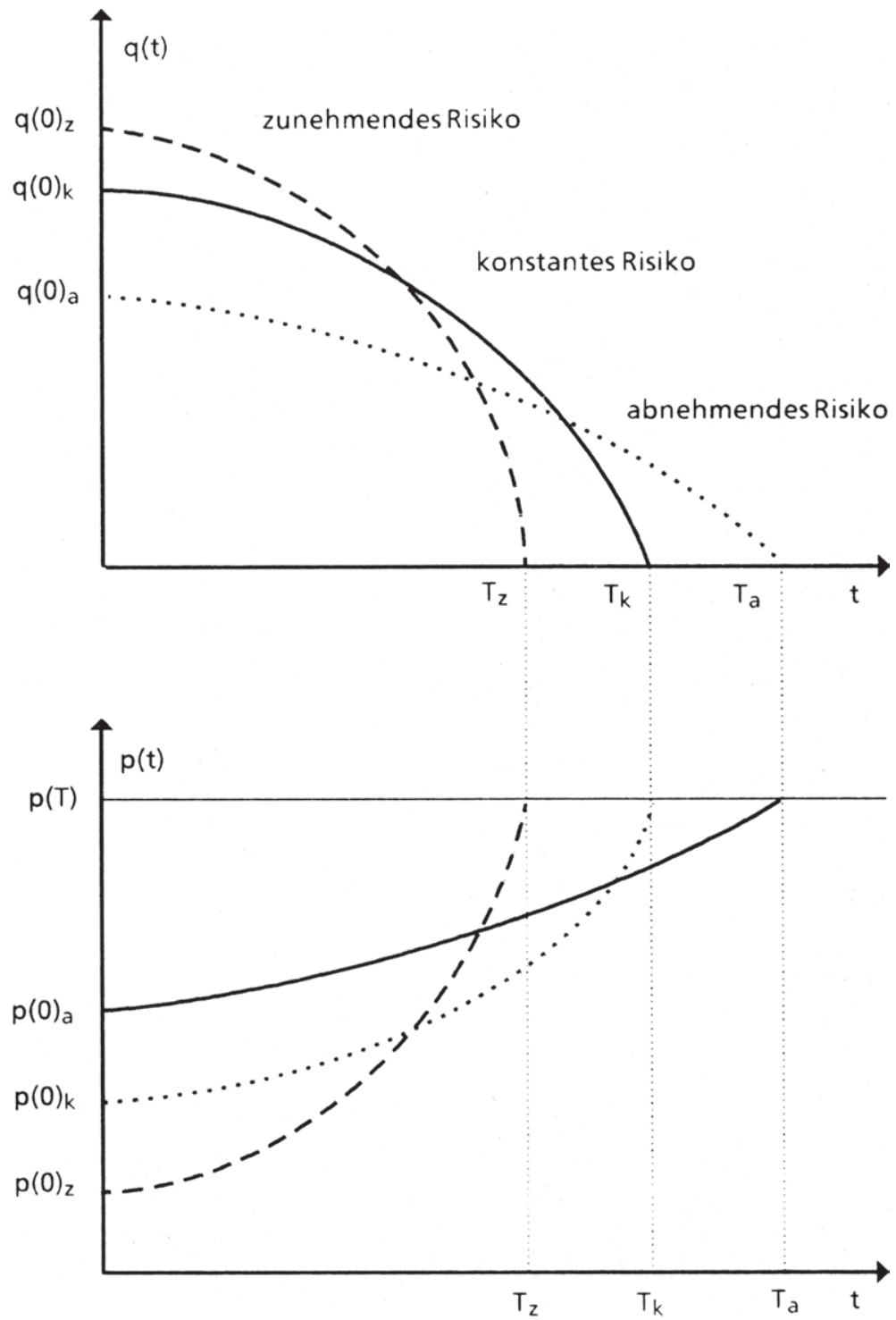




\subsubsection{Indexierter Reservenpreis bzw. mengen- und wertbezo- gene Förderabgabe}

Im Modell zuvor wurde den Reservenbesitzern das Abbaurecht mit einem Preis entgolten, der unabhängig von der Situation des Rohstoffmarktes war. Das Marktrisiko trugen die Abbauunternehmen. Nun soll der Preis für das Abbaurecht an den Rohstoffpreis gebunden sein. Das Preisrısiko auf dem Rohstoffmarkt verteilt sich auf Reservenbesitzer und Abbauunternehmer. Ein Preisanstieg auf dem Rohstoffmarkt führt für den Abbauunternehmer zu einem Preisanstieg für das Abbaurecht.

Es soll gelten 1)

$$
r(t)=\alpha(t)+\beta(t) p(t) \text { mit } 0 \leq \beta(t) \leq 1 .
$$

Für $\beta(t)=0$ hat man den vorigen Fall einer mengenbezogenen Förderabgabe bzw. eines deterministischen Reservenpreises. Ein $B(t)=0$ wird zu erwarten sein, wenn $B(t)$ als ein auszuhandelnder Parameter zwischen risikoaversen Reservenbesitzern und risikoneutralen Abbauunternehmern betrachtet wird.

Ein $B(t) \rightarrow 1$ ist zu erwarten, wenn das umgekehrte Verhalten gegenüber $R i-$ siko unterstellt wird.2)

Sind sowohl die Abbauunternehmer als auch die Reservenbesitzer risikoavers, dann werden sie ein $B(t)$ wählen, für das gilt $0<B(t)<1$.3) Zunächst soll von gegebenen Werten $\alpha(t)$ und $B(t)$ ausgegangen werden.

1) Vgl. Sutinen (1980), S.173. Dort wird dieser Ansatz als "risk sharing lease" bezeichnet

2) Vgl. Anhang VIII.

3) Dieskann gezeigt werden mittels eines Ansatzes, wie er mit $6.94 \mathrm{er}$ folgt. Vgl. Anhang VII. 
In diesem geanderten Modellzusammenhang maximieren die Reservenbesitzer nun ebenfalls den Erwartungsnutzen über ihren gesamten Einnahmen:

$$
\begin{aligned}
& \max _{(q(t))} E\left\{U\left[{ }_{0} S^{\top}[\alpha(t)+B(t) p(t)] q(t) e^{-} \delta t d t\right\}\right. \\
& \text { u.d.B. } \quad R_{0} \geq{ }_{0} \int^{\top} q(t) d t, R_{0} \text { gegeben, } q(t) \geq 0 .
\end{aligned}
$$

An notwendigen Bedingungen werden erhalten

$$
E\left\{U_{R}^{\prime}[\alpha(t)+\beta(t) p(t)]\right\} e^{-\delta t}-\lambda
$$

(6.85)(ii) $R_{0}-{ }_{0} \int^{\top} q(t) d t$

$$
\begin{aligned}
& \begin{cases}=0 & q(t)>0 \\
& \text { für } \\
\leq 0 & q(t)=0\end{cases} \\
& \begin{cases}=0 & \lambda>0 \\
\text { für } & \\
\geq 0 & \lambda=0 .\end{cases}
\end{aligned}
$$

Damit gilt für positive $q(t)$

$$
\left.E\left(U_{R}^{\prime}\right) \alpha(t)+B(t) E\left[U_{R}^{\prime} p(t)\right]\right\} e^{-\delta t}=\lambda e^{\delta t} .
$$

Das Angebot an Abbaurechten hängt nun direkt ab vom Preis des Rohstoffmarktes bzw. von Erwartungen über die Realisationen der Rohstoffpreise.

Werden den Reservenbesitzern rationale Erwartungen hinsichtlich der Preise auf dem Rohstoffmarkt unterstellt und gelte wieder für die Nachfrage $p(t)=N(q(t)) u(t)$ mit $N^{\prime}(q(t))<0$ und $E[u(t)]=1$, dann ist

$$
\alpha(t)+\beta(t) N(q(t)) \Gamma_{R^{-1}}=\lambda e^{\delta t} E\left(U_{R}^{\prime}\right)^{-1} .
$$

Hierin ist $\Gamma_{R}$ der nach 6.71 definierte Risikobias der Reservenbesitzer. 
Ein risikoaverser Reservenbesitzer setzt nicht den Erwartungswert des Rohstoffpreıses in seinem Margınalkalkul zur Ermıttlung seınes Angebots an, sondern einen nach unten mit $\Gamma_{R}>1$ korrigierten Erwartungswert. Das Angebot an Abbaurechten ıst gerınger als beı Risıkoneutralität.

Die Nachfrage nach Abbaurechten wird wieder erhalten aus den Optimierungskalkülen der Abbauunternehmer, die nun lauten:

$$
\begin{aligned}
& \max _{(q(t))} E\left\{U_{A}\left[{ }_{0} \delta^{\top}(p(t)-c-[\alpha(t)+B(t) p(t)]) q(t) e-\delta t d t\right]\right\} \\
& \text { u.d.B. } q(t) \geq 0 .
\end{aligned}
$$

Ein positives $q(t)$ liefert die notwendige Bedingung

$$
E\left[U_{A^{\prime}} p(t)\right][1-B(t)]-E\left(U_{A^{\prime}}\right)[C+\alpha(t)]=0 .
$$

Hieraus folgt für gegebene Rohstoffnachfrage $p(t)=N(q(t)) u(t)$ bei rationalen Erwartungen der Abbauunternehmen

$$
[1-B(t)] N(q(t))=\Gamma_{A 2}[c+\alpha(t)],
$$

mit $\Gamma_{A 2}$ als Risikobias des Abbauunternehmers, der nun aufgrund der vom Rohstoffpreis abhängigen Abbaurechte unterschiedlich ist vom Risikobias $\Gamma_{A}{ }^{1)}$ bei deterministischen Preisen für Abbaurechte.

Aus 6.87 und 6.90 folgt für das Gleichgewicht auf dem Markt für Abbaurechte

1) Das $\Gamma_{A}$ des $A$ bschnittes 6.2.1.1. wird für den folgenden Vergleich mit $\Gamma_{A 1}$ bezeichnet. 


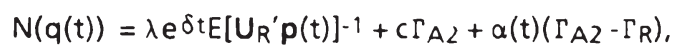

woraus dıe gleıchgewichtıge Entwicklung der Abbaumengen und Mengen der Abbaurechte erhalten wird:

$$
d q / d t=\delta \lambda e^{\delta t}\left\{N^{\prime}(q(t)) E\left[U_{R}^{\prime} p(t)\right]\right\}-1<0
$$

Mit 6.91 folgt

$$
d q / d t=\delta\left[N(q(t))-c \Gamma_{A 2}-\alpha(t)\left(\Gamma_{A 2}-\Gamma_{R}\right)\right] N^{\prime}(q(t))-1 .
$$

Die beiden Großen $\Gamma_{A 2}$ und $\Gamma_{R}$ sind abhängig von der Wahl der Parameter $\alpha(t)$ und $B(t)$. Werden $\alpha(t)$ und $B(t)$ paretoeffizient gewählt, dann gilt $\Gamma_{A 2}=\Gamma_{R}$. Dies folgt aus einem Ansatz der Art: 1)

$$
\begin{aligned}
& \left.\max E\left\{U_{A} l_{0} \int^{\top}(p(t)-c-[\alpha(t)+B(t) p(t)]) q(t) e-\delta t d t\right]\right\} \\
& (\alpha(t), B(t)) \\
& \text { u.d.B. } \\
& E\left\{U_{R}\left[{ }_{0} \int^{\top}[\alpha(t)+B(t) p(t)] q(t) e^{-\delta t d t}\right]\right\} \geq U_{R}{ }^{*}, \\
& \alpha(t), B(t) \geq 0 \text {. }
\end{aligned}
$$

Die Abbauunternehmen optimieren ihren Erwartungsnutzen unter der Restriktion, den Reservenbesitzern ein bestimmtes Mindestnutzenniveau $U_{R}$ * zukommen zu lassen. 2 )

1) Vgl. zu einem ähnlichen Ansatz Sutinen (1980), S. 173.

2) Mit diesem Ansatz dürfte die Situation zwischen den Fördergesellschaften der Industrienationen und den rohstoffbesitzenden Entwicklungsländern vor 1960 beschrieben sein. Zwischen 1960 und 1970 fand eine Umschichtung der Verfügungsrechte über Reserven zwischen den Gesellschaften und den Rohstoffländern statt. Nach 1970 könnte die Situation durch einen Ansatz beschrieben werden, in dem die Rohstoffländer (vorrangig Erdölförderländer) ihren Erwartungsnutzen maximieren unter der Restriktion eines Mindestnutzenniveaus für die Abbauunternehmen und ihres Ressourcenbestandes. 
An notwendigen Bedingungen werden erhalten ")

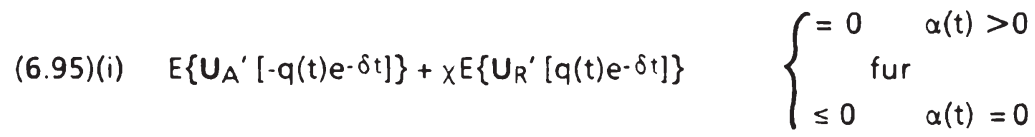

(6.95)(ii) $E\left\{U_{A^{\prime}}\left[-p(t) q(t) e^{-\delta t}\right]\right\}+X E\left\{U_{R}^{\prime}\left[p(t) q(t) e^{-\delta t}\right]\right\} \begin{cases}=0 & B(t)>0 \\ & \text { furr } \\ \leq 0 & B(t)=0 .\end{cases}$

Beide Bedingungen liefern

$$
E\left(U_{A^{\prime}}\right) E\left[U_{A^{\prime}} p(t)\right]^{-1}=E\left(U_{R}^{\prime}\right) E\left[U_{R}^{\prime} p(t)\right]^{-1} \Leftrightarrow \Gamma_{A 2}=\Gamma_{R}
$$

Gleichung 6.93 geht mit diesem Ergebnis über in 2)

$$
d q / d t=\delta\left[N(q(t))-c \Gamma_{A 2}\right] N^{\prime}(q(t))^{-1}
$$

Die Entwicklung der Abbaumengen beı der mengenbezogenen Förderabgabe erfolgte gemaß Gleıchung 675 .

$$
d q / d t=\delta\left[N(q(t))-c \Gamma_{A 1}\right] N^{\prime}(q(t))-1
$$

Wegen $\Gamma_{A 1}>\Gamma_{A 2^{3}}$ für $\alpha(t)>0$ und $0<B(t)<1$ gilt die Aussage:

Ein indexierter Reservenpreis (d.h. eine mengen- und wertbezogene Forderabgabe) fuhrt zu eınem schnelleren Abbau der Ressourcenläger als ein deterministischer Preis für Abbaurechte (d.h. eıne mengenbezogene För-

1) Die Lagrangefunktion dazu lautet

$$
\begin{aligned}
L=E\left\{U_{A}\left\{{ }_{0}{ }^{\top}(p(t)-c-[\alpha(t)+B(t) p(t)]) q(t) e^{-\delta t d t}\right\}\right\}+ \\
x\left\{E\left\{U_{R}\left\{{ }_{1}{ }^{\top}[\alpha(t)+B(t) p(t)] q(t) e^{-\delta}[d t\}\right\}-U_{R^{*}}\right\} .\right.
\end{aligned}
$$

2) Vgl. Sutinen (1980), S 174

3) Vgl. Anhang VIII. 
derabgabe). Denn mittels der Indexierung des Reservenpreises erfolgt im Gegensatz zum deterministischen Reservenpreis eine Verteilung des Risikos auf Reservenbesitzer und Abbauunternehmer. Für den Abbauunternehmer sinkt damit das Risiko und es findet als Reaktion darauf eine Umverteilung des Ressourcenabbaus zugunsten der Gegenwart statt.

Eine komparative Dynamik bezüglich der Auswirkungen einer Risikoänderung führt tendenziell zu denselben Ergebnissen wie die Analyse im Abschnitt zuvor.

Anhand der Gleichungen 6.91 bzw. 6.93 können die Auswirkungen einer paretoineffizienten Wahl der Parameter $\alpha(t)$ und $B(t)$ diskutiert werden. Paretoeffizıenz implizierte gleichen Risikobias für den Reservenbesitzer und den Abbauunternehmer. Das Ausmaß und die Richtung der Abweichung von einer in diesem Sinne paretoeffizıenten Ressourcenallokation wird bestimmt von dem Vorzeıchen und der Größe der Differenz der Risikobiases (Anhang VIII).

Bezeichne $(d q / d t)^{*}, q(t)^{*}$ die intertemporale Ressourcenallokation bei paretoeffizienter Wahl von $\alpha(t)$ und $\beta(t)$, dann folgt für die Allokation $d q / d t$, $q(t)$ bei paretoineffizient gewählten Parametern:

$$
\Gamma_{A 2}-\Gamma_{R}>0 \Rightarrow q(t)^{\star}>q(t)>0 \text { und }(d q / d t)^{\star}<d q / d t<0,
$$

$$
\Gamma_{A 2}-\Gamma_{R}<0 \Rightarrow 0<q(t)^{\star}<q(t) \text { und } 0>(d q / d t)^{\star}>d q / d t \text {. }
$$

Der größere Risikobias des Abbauunternehmers (Reservenbesitzers) führt .zu einem langsameren (schnelleren) Abbau der Ressourcenläger.

Hier einzuordnen sind auch die Fälle, in denen Handelspartner mit unterschiedlichem Risikoverhalten gegenüberstehen:

Risikoaverse Abbauunternehmer und risikoneutrale Reservenbesitzer, Fall (i);

Risikoneutrale Abbauunternehmer und risikoaverse Reservenbesitzer, Fall (ii). 


\subsubsection{Modelle zur Abbaumengenteilung}

Im Gegensatz zur Konzession werden die Reservenbesitzer für die Abgabe von Nutzungsrechten für Reservenbestande nun nıcht nomınal, sondern real in Form von Rohstoffmengen entlohnt. 1) Mit den erhaltenen Rohstoffmengen treten die Reservenbesitzer als Anbieter auf dem Rohstoffmarkt in Konkurrenz mit den Abbauunternehmern. Je nach Anzahl der Reservenbesitzer und der Abbauunternehmer kann es durch die Abbaumengenteilung zu unterschiedlichen Konkurrenzsituationen auf der Angebotsseite des Rohstoffmarktes kommen. Für die Nachfrageseite des Rohstoffmarktes gelte weiterhin eine polypolistische Struktur.

(1) Herrscht sowohl auf der Angebots- als auch auf der Nachfrageseite des Reservenmarktes eine polypolistische Struktur, dann folgt für den Rohstoffmarkt ebenfalls die Marktform des Angebotspolypols.

(2) Die Situation eines bilateralen Monopols auf dem Reservenmarkt führt durch Abbaumengenteilung zu einem Angebotsduopol auf dem Rohstoffmarkt.

(3) Steht ein Reservenbesıtzer einer Vielzahl von Abbauunternehmen gegenüber, so erhält man durch Abbaumengenteilung je nach Größe der Anteile die Marktformen des Polypols oder eines marktbeherrschenden Unternehmens (Reservenbesitzer) mit "Konkurrenzring".

(4) Die Abbaumengenteilung führt zu denselben Marktformen wie unter (3), wenn man einem Abbauunternehmen eine VielzahI von Reservenbesitzern gegenüberstellt. Der Unterschied besteht darin, daß nun das Abbauunternehmen die marktbeherrschende Stellung einnehmen kann.

1) Siehe dazu Abschnitt 4.2.2. 
Eine modelltheoretische Analyse wird in den beiden nächsten Abschnitten für die unter (1) (Polypol) und unter (2) (Duopol) genannten Marktformen durchgeführt. Zu Modellen, die in einem einstufigen Marktkonzept für den Rohstoffmarkt eine Angebotsstruktur unterstellen, wie sie in (3) bzw. (4) genannt wurden, sel auf Hnyilicza/Pındyck (1976), Pındyck (1978) und Gilbert (1978) verwiesen.

\subsubsection{Polypol auf Rohstoff-und Reservenmarkt}

Sowohl auf der Angebots- als auch auf der Nachfrageseite herrsche eine polypolistische Struktur. Für jeden Reservenbesitzer und Abbauunternehmer gelten die in 6.2.1. anfangs gemachten Annahmen mit der nun geänderten Entlohnung des Nutzungsrechtes für Reserven.

Für einen gegebenen Anteil $\xi(t) \in(0,1) 1)$ an den Fördermengen der $A b$ bauunternehmen lautet die Problemstellung der Reservenbesitzer:

$$
\begin{aligned}
& \max _{(q(t))} E\left\{U _ { R } \left[{ }_{0} S^{\top} \xi(t) q(t) p(t) e^{-\delta t d t]\}}\right.\right. \\
& \text { u.d.B. } \quad R_{0} \geq{ }_{0} \int^{\top} q(t) d t, R_{0} \text { gegeben, } q(t) \geq 0 .
\end{aligned}
$$

Es folgt für die notwendigen Bedingungen:

1) $\xi(t)$ stellt sich als ein Gleichgewichtswert auf dem Rohstoffmarkt ein, bei dem für gegebene Erwartungen über Rohstoffpreise das Angebot an und die Nachfrage nach Nutzungsrechten für Reserven gleich sind. 
$(6.99)(1) \quad E\left[U_{R}^{\prime} \xi(t) p(t)\right] e^{-\delta t-\lambda}$

$$
\begin{aligned}
& \left\{\begin{array}{lrr}
=0 & q(t)>0 \\
& \text { für } & \\
\leq 0 & q(t)=0
\end{array}\right. \\
& \left\{\begin{array}{lll}
=0 & \lambda>0 & \\
& \text { für } & \\
\geq 0 & \lambda & =0 .
\end{array}\right.
\end{aligned}
$$

Das Angebot an Abbaurechten wird damit zu

(6.100) $\left.\quad E\left[U_{R}^{\prime} p(t)\right] \xi(t)=\lambda e^{\delta t} b z W .1\right)$

(6.100') $\xi(t) N(q(t)) \Gamma_{R 3^{-1}}=\lambda e^{\delta t} E\left(U_{R}\right)^{-1}$.

Der risikoaverse Ressourcenbesitzer setzt auch hier einen um den Risikobias $\Gamma_{R 3}>1$ korrıgierten, erwarteten Erlös an. Zur Folge hat dies ein geringeres Angebot an Abbaurechten.

Bei den Abbauunternehmen ist nun zu optimieren

(6.101) $\max E\left\{U_{A}\left[{ }_{0}{ }^{\top}(p(t)-c-\xi(t) p(t)) q(t) e^{-\delta t d t}\right]\right\} \quad$ u.d.B. $q(t) \geq 0$. $(q(t))$

Im Erwartungsnutzenmaxımum gılt dann für die Nachfrage nach Abbaurechten

(6.102) $E\left\{U_{A^{\prime}}(p(t)[1-\xi(t)]-c) e^{-\delta t}\right\}=0$ für $q(t) \geq 0$, bzw.

(6.102') $N(q(t))=\xi(t) N(q(t))+C \Gamma_{A 3}$.

1) Es gelten wieder die Annahmen, rationale Erwartungen und $E[p(t)]=N(q(t))$. $\Gamma_{R 3}$ bzw. später $\Gamma_{A 3}$ stehen für die Risikobiases bei Abbaumengenteilung. 
Zusammen mit 6.100' liefert 6.102' als erwarteten Gleichgewichtspreis des Rohstoffmarktes in $\mathrm{t}$

$$
N(q(t))=\lambda e^{\delta t} E\left[U_{R}^{\prime} u(t)\right]^{-1}+c \Gamma_{A 3}
$$

und für den zeitlichen Verlauf der gleichgewichtigen Rohstoffmengen

$$
d q / d t=\delta\left[N(q(t))-c \Gamma_{A 3}\right] N^{\prime}(q(t))^{-1}<0 .
$$

Aus Anhang VIII (den Gleichungen VIII.1 und VIII.2) wird im Vergleich mit einer mengen- und wertbezogenen Förderabgabe die Relation erhalten 1)

$$
(d \alpha / d \beta)_{\alpha>0, \beta>0}>(d \alpha / d \beta)_{\alpha=0, B>0} \Leftrightarrow \Gamma_{A 2}>\Gamma_{A 3} .
$$

Für einen gegebenen Erwartungswert des Rohstoffpreises im Zeitpunkt t wird ein risikoaverses Abbauunternehmen im Falle der Abbaumengenteilung eine größere Fördermenge ausbringen als im Falle einer mengen- und wertbezogenen Förderabgabe (Gleichung 6.103).

Weiter führt eine Abbaumengenteilung zu einem Abbaupfad, der eine stärkere zeitliche Veränderung aufweist als die Pfade bei den Konzessionsmodellen $\left(\Gamma_{A 1}>\Gamma_{A 2}>\Gamma_{A 3}\right)$. 2) Abbaumengenteilung führt zu einem schnelleren Abbau der Ressourcenbestände.

$\mathrm{Zu}$ begründen ist dieses Verhalten ebenfalls mit einer Senkung des Risikos für den Abbauunternehmer. Dadurch, daß er real in Form von Abbaumengen die Nutzung der Reserven entlohnt, überträgt er an den Reservenbesitzer auch das Preisrisiko.

Eine Variation des Risikos liefert auch hier Auswirkungen auf die Fördermengen und deren zeitliche Veränderungen, die in dieselbe Richtung weisen wie die Ergebnisse des Abschnittes 6.2.1.

\footnotetext{
1) Die Abbaumengenteilung in diesem Modellzusammenhang führt zu demselben Ergebnis wie eine wertbezogene Forderabgabe.

2) Vgl.dazu S.227.
} 


\subsubsection{Duopol auf dem Rohstoffmarkt}

Zwischen einem Reservenbesitzer und einem Abbauunternehmer bestehe auf dem Reservenmarkt die Situation eines bilateralen Monopols. Die Abbaumengenteilung führe auf dem Rohstoffmarkt zu der Angebotsstruktur eines Duopols.

Sowohl der Reservenbesitzer als auch der Abbauunternehmer maximieren ein Kalkül, wie es in 6.98 bzw. 6.101 gegeben ist, nun allerdings unter Berücksichtigung des erwarteten Nachfrageverlaufs. Hinsichtlich des Lösungskonzeptes für die Duopolisten gelten die Annahmen des Abschnitts 3.3.

Die notwendigen Bedingungen für die Erwartungsnutzenmaxima der beiden Duopolisten lauten

für den Reservenbesitzer

$$
E\left\{U_{R}{ }^{\prime} \xi(t)\left[p(t)+q(t) N^{\prime}(q(t)) u(t)\right]\right\} e^{-\delta t}=\lambda \text { für } q(t) \geq 0
$$

und für den Abbauunternehmer

$$
E\left\{U_{A^{\prime}}\left\{[1-\xi(t)]\left[p(t)+q(t) N^{\prime}(q(t) u(t)]-c\right\} e^{-\delta t}\right\}=0 \text { für } q(t) \geq 0 .\right.
$$

Bei gegebener Erwartungshaltung hinsichtlich der Nachfrage nach Rohstoffen, gegebenem Risikoverhalten und für ein gegebenes $\gamma(t)$, steht 6.106 für die Reservennachfrage. Umgeschrieben erhält man für die beiden Marktseiten

$$
\xi(t) \Gamma_{R 3^{-1}}\left[N(q(t))+N^{\prime}(q(t)) q(t)\right]=\lambda e^{\delta t E\left(U_{R}^{\prime}\right)-1}
$$

und

(6.107') $[1-\xi(t)] \Gamma_{A 3^{-1}}\left[N(q(t))+N^{\prime}(q(t)) q(t)\right]=c$.

Hieraus folgt für das Gleichgewicht auf dem Reservenmarkt die Beziehung

$$
N(q(t))+N^{\prime}(q(t)) q(t)=c \Gamma_{A 3}+\Gamma_{R 3} \lambda e^{\delta t} E_{(}\left(U_{R}^{\prime}\right)-1
$$


und weiter für die zeitliche Entwicklung der gleichgewichtıgen Handelsmengen

$$
\begin{aligned}
d q / d t= & \delta\left[N(q(t))+N^{\prime}(q(t)) q(t)-c \Gamma_{A 3}\right] \\
& \left\{2 N^{\prime}(q(t))+\lambda e^{\left.\delta t E\left(U_{R}^{\prime}\right)-2\left[d E\left(U_{R}^{\prime}\right) / d q\right]\right\}-1<0}\right.
\end{aligned}
$$

bzw. der erwarteten und realisierten Rohstoffpreise

$$
d p / d t=N^{\prime}(q(t))(d q / d t)>0
$$

Verglichen mit der Allokation im Polypol (Gleichung 6.104) führt die Abbaumengenteilung bei duopolistischer Angebotsstruktur zu einem monopolähnlichen Allokationsbias. Die konservierende Wirkung des Duopols im Vergleich zum Polypol bleibt unter der Abbaumengenteilung erhalten.

Die Gleichung 6.109 kann auch als das Abbauprofil eines Monopolisten auf dem Rohstoffmarkt ohne Abbaumengenteilung betrachtet werden. Dann allerdings ist das $\Gamma_{A}$ dieses Monopolisten größer als das in 6.109 angegebene $\Gamma_{A 3} .{ }^{1)} D . h$. der Abbaupfad des Monopolisten verläuft flacher als der $A b$ baupfad einer durch Abbaumengenteilung induzierten duopolistischen Angebotsstruktur.

1) Vgl. hierzu Gleichung 6105. 


\subsubsection{Ein Modell zum Dienstleistungsvertrag}

Der Dienstleistungsvertrag (Abschnitt 4.2.2.) wird als Ansatz der neueren Wettbewerbstheorie modelliert. In Abschnitt 2.1.3. wurde ein solcher nutzenorientierter Ansatz unter Sicherheit dargestellt. Diesen Ansatz gilt es hier auf Risikosituatıonen zu erweitern.

In einem Dienstleistungvertrag übernimmt ein Abbauunternehmen die Fördertätigkeit gegen Entgelt. Als Gegenleistung stellt es dem Reservenbesitzer die Dienstleistung der Unternehmensführung, die Nutzung von Technologien und die Dienstleistung der Vermarktung der Abbaumengen zur Verfügung.

Der Reservenbesitzer vergibt hier keine Abbaurechte zur Nutzung eines Ressourcenbestandes und das Abbauunternehmen erwirbt keine Abbaurechte. Ein Reservenmarkt existiert hier nicht.

Auf dem Rohstoffmarkt tritt das Abbauunternehmen als Anbieter auf. Es stehe mit anderen Abbauunternehmen unter vollkommener Konkurrenz der Nachfrage $p(t)=N(q(t)) u(t)$, mit $N^{\prime}(q(t))<0, N^{\prime \prime}(q(t))=0$ und $E(u(t))=1$ nach Rohstoffen gegenüber.

Die Abbaukosten c pro Rohstoffeinheit seien fix. Das Abbauunternehmen wählt zu den erwarteten Preisen $E[p(t)]$ für den Rohstoffmarkt sein Angebot $q(t)$. Das Abbauunternehmen erhält pro Periode das Entgelt $A(t)$.

Zielsetzung der Manager ist es, den Erwartungsnutzen über dem Abbauzeitraum zu maximieren. Das Zielfunktional setzt sich nun aus zwei Funktionen zusammen:

Einer Periodennutzenfunktion $V[A(t)]$, wie sie in Abschnitt 2.1.3. eingefuhrt wurde mit $V_{A}{ }^{\prime}>0, V_{A}{ }^{\prime \prime}<0$ und einer "spezifischen Risikopräferenz- 
funktion" $U_{D}$ "

$$
U_{D}=U_{D}\left\{{ }_{0} f^{\top} V[A(t)] e^{-\delta t} d t\right\},
$$

wie sie in den Abschnitten des dritten Kapitels Verwendung findet. Beide Funktionen sind als zweimal stetig differenzierbare, konkave Funktionen unterstellt worden.

Aus der Abbautätigkeit resultiert ein Gewınn $G(t)=p(t) q(t)-c q(t)-A(t)$, der an den Reservenbesitzer ausgeschüttet wird. Vom Reservenbesitzer wird über den Abbauzeitraum ein Mindestgewinn $G_{0}$ gefordert. Mit $p(t)$ werden auch die Gewinnausschüttungen zu Zufallsvariablen. Die Forderung von risikoaversen Reservenbesitzern nach einem Mindestgewinn $G_{0}$ ist zu bewerten mit deren Risikopräferenzfunktion $U_{B}$ und bedeutet, daß der Erwartungsnutzen des Gegenwartswertes der gesamten Gewinnauschüttungen im Abbauzeitraum ein durch $G_{0}$ bestimmtes Nutzenniveau $U_{B}\left(G_{0}\right)$ nicht unterschreiten darf:

$$
U_{B}\left(G_{0}\right) \leq E\left\{U\left[0 \int^{\top} G(t) e^{-\delta t} d t\right]\right\} .
$$

Mit diesen Annahmen stellt sich dem unternehmensführenden Management das Optimierungsproblem:

(6.111)

$$
\begin{aligned}
& \max _{(q(t), G(t), T)} E\left\{U_{D}\left\{{ }_{0} T^{\top} V[A(t)] e^{-\delta t} d t\right\}\right\} \\
& \text { u.d.B. } \quad R_{0} \geq{ }_{0}^{\top} q(t) d t, R_{0} \text { gegeben, } q(t) \geq 0, G(t) \geq 0 \\
& U_{B}\left(G_{0}\right) \leq E\left\{U_{B}\left[\int_{0} S^{\top} G(t) e^{-\delta t} d t\right]\right\}, G_{0} \text { gegeben. }
\end{aligned}
$$

Es ergeben sich mit der Lagrangefunktion

1) Zu diesem Begriff siehe Sinn (1980), S 246. 


$$
\begin{aligned}
L= & E\left\{U_{D}\left[{ }_{0} \int^{\top} V[A(t)] e^{-\delta t} d t\right]\right\}+\lambda\left[R_{0}{ }^{-}{ }_{i j} J^{\top} q(t) d t\right]+\mu\left\{E\left\{U_{B}\left[_{0} S^{\top} G(t) e^{-\delta t} d t\right]\right\}-\right. \\
& \left.U_{B}\left(G_{0}\right)\right\}{ }^{1)}
\end{aligned}
$$

die notwendigen Bedingungen

(6.112)(i) $E\left\{U_{D}{ }^{\prime} V_{A}^{\prime}[p(t)-c]\right\} e^{-\delta t}-\lambda$

$$
\begin{aligned}
& \begin{cases}=0 & q(t)>0 \\
& \text { für } \\
\leq 0 & q(t)=0\end{cases} \\
& \begin{cases}=0 & G(t)>0 \\
\leq 0 & \text { für } G(t)=0\end{cases} \\
& \left\{\begin{array}{llll}
=0 & & \lambda>0 \\
& \text { für } & \\
\geq 0 & & \lambda & =0
\end{array}\right. \\
& \text { (6.112)(iv) } E\left\{U_{B}\left[\int_{0} S^{\top} G(t) e^{-\delta t} d t\right]\right\}-U_{B}\left(G_{0}\right) \quad\left\{\begin{array}{llll}
=0 & & \mu & >0 \\
& \text { für } & \\
\geq 0 & \mu & =0
\end{array}\right.
\end{aligned}
$$

(6.112)(v) $E\left\{U_{D}{ }^{\prime} V[A(T)]\right\} e^{-\delta T}-\lambda q(T)+\mu E\left\{U_{B}{ }^{\prime}[G(T)]\right\} e^{-\delta t}=0$

1) Zu beachten ist, daß gilt: $A(t)=[p(t)-c] q(t)-G(t)$. 
Für positive Abbaumengen $q(t)$ und Gewınnausschüttungen $G(t)$ gılt:

(6.113)(i) $E\left[U_{D} V^{\prime}{ }_{A} p(t)\right] E\left[U_{D}{ }^{\prime} V^{\prime}{ }_{A}\right]^{-1}-C=\lambda e^{\delta t} E\left[U_{D} V^{\prime}{ }_{A}\right]^{-1}$

(6.113)(ii) $E\left[U_{D}{ }^{\prime}{ }^{\prime} A\right]=\mu E\left(U_{B}{ }^{\prime}\right)$

und weiter 1)

(6.114)(i) $E[p(t)]+\operatorname{cov}\left[U_{D} V^{\prime}{ }_{A}, p(t)\right] E\left[U_{D} V^{\prime}{ }_{A}\right]^{-1}-c=\lambda e^{\delta t E\left[U_{D}{ }^{\prime} V^{\prime}{ }_{A}\right]^{-1}}$

(6.114)(ii) $E\left[U_{D}{ }^{\prime}{ }^{\prime}{ }_{A}\right] E\left(U_{B}\right)^{\prime-1}=\mu$.

Der mit der Risikopräferenz der Reservenbesitzer bewertete Schattenpreis der Gewinnausschüttungen ist gleich dem mit der Risikopräferenz des Managements bewerteten Grenznutzen der Entlohnung. In Gegenwartswerten ist der Schattenpreis der Gewinnausschüttung konstant. Für einen Zeitpunkt $t$ wählt das Management jene Abbaumenge, bei der der erwartete Marktpreis des Rohstoffes abzüglich der Kosten für Risikoverhalten und Abbau gleich ist den Opportunitätskosten der Ressourcennutzung.

Zur Diskussion des resultierenden Abbauprogrammes wird Gleichung 6.114 mittels der Variablen $\Gamma_{D}=E[p(t)]\left\{E[p(t)]+\operatorname{cov}\left[U_{D}{ }^{\prime} V^{\prime}{ }_{A}, p(t)\right] E\left[U_{D}{ }^{\prime} V^{\prime}{ }_{A}\right]^{-1}\right\}-1$ umgeschrieben zu

$$
\Gamma_{D^{-1}} E[p(t)]-c=\lambda e^{\delta t} E\left[U_{D}{ }^{\prime}{ }^{\prime}{ }_{A}\right]^{-1}
$$

$\Gamma_{D}$ steht auch hier für den durch Risiko verursachten Bias im Angebotsverhalten des Abbauunternehmens. Wenn der Dienstleistungsvertrag Risiko und/oder Risikoaversion mindert, dann ist $\Gamma_{D}$ kleiner als ein $\Gamma_{G}$, das der unternehmensführende Manager ansetzen würde, falls er als Reservenbesitzer die Ressource in eigener Regie abbauen würde. Gemäß der bisherigen Argumentationsweise ist zu erwarten, daß auch der Dienstleistungsvertrag eine Korrektur des Risikobias bewirkt.

1) Es gelten die Annahmen $V_{A^{\prime}}>0$ und $V_{A}^{\prime \prime}<0$ 
Aus 6.115 folgt für die zeitliche Entwicklung der Fördermengen $q(t)$ und der Entlohnung $A(t)$ bei rationalen Erwartungen des Managements hınsichtlich der Rohstoffpreise auf den zukünftigen Kassamärkten

$$
\begin{aligned}
{\left[\Gamma_{D^{-1}} N^{\prime}(q(t))-c\right](d q / d t) } & =\delta \lambda e^{\delta t} E\left[U_{D^{\prime}} V^{\prime}{ }_{A}\right]^{-1}- \\
\lambda e^{\delta t} E\left[U_{D}{ }^{\prime} V^{\prime}{ }_{A}\right]^{-2}\left\{d E\left[U_{D}{ }^{\prime} V^{\prime}{ }_{A}\right] / d A\right\} & (d A / d t) .
\end{aligned}
$$

Im Optimum gilt intertemporal auch Gleichung 6.114, aus der folgt

$$
d E\left[U_{D}{ }^{\prime}{ }^{\prime}{ }_{A}\right] / d t=E\left(U_{B}\right)(d \mu / d t)+\mu\left[d E\left(U_{B}{ }^{\prime}\right) / d t\right] .
$$

Da sowohl $d \mu / d t=0$ als auch $d E\left(U_{B}{ }^{\prime}\right) / d t=0$ ist, hat man $d E\left[U_{D}{ }^{\prime}{ }^{\prime} A\right] / d t=0$.

Ferner folgt wegen $d U_{D^{\prime}} / d t=0$ und $V_{A}{ }^{\prime \prime}<0$ auch $d A / d t=0$. Mit $d A / d t=0$ wird $6.116 \mathrm{zu}$

$$
d q / d t=\delta\left[N(q(t))-c \Gamma_{D}\right]\left[N^{\prime}(q(t))-c \Gamma_{D}\right]^{-1}<0 .
$$

Die Risikopräferenz der Reservenbesitzer $\left(U_{B}\right)$ hat keinen Einfluß auf das Abbauprofil. Entscheidend ist die Risikopräferenz des Managers und der Einfluß, den der Dienstleistungsvertrag auf das Risikoverhalten hat. Es kann direkt abgeleitet werden, daß der unter Dienstleistungsvertrag stehende Manager einen gegebenen Ressourcenbestand schneller abbaut als dies ein gewinnorientierter Unternehmer in eigener Regie tun würde. Denn die risikomindernde Wirkung des Dienstleistungsvertrages hat eine Umverteilung der Abbaumengen zugunsten gegenwartsnäherer Perioden zur Folge:

$$
(d q / d t)_{D}<(d q / d t)_{G}<0 \Leftrightarrow 1<\Gamma_{D}<\Gamma_{G} .
$$




\section{Zusammenfassung der Ergebnisse}

Ziel der Arbeit war es, eine Analyse der intertemporalen Allokationswirkungen von Marktsubstituten zu liefern, insbesondere langfristiger Lieferverträge für Rohstoffe und Vertragsvereinbarungen zur Reservennutzung (Kapitel 5 und 6). Zu diesem Zweck war zu ermitteln, welchen Einfluß das durch fehlende Zukunftsmärkte entstehende Risiko auf das Verhalten der Abbauunternemer hat (Kapitel 3). Vorangestellt wurde der Betrachtung die Diskussion der intertemporalen Ressourcenallokation unter Sicherheit (Kapitel 2), um einerseits in einem (Literatur-) Überblick in die Marktallokation erschöpfbarer Ressourcen einzuführen und um andererseits die später unter Risiko diskutierten Modellansätze zunächst in einfacheren Modellen unter Sicherheit darzustellen.

Vergleicht man die Aussagen zu den Allokationswirkungen verschiedener Marktformen (Polypol, Monopol, Duopol) und verschiedener Zielsetzungen der Angebotsseite in einem System vollständiger Zukunftsmärkte, so ergibt sich bei Gewinnmaximierung:

Ein gegebener Ressourcenbestand wird am schnellsten von einer polypolistischen Angebotsseite und am langsamsten von einem Monopolisten erschöpft. Dazwischen liegt der Erschöpfungszeitpunkt eines Reservenbestandes, der von einem Angebotsduopol abgebaut wird. Für die Nachfrage wird in der Analyse eine lineare, zeitlich invariante Funktion unterstellt.

Hinsichtlich alternativer Zielsetzungen für eine polypolistische Angebotsstruktur erhält man die Aussage, daß eine nutzenorientierte Angebotsseite einen gegebenen Ressourcenbestand mit dem gleichen Allokationsmuster erschöpft wie eine gewinnorientierte Angebotsseite. Eine umsatzorientierte Angebotsseite hingegen baut den gleichen Ressourcenbestand innerhalb eines kleineren Zeitraumes ab. Zu begründen ist dieses Resultat mit dem Fehlen der Abbaukosten in der Zielfunktion. Der Marktpreis wächst mit der Diskontrate. Er wächst also stärker als im Falle einer 
gewinnorientierten Angebotsseite. Erst, wenn über eine bındende Gewınnrestriktion Opportunitätskosten der Gewinnausschüttung von der Unternehmensführung zu berúcksichtigen sind, sinkt die Wachstumsrate der Marktpreise unter die Diskontrate, bleibt aber größer als bei Gewınnorientierung der Abbauunternehmer.

Gibt man die Annahme existierender Zukunftsmärkte auf, so wird die Fragestellung zwar wirklichkeitsnäher, aber auch komplexer. Fehlende Zukunftsmärkte bedeuten für die Abbauunternehmer Unsicherheit über die zukünftigen Marktpreise der Rohstoffe. In der modelltheoretischen Betrachtungsweise wird dieser unvollkommene Informationsstand nicht als Unsicherheit (Unkenntnis der Verteilungsfunktion), sondern in der Form des Risikos (Kenntnis der Parameter der Verteilungfunktion) behandelt.

Wenn Risiko über die Nachfrageseite eingeführt wird (Kapitel 3), so läßt sich durchgehend für alle Marktformen zeigen, daß Risiko, genauer risikoaverses Verhalten, zu einem Allokationsbias zugunsten der Zukunft führt. Zwischen den einzelnen Marktformen bleiben die in Kapitel 2 abgeleiteten Relationen der zeitlichen Allokationsmuster erhalten.

Ein im Zeitablauf zunehmendes Risiko führt im Vergleich mit einem konstanten Risiko, wie es für das oben angeführte Resultat unterstellt wurde, zu einer Verlagerung des Abbaus zugunsten der Gegenwart. Nimmt das Risiko mit einer hinreichend großen Rate zu, dann kann der Erschöpfungszeitpunkt für eine risikoaverse Angebotsseite vor dem Erschöpfungszeitpunkt liegen, den eine risikoneutrale Angebotsseite realisieren würde.

Ursächlich für den Allokationsbias zugunsten der Zukunft im Falle eines konstanten Risikos ist die Annahme, daß die Preise der Kassamärkte im gesamten Abbauzeitraum [0, T] mit Risiko behaftet sind. Indem der risikoaverse Abbauunternehmer im Vergleich mit dem risikoneutralen $A b$ bauunternehmer sein Abbauprofil zugunsten gegenwartsfernerer Perioden verlagert, betreibt er eine intertemporale Diversifikation des Risikos, mindert er sein Risiko in Form der Varianz seines Gewinnes über dem Abbauzeitraum. 
Damit ist eine Antwort auf die Frage gefunden, wie Risıko das Intertemporale Abbauverhalten andert. Nun kann die Betrachtungsweise erweitert werden, indem man Substitute für fehlende Zukunftsmarkte zulaßt und die Allokationswirkungen von Terminkontrakten, Optıonskontrakten und Vertragsvereınbarungen analysıert (Liefervertrage für Rohstoffe und Vertrage über die Nutzung von Reserven).

Kapitel 4 gibt in deskriptiver Weise eine Darstellung real vorkommender Substitute für die fehlende Instıtution Zukunftsmarkt. Von den angeführten Institutionen Terminkontraktmarkt, Optionsmarkt und langfristıger Liefervertrag für die Allokation von Rohstoffen einerseits und den Institutionen Auktion, Vertragsvereinbarung und diskretionäre Vergabe für die Allokation von Reserven andererseits, werden die Vereinbarungen über Verträge einer ausführlicheren Betrachtung unterzogen. So sind die langfristigen Lieferverträge für Rohstoffe und die Vertragsvereinbarungen über die Nutzung von Reserven Gegenstand der modelltheoretischen Analyse in Kapitel 6.

Risiko über die Entwicklung zukünftiger Marktpreise führt in der Betrachtungsweise eines Zweiperiodenmodells (Kapitel 5) mit risikoaversen Abbauunternehmern zu einem verstärkten Abbau der Rohstoffläger in der Gegenwart (Unterkonservierung). Der Rohstoffpreis wächst mit einer Rate größer als $1+\delta$. Als Referenz dient das Allokationsergebnis mit risikoneutralen Abbauunternehmern.

Im Gegensatz zu den Mehrperiodenmodellen (open-loop Optimierung) ist der Preis des Kassamarktes in der ersten Periode des Zweiperiodenmodells keine Zufallsvariable (closed-loop Optimierung); er ist bekannt. Diese Eigenschaft des Preises ist die Ursache für den Unterschied der Allokationswirkungen von Risiko in den hier diskutierten Zwei- und Mehrperiodenmodellen.

Die Institutionalisierung von langfristigen Liefervertrajgen bzw. von Terminkontrakten führt zu einer Korrektur des genannten Allokationsbias nun zugunsten der Zukunft. Verkauft der Abbauunternehmer seinen gesamten Ressourcenbestand für $\mathrm{t}=1$ über Verträge im voraus bzw. sichert diesen 
über Hedging durch Termınkontrakttransaktionen ab (Terminkontraktund Kassapreis in $t=1$ seien gleich), dann wächst der Rohstoffpreıs mit der Rate $1+\delta$ und es stellt sich eın Allokationsergebnıs wie unter Sicherheit ein. Wird der Ressourcenbestand für $t=1$ nur teilweise uber Verträge verkauft oder uber Hedgıng abgesichert oder ist die erwartete Basıs ungleich null (Differenz zwischen dem fur $t=1$ erwarteten Terminkontraktpreis und dem für $t=1$ erwarteten Kassapreis), so stellt sich ein Allokationsbias zugunsten der Gegenwart ein.

Optionsmarkte als institutionalisierte Substitute für fehlende Zukunftsmärkte haben keine Korrekturwirkung hinsichtlich des durch Risiko entstandenen Allokationsbias. Im Gegensatz zu den zuvor genannten Marktsubstituten bewirkt die Existenz von Optionskontrakten einen stärkeren Allokationsbias zugunsten der Gegenwart, wenn die Option nicht genutzt wird. Der Rohstoffpreis wächst mit einer Rate, die nicht nur größer als $1+\delta$ ist, sondern auch größer ist als im Falle fehlender Marktsubstitute. Der "striking price" , interpretiert als Mindestpreis, bewirkt eine Übernutzung der Ressource in der Gegenwart. Wird die Option wahrgenommen, dann nimmt die Übernutzung der Ressource ab, bleibt aber immer noch stärker als in einer Situation ohne Optionskontrakte.

Positive Allokationseffekte im Sinne einer Korrekturwirkung des durch Risiko entstandenen Allokationsbıas können im Zweiperiodenmodell also nur den "langfristigen" Verträgen und mit Einschränkungen den Terminkontrakten zugeordnet werden. Optionskontrakte verstärken den Allokationsbias.

Die Analyse in Kapitel 6 (Mehrperiodenmodelle) zeigt, daß die Existenz von langfristigen Lieferverträgen in einfachster Form zu einer Menge von Folgerungen für das Angebotsverhalten eines Ressourcenbesitzers führt. Ermittelt werden die Allokationswirkungen unterschiedlicher Ausgestaltungen langfristiger Verträge. Unter bestimmten Annahmen über die Nachfrageseite kommt man auch zu Aussagen uber Marktpreise.

Werden langfristige Lieferverträge mit konstanten Periodenliefermengen und konstanten Lieferpreisen abgeschlossen, so führt dies zu 
(1) höheren Abbaumengen im Vertragszeitraum,

(2) höheren Preısen auf den Kassamärkten wàhrend der gesamten Abbautätigkeit und

(3) einer früheren Erschöpfung der Ressourcenbestände.

Steigt das Risıko auf den Kassamärkten, dann führt dies zu

(4) gerıngeren Abbaumengen über den gesamten Vertragszeitraum,

(5) einer späteren Erschöpfung der Ressourcenläger,

(6) geringeren Handelsmengen auf den Kassamärkten,

(7) höheren Vertragsmengen und

(8) höheren Preisen auf den Kassamärkten im Vertragszeitraum.

Langfristige Lieferverträge wirken damit korrigierend auf einen durch Risiko verursachten Allokatıonsbias. Der Abbauunternehmer würde ohne Lieferverträge bei fehlenden Zukunftsmärkten die Naturressource zugunsten zukünftiger Perioden verteilen (Überkonservierung). Mit dem Abschluß von Lieferverträgen nimmt er eine Umverteilung zugunsten der gegenwartsnäheren Perioden vor.

Werden variable Liefermengen und Lieferpreise im Vertragszeitraum zugelassen, dann erhält man ein Allokationsmuster, das einen gegebenen Ressourcenbestand schneller erschöpft als es bei fixen Liefermengen und Lieferpreisen der Fall wäre. Die variable Gestaltung des Liefervertrages führt zu Lieferpreisen mit positiven Wachstumsraten. Aufgrund dieser Preisentwicklung kommt es im Vergleich mit fixen Preisen zu einer Umverteilung der Abbaumengen gegenwartsferner Perioden zugunsten des Vertragszeitraumes und damit zu einer früheren Erschöpfung des Bestandes.

Neben den Vertagsvereinbarungen über Rohstoffe existieren Vertragsvereinbarungen über die Nutzung von Reserven, von denen ebenfalls Allokationswirkungen ausgehen. In einem zweistufigen Marktkonzept, das 
zWIschen Rohstoff- und Reservenmarkt unterscheidet, werden die Allokationswirkungen eıner Konzession, eines Vertrages über Abbaumengenteilung und eines Dienstleistungsvertrages analysiert. Risiko existiert nur auf dem Rohstoffmarkt. Durch vertragliche Ausgestaltung kann das Risiko auf die Teılnehmer des Reservenmarktes, Reservenbesitzer und Abbauunternehmer, verteilt werden.

Eine Konzession mit determinıstischem Reservenpreis hat im Gegensatz zu einem an den Rohstoffpreis indexierten Reservenpreis eine konservierende Wirkung. Im Falle eines polypolistischen Reserven- und Rohstoffmarktes führt die Abbaumengenteilung zu einem schnelleren Abbau eines Reservenbestandes als eine der beiden Konzessionsarten. Zu begründen ist dies mit der Risikoteilung, die im Falle der Abbaumengenteilung eins zu eins ist. Abbaumengenteilung und Duopol auf dem Rohstoffmarkt führt zu einer späteren Erschöpfung als im Falle des Polypols. Diese Relation war auch ohne Vertragsvereinbarung und auch unter Sicherheit abgeleitet worden.

Unter der Annahme, daß ein Dienstleistungsvertrag bei der unter Vertrag stehenden Unternehmung die Wahrnehmung des Risikos senkt, führt diese Vertragsvereinbarung im Vergleich mit einer selbst abbauenden, gewinnmaximierenden Unternehmung zu einer schnelleren Erschöpfung des Ressourcenbestandes. Der Dienstleistungsvertrag kann somit ebenfalls einen durch Risiko entstandenen Allokationsbias korrigieren.

Eine Variation des Risikos hat in den drei Modellansätzen zu den Vertragsvereinbarungen für Reserven tendenziell dieselbe Wirkung. Bei einer Erhöhung des Risikos sinken die Fördermengen, sinkt der Erlös der Reservenbesitzer für ein Abbaurecht und sinken betragsmäßig die Abbauraten. Steigendes Risiko wirkt konservierend auf das Abbauverhalten risikoaverser Förderunternehmer.

Die Ergebnisse der verschiedenen Modellvarianten führen zu der Aussage, daß sowohl die Substitute für fehlende Zukunftsmärkte beim Rohstoffhandel (Terminkontrakte, langfristige Lieferverträge) als auch die Substitute für den Handel von Nutzungsrechten für Reserven (Konzession mit indexiertem Reservenpreis, Abbaumengenteilung, Dienstleistungsvertrag) eine 
Korrektur des durch Risikoaversıon verursachten Allokationsbıas in Richtung auf eine Allokation bewirken, wie sie bei Risıkoneutralität der bete-ligten Wirtschaftssubjekte entstehen wurde und sozial optımal in Termını der Erwartungswerte wäre. 


\section{Anhang 1}

(zu S. 57)

Komparatıve Analyse zum Ressourcenabbau unter vollständiger Konkurrenz:

Ausgehend von den Bestimmungsgleichungen für $T$ und $r(0)$ (Gleichungen 2.61 und 2.62)

$$
(1-\delta T)[p(T)-c]=r(0)-\delta b R_{0}
$$

$$
p(T)-c=r(0) e^{\delta T}
$$

erhält man nach der Differentiation

$$
\begin{aligned}
{[p(T)-c] \delta d T+\operatorname{dr}(0)=} & -[p(T)-c] T d \delta+(1-\delta T)[d p(T)-d c]+\delta b d R_{0}+ \\
& b R_{0} d \delta+R_{0} \delta d b
\end{aligned}
$$

$$
\delta r(0) e^{\delta T} d T+e^{\delta T} d r(0)=-r(0) T e^{\delta T} d \delta+d p(T)-d c
$$

in Matrizenschreibweise

$$
\begin{aligned}
& {\left[\begin{array}{ll}
{[p(T)-c] \delta} & 1 \\
\delta r(0) e^{\delta T} & e^{\delta T}
\end{array}\right]\left[\begin{array}{c}
d T \\
d r(0)
\end{array}\right]=} \\
& {\left[\begin{array}{l}
\left\{b R_{0}-[p(T)-c] T\right\} d \delta+(1-\delta T)[d p(T)-d c]+\delta b d R_{0}+R_{0} \delta d b \\
-r(0) T e \delta T d \delta+d p(T)-d c
\end{array}\right]}
\end{aligned}
$$

mit $\Delta=\delta e^{\delta T}[p(T)-c-r(0)]>0$ 


$$
\begin{aligned}
& \text { und den Lösungen }
\end{aligned}
$$

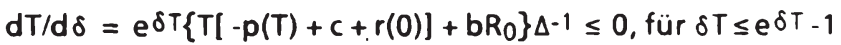

$$
\begin{aligned}
& d T / d R_{0}=e^{\delta T \delta b \Delta \Delta^{-1}>0} \\
& d T / d c=\left[e^{\delta T}(\delta T-1)+1\right] \Delta-1>0, \text { für } \delta T>1 \\
& d T / d p(T)=-\left[e^{\delta} T(\delta T-1)+1\right] \Delta^{-1}<0 \text {, für } \delta T>1 \\
& d T / d b=e^{\delta T \delta R_{0} \Delta^{-1}}>0 \\
& \operatorname{dr}(0) / d \delta=-\delta e^{\delta} \operatorname{Tr}(0) b R_{0} \Delta^{-1}<0 \\
& \operatorname{dr}(0) / d R_{0}=-\delta^{2} e^{\delta} \operatorname{Tr}(0) b \Delta^{-1}<0 \\
& \operatorname{dr}(0) / d c=-\delta^{2} e^{\delta} \operatorname{Tr}(0) T \Delta^{-1}<0 \\
& \operatorname{dr}(0) / d p(T)=\delta 2 e^{\delta} \operatorname{Tr}(0) T \Delta^{-1}>0 \\
& \operatorname{dr}(0) / d b=-\delta 2 e^{\delta} \operatorname{Tr}(0) R_{0} \Delta^{-1}<0 .
\end{aligned}
$$

\section{Anhang II}

(zu S. 61)

Der Ansatz eines rohstoffpreisabhängigen Preises für das Abbaurecht führt über die Optimierungsprobleme der Reservenbesitzer

$$
\begin{array}{ll}
\max & { }_{0} \int^{\top}[\alpha(t)+\beta(t) p(t)] q(t) e-\delta t d t \\
(q(t)) & R_{0} \geq{ }_{0} S^{\top} q(t) d t, R_{0} \text { gegeben, } q(t) \geq 0
\end{array}
$$

und der Abbauunternehmer

(II.2) $\max { }_{0} \int^{\top}\{[1-\beta(t)] p(t)-\alpha(t)-c\} q(t) e^{-\delta t} d t \quad$ u.d.B. $q(t) \geq 0$ $(q(t))$ 
zu den Angebots- und Nachfragefunktionen des Reservenmarktes

$$
\begin{array}{ll}
\alpha(t)+\beta(t) p(t)=\lambda e^{\delta t} & \text { für } q(t)>0 \\
{[1-\beta(t)] p(t)=\alpha(t)+c} & \text { für } q(t)>0,
\end{array}
$$

die als Gleichgewichtswert eine Entwicklung des Rohstoffpreises liefern gemäß

$$
p(t)=\lambda e^{\delta t}+c
$$

\section{Anhang III}

(zuS.73)

Betrachtung der Variablen $\Gamma$, die Risiko bzw. Risikoverhalten modelliert.

Es ist $E[p(t)]=N(q(t))$ und $E\left[U^{\prime} p(t)\right]=N(q(t)) E\left[U^{\prime} u(t)\right]$;

mit $\operatorname{cov}\left[U^{\prime}, p(t)\right]=E\left[U^{\prime} p(t)\right]-E\left(U^{\prime}\right) E[p(t)]$ wird $\Gamma z U$

$$
\begin{aligned}
& \Gamma=N(q(t))\left\{N(q(t))+N(q(t))\left\{E\left[U^{\prime} u(t)\right] E\left(U^{\prime}\right)^{-1}-1\right\}\right\}-1 b z w \cdot z u \\
& \Gamma=E\left(U^{\prime}\right) E\left[U^{\prime} u(t)\right]^{-1} .
\end{aligned}
$$

Daraus folgt

$$
(d \Gamma / d t) \Gamma^{-1}=\left[d E\left(U^{\prime}\right) / d t\right] E\left(U^{\prime}\right)^{-1}-\left\{d E\left[U^{\prime} u(t)\right] / d t\right\} E\left[U^{\prime} u(t)\right]^{-1} .
$$

Weiter ist

$$
\begin{aligned}
& \left\{d E\left[U^{\prime} u(t)\right] / d t\right\} E\left[U^{\prime} u(t)\right]^{-1}= \\
& \Gamma\left[d E\left(U^{\prime}\right) / d t\right] E\left(U^{\prime}\right)^{-1}+(1-\Gamma)\left\{d \operatorname{cov}\left[U^{\prime}, u(t)\right] / d t\right\} \operatorname{cov}\left[U^{\prime}, u(t)\right]^{-1},
\end{aligned}
$$

so daß folgt 


$$
\begin{aligned}
& (d \Gamma / d t) \Gamma^{-1}= \\
& (1-\Gamma)\left\{\left[d E\left(U^{\prime}\right) / d t\right] E\left(U^{\prime}\right)^{-1}-\left\{d \operatorname{cov}\left[U^{\prime}, u(t)\right] / d t\right\} \operatorname{cov}\left[U^{\prime}, u(t)\right]^{-1}\right\} .
\end{aligned}
$$

Da $\left.\operatorname{dE}\left(U^{\prime}\right) / d t\right] E\left(U^{\prime}\right)-1=0$, gilt

$$
\left.(d \Gamma / d t) \Gamma^{-1}=-(1-\Gamma)\left\{d \operatorname{cov}\left[U^{\prime}, u(t)\right] / d t\right\} \operatorname{cov}\left[U^{\prime}, u(t)\right]^{-1}\right\} .
$$

Für eınen risikoaversen Unternehmer gilt $\Gamma>1$. Variierendes Risiko bzw. sich änderndes Risikoverhalten ist in diesem Modellkontext zu unterstellen als:

$\left.(d \Gamma / d t) \Gamma^{-1}\left\{\begin{array}{c}>0 \\ =0 \\ <0\end{array}\right\} \Leftrightarrow d \operatorname{cov}\left[U^{\prime}, u(t)\right] / d t\right\} \operatorname{cov}\left[U^{\prime}, u(t)\right]^{-1}\left\{\begin{array}{l}>0 \text { zunehmendes - } \\ =0 \text { konstantes - } \\ <0 \text { abnehmendes }\end{array}\right.$

Risiko.

Diese Modellierung eines variierenden Risikos im Erwartungsnutzenansatz entspricht der von Rothschild/Stiglitz (1970) eingeführten Definition für zunehmendes Risiko: Zunehmendes Risiko liegt für ein risikoaverses Wirtschaftssubjekt dann vor, wenn für die risikoverursachende Zufallsvariable $Z$ gilt, $\operatorname{dE}(Z)=0$ bei $\operatorname{dcov}\left(U^{\prime}, Z\right)<0$.

\section{Anhang IV}

(zu S.136)

Komparative Analyse zur intertemporalen Ressourcenallokatıon unter Risiko im Zweiperiodenmodell.

Die zu differenzierenden notwendigen Bedingungen lauten

(IV.1)(i) $\quad E\left(U^{\prime}\right)(1+\delta) p_{0}=\lambda$

(IV.1)(ii) $\quad E\left(U^{\prime}\right)\left\{p_{1} e+\operatorname{cov}\left(U^{\prime}, p_{1}\right) E\left(U^{\prime}\right)^{-1}\right\}=\lambda$ 
(IV.1)(iii) $R_{0}-q_{0}-q_{1}=0$

Zur einfacheren Berechnung wird $E\left(U^{\prime}\right)$ in differenzierter Form als vierte Gleichung mit aufgenommen:

(IV.1)(iv) $d E\left(U^{\prime}\right)=$

$$
E\left\{U^{\prime \prime}\left[p_{0} q_{0} d \delta+p_{0}(1+\delta) d q_{0}+q_{0}(1+\delta) d p_{0}+q_{1} d p_{1}+p_{1} d q_{1}\right]\right\}
$$

In differenzierter Form ergeben IV.1 (i) - (iii):

$(I V .2)(i) \quad(1+\delta) p_{0} d E\left(U^{\prime}\right)+E\left(U^{\prime}\right)\left[(1+\delta) d p_{0}+p_{0} d \delta\right]=d \lambda$

(IV.2)(ii) $\quad p_{1} e d E\left(U^{\prime}\right)+E\left(U^{\prime}\right) d p_{1} e+d \operatorname{cov}\left(U^{\prime}, p_{1}\right)=d \lambda$

(IV.2)(iii) $d R_{0}-d q_{0}-d q_{1}=0$

Unter Beachtung der Nachfrageseite mit $d p_{0}=N_{0}{ }^{\prime} d q_{0}$ und $d p_{1}=N_{1}^{\prime} u_{1} d q_{1}$ wird in Matrizenschreibweise erhalten:

(IV.3)

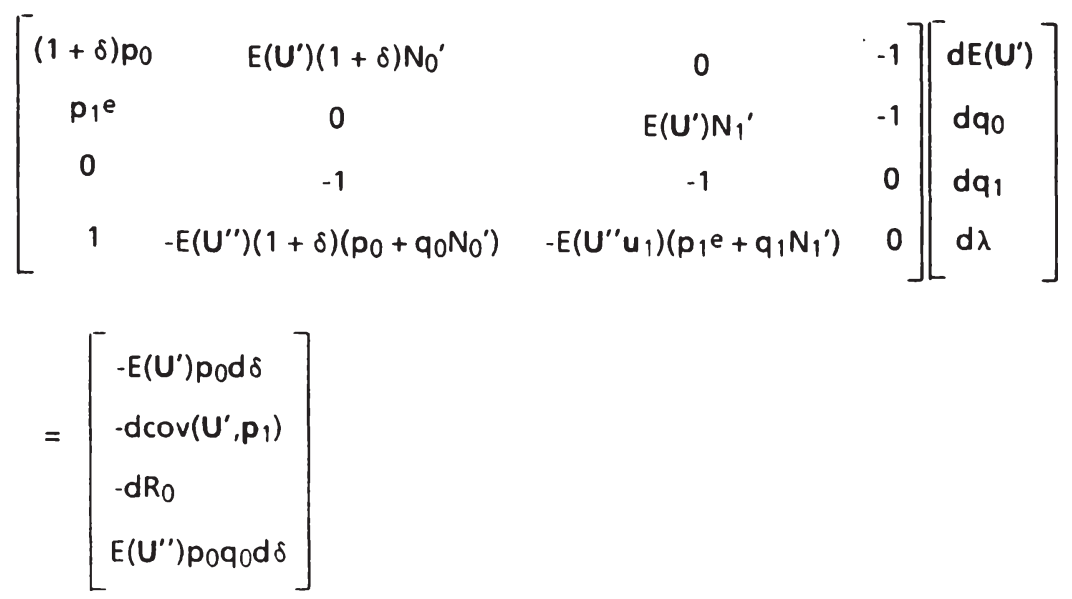


Mit

$$
\begin{aligned}
& \Delta=\left[p_{1} e-(1+\delta) p_{0}\right]\left[E\left(U^{\prime \prime} u_{1}\right)\left(p_{1} e+q_{1} N_{1}{ }^{\prime}\right)-\right. \\
& \left.E\left(U^{\prime \prime}\right)\left(p_{0}+q_{0} N_{0}\right)(1+\delta)\right]+E\left(U^{\prime}\right)\left[N_{1}^{\prime}+(1+\delta) N_{0}^{\prime}\right] ; \\
& \Delta<0 \text { für } E\left(U^{\prime \prime} u_{1}\right)\left(p_{1} e+q_{1} N_{1}{ }^{\prime}\right)<E\left(U^{\prime \prime}\right)\left(p_{0}+q_{0} N_{0}{ }^{\prime}\right)(1+\delta) \Leftrightarrow \\
& E\left(U^{\prime \prime} u_{1}\right) E\left(U^{\prime \prime}\right)-1\left(1+n_{1} e^{-1}\right) p_{1} e>\left(1+n 0^{-i}\right)(1+\delta) p_{0}
\end{aligned}
$$

wobei $E\left(U^{\prime \prime} u_{1}\right) E\left(U^{\prime \prime}\right)-1=\left\{p_{1} e+\operatorname{cov}\left(U^{\prime \prime}, u_{1}\right) E\left(U^{\prime \prime}\right)-1\right\} p_{1} e-1<1$.

Die Lösungen lauten mit $\Delta<0$ :

$$
\begin{aligned}
& d q_{0} / d \delta=-\Delta^{-1}\left\{E\left(U^{\prime}\right) p_{0}-E\left(U^{\prime \prime}\right) p_{0} q_{0}\left[p_{1} e-(1+\delta) p_{0}\right]\right\}>0 \\
& d q_{1} / d \delta=-d q_{0} / d \delta<0 \\
& d \lambda / d \delta=\Delta^{-1}\left\{E\left(U^{\prime}\right) E\left(U^{\prime \prime}\right) p_{0} q_{0}(1+\delta)\left(p_{1} e N_{0}{ }^{\prime}+p_{0} N_{1}{ }^{\prime}\right)+E\left(U^{\prime}\right) 2 N_{1} p_{0}+\right. \\
& \left.p_{1} e E\left(U^{\prime}\right) p_{0}\left[E\left(U^{\prime \prime} u_{1}\right)\left(p_{1} e+q_{1} N_{1}{ }^{\prime}\right)-E\left(U^{\prime \prime}\right)\left(p_{0}+q_{0} N_{0}{ }^{\prime}\right)(1+\delta)\right]\right\}>{ }_{<} 0 \\
& \operatorname{dq} 0 / \operatorname{dov}\left(\mathbf{U}^{\prime}, \mathbf{p}_{1}\right)=\Delta^{-1}<0 \\
& d q_{1} / d \operatorname{cov}\left(U^{\prime}, p_{1}\right)=-\Delta^{-1}>0 \\
& d \lambda / d \operatorname{cov}\left(U^{\prime}, p_{1}\right)=\Delta^{-1}\left\{( 1 + \delta ) p _ { 0 } \left[E\left(U^{\prime \prime} u_{1}\right)\left(p_{1} e+q_{1} N_{1}{ }^{\prime}\right)-\right.\right. \\
& \left.\left.E\left(U^{\prime \prime}\right)\left(p_{0}+q_{0} N_{0}^{\prime}\right)(1+\delta)\right]-E\left(U^{\prime}\right)(1+\delta) N_{0}^{\prime}\right\}><0 \\
& d q_{0} / d R_{0}=\Delta^{-1}\left\{\left[p_{1} e-(1+\delta) p_{0}\right] E\left(U^{\prime \prime} u_{1}\right)\left(p_{1} e+q_{1} N_{1}{ }^{\prime}\right)+E\left(U^{\prime}\right) N_{1}^{\prime}\right\}><0 \\
& d q_{1} / d R_{0}=-\Delta^{-1}\left\{\left[p_{1} e-(1+\delta) p_{0}\right] E\left(U^{\prime \prime}\right)(1+\delta)\left(p_{0}+q_{0} N_{0}\right)-E\left(U^{\prime}\right)(1+\delta) N_{0}^{\prime}\right\}>{ }_{<} 0 \\
& d \lambda / d R_{0}=\Delta^{-1} E\left(U^{\prime}\right)(1+\delta)\left\{N_{0}{ }^{\prime}\left[E\left(U^{\prime}\right) N_{1}^{\prime}+p_{1} e E\left(U^{\prime \prime} u_{1}\right)\left(p_{1} e+q_{1} N_{1}{ }^{\prime}\right)\right]+\right. \\
& \left.p_{0} N_{1}{ }^{\prime} E\left(U^{\prime \prime}\right)(1+\delta)\left(p_{0}+q_{0} N_{0}{ }^{\prime}\right)\right\}><0 \text {. }
\end{aligned}
$$




\section{Anhang $V$}

(zu S. 187)

Komparative Dynamik zu den Auswirkungen einer Anderung des Risikos auf das Angebots- und Abbauverhalten eınes Ressourcenbesitzers.

Die notwendigen Bedingungen lauten für $q_{v}, q_{k}(t), \lambda>0 ;$ mit $G_{q_{1}}=\partial G / \partial q_{i}$, bzw. $G_{q_{1} q_{1}}=\partial^{2} G / \partial q_{1}{ }^{2}, i=v, k$

(V.1)(i) $\quad E\left(U^{\prime}\right) G_{q_{v}}=T_{1} \lambda$

$(V .1)$ (ii) $\quad E\left(U^{\prime}\right)\left\{E\left[p_{k}(t)\right]+\operatorname{cov}\left[U^{\prime}, p_{k}(t)\right] E\left(U^{\prime}\right)^{-1}-C^{\prime}(q(t))\right\} e^{-\delta t}=\lambda$

(V.1)(iii) $R_{0}-q_{v} T_{1}-{ }_{0} \int_{2} q_{k}(t) d t=0$.

Differenziert man nach dcov $\equiv d \operatorname{cov}\left[\mathbf{U}^{\prime}, \mathbf{P}_{k}(t)\right]$, so wird erhalten:

(V.2)(i) (dE( $\left.\left.U^{\prime}\right) / d \operatorname{cov}\right) G_{q_{v}}+E\left(U^{\prime}\right)\left[G_{q_{v} q_{v}}\left(d q_{v} / d \operatorname{cov}\right)+G_{q_{v} q_{k}}\left(d q_{k} / d \operatorname{cov}\right)\right]=$ $T_{1}(d \lambda / d \operatorname{cov})$

(V.2)(ii) $\left(d E\left(U^{\prime}\right) / d \operatorname{cov}\right)\left\{E\left[p_{k}(t)\right]+\operatorname{cov}\left[U^{\prime}, p_{k}(t)\right] E\left(U^{\prime}\right)-1-C^{\prime}(q(t))\right\}+$ $E\left(U^{\prime}\right)\left\{\left\{E\left(U^{\prime}\right)-\operatorname{cov}\left[U^{\prime}, p_{k}(t)\right]\left(d E\left(U^{\prime}\right) / d \operatorname{cov}\right)\right\} E\left(U^{\prime}\right)-2-\right.$

$\left.C^{\prime \prime}(q(t))\left[\left(d q_{v} / d \operatorname{cov}\right)+\left(d q_{k} / d \operatorname{cov}\right)\right]\right\}=e^{\delta t}(d \lambda / d \operatorname{cov})$

(V.2)(iii) $-T_{1}\left(d q_{v} / d \operatorname{cov}\right)-d q_{k} / d \operatorname{cov}=0$

Zur einfacheren Darstellung wird auch hier $d E\left(U^{\prime}\right) / d$ cov nicht explizit in (i) und (ii) eingesetzt, sondern als vierte Gleichung in das System aufgenommen:

(V.2)(iv) $d E\left(U^{\prime}\right) / d \operatorname{cov}-E\left(U^{\prime \prime}\right)\left[p_{v}-C^{\prime}(q(t))\right]\left(d q_{v} / d \operatorname{cov}\right)-$ $E\left\{U^{\prime \prime}\left[p_{k}(t)-C^{\prime}(q(t))\right]\right\}\left(d q_{k} / d c o v\right)=0$.

Es ergıbt sich 
(V.3)

$$
\begin{aligned}
& {\left[\begin{array}{cccc}
E\left(U^{\prime}\right) G_{q_{v} q_{v}} & E\left(U^{\prime}\right) G_{q_{v} q_{k}} & -T_{1} & G_{q_{v}} \\
-E\left(U^{\prime}\right) C^{\prime \prime}(q(t)) & -E\left(U^{\prime}\right) C^{\prime \prime}(q(t)) & -e \delta t & E\left[p_{k}(t)\right]-C^{\prime}(q(t)) \\
-T_{1} & -1 & 0 & 0 \\
-E\left(U^{\prime \prime}\right) G_{q_{v}} & -E\left\{U^{\prime \prime}\left[p_{k}(t)-C^{\prime}(q(t))\right]\right\} & 0 & 1
\end{array}\right]\left[\begin{array}{l}
d q_{v} \\
d q_{k}(t) \\
d \lambda \\
d E\left(U^{\prime}\right)
\end{array}\right]} \\
& =\left[\begin{array}{c}
0 \\
-1 \\
-0 \\
0
\end{array}\right][d c o v]
\end{aligned}
$$

Hierin ist

$$
\begin{aligned}
G_{q_{v}} & ={ }_{0} \int^{T_{1}}\left[p_{v}-C^{\prime}(q(t))\right] e^{-\delta t d t}>0 \\
G_{q_{v} q_{v}} & =-{ }_{0} S^{T}{ }_{1} C^{\prime \prime}(q(t)) e^{-\delta t d t}<0 \\
G_{q_{v} q_{k}} & =-C^{\prime \prime}(q(t)) e^{-\delta t}<0 .
\end{aligned}
$$

Für die verschiedenen Determinanten zur Lösung nach der Cramerschen Regel wird erhalten:

$$
\begin{aligned}
& \Delta=\left(T_{1}-2\right) T_{1} E\left(U^{\prime}\right) C^{\prime \prime}(q(t))-e \delta t E\left(U^{\prime}\right) G_{q_{v}} q_{v}+\left\{T_{1}\left\{E\left[p_{k}(t)\right]-C^{\prime}(q(t))\right\}-\right. \\
& \left.e^{\delta} t G_{q_{v}}\right\}\left\{E\left(U^{\prime \prime}\right) G_{q_{v}}-T_{1} E\left\{U^{\prime \prime}\left[p_{k}(t)-C^{\prime}(q(t))\right]\right\}\right\} .
\end{aligned}
$$

Es folgt $\Delta>0$ für $T_{1} \geq 2$ und wegen Gleichung 6.24 und $\operatorname{cov}\left[U^{\prime \prime}, p_{k}(t)\right]>\operatorname{cov}\left[U^{\prime}, p_{k}(t)\right]$. 
( $V .5)$

$$
\Delta_{1}=-T_{1}<0
$$

$$
\Delta_{2}=T_{1} 2>0
$$

$$
\begin{aligned}
& \Delta_{3}=E\left(U^{\prime}\right)\left[T_{1} G_{q_{v} q_{k}}-G_{q_{v} q_{v}}\right]+G_{q_{v}}\left\{T_{1} E\left\{U^{\prime \prime}\left[p_{k}(t)-C^{\prime}(q(t))\right]\right\}-\right. \\
& \left.E\left(U^{\prime \prime}\right) G_{q_{v}}\right\} .
\end{aligned}
$$

Der letzte Summand ist negativ wegen 6.24 , denn es folgt

$$
\begin{aligned}
& E\left[p_{k}(t)\right]-C^{\prime}(q(t))>T_{1}{ }^{-1}{ }_{0} \int^{T}{ }_{1}\left[p_{v}-C^{\prime}(q(t))\right] e^{-\delta t} d t \text { und weiter } \\
& E\left(U^{\prime \prime}\right)\left\{E\left[p_{k}(t)\right]-C^{\prime}(q(t))\right\}<E\left(U^{\prime \prime}\right) T_{1^{-1}} \int_{0} \int^{T}{ }_{1}\left[p_{v}-C^{\prime}(q(t))\right] e^{-\delta t d t} .
\end{aligned}
$$

Nun gilt $E\left\{U^{\prime \prime}\left[p_{k}(t)-C^{\prime}(q(t))\right]\right\}=E\left(U^{\prime \prime}\right)\left\{E\left[p_{k}(t)\right]-C^{\prime}(q(t))\right\}+\operatorname{cov}\left[U^{\prime \prime}, p_{k}(t)\right]$.

Da cov $\left[U^{\prime \prime}, p_{k}(t)\right]<0$, gilt $E\left\{U^{\prime \prime}\left[p_{k}(t)-C^{\prime}(q(t))\right]\right\}<E\left(U^{\prime \prime}\right)\left\{E\left[p_{k}(t)\right]-C^{\prime}(q(t))\right\}$, sodaß folgt

$$
E\left\{U^{\prime \prime}\left[p_{k}(t)-C^{\prime}(q(t))\right]\right\}<E\left(U^{\prime \prime}\right) T_{1}{ }^{-1}{ }_{0} T^{T} 1\left[p_{v}-C^{\prime}(q(t))\right] e^{-\delta t d t} .
$$

Der erste Summand kann positiv oder negativ sein und bestimmt das Vorzeichen von $\Delta_{3}: \quad \Delta_{3}<0 \Leftrightarrow T_{1} G_{q_{v} q_{k}}<G_{q_{v} q_{v}}$

$$
\Leftrightarrow C^{\prime \prime}(q(t)) e^{-\delta t T_{1}}>{ }_{0} \int^{T_{1}} C^{\prime \prime}(q(t)) e^{-\delta t d t} .
$$

Bei steigendem Grenzkostenverlauf und bei abnehmenden Abbaumengen in der Zeit, wird diese Ungleichung erfüllt sein.

$$
\Delta_{4}=T_{1}\left\{T_{1} E\left\{U^{\prime \prime}\left[p_{k}(t)-C^{\prime}(q(t))\right]\right\}-E\left(U^{\prime \prime}\right) G_{q_{v}}\right\}<0 .
$$

Hieraus wird erhalten

$$
\begin{aligned}
& d q_{v} / d \operatorname{cov}=\Delta_{1} \Delta^{-1}<0, \quad d q_{k} / d \operatorname{cov}=\Delta_{2} \Delta^{-1}>0, \\
& d \lambda / d \operatorname{cov}=\Delta_{3} \Delta^{-1}<0, d E\left(U^{\prime}\right) / d \operatorname{cov}=\Delta_{4} \Delta^{-1}<0 .
\end{aligned}
$$

$D a q(t)=q_{v}+q_{k}(t)$ in $\left[0, T_{1}\right]$ ist, folgt

$$
d q / d \operatorname{cov}=d q_{v} / d \operatorname{cov}+d q_{k} / d \operatorname{cov}=T_{1}\left(T_{1}-1\right) \Delta^{-1}>0 .
$$


Ein erhöhtes Risiko oder eine erhöhte Risikoaversion führt zu einer größeren Periodenliefermenge, zu einem geringeren Kassaangebot, zu einer geringeren Abbaumenge, zu einem größeren Gegenwartswert des Schattenpreises und zu einem geringeren Erwartungsnutzen.

\section{Anhang VI}

(zu S.194)

Der komparativen Dynamik liegen zugrunde die notwendigen Bedingungen 6.37 (i) bis (iv) und die Bestimmungsgleichung für den erwarteten Grenznutzen. Die Differentiation führt zu dem System:

(VI.1)(i) (dE(U')/dcov) $G_{q_{v}}+E\left(U^{\prime}\right)\left[G_{q_{v} q_{v}}\left(d q_{v} / d \operatorname{cov}\right)+G_{q_{v} q_{k}}\left(d q_{k} / d \operatorname{cov}\right)+\right.$ $\left.\mathrm{G}_{\mathrm{q}_{\mathrm{v}} \mathrm{T}_{1}}\left(\mathrm{dT} \mathrm{T}_{1} / \mathrm{dcov}\right)\right]=\mathrm{T}_{1}(\mathrm{~d} \lambda / \mathrm{dcov})+\lambda\left(\mathrm{d} \mathrm{T}_{1} / \mathrm{dcov}\right)$

(VI.1)(ii) (dE(U')/dcov) $\left\{E\left[p_{k}(t)\right]+\operatorname{cov}\left[U^{\prime}, p_{k}(t)\right] E\left(U^{\prime}\right)-1-C^{\prime}(q(t))\right\}+$ $E\left(U^{\prime}\right)\left\{E\left(U^{\prime}\right)-1-\operatorname{cov}\left[U^{\prime}, p_{k}(t)\right]\left(d E\left(U^{\prime}\right) / d \operatorname{cov}\right) E\left(U^{\prime}\right)-2\right.$ $\left.C^{\prime \prime}(q(t))\left[\left(d q_{v} / d \operatorname{cov}\right)+\left(d q_{k} / d \operatorname{cov}\right)\right]\right\}=e^{\delta t}(d \lambda / d \operatorname{cov} v)$

(VI.1)(iii) $-T_{1}\left(d q_{v} / d \operatorname{cov}\right)-q_{v}\left(d T_{1} / d \operatorname{cov}\right)-d q_{k} / d \operatorname{cov}=0$

(VI.1)(iv)

$\left(d E\left(U^{\prime}\right) / d \operatorname{cov}\right) A+E\left(U^{\prime}\right)\left\{\left(d q_{v} / d \operatorname{cov}\right) N_{T_{1}}{ }_{0}{ }^{T}{ }^{T} e_{1} e^{-\delta t} d t+q_{v} N_{T_{1}}{ }^{\prime} e^{-\delta T_{1}}\left(d T_{1} / d \operatorname{cov}\right)-\right.$

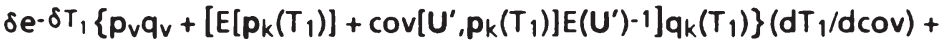

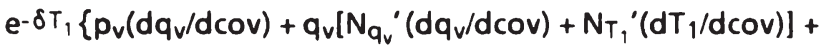
$\left[E\left[p_{k}\left(T_{1}\right)\right]+\operatorname{cov}\left[U^{\prime}, p_{k}\left(T_{1}\right)\right] E\left(U^{\prime}\right)^{-1}\right]\left(d q_{k} / d \operatorname{cov}\right)+q_{k}\left(T_{1}\right)\left[E\left(U^{\prime}\right)^{-1}-\right.$ $\left.\left.\left.\operatorname{cov}\left[U^{\prime}, p_{k}\left(T_{1}\right)\right]\left(d E\left(U^{\prime}\right) / d \operatorname{dcov}\right) E\left(U^{\prime}\right)-2\right]-C^{\prime \prime}\left(q\left(T_{1}\right)\right)\left(d q_{k} / d \operatorname{cov}+d q_{v} / d \operatorname{cov}\right)\right\}\right\}=$ $\lambda\left(d q_{k} / d \operatorname{cov}+d q_{v} / d \operatorname{cov}\right)+q\left(T_{1}\right)(d \lambda / d \operatorname{cov}) ;$

Es ist $\quad A={ }_{0} \int^{T}{ }_{1} q_{v} N_{T_{1}}{ }^{\prime} e^{-\delta t} d t+\left\{p_{v} q_{v}+\left\{E\left[p_{k}\left(T_{1}\right)\right]+\right.\right.$ $\left.\left.\operatorname{cov}\left[U^{\prime}, p_{k}\left(T_{1}\right)\right] E\left(U^{\prime}\right)-1\right\} q_{k}\left(T_{1}\right)-C^{\prime}\left(q\left(T_{1}\right)\right)\right\} e^{-\delta T_{1}}$ 
(VI.1)(v) $\quad d E\left(U^{\prime}\right) / d \operatorname{dcov}=$ $E\left\{U^{\prime \prime}\left\{\left[_{0} T^{\top}:\left[p_{v}+q_{v} N_{q_{v}}{ }^{\prime}-C^{\prime}(q(t))\right] e^{-\delta t} d t\right]\left(d q_{v} / d c o v\right)+\right.\right.$ $\left.\left.\left.\left[p_{k}(t)-C^{\prime}(q(t))\right]\right\} e^{-\delta t}\left(d q_{k} / d c o v\right)+\left(d T_{1} / d \operatorname{cov}\right)_{\eta} \int^{T_{1}} q_{v} N_{T_{1}}{ }^{\prime} e^{-\delta t} d t\right\}\right\}$.

In Matrizenform geschrieben wird das Gleichungssystem VI.1 zu VI.2.

(VI.2)

$\left[\begin{array}{ccccc}A_{1} & A_{2} & A_{3} & -T_{1} & A_{5} \\ B_{1} & B_{2} & 0 & -e \delta t & B_{5} \\ C_{1} & 0 & C_{3} & -q\left(T_{1}\right) & C_{5} \\ -T_{1} & -1 & -q_{v} & 0 & 0 \\ E_{1} & E_{2} & E_{3} & 0 & 1\end{array}\right]\left[\begin{array}{l}d q_{v} \\ d q_{k}(t) \\ d \lambda \\ d T_{1} \\ d E\left(U^{\prime}\right)\end{array}\right]=\left[\begin{array}{l}0 \\ -1 \\ -q_{k}\left(T_{1}\right) e^{\delta t} \\ 0 \\ 1\end{array}\right]\left[\begin{array}{l}d \operatorname{cov}] \\ -\end{array}\right]$

Dabei erfolgten die Setzungen:

$$
\begin{aligned}
& A_{1}=E\left(U^{\prime}\right)_{0} J^{\top}{ }_{1}\left[2 N_{q_{v}}{ }^{\prime}-C^{\prime \prime}(q(t))\right] e^{-\delta t} d t<0, \\
& A_{2}=-E\left(U^{\prime}\right) C^{\prime \prime}(q(t)) e^{-\delta t}<0, \\
& A_{3}=E\left(U^{\prime}\right)\left\{{ }_{0} S^{T}{ }_{1} N_{T_{1}}{ }^{\prime} e^{-\delta t} d t+\left[p_{v}+q_{v} N_{q_{v}}{ }^{\prime}-C^{\prime}(q(t))\right] e^{-\delta T} .\right\}>{ }_{<} 0, \\
& A_{5}={ }_{0} \int^{T} 1\left[p_{v}+q_{v} N_{q_{v}}{ }^{\prime}-C^{\prime}(q(t))\right] e^{-\delta t} d t>0 \text {, } \\
& B_{1}=B_{2}=-E\left(U^{\prime}\right) C^{\prime \prime}(q(t))<0 \text {, } \\
& C_{1}=E\left(U^{\prime}\right)\left\{{ }_{0} S^{T_{1}} N_{T_{1}}{ }^{\prime} e^{-\delta t} d t+\left[p_{v}+q_{v} N_{q_{v}}{ }^{\prime}-C^{\prime \prime}(q(t))\right] e^{-\delta T_{1}-\lambda}\right\}><0, \\
& C_{3}=E\left(U^{\prime}\right)\left\{2 q_{v} N_{T_{1}}{ }^{\prime}-\delta\left\{p_{v} q_{v}+\right.\right. \\
& \left.\left.\left[E\left[p_{k}\left(T_{1}\right)\right]+\operatorname{cov}\left[U^{\prime}, p_{k}\left(T_{1}\right)\right] E\left(U^{\prime}\right)^{-1}\right] q_{k}\left(T_{1}\right)\right\}\right\} e^{-\delta T_{1}}<0, \\
& C_{5}=A-\operatorname{cov}\left[U^{\prime}, p_{k}\left(T_{1}\right)\right] E\left(U^{\prime}\right)-1 e^{-\delta} T_{1}>0, \\
& E_{1}=-E\left(U^{\prime \prime}\right)_{0} \int^{T} 1_{1}\left[p_{v}+q_{v} N_{q_{v}}{ }^{\prime}-C^{\prime}(q(t))\right] e^{-\delta t} d t>0,
\end{aligned}
$$




$$
\begin{aligned}
& E_{2}=-E\left\{U^{\prime \prime}\left[p_{k}(t)-C^{\prime}(q(t))\right]\right\} e^{-\delta t}>0, \\
& E_{3}=-E\left(U^{\prime \prime}\right)_{0} j^{\top}{ }_{1} q_{v} N_{T_{1}}{ }^{\prime} e^{-\delta t} d t>0 .
\end{aligned}
$$

Das Gleıchungssystem liefert bereits für die Systemdeterminante kein eindeutiges Vorzeichen:

$$
\begin{aligned}
\Delta & =T_{1}\left[-T_{1}\left(B_{2} C_{3}-B_{5} C_{3} E_{2}-B_{2} C_{5} E_{3}\right)+e^{\delta t}\left(A_{2} C_{3}+A_{3} C_{5} E_{2}-A_{5} C_{3} E_{2}-\right.\right. \\
& \left.A_{2} C_{5} E_{3}\right)- \\
& \left.q\left(T_{1}\right)\left(A_{3} B_{5} E_{2}+A_{5} B_{2} E_{3}\right)\right]+T_{1}\left(B_{1} C_{3}+B_{5} C_{1} E_{3}-B_{5} C_{3} E_{1}-B_{1} C_{5} E_{3}\right)- \\
& e^{\delta t}\left(A_{1} C_{3}+A_{2} C_{5} E_{1}+A_{5} C_{1} E_{3}-A_{5} C_{3} E_{1}-A_{2} C_{1}-A_{1} C_{5} E_{3}\right)+ \\
& q\left(T_{1}\right)\left(A_{3} B_{5} E_{1}+A_{5} B_{1} E_{3}-A_{1} B_{5} E_{3}-A_{3} B_{1}\right)+ \\
& q_{v}\left[-T_{1}\left(B_{2} C_{5} E_{1}+B_{5} C_{1} E_{2}-B_{1} C_{5} E_{2}-B_{2} C_{1}\right)+\right. \\
& e^{\delta t}\left(A_{2} C_{5} E_{1}+A_{5} C_{1} E_{2}-A_{1} C_{5} E_{2}-A_{2} C_{1}\right)- \\
& \left.q\left(T_{1}\right)\left(A_{1} B_{2}+A_{2} B_{5} E_{1}+A_{5} B_{1} E_{2}-A_{5} B_{2} E_{1}-A_{2} B_{1}-A_{1} B_{5} E_{2}\right)\right]>=0 .
\end{aligned}
$$

Und bspw. für $\Delta_{1}$ :

$$
\begin{aligned}
& \Delta_{1}=\left(E_{3}-q_{v} E_{2}\right)\left[T_{1} C_{5}-q\left(T_{1}\right) A_{5}\right]+ \\
& q_{k}\left(T_{1}\right) e^{-\delta T_{1}}\left(E_{3}-E_{2}\right)\left(A_{5} e^{\delta t}-T_{1} B_{5}\right)+\left(A_{3}-q_{v} A_{2}\right) q\left(T_{1}\right)- \\
& q_{k}\left(T_{1}\right) e^{-\delta T_{1}}\left(A_{3}-A_{2}\right)-T_{1}\left[C_{3}+q_{k}\left(T_{1}\right) e^{\left.-\delta T_{1} B_{2} B_{5}\right]>_{<} 0 .} .\right.
\end{aligned}
$$

Da keine eindeutigen Ergebnisse erhalten werden können, wird die komparative Analyse hier nicht weiter fortgesetzt.

\section{Anhang VII}

(zu S.222)

Die Beweisführung für ein $B(t) \in(0,1)$ im Falle risikoaverser Handelspartner erfolgt mittels Widerspruch. Eine ähnliche Beweisführung im statischen Kontext eines Modells mit Produktionsunsicherheit findet man bei Sutinen (1975).

Es wird gezeigt, daß der $A$ bbauunternehmer weder ein $B(t)=0$, noch ein $B(t)=1$ wählt, wenn er die Indexierung des Reservenpreises so setzt, daß der Reservenbesitzer ein bestimmtes Erwartungsnutzenniveau $U_{R}{ }^{*}$ nicht 
unterschreitet. Mit $U_{R}{ }^{*}=U_{R}\left(G_{R}{ }^{*}\right)$ erhalt der Reservenbesitzer einen bestimmten Mindestgewınn. Für den Abbauunternehmer lautet die Problemstellung (vgl. 6.94):

(VII.1)

$$
\begin{aligned}
& \max E\left\{U_{A}\left[{ }_{0} \delta^{\top}(p(t)-c-[\alpha(t)+B(t) p(t)]) q(t) e^{-} \delta t d t\right]\right\} \\
& (\alpha(t), B(t)) \\
& \text { u.d.B. } E\left\{U_{R}\left[{ }_{0} \int^{\top}[\alpha(t)+B(t) p(t)] q(t) e^{-\delta} \delta t t\right]\right\} \geq U_{R}{ }^{*} \text {, } \\
& \alpha(t), \beta(t) \geq 0 \text {. }
\end{aligned}
$$

Dies führt für nichtnegative Werte $\alpha(t), B(t)$ zu den notwendigen Bedingungen

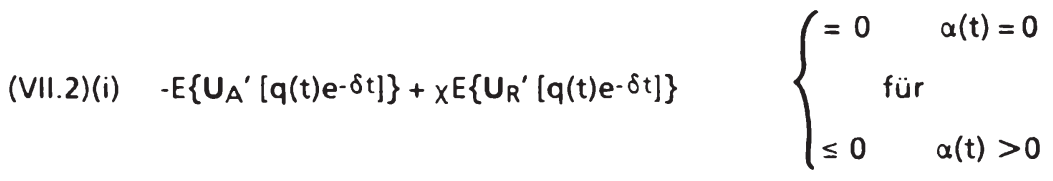

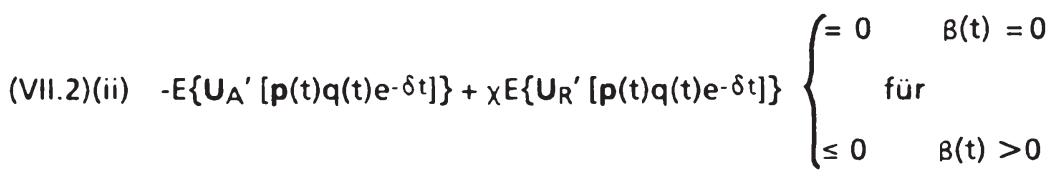

(VII.2)(iii) $E\left(U_{R}\left(G_{R}\right)\right)-U_{R}{ }^{*}$

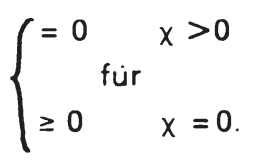

Aus (i) und (ii) folgt

$(V I I .3)(i)^{\circ} E\left(U_{A^{\prime}}\right)=x E\left(U_{R}{ }^{\prime}\right)$

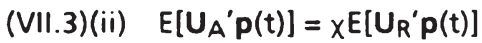

und weiter

(VII.4) $\quad E\left[U_{A}^{\prime} p(t)\right] E\left(U_{A^{\prime}}\right)^{-1}=E\left[U_{R}^{\prime} p(t)\right] E\left(U_{R}{ }^{\prime}\right)^{-1}$. 
Sei $B(t)=0$, d.h. der Reservenbesıtzer würde einen vom Rohstoffpreis unabhängigen Reservenpreis erhalten und der Abbauunternehmer das Marktrısiko in Form von Pressrisiko tragen.

Es würde dann gelten $E\left[U_{R}^{\prime} p(t)\right]=E\left(U_{R}^{\prime}\right) E[p(t)]$. Der Grenznutzen des Reservenbesitzers wäre unabhängig von den Realisationen der Zufallsvariablen $p(t)$.

In VII. 4 ergäbe dies $E\left[U_{A^{\prime}} p(t)\right]=E\left(U_{A^{\prime}}\right) E[p(t)]$. Für den risıkoaversen $A b b a u$ unternehmer gilt jedoch $E\left[U_{A^{\prime}} p(t)\right]=E\left(U_{A^{\prime}}\right) E[p(t)]+\operatorname{cov}\left[U_{A^{\prime}, p(t)}\right)$ mit $\operatorname{cov}\left[U_{A^{\prime}}, p(t)\right]<0$, soda $B\left[U_{A^{\prime}} p(t)\right]<E\left(U_{A^{\prime}}\right) E[p(t)]$ einen Widerspruch dazu bildet. Der risikoaverse Abbauunternehmer wählt $B(t)>0$.

Sei $B(t)=1$, d.h. der Reservenbesitzer würde einen vom Rohstoffpreis abhängigen Reservenpreis derart erhalten, daß er das gesamte Marktrisiko trüge: Es würde dann gelten $E\left[U_{A^{\prime}} p(t)\right]=E\left(U_{A^{\prime}}\right) E[p(t)]$, der Grenznutzen des Abbauunternehmers wäre unabhängig von den Realisationen der $\mathrm{Zu}$ fallsvariablen $p(t)$.

In VII.4 ergäbe dies $E\left[U_{R}^{\prime} p(t)\right]=E\left(U_{R}^{\prime}\right) E[p(t)]$. Jedoch gilt für den risikoaversen Reservenbesitzer $E\left[U_{R^{\prime}} p(t)\right]=E\left(U_{R^{\prime}}\right) E[p(t)]+\operatorname{cov}\left[U_{R^{\prime}}, p(t)\right]$, mit $\operatorname{cov}\left[U_{R^{\prime}}, p(t)\right]<0$, so daß auch hier ein Widerspruch abgeleitet werden kann zu

$$
E\left[U_{R}^{\prime} p(t)\right]<E\left(U_{R}{ }^{\prime}\right) E[p(t)] .
$$

Der risikoaverse Reservenbesitzer wird ein $B(t)=1$ nicht akzeptieren.

Die Widersprüche für $B(t)=0$ bzw. $B(t)=1$ lösen sich auf, wenn man im Falle $B(t)=0$ dem risikoaversen Reserven besitzer einen risikoneutralen Abbauunternehmer gegenüberstellt bzw. im Falle $B(t)=1$ dem risikoaversen Abbauunternehmer einen risikoneutralen Reservenbesitzer gegenüberstellt.

Der Parameter $\alpha(t)$ nimmt dabei positive oder negative Werte an:

$\beta(t)=0 \Rightarrow \alpha(t) \geq 0$, denn für $\beta(t)=0$ ist $G_{R}{ }^{*}={ }_{0} \int^{\top} \alpha(t) q(t) e^{-\delta t} d t$ und aus $G_{R}(t)=\alpha(t) q(t) e^{-\delta t}$ folgt $\alpha(t)=G_{R}(t) q(t)-1 e \delta t \geq 0$, für $G_{R}(t) \geq 0$.

$B(t)=1 \Rightarrow \alpha(t) \leq 0$, denn für $\beta(t)=1$ ist $G_{A}{ }^{*}={ }_{0} \int^{T}-q(t)[c+\alpha(t)] q(t) e^{-\delta t} d t$ und aus $G_{A}(t)=-q(t)[c+\alpha(t)] q(t) e^{-\delta t}$ folgt $a(t)=-\left[c+G_{A}(t) q(t)-1 e^{\delta t}\right]<0$, für $G_{A}(t) \geq 0$. 


\section{Anhang VIII}

(zu S.226)

Um die auf Seite 227 gemachte Behauptung abzuleiten, werden für einen gegebenen $A b b a u p f a d\{q(t)\}$ und ein gegebenes Erwartungsnutzenniveau $E\left\{U_{A}\right\}=U_{A}$ * die Substitutionsraten zwischen den Parametern $\alpha(t)$ und $B(t)$ bestimmt.1)

Aus der Zielfunktion folgt unter diesen Annahmen:

(VIII.1) $\quad(d \alpha / d B)_{A^{*}}=-E\left[U_{A^{\prime}} p(t)\right] E\left(U_{A^{\prime}}\right)^{-1}<0$.

Mit dem Terminus des Risikobias $\Gamma_{\mathrm{A} 2}$ kann VIII. 1 umgeschrieben werden zu (VIII.1') $(d \alpha / d B) U_{A^{*}}=-N(q(t)) \Gamma_{A 2}{ }^{-1}$.

Weiter ist es notwendig, zu wissen, ob die Substitution entlang einer konkaven oder konvexen Indifferenzkurve erfolgt.

(VIII.2) $\quad\left(d^{2} \alpha / d B^{2}\right) U_{A^{*}}=E\left\{U_{A^{\prime \prime}}\left[p(t)-N(q(t)) \Gamma_{A 2^{-1}}\right]^{2} q(t) e^{-\delta t}\right\} E\left(U_{A^{\prime}}\right)^{-1}<0$.

In Schaubild VIII. 1 ist die Indifferenzkurve eines Abbauunternehmers in Abhängigkeit der Parameter $\alpha(t)$ und $B(t)$ dargestellt.

Je kleiner für ein gegebenes $\alpha(t)$ der Anteil $B(t)$ ist, desto größer ist das Erwartungsnutzenniveau des Abbauunternehmers.

1) Zu dieser Vorgehensweise vgl. Sutinen (1980), S.174f. 
Schaubild VIII.1: Indifferenzkurve eines Abbauunternehmers

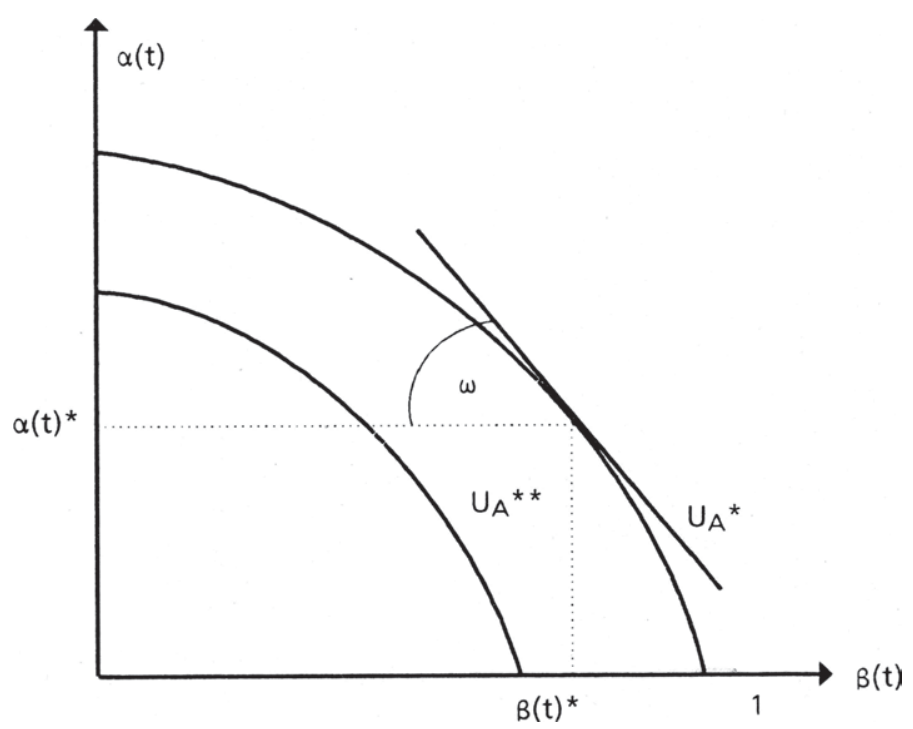

Bei einem gegebenen Erwartungsnutzenniveau $U_{A}$ * zeigt der Abbauunternehmer die Bereitschaft, für sinkende Anteile $B(t)$ mit abnehmender Rate steigende $\alpha(t)$ hinzunehmen .

Es gilt $\tan \omega=-N(q(t)) \Gamma_{A 2}-1$, und wegen VIII.2 folgt die Relation

$$
(d \alpha / d \beta)_{\alpha>0, B=0}>(d \alpha / d B)_{\alpha^{*}, \beta^{*}} \Leftrightarrow \Gamma_{A 1}>\Gamma_{A 2} .
$$

Der Risikobias eines risikoaversen Abbauunternehmers nimmt mit steigendem $B(t)$ entlang seiner Indifferenzkurve $a b$.

Ausgehend von der Zielfunktion eines Reservenbesitzers in 6.84 gelangt man zu den Ergebnissen

(VIII.3) $\quad(d \alpha / d \beta) U_{R^{*}}=-E\left[U_{R}^{\prime} p(t)\right] E\left(U_{R}^{\prime}\right)-1<0$

$\left(V I I I .3^{\prime}\right) \quad(d \alpha / d \beta) U_{R}^{*}=-N(q(t)) \Gamma_{R}-1$ 
(VIII.4) $\quad\left(d^{2} \alpha / d \beta^{2}\right) U_{R^{*}}=-E\left\{U_{R}{ }^{\prime \prime}\left[p(t)-N(q(t)) \Gamma_{R}{ }^{-1}\right]^{2} q(t) e^{-\delta t}\right\} E\left(U_{R}{ }^{\prime}\right)-1>0$.

Die Darstellung der Indifferenzkurve eines Reservenbesitzers erfolgt in Schaubild VIII. 2.

Schaubild VIII.2: Indifferenzkurve eines Reservenbesitzers

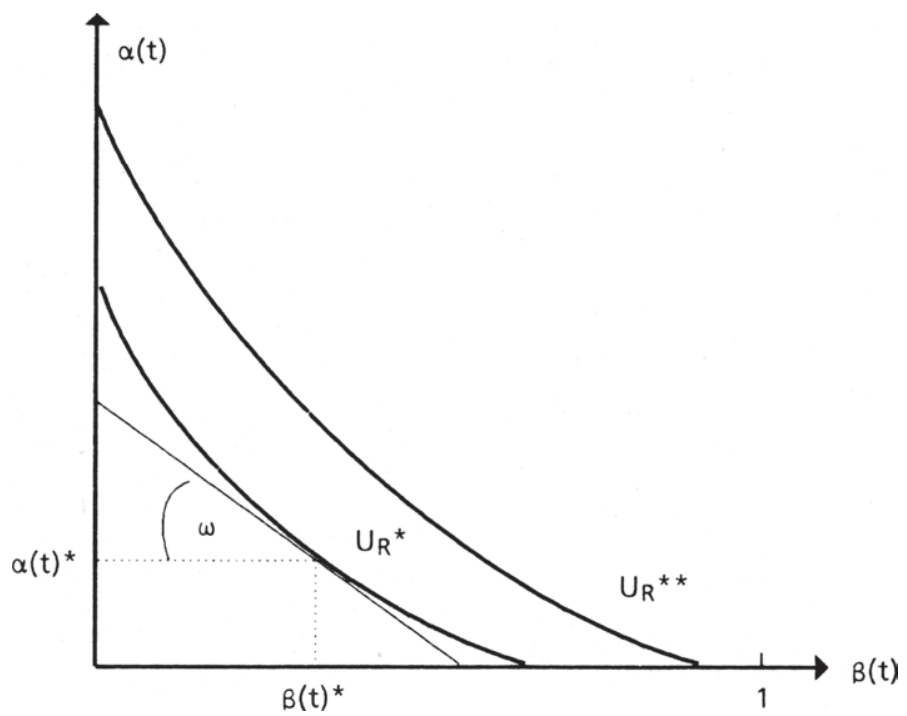

Je größer für ein gegebenes $\alpha(t)$ der Anteil $B(t)$ ist, desto größer ist das Erwartungsnutzenniveau des Reservenbesitzers.

Für ein gegebenes Erwartungsnutzenniveau $U_{R}{ }^{*}$ ist der Reservenbesitzer bereit, für sinkende Anteıle $B(t)$ mit zunehmender Rate steigende $\alpha(t)$ hinzunehmen.

Hier ist $\tan \omega=-\mathrm{N}(\mathrm{q}(\mathrm{t})) \Gamma_{\mathrm{R}}-1$, und mit VIII.3, VIII.4 folgt

$$
(d \alpha / d \beta)_{\alpha>0, \beta=0}<(d \alpha / d B)_{\alpha^{*}, B^{*}} \Leftrightarrow \Gamma_{R 1}<\Gamma_{R 2}
$$


Es bezeichnet $\Gamma_{R 1}$ den Risikobias bei den Parameterkombinationen $\alpha(t)>0$ und $B(t)=0$. $\Gamma_{R 2}$ den Risikobias bei den Kombinationen $\alpha(t), B(t)>0$.

Der Risikobias eines risikoaversen Reservenbesitzers nimmt mit sinkendem $B(t)$ entlang seiner Indifferenzkurve $a b$.

Schaubild VIII.3 stellt die Indifferenzkurven der Handelspartner eınander gegenüber und zeigt die Verhandlungspositionen auf.1)

Schaubild VIII.3: Kontraktkurve

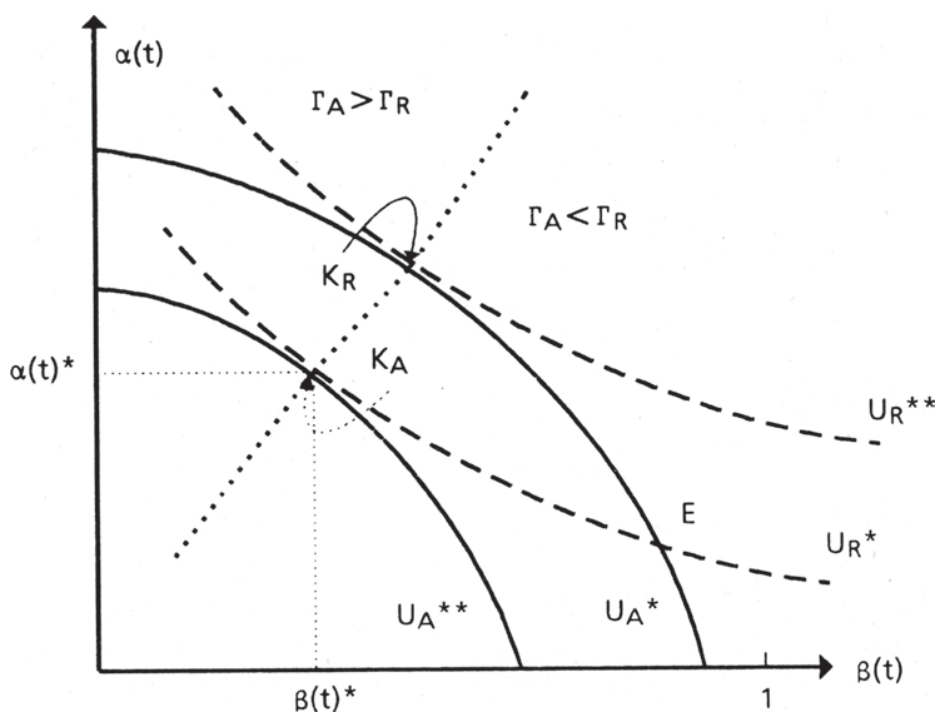

1) Vgl. Sutinen (1980), S. 175 zu diesem Schaubild. 
Sind beide Handelspartner risikoavers, dann wird eine Verteilung der Parameter entlang der durch $K_{A} K_{R}$ gehenden Kontraktkurve gewahlt werden. Von der Ausgangssituation $E$ aus (erstes Verhandlungsangebot) lassen sich Parameterkombinationen $K_{A}$ und $K_{R}$ erreichen, bei denen sich mindestens einer der Handelspartner besser stellt.

Der Optimierungsansatz 6.94 würde zu der Kombination $\left(\alpha(t)^{\star}, B(t)^{\star}\right)$ führen und ließe dem Abbauunternehmen den gesamten, aus der Verhandlungssituation resultierenden Nutzengewinn zufallen. In $K_{R}$ würde der Nutzengewinn dem Reservenbesitzer zufallen.

Entlang der Kontraktkurve gilt die Gleichheit der Substitutionsraten zwischen $\alpha(t)$ und $B(t)$, bzw. die Gleichheit der Risikobiases $\Gamma_{A 2}$ und $\Gamma_{R}$ :

$$
(d \alpha / d B)_{U_{A}^{*}}=(d \alpha / d B) U_{R^{*}} \Leftrightarrow \Gamma_{A 2}=\Gamma_{R} .
$$

Oberhalb (unterhalb) der Kontraktkurve liegen Parameterkombinationen für die der Risikobias des Abbauunternehmers größer (kleiner) als der Risikobias des Reservenbesitzers ist.

Wenn einer der beiden Handelspartner risikoneutral und der andere risikoavers ist, werden die folgenden Parameterkombinationen erhalten:

(1) risikoaverser Reservenbesitzer / risikoneutraler Abbauunternehmer, d.h. $\Gamma_{R}>1, \Gamma_{A 2}=1 \Rightarrow(d \alpha / d B) U_{R^{*}}=-N(q(t)) \Gamma_{R^{-1}}>-N(q(t))=(d \alpha / d B) U_{A^{*}} \Rightarrow B(t)$ $=0$, wegen VIII. 4 .

(2) risikoneutraler Reservenbesitzer/ risikoaverser Abbauunternehmer, d.h. $\Gamma_{R}=1, \Gamma_{A 2}>1 \Rightarrow(d \alpha / d B) U_{R^{*}}=-N(q(t))<-N(q(t)) \Gamma_{A 2^{-1}}=(d \alpha / d B) U_{A^{*}} \Rightarrow B(t)$ $=1$, wegen VIII. 2 .

Beide Fälle werden in Schaubild VIII.4 dargestellt.

Um zu einem paretoeffizienten "Tausch" der Parameter $\alpha(t)$ und $\beta(t) z u$ kommen, bzw. sich diesem anzunähern, wird von dem Marginalkalkül die Angleichung der Substıtutionsraten bzw. der Risikobiases verlangt. Da für 
Schaubild VIII.4: Parameterkombinationen für unterschiedliches Risıkoverhalten

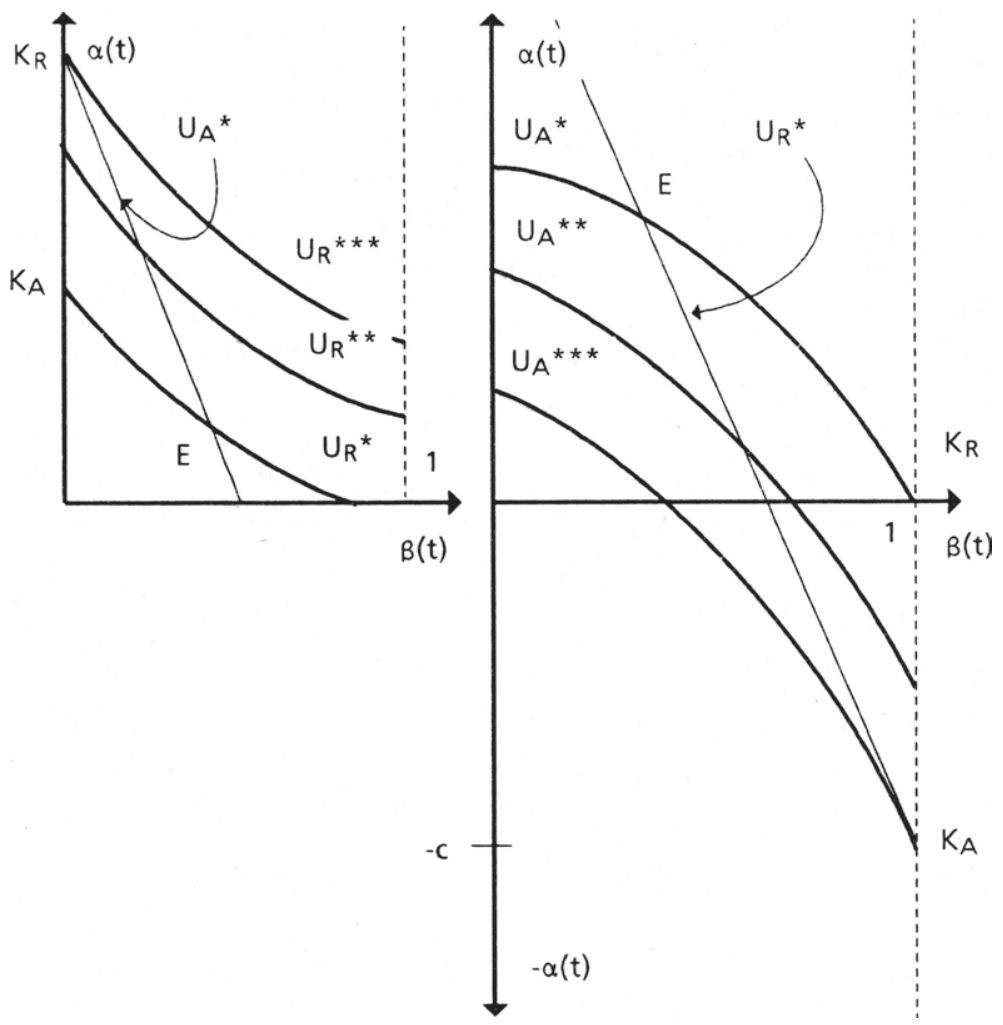

das risikoneutrale Wirtschaftssubjekt die Substitutionsrate unabhängıg von den Werten der Parameter $\alpha(t), B(t)$ ist, paßt sich das risikoaverse Wirtschaftssubjekt mit seiner Substitutionsrate (seinem Risikobias) an: Der Risikobias eines risikoaversen Reservenbesitzers (Abbauunternehmers) geht gegen eins, wenn $B(t)$ gegen null (eins) geht. $\alpha(t)$ nimmt dabel seinen größten (kleinsten) Wert an.

Aus der notwendigen Bedingung des Abbauunternehmers in Verbindung mit der Nachfrage auf dem Rohstoffmarkt (Gleichung 6.90) erhält man für 
ein gegebenes Erwartungsnutzenniveau eine Beziehung zwischen $\alpha(t)$ und $B(t)$ :

$$
\alpha(t)=(1-B(t)) N(q(t)) \Gamma_{A 2^{-1}}-c .
$$

Hieraus folgt für die beiden Fälle:

(i) $\Gamma_{R}>1, \Gamma_{A 2}=1$ und $B(t)=0$ ergibt $\alpha(t)=N(q(t))-c$,

(ii) $\Gamma_{R}=1, \Gamma_{A 2}>1$ und $B(t)=1$ ergibt $\alpha(t)=-c$.

Im ersten Fall würde ein $\alpha(t)$ gewählt werden, das einer mengenbezogenen Förderabgabe entspräche (Gleichung 6.72 für $\Gamma_{\mathrm{A} 2}=1$ ).

Im zweiten Fall fordert das Abbauunternehmen für die Abgabe des gesamten Erlöses aus der Vermarktung des Rohstoffes von dem Reservenbesitzer die Übernahme der Abbaukosten. 


\section{Literaturverzeichnis}

Anderson, R. C. (1977),

Tax Policy and Secondary Material Use, Journal of Environmental Economics and Management, Vol. 4, S. 68 -82.

Arrow, K. J. (1970),

Essays in the Theory of Risk Bearing, Amsterdam.

Banks, F. E. (1980),

Prices and Price Formation in the Market for Non-Fuel Minerals, in: Siebert, H. (Hrsg.), Erschöpfbare Ressourcen, Schriften des Vereins für Socialpolitik, N.F. Bd. 108, Berlin, S. $475-491$.

Barnett, H. J. (1979),

Scarcity and Growth Revisited, in: Smith, V. K. (Hrsg.), Scarcity and Growth Reconsidered, Baltimore and London, S. 163 -217.

Barnett, H. J. / Morse, Ch. (1963),

Scarcity and Growth. The Economics of Natural Resource

Availability, Baltimore.

Barrows (1980),

World Petroleum Arrangements, New York.

Baumol, W. J. (1959),

Business Behavior, Value and Growth, New York.

Birchenhall, C. / Grout, P. (1980),

Market Structure and Price Controls with Exhaustible Resources, in: Siebert, H. (Hrsg.), Erschöpfbare Ressourcen, Schriften des Vereins für Socialpolitik, N.F. Bd. 108, Berlin, S. $423-433$. 
Blair, R. D. (1974),

Random Input Prices and the Theory of the Firm, Economic Inquiry, Vol. 12, S. $214-226$.

Blair, R. D. / Heggestad, A. A. (1977),

Price Discrimination under Uncertainty, in: Blinder, A. S. I

Friedman, Ph. (Hrsg.), Natural Resources, Uncertainty, and

General Equilibrium Systems, New York, S. 53 -65.

Blattner, N. (1976),

Wettbewerbspolitische Implikationen neuerer Ansätze in der Theorie der Firma, in: Bombach, G./ Gahlen, B. (Hrsg.), Probleme der Wettbewerbstheorie und -politik, Tübingen, S. 227-297.

Blinder, A. S. / Friedman, Ph. (Hrsg.) (1977),

Natural Resources, Uncertainty, and General Equilibrium Systems, New York.

Bobrow, D. B. / Kudrle, R. T. (1976),

Theory, Policy, and Resource Cartels. The Case of OPEC, Journal of Conflict Resolution, Vol. 20, S. 3 -56.

Bosson, R. / Varon, B. (1977),

The Mining Industry and the Developing Countries, New York.

Brown, G. M. / Field, B. (1979),

The Adequacy of Measures for Signalling the Scarcity of Natural Resources, in: Smith, V. K. (Hrsg.), Scarcity and Growth Reconsidered, Baltimore and London, S. 218 -248.

Brown, K. C. (1969),

Bidding for Offshore Oil: Toward an Optimal Strategy, Dallas.

Burchard, H.-J. (1980),

Funktionsweise der internationalen Ölmärkte, in: Siebert, $\mathrm{H}$. (Hrsg ), Erschöpfbare Ressourcen, Schriften des Vereins für Socialpolitik, N.F. Bd. 108, Berlin, S. $461-474$.

Burness, S. H. (1976),

On the Taxation of Nonreplenishible Natural Resources, Journal of Environmemtal Economics and Management, Vol. 3, S. 289 311. 
Butler, D. S. L. (1979),

Commodity Options, in: Keyser, E. de (Hrsg.), Guide to World Commodity Markets, London and New York 1979, S. 29-35.

Cigno, A. (1979),

Depletion of Natural Resources and Accumulation of Capital when Population is Endogenous. An Activity Analysis, Metroeconomica, Vol. 31, S. 33 -54.

Common, M. / Pearce, D. W. (1973),

Adaptive Mechanisms, Growth and the Environment: The Case of Natural Resources, Canadian Journal of Economis, Vol. 6 , S. $289-300$.

Cremer, J. / Weitzman, M. L. (1976), OPEC and the Monopoly Price of World OIl, European Economic Review, Vol. 8, S. 155 -164.

Crommelin, M. / Thompson, A. R. (Hrsg.) (1976) Mineral Leasing as an Instrument of Public Policy, Vancouver.

Cropper, M. C. / Weinstein, M. C. / Zeckhauser, R. J. (1978), The Optimal Consumption of Depletable Natural Resources: An Elaboration, Correction, and Extension, Quarterly Journal of Economics, Vol. 92, S. $337-344$.

Dam, K. W. (1965),

Oil and Gas Licensing and the North Sea Oil, Journal of Law and Economics, Vol. 8, S. 11 -44.

Dam, K. W. (1970),

The Pricing of Noth Sea Gas in Britain, Journal of Law and Economics, Vol. 13, S. 51 - 75.

Dam, K. W. (1974),

The Evolution of North Sea Licensing Policy in Britain and Norway, Journal of Law and Economics, Vol. 17, S. $213-263$

Dam, K. W. (1976),

Oil Resources, Who Gets What How?, Chicago and London. 
Dasgupta, P. S. (1974),

Some Theoretical Explorations in the Economics of Exhaustible Resources, in: Gottınger, H. W. (Hrsg.), Systems Approaches and Environmental Problems, International Symposıum: Schloß Reısensburg, Gottıngen, S. $193-214$.

Dasgupta, P. S. (1981),

Resource Pricing and Technological Innovations under Oligopoly: A Theoretical Exploration, Scandinavian Journal of Economics, Vol. 83, S. $289-317$.

Dasgupta, P. S. / Eastwood, R. / Heal, G. (1978), Resource Management in a Trading Economy, Quarterly Journal of Economics, Vol. 92, S. 297 -306.

Dasgupta, P. S. / Gilbert, R. J. / Stiglitz J. E. (1980), Invention and Innovation under Alternative Market Structures: The Case of Natural Resources, Econometric Research Program, Research Memorandum No. 263, Prınceton, New Jersey.

Dasgupta, P. S. / Heal, G. (1974),

The Optimal Depletion of Exhaustible Resources, Review of Economic Studies, Symposium on the Economis of Exhaustible Resources, Vol. 41, S. 3 - 28.

Dasgupta, P. S. / Heal, G. (1979), Economic Theory and Exhaustible Resources, Cambridge.

Dasgupta, P. S. / Mitra, T. (1980),

On Some Problems in the Formulation of Optimum Policies When Resources are Depletable, Working Paper No. 226, State University New York at Stoney Brook and Cornell University. Dasgupta, P. S. / Stiglitz J. E. (1980a), Market Structure and Resource Depletion. A Contribution to the Theory of Intertemporal Monopolistic Competition, Econometric Research Program, Research Memorandum No. 261, Princeton, New Jersey. 
Dasgupta, P. S. / Stiglitz J. E. (1980b),

Market Structure and Resource Extraction under Uncertainty, Econometric Research Program, Research Memorandum No. 262, Princeton, New Jersey.

Dasgupta, P. S. / Stıglitz J. E. (1981),

Resource Depletion under Technological Uncertainty, Econometrica, Vol. 49, S. $85-104$.

Davison, R. (1978),

Optimal Depletion of an Exhaustible Resource with Research and Development towards an Alternative Technology, Review of Economic Studies, Vol. 45, S. 355 -367.

Deshmukh, S. D. / Pliska, S. R. (1980),

Optimal Consumption and Exploration of Renewable Resources under Uncertainty, Econometrica, Vol. 48, S. $177-200$.

Devarajan, S. / Fisher, A. C. (1981), Hotelling's Economics of Exhaustible Resources: Fifty Years Later, Journal of Economic Literature, Vol. 19, S. 65 -73.

Doo-Soon Ahn (1981),

Gemeinschaftsunternehmungen in Entwicklungsländern, Tübingen.

Eswaran, M. / Lewis, T. R. (1980),

A Note on Market Structure and the Search for Exhaustible Resources, Resources Paper No. 55, University of British Columbia.

Eswaran, M. / Lewis, T. R. (1981),

Production from Differentiated Exhaustible Resources in Oligopolistic Markets, Resources Paper No. 63.

Fischer, D. / Gately, D. / Kyle, J. F. (1975),

The Prospects for OPEC: A Critical Survey of Models of the World Oil Market, Journal of Development Economics, Vol. 2, S. 363 386. 
Fritzsche, M. (1979),

Fiskalregıme von Bergbauvorhaben, Frankfurt am Main.

Fritzsche, M. (1981),

Fiskalregime, in: Schanze, E., u.a. (1981), Rohstofferschließungsvorhaben in Entwicklungslàndern, Teıl 2: Probleme der Vertragsgestaltung, Frankfurt am Main, S. $118-163$.

Fisher, A. C. (1979),

Measures of Natural Resource Scarcity, in: Smıth, V. K. (Hrsg.), Scarcity and Growth Reconsidered, Baltımore and London, S. 249 -275 .

Garnaut, R. / Ross, A. C. (1975),

Uncertainty, Risk Aversion and the Taxing of Natural Resource Projects, Economic Journal, Vol. 85, S. 272 -287.

Gately, D. (1979),

OPEC Pricing and Output Decisions. A Partition Function Approach to OPEC Stability, in: Brams, S. J. / Schotter, A. / Schwödiauer, G. (Hrsg.), Applied Game Theory, Würzburg und Wien, S. $303-312$.

Gately, D. / Kyle, J. / Fisher, D. (1976),

Strategies for OPEC Pricing Decisions, European Economic Review, Vol. 10, S. $209-230$.

Gehrels, F. (1980),

Die intertemporale Allokation natürlicher Ressourcen in offenen Volkswirtschaften, in: Siebert, H. (Hrsg.), Erschöpfbare Ressourcen, Schriften des Vereins für Socialpolitik, N.F. Bd. 108, Berlin, S. $597-612$.

Gilbert, R. J. (1977),

Resource Extraction with Differential Information. American Economic Review, Papers and Proceedings, Vol. 67, S. 250 -254.

Gilbert, R. J. (1979a),

Optımal Depletion of an Uncertain Stock, Review of Economic Studies, Vol. 46, S. 47-57. 
Gilbert, R. J. (1979b),

Search Strategies and Private Incentives for Resource Exploration, in: Pindyck, R. S. (Hrsg.), Advances in the Economics of Energy and Resources, Vol. 2, Greenwich, Conn., S. 149 -169.

Gilbert, R. J. / Goldman, S. (1978),

Potential Competition and the Monopoly Price of an Exhaustible Resource, Journal of Economic Theory, Vol. 17, S. 319 -331.

Gilley, O. W. / Karels, G. V. (1981),

The Competitive Effect and Bonus Bidding: New Evidence, Bell Journal of Economics, Vol. 12, S. $637-648$.

Gordon, R. L. (1967),

A Reinterpretation of the Pure Theory of Exhaustion, Journal of Political Economy, Vol. 75, S. 274-286.

Gordon, R. L. (1983),

Resource Procurement: A Case Study of US Electric Utilities, in:

Pearce, D. W. / Siebert, H. / Walter, I. (Hrsg.), Risk and the Political Economy of Resource Development, London, S. 408 -436.

Gottwald, D. / Güth, W. (1980),

Allocation of Exhaustible Resources in Oligopolisitic Markets. A

Dynamic Game Approach, Energy Economics, Vol. 2, S. 208 -222.

Grathwohl, M. (1978),

Energieversorgung, Berlin und New York.

Gray, L. C. (1914),

Rent under the Assumption of Exhaustibility, Journal of Political Economy, Vol. 28, S. 466 -489.

Grossman, S. J. (1975),

Rational Expectations and the Econometric Modeling of Markets Subject to Uncertainty: A Baysian Approach, Journal of Econometrics, Vol. 3, S. $255-272$.

Grossman, S. J. / Stiglitz, J. E. (1976), Information and Competitive Price Systems, American Economic Review, Papers and Proceedings, Vol. 66, S. 246 -253. 
Hanson, D. A. (1977a),

The Allocation of a Natural Resource when the Cost of a Substitute is Uncertain, Economic and Social Measurement, Vol. 6, S. $189-201$.

Hanson, D. A. (1977b),

Competitive Price Behavior of an Exhaustible Resource Where the Rate of Substitution is Constrained, International Economic Review, Vol. 18, S. $135-149$.

Hanson, D. A. (1978),

Efficient Transitions from a Resource to a Substitute Technology in an Open Economic Growth Context, Journal of Economic Theory, Vol. 17, S. $99-113$.

Hartwick, J. M. (1978),

Exploitation of Many Deposits of an Exhaustible Resource, Econometrica, Vol. 46, S. $201-217$.

Heal, G. (1975),

Economic Aspects of Natural Resource Depletion, in: Pearce, D. W. / Rose, J. (Hrsg.), The Economics of Natural Resource Depletion, London, S. $118-139$.

Heal, G. (1977),

Guarantees and Risk-Sharing, Review of Economic Studies, Vol. 44, S. $549-560$.

Heal, G. (1979),

Uncertainty and the Optimal Supply Policy for an Exhaustible Resource, in: Pindyck, R. S. (Hrsg.), Advances in the Economics of Energy and Resources, Vol. 2, Greenwich, Conn., S. $119-147$.

Heal, G. (1980),

Intertemporal Allocation and Intergenerational Equity, in: Siebert, H. (Hrsg.), Erschöpfbare Ressourcen, Schriften des Vereins für Socialpolitik, N.F. Bd. 108, Berlin, S. $37-73$.

Heal, G. (1981),

Scarcity, Efficiency and Disequilibrium in Resource Markets, Scandinavian Journal of Economics, Vol. 83, S. 334- -351. 
Hillman, A. L. / Long, N. v. (1982),

Pricing and Depletion of an Exhaustible Resource when there is Anticipation of Trade Disruption, Quarterly Journal of Economics, Vol. 98, S. $215-233$.

Hnyilicza, E. / Pindyck, R. S. (1976),

Pricing Policy for a Two Part Exhaustible Resource Cartel: The

Case of OPEC, European Economic Review, Vol. 8, S. 139 -154.

Hoel, M. (1978a),

Resource Extraction when a Future Substitute has an Uncertain Cost, Review of Economic Studies, Vol. 45, S. 637 -644.

Hoel, M. (1978b),

Resource Extraction under Some Alternative Market Structures, Meisenheim am Glan.

Hoel, M. (1978c),

Resource Extraction, Uncertainty and Learning, Bell Journal of Economics, Vol. 9, S. 642 -645.

Hoel, M. (1980),

Extraction of an Exhaustible Resource under Uncertainty, Königstein /Ts.

Hoel, M. (1981),

Resource Extraction by a Monopolist with Influence over the Rate of Return on Non Resource Assets, International Economic Review, Vol. 22, S. 147 -157.

Holt, C. A. (1980),

Competitive Bidding for Contracts under Alternative Auctions

Procedures, Journal of Political Economy, Vol. 88, S. 433 -445.

Hotelling, H. (1931),

The Economics of Exhaustible Resources, Journal of Political Economy, Vol. 39, S. $137-175$.

Houthakker, H. S. (1959),

The Scope and Limits of Futures Trading, in: Abramovitz, M., The Allocation of Economic Resources, Stanford.

Howe, C. W. (1979),

Natural Resource Economics, New York, u.a. 
Ingham, A. / Simmons, P. (1975),

Natural Resources and Growing Population, Review of Economic Studies, Vol. 41, S. $191-206$.

Jaeger, K. (1980),

Ansatze zu eıner ökonomischen Theorie des Recycling, in: Siebert, H. (Hrsg.), Erschöpfbare Ressourcen, Schriften des Vereins für Socialpolitik, N.F. Bd. 108, Berlin, S. 149 -174.

Jevons, W. S. (1865),

The Coal Question, London.

Kalt, J. P. (1981),

The Economics and Politics of an Oil Price Regulation, Cambridge.

Kalt, J. P. / Otten, A. L. (1981),

The Theory of Nonrenewable Resource, Extraction under Discontinous Price Policy, MIT-Working Paper No. MIT-EL 81-026 WP.

Kamien, M. I. / Schwartz, N. L. (1977a),

A Note on Resource Usage and Market Structure, Journal of Economic Theory, Vol. 15, S. 394 -397.

Kamien, M. I. / Schwartz, N. L. (1977b),

Disaggregated Intertemporal Modells with an Exhaustible Resource and Technical Advance, Journal of Environmemtal Economics and Management, Vol. 4, S. $271-288$.

Kamien, M. I. / Schwartz, N. L. (1978),

Optimal Exhaustible Resource Depletion with Endogenous Technical Change, Review of Economic Studies, Vol. 45, S. 179 -196.

Kemp, M. C. (1976),

How to Eat a Cake of Unknown Size, in: Kemp, M. C., Three Topics in the Theory of International Trade, Amsterdam, u.a., S. 297 -308 .

Kemp, M. C. / Long, N.v. (1980a) (Hrsg.)

Exhaustible Resources, Optimality and Trade, Amsterdam, New York, Oxford. 
Kemp, M. C. / Long, N.v. (1980b),

On the Optimal Order of Exploitation of Deposits of an Exhaustible Resource: The Case of Certainty, in: Siebert, H. (Hrsg.), Erschöpfbare Ressourcen, Schriften des Vereins für Socialpolitik, N.F. Bd. 108, Berlin, S. $301-317$.

Kemp, M. C. / Long, N.v. (1980c),

Resource Ectraction under Conditions of Common Access, in:

Kemp, M. C. / Long, N.v. (Hrsg.), Exhaustible Resources, Optimality and Trade, Amsterdam, New York, Oxford, S. 127 -135.

Kemp, M. C. / Long, N.v. (1982),

The Efficiency of Competitive Markets in a Context of Exhaustible Resources, in: Eichhorn, W. (Hrsg.), The Economics of Natural Resources, Wien, u.a., S, $205-211$.

Kemp, M. C. / Okoguchi, K. (1979),

Optimal Policies for Exhaustible Resources in Open Economies, Zeitschrift für die gesamte Staatswissenschaft, Bd. 135, S. 207 215.

Keyser, E. de, u.a. (Hrsg.) (1979),

Guide to World Commodity Markets, London und New York.

Khalatbari, F. (1977),

Market Imperfections and the Optimum Rate of Depletion of

Natural Resources, Economica, Vol.44, S. $409-414$.

Krelle, W. (1976),

Preistheorie. Teil 1: Monopol-und Oligopoltheorie, Tübingen.

Kydland, F. / Prescott, E. (1977);

Rules Rather than Discretion: The Inconsistency of Optimal Plans, Journal of Political Economy, Vol. 85, S. 473 -491.

Labys, W. C. (1980),

Market Stucture, Bargaining Power and Resource Price Formation, Lexington, Mass.

Lecomber, R. (1979),

The Economics of Natural Resources, London. 
Lee, D. R. (1979),

Price Controls on Non-Renewable Resources: An Intertemporal Analysis, Southern Economic Journal, Vol. 75, S. 274 -286.

Leiter, R. D. (1976),

An Introduction to Resources, in:Leiter, R. D. / Friedlander, S. L.

(Hrsg.), Economics of Resources, New York, S. 9-14.

Leiter, R. D. / Friedlander, S. L. (1976) (Hrsg.),

Economics of Resources, New York.

Leland, H. E. (1978),

Optimal Risk Sharing and the Leasing of Natural Resources, with Application to Oil and Gas Leasing on the OCS, Quarterly Journal of Economics, Vol. 92, S. 413-437.

Levhari, D. / Liviatan, N. (1977),

Notes on Hotelling's Economics of Exhaustible Resources, Canadian Journal of Economics, Vol. 10, S. 177 -192.

Levhari, D. / Pindyck, R. S. (1981),

The Pricing of Durable Exhaustible Resources, Quarterly Journal of Economics, Vol. 96, S. $365-377$.

Levy, H. / Sarnat, M. (1975),

The World Oil Crisis: A Portfolio Interpretation, Economic Inquiry, Vol. 13, S. $361-372$.

Lewis, T. R. (1976),

Monopoly Exploitation of an Exhaustible Resource, Journal of

Environmemtal Economics and Management, Vol. 3, S. 198 -204.

Lewis, T. R. (1977),

Attitudes towards Risk and the Optimal Exploitation of an Exhaustible Resource, Journal of Environmemtal Economics and Management, Vol. 4, S. $111-119$.

Lewis, T. R. / Schmalensee, R. (1980a),

On Oligopolistic Markets for Nonrenewable Natural Resources, Quarterly Journal of Economics, Vol. 95, S. 475 -491. 
Lewis, T. R. / Schmalensee, R. (1980b),

Cartel and Oligopoly Pricing of Nonreplenishable Natural Resources, in: Liu, P. T. (Hrsg.), Dynamic Optimization and Mathematical Economics, New York and London, S. 133-156.

Liu, P. T. (Hrsg.) (1980),

Dynamic Optimization and Mathematical Economics, New York and London.

Long, N. v. (1974),

International Borrowing for Resource Extraction, International

Economic Review, Vol. 15, S. 168 -183.

Long, N. v. (1975),

Resource Extraction under the Uncertainty about Possible Nationalization, Journal of Economic Theory, Vol. 10, S. 42 -53.

Long, N. v. (1983),

Risk and Resource Economics: The State of the Art, in: Pearce, D. W. / Siebert, H. / Walter, I. (Hrsg.), Risk and the Political Economy of Resource Development, London, S. 71 -91.

Loury, G. C. (1978),

The Optimal Exploitation of an Unknown Reserve, Review of

Economic Studies, Vol. 45, S. $621-636$.

Loury, G. C. (1980),

A Theory of Oligopoly: Cournot Equilibrium in Exhaustible Resource Markets with Fixed Supplies, Mimeographed.

Lusky, R. (1975),

Optimal Taxation Policies for Conservation and Recycling, Journal of Economic Theory, Vol. 11, S. 315 -328.

Malthus, Th. R. (1798),

First Essay on Population, London.

Markovitz, H. M. (1959),

Portfolio Selection - Efficient Diversification of Investment, New York.

Mill, J. S. (1848),

Principles of Political Economy with Some of Their Applications to Social Philosophy, London. 
Mitra, T. (1978),

Efficient Growth with Exhaustible Resources in a Neoclassical Model, Journal of Economic Theory, Vol. 17, S. $114-129$.

Mohnfeld, J. (1982),

Die Weltolındustrie im Strukturwandel, Wirtschaftsdienst, Bd. 62 , S $184-190$.

Nordhaus, W. D. (1973),

The Allocation of Energy Resources, Brookıngs Papers on Economic Activity, Vol. 3, S. 529-576.

Pearce, D. W. / Rose, J. (Hrsg.) (1975),

The Economics of Natural Resource Depletion, London.

Pearce, D. W. / Siebert, H. / Walter, I. (Hrsg.), Risk and the Political Economy of Resource Development, London.

Peck, A. E. (1975), Hedging and Income Stability: Concepts, Implications, and an Example, American Journal of Agricultural Economics, Vol. 57, S. $410-419$.

Peck, A. E. (1976),

Futures Markets, Supply Response, and Price Stability, Quarterly Journal of Economics, Vol. 90, S. 407 -423.

Peterson, F. M. / Fisher, A. C. (1977),

The Exploitation of Extractive Resources, Economic Journal, Vol. 87, S. $681-721$.

Pethig, R. (1980),

Intertemporale Allokation mit erschöpfbaren Ressourcen und endogenen Innovationen, in: Siebert, H. (Hrsg.), Erschöpfbare Ressourcen, Schriften des Vereins für Socialpolıtik, N.F. Bd. 108, Berlin, S. 277 -295. 
Pflug, G. / Winckler, G. (1980),

Stabilitäts- und Ungleichgewichtsprobleme von Märkten für erschöpfbare Ressourcen, in: Siebert, H. (Hrsg.), Erschopfbare Ressourcen, Schriften des Vereins für Socıalpolitik, N.F Bd. 108, Berlin, S. $407-421$.

Pflug, G. / Winckler, G. (1982),

Speculation and Stability on Markets for Exhaustible Resources, in: Eichhorn, W. (Hrsg.), The Economics of Natural Resources, Würzburg und Wien, S. $205-211$.

Pindyck, R. S. (1978a), Gains to Producers from the Cartelization of Exhaustible Resources, Review of Economics and Statistics, Vol. 60, S. 238 -251.

Pindyck, R. S. (1978b),

The Optimal Exploration and Production of Nonrenewable Resources, Journal of Political Economy, Vol. 86, S. 841 -861.

Pindyck, R. S. (1979) (Hrsg.), Advances in the Economics of Energy and Resources, Greenwich, Conn.

Pindyck, R. S. (1980), Uncertainty and Exhaustible Resource Markets, Journal of Political Economy, Vol. 88, S. $1203-1225$.

Pindyck, R. S. (1981a), The Optimal Production of an Exhaustible Resource when Price is Endogenous and Stochastic, Scandinavian Journal of Economics, Vol. 83, S. $277-288$.

Pindyck, R. S. (1981b), Jointly Produced Exhaustible Resources, MIT Working Paper No. MIT-EL 81-003 WP.

Pratt, J.W. (1964), Risk Aversion in the Small and in the Large, Econometrica, Vol. 32, S. $122-136$.

Radetzki, M. (1978),

Market Structure and Bargaining Power. A Study of Three International Mineral Markets, Resources Policy, Vol. 4, S. 115 -125. 
Reece, D. K. (1978),

Competitive Bidding for Offshore Petroleum Leases, Bell Journal of Economics, Vol. 9, S. $369-384$.

Reece, D. K. (1979),

An Analysis of Alternative Bidding Systems for Offshore Oil, Bell Journal of Economics, Vol. 10, S. 659 -669.

Ricardo, D. (1817),

On the Principles of Political Economy and Taxatıon, London.

Roberts, B. (1980),

The Effects of Supply Contracts on the Output and Price of an

Exhaustıble Resource, Quarterly Journal of Economics, Vol. 95, 5. $245-260$.

Robson, A. J. (1979),

Sequentiell Exploitation of an Uncertain Deposit of a Depletable Natural Resource, Journal of Economic Theory, Vol. 21, S. 88 110.

Rothschild, M. / Stiglitz, J. E. (1970), Increasing Risk. A Definition, Journal of Economic Theory, Vol. 2, S. $225-243$.

Salant, S. W. (1976),

Exhaustible Resources and Industrial Structure. A Nash Cournot Approach to the World Oil Market, Journal of Political Economy, Vol. 84, S. $1079-1093$

Salant, S. W. (1982),

Imperfect Competition in the World Oil Market, Lexington, Mass.

Sandmo, A. (1971),

On the Theory of the Competitive Firm under Price Uncertainty, American Economic Review, Vol. 61, 5. 65 -73. 
Sauter-Servaes, F. (1980),

Der Übergang von einer erschöpfbaren Ressource zu einem synthetischen Substitut, in: Siebert, H. (Hrsg.), Erschöpfbare Ressourcen, Schriften des Vereins für Socialpolitik, N.F. Bd. 108, Berlin, S. $245-258$.

Schanze, E., u.a. (Hrsg.) (1981),

Rohstofferschließungsvorhaben in Entwicklungsländern, Teil 2:

Probleme der Vertragsgestaltung, Frankfurt.

Schanze, E. (1981),

Rechtsformenverwendung und gesellschaftsrechtliche Interessenvermittlung, in: Schanze, E., u.a. (Hrsg.) (1981), Rohstofferschließungsvorhaben in Entwicklungsländern, Teil 2: Probleme der Vertragsgestaltung, Frankfurt, S. $19-68$.

Schneider, H. K. / Schulz, W. (1983),

The Importing Country's Perspective: A Case Study of LNG, in:

Pearce, D. W. / Siebert, H. / Walter, I. (Hrsg.), Risk and the Political Economy of Resource Development, London, S. 374 -394.

Schmalensee, R. (1976),

Resource Exploitation Theory and the Behavior of the Oil Cartel, European Economic Review, Vol. 7, S. 257 -279.

Siebert, H. (Hrsg.) (1980),

Erschöpfbare Ressourcen, Schriften des Vereins für Socialpolitik, N.F. Bd. 108, Berlin.

Siebert, H. (Hrsg.) (1981),

Ökonomische Theorie natürlicher Ressourcen. Ein Überblick, Zeitschrift für Wirtschafts- und Sozialwissenschaften, Bd. 101, S. $267-298$.

Siebert, H. (Hrsg.) (1982a),

Resource Extraction with Closing Costs of a Mine, Discussion

Paper 204/82, Universität Mannheim.

Siebert, H. (Hrsg.) (1982b),

A Resource-Extraction Firm with Set-up Costs, Discussion Paper 226/82, Universität Mannheim.

Siebert, H. (Hrsg.) (1983),

Okonomische Theorıe natürlicher Ressourcen, Tübıngen. 
Siebert, H. (Hrsg.) (1984),

Economics of the Resource-Exporting Countries, Intertemporal Theory of Resource Supply and Trade, Greenwich, Conn.

Sinn, H. W. (1980a),

Besteuerung, Wachstum und Ressourcenabbau, in: Siebert, $\mathrm{H}$. (Hrsg.), Erschopfbare Ressourcen, Schriften des Vereins für Socialpolitik, N.F. Bd. 108, Berlin, S. $499-528$.

Sinn, H. W. (1980b),

Ökonomische Entscheidungen unter Ungewißheit, Tübingen. Sinn, H. W. (1983),

On the Optimal Taxation of Natural Resources, in: Pearce, D. W.I Siebert, H. / Walter, I. (Hrsg.), Risk and the Political Economy of Resource Development, London, S. 244-255.

Smiley, A. K. (1979),

Competitive Bidding under Uncertainty: The Case of Offshore Oil, Cambridge, Mass.

Smith, B. (1978),

Long Term Contracts for the Supply of Raw Materials in: Crawford, J. / Okita, S. (Hrsg.), Raw Materials and Pacific Economic Integration, London, S. 229-270.

Smith, B. (1979),

Security and Stability in Mineral Markets - The Role of Long-Term Contracts, World Economy, Vol. 2, S. $65-78$.

Smith, D. N. / Wells, L. T. (1975),

Negotiating Third-World Mineral Agreements, Cambridge, Mass.

Smith, J. L. (1980),

Bidding Behavior for Offshore Petroleum Leases, MIT Working Paper No. MIT-EL 80-006 WP.

Smith, J. L. (1982),

Risk Aversion and Bidding Behavior for Offshore Petroleum Leases, Journal of Industrial Economics, Vol. 30, S. 251 -269. 
Smith, V.K. (1978),

Measuring Natural Resource Scarcity: Theory and Practise, Journal of Environmemtal Economics and Management, Vol. 5, S. 150 $-171$.

Smith, V. K. (Hrsg.) (1979),

Scarcity and Growth Reconsidered, Baltimore and London.

Smith, V. K. / Krutilla, J. V. (1979),

The Economics of Natural Resource Scarcity: An Interpretive Introduction, in: Smith, V. K. (Hrsg.), Scarcity and Growth Reconsidered, Baltimore and London, S. 1 -35.

Smith, V. L. (1968),

Economics of Production from Natural Resources, American Economic Review, Vol. 58, S. 409 -431.

Smith, V. L. (1974),

An Optimistic Theory of Exhaustible Resources, Journal of Economic Theory, Vol. 9, S. 384 -396.

Smith, V. L. (1976),

Control Theory Applied to Natural and Environmental Resources. An Exposition, Journal of Environmental Economics and Management, Vol. 4, S. 1 -24.

Solow, R. M. (1974),

The Economics of Resources or the Resources of Economics, American Economic Review, Vol. 64, S. 1 -14.

Solow, R. M. (1977),

Monopoly, Uncertainty, and Exploration, in: Blinder, A. S. / Friedman, Ph. (Hrsg.), Natural Resources, Uncertainty, and General Equilibrium Systems, New York, S. 17-34.

Stiglitz, J. E. (1979),

A Neoclassical Analysis of the Economics of Natural Resources, in: Smith, V. K. (Hrsg.), Scarcity and Growth Reconsidered, Baltimore and London, S. $36-66$.

Stollery, K. R. (1981a),

Mineral Depletion with Cost at the Extraction Limit: A Model Applied to the Behavior of Prices in the Nickel Industry, Waterloo Economic Series No. 118, Waterloo. 
Stollery, K. R. (1981b),

Price Controls on Nonrenewable Resources when Capacity is Constrained, Waterloo Economic Series No. 119, Waterloo.

Streit, M. E. (1979a),

Terminkontraktmärkte und ihr möglicher Lösungsbeitrag zu internationalen Rohstoffproblemen, Discussion Paper 119/79, Universität Mannheim.

Streit, M. E. (1979b),

Zur Funktionsweise von Terminkontraktmärkten, Discussion Paper 139/79, Universität Mannheim.

Streit, M. E. / Quick, R. (1981),

Informationsaktivitäten und Preisbildungsprozesse auf Terminkontraktmärkten, Discussion Paper 170/81, Universität Mannheim.

Strotz, R. H. (1955),

Myopic and Inconsistency in Dynamic Utility Maximation, Review of Economic Studies, Vol. 23, S. 165 -180.

Sutinen, J.G. (1975),

The Rational Choice of Share Leasing and Implications for Efficiency, American Journal of Agricultural Economics, Vol. 57, S. $613-621$.

Sutinen, J .G. (1980),

Royalties and the Extraction Rate of an Exhaustible Resource, in: Liu, P. T. (Hrsg.), Dynamic Optimization and Mathematical Economics, New York and London, S. $169-178$.

Suzuki, H. / Ogawa, M. (1979),

International Trade with Exhaustible Natural Resources, Zeitschrift für Nationalökonomie, Bd. 39, S. 131 -142.

Sweeney, J. L. (1977),

Economics of Depletable Resources, Market Forces and Intertemporal Bias, Review of Economic Studies, Vol. 44, S. 125 -142. 
Telser, L.G. (1981),

Why There are Organized Future Markets, Law and Economics, Vol. 24, S. $1-22$.

Telser, L. G. / Higginbotham, H. N. (1977),

Organized Futures Markets: Costs and Benefits, Journal of Political Economy, Vol. 85, S. $969-1000$.

Tietenberg, T. (1980),

Substitution Bias in a Depletable Resource Model with Administered Prices, in: Siebert, H. (Hrsg.), Erschöpfbare Ressourcen, Schriften des Vereins für Socialpolitik, N.F. Bd. 108, Berlin, S. 529 $-552$.

Tobin, J. (1958),

Liquidity Preference as Behavior towards Risk, Review of Economic Studies, Vol. 25, S. 65 -86.

Uhler, R. S. (1981),

Oil Reserve Prices, Resources Paper No. 66.

Ulph, A. / Folie, G. (1980),

Exhaustible Resources and Cartels: An Intertemporal Nash-

Cournot Model, Canadian Journal of Economics, Vol. 13, S. 645 658.

Verleger, P. K. (1982),

The Determination of Official OPEC Crude Prices, Review of Economics and Statistics, Vol. 64, S. $177-183$.

Vousden, N. (1974), International Trade and Exhaustible Resources: A Theoretical Model, International Economic Review, Vol. 15, S. 149 -167.

Weinstein, M. C. / Zeckhauser, R. J. (1974),

Use Patterns for Depletable and Recycleable Resources, Review of Economic Studies, Symposium on the Economis of Exhaustible Resources, Vol. 41, S. $67-88$. 
Weınstein, M. C. / Zeckhauser, R. J. (1975),

The Optimal Consumption of Depletable Natural Resources, Quarterly Journal of Economics, Vol. 89, S. 371 -392.

Weizsäcker, C. C. v. (1980),

Leistet der Markt die optimale intertemporale Allokation der Ressourcen?, in: Siebert, H. (Hrsg.), Erschöpfbare Ressourcen, Schriften des Vereins für Socialpolitik, N.F. Bd. 108, Berlin, S.795814.

Williamson, O. E. (1971),

Managerial Discretion, Organization Form, and the Multi-division Hypothesis, in: Marris, R. / Wood, A. (Hrsg.), The Corporate Economy, Growth, Consumption, and Innovative Potential, London and Basingstoke, S. $343-386$.

Williamson, O. E. (1972),

Managerial Discretion Models, in: Rowley, C. K. (Hrsg.), Readings in Industrial Economics, Vol. 1, Theoretical Foundations, London and Basingstoke, S. $67-90$.

Wilson, R. (1977),

A Bidding Model of Perfect Competition, Review of Economic Studies, Vol. 44, S. $511-518$.

Working, H. (1970),

Economic Functions of Futures Markets, in: Bakken, H. H. (Hrsg.), Futures Trading in Livestock, Madison, S. 3-47.

Yuchtman, E. / Seashore, S. (1967),

A System Resource Approach to Organizational Effectiveness, American Sociological Review, Vol. 32, S. 891 -903. 


\section{STAATLICHE ALLOKATIONSPOLITIK IM MARKTWIRTSCHAFTLICHEN SYSTEM}

Band 1 Horst Siebert (Hrsg.): Umweltallokation im Raum. 1982.

Band 2 Horst Siebert (Hrsg.): Global Environmental Resources. The Ozone Problem. 1982.

Band 3 Hans-Joachim Schulz: Steuerwirkungen in einem dynamischen Unternehmensmodell. Ein Beitrag zur Dynamisierung der Steuerüberwälzungsanalyse. 1981.

Band 4 Eberhard Wille (Hrsg.): Beiträge zur gesamtwirtschaftlichen Allokation. Allokationsprobleme im intermediären Bereich zwischen öffentlichem und privatem Wirtschaftssektor. 1983.

Band 5 Heinz König (Hrsg.): Ausbildung und Arbeitsmarkt. 1983.

Band 6 Horst Siebert (Hrsg.): Reaktionen auf Energiepreissteigerungen. 1982.

Band 7 Eberhard Wille (Hrsg.): Konzeptionelle Probleme öffentlicher Planung. 1983.

Band 8 Ingeborg Kiesewetter-Wrana: Exporterlösinstabilität. Kritische Analyse eines entwicklungspolitischen Problems. 1982.

Band 9 Ferdinand Dudenhöffer: Mehrheitswahl-Entscheidungen über Umweltnutzungen. Eine Untersuchung von Gleichgewichtszuständen in einem mikroökonomischen Marktund Abstimmungsmodell. 1983.

Band 10 Horst Siebert (Hrsg.): Intertemporale Allokation. 1984.

Band 11 Helmut Meder: Die intertemporale Allokation erschöpfbarer Naturressourcen bei feh. lenden Zukunftsmärkten und institutionalisierten Marktsubstituten. 1984. 
Siebert, Horst (Hrsg.)

\section{UMWELTALLOKATION IM RAUM}

Frankfurt/M., Bern, 1982. V, 232 S.

Staatliche Allokationspolitik im marktwirtschaftlichen System. Bd. 1

ISBN 3-8204-5997-9

br. sFr. 45.-

In diesem Sammelband werden Beiträge zusammengefasst, die sich mit der Nutzung der Umwelt in ihren verschiedenen Funktionen (öffentliches Konsumgut, Rezeptor von Schadstoffen) unter dem besonderen Aspekt der räumlichen Dimensionen von Umweltgütern befassen. Der Band enthält wirtschaftspolitisch und theoretisch ausgerichtete Beiträge. Insbesondere wird versucht, die Bestimmung der anzustrebenden Umweltqualität in räumlichen Systemen (Regionen) und die für die Erreichung dieser Umweltqualität einzusetzenden Instrumente zu erklären.

Aus dem Inhalt: Wirkungsweisen umweltpolitischer Instrumente (Siebert) - Umwelt- und Regionalpolitik in der Bundesrepublik Deutschland (Vogt) - Bestimmung des anzustrebenden Umweltqualitätswertes (Dehez) - Allokation öffentlicher Güter bei Konsumentenmobilität (Dudenhöffer/Gebauer) - Mehrheitswahl als Instrument regionalisierter Umweltpolitik (Dudenhöffer) Intertemporale regionale Umweltallokation (Gebauer).

Siebert, Horst (Hrsg.)

\section{REAKTIONEN AUF ENERGIEPREISSTEIGERUNGEN}

Frankfurt/M., Bern, 1982.138 S.

Staatliche Allokationspolitik im marktwirtschaftlichen System. Bd. 6

ISBN 3-8204-7254-1

br. sFr. 31.-

Dieser Sammelband fasst vier Beiträge zur Anpassung der Industrienationen auf Energieverknappungen und Energiepreiserhöhungen zusammen. Die Beiträge gehen von den Preissteigerungen für Erdol 1973/74 und 1979/80 aus und untersuchen, welche Inzidenz diese Preissteigerungen auf makrookonomische Variable wie Preisniveaustabilität, Zahlungsbilanzsituation, Beschaftigung und Sektorstruktur haben und welche Anpassungen in den Industrienationen erfolgt sind oder vorgenommen werden sollten.

Aus dem Inhalt: Die Anpassung der Volkswirtschaft beim Ubergang zu neuen Energieversorgungssystemen: Die theoretische Grundlage eines Energiemodells für die Bundesrepublik Deutschland (Conrad) - Importieren wir Stagflation über steigende Rohstoffpreise? Keynesianische und monetaristische Ansichten (Schmid) - Absatzsteuern, Olförderung und das Allmendeproblem (Sinn) - Energiepreisentwicklung und Konsumallokation privater Haushalte (Zimmermann).

\section{Verlag Peter Lang Bern - Frankfurt a.M. New York}

Auslielerung Verlag Peter Lang AG. Jupiterstr. 15,CH-3000 Bern 15 Telefon $(0041 / 31) 321122$. Telex verl ch 32420

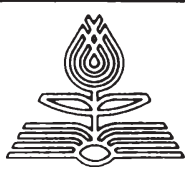

